\title{
Recognition Elements for Elongation Factor P on the Ribosome
}

\author{
Dissertation \\ for the award of the degree \\ "Doctor rerum naturalium" \\ of the Georg-August-Universität Göttingen
}

within the doctoral program: GGNB Biomolecules: Structure - Function - Dynamics of the Georg-August University School of Science (GAUSS)

submitted by

Jan Ole Frister

from Kiel, Germany

Göttingen, 2018 


\section{Thesis Committee}

Prof. Dr. Marina Rodnina

Department of Physical Biochemistry, Max Planck Institute for Biophysical Chemistry, Göttingen

Prof. Dr. Kai Tittmann

Department of Molecular Enzymology, Schwann-Schleiden Research Center, Georg August University, Göttingen

Prof. Dr. Holger Stark

Department of Structural Dynamics, Max Planck Institute for Biophysical Chemistry, Göttingen

\section{Members of the Examination Board}

Reviewer: Prof. Dr. Marina Rodnina

Department of Physical Biochemistry, Max Planck Institute for Biophysical Chemistry, Göttingen

Second Reviewer: Prof. Dr. Kai Tittmann

Department of Molecular Enzymology, Schwann-Schleiden Research Center, Georg August University, Göttingen

\section{Further members of the Examination Board}

Prof. Dr. Ralf Ficner

Department of Molecular Structural Biology, Institute for Microbiology and Genetics, Georg August University, Göttingen

Prof. Dr. Holger Stark

Department of Structural Dynamics, Max Planck Institute for Biophysical Chemistry, Göttingen

Prof. Dr. Henning Urlaub

Institute for Clinical Chemistry, University Medical Center, Göttingen

Prof. Dr. Wolfgang Wintermeyer

Research Group of Ribosome Dynamics, Max Planck Institute for Biophysical Chemistry, Göttingen

Date of the oral examination: 29.11.2018 


\section{Affidavit}

I hereby declare that my thesis „Recognition Elements for Elongation Factor P on the Ribosome " has been written independently and with no other sources and aids than quoted.

Jan Ole Frister

September the 30th, 2018

Göttingen, Germany 



\section{Publication}

Huter, P., Arenz, S., Bock, L.V., Graf, M., Frister, J.O., Heuer, A., Peil, L., Starosta, A.L., Wohlgemuth, I., Peske, F., Nováček, J., Berninghausen, O., Grubmüller, H., Tenson, T., Beckmann, R, Rodnina, M.V., Vaiana, A.C., Wilson, D.N. (2017). Structural Basis for Polyproline-Mediated Ribosome Stalling and Rescue by the Translation Elongation Factor EF-P. Mol Cell. 68, 515-527. 



\section{Table of Contents}

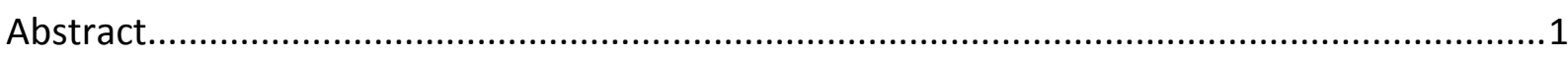

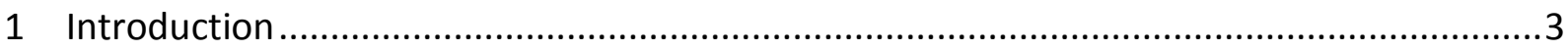

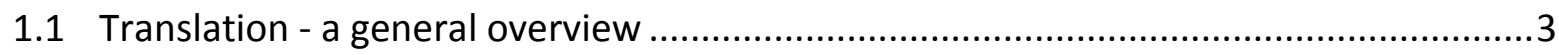

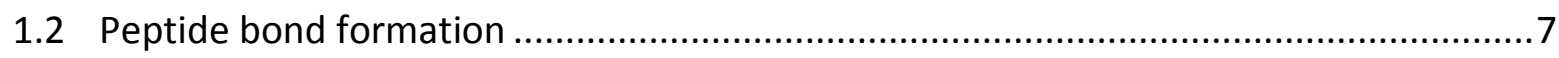

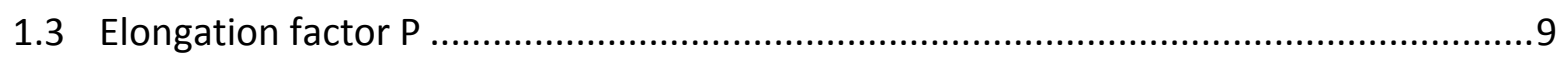

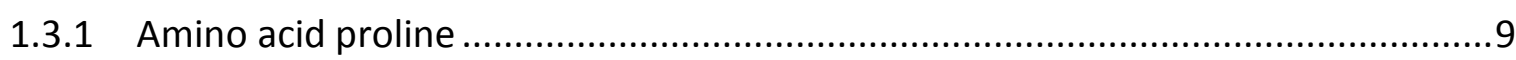

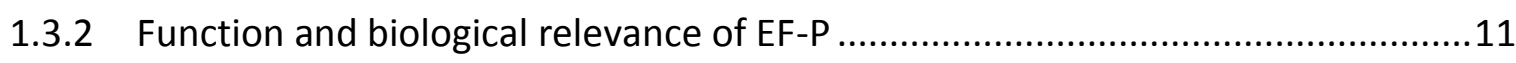

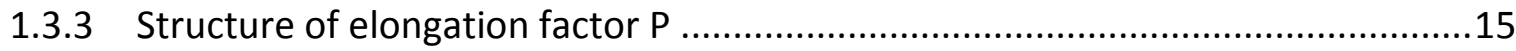

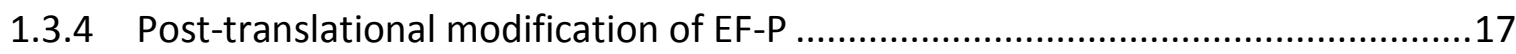

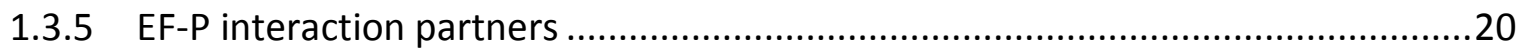

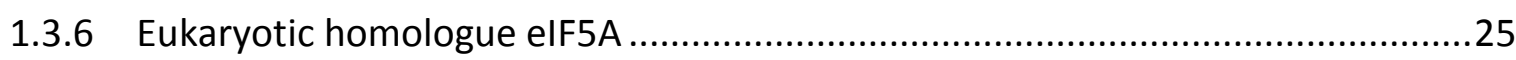

1.3.7 Post-translation modifications of e/alF5A ........................................................2

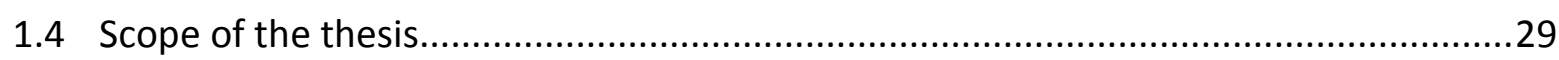

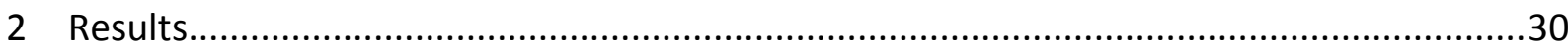

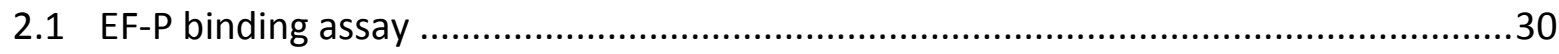

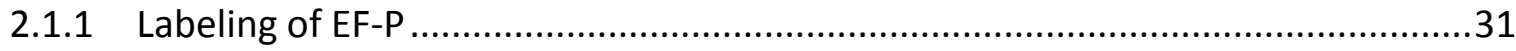

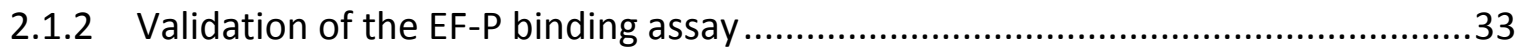

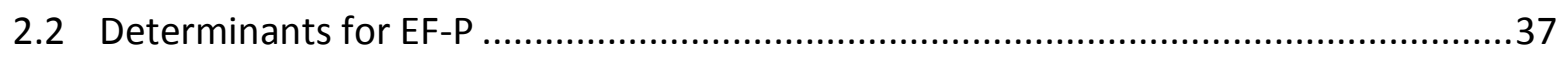

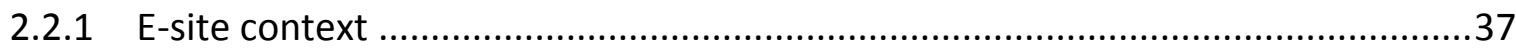

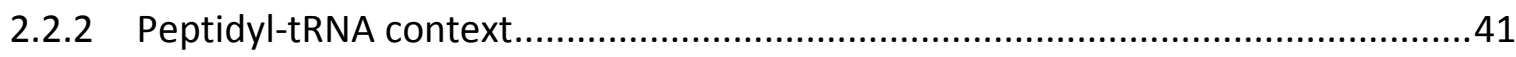

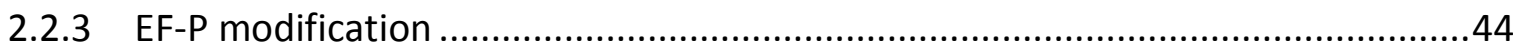

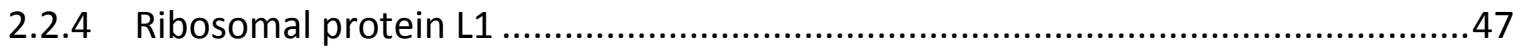

2.3 Catalysis of peptide bond formation by EF-P .........................................................

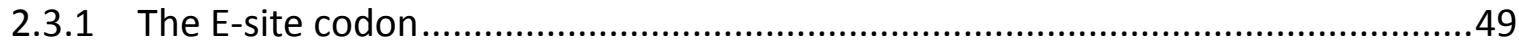

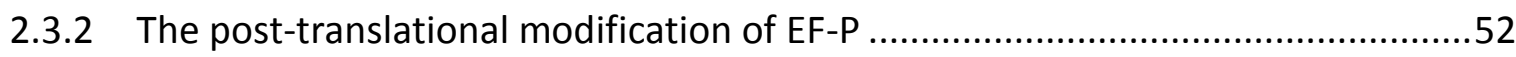

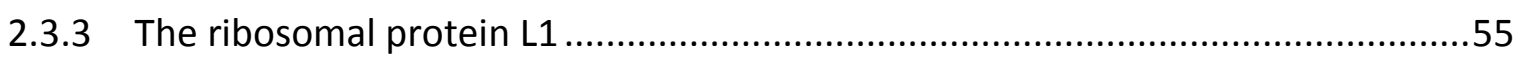

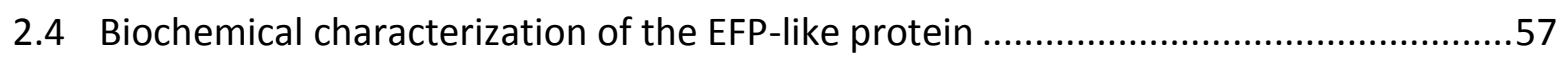

2.5 Mass-spectrometric quantification of EF-P in the cell .........................................60

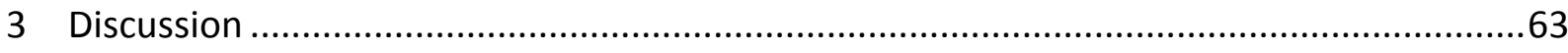

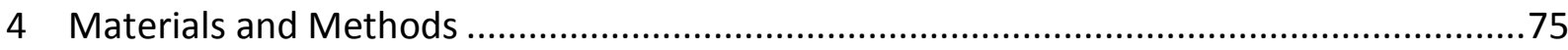

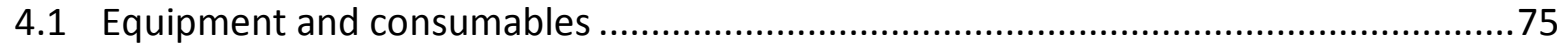

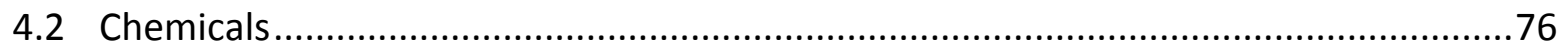

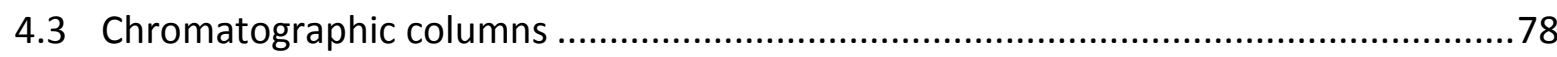




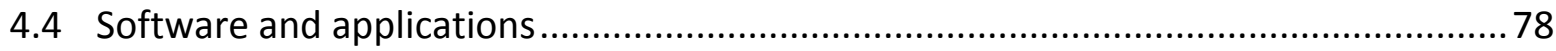

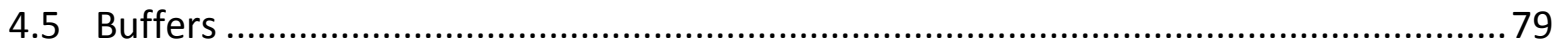

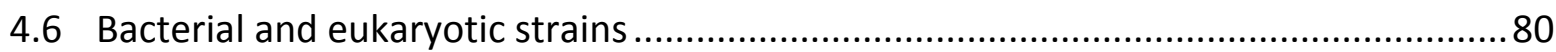

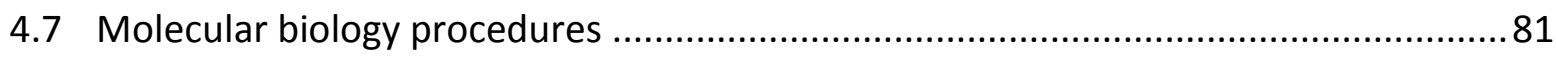

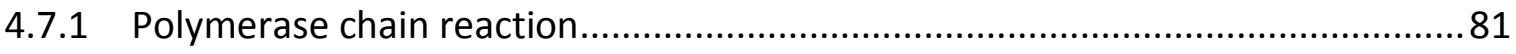

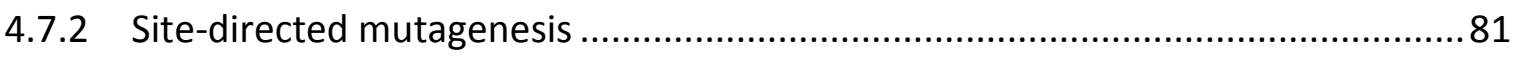

4.7.3 Site-directed mutagenesis using isothermal assembly .......................................82

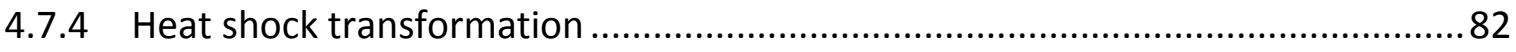

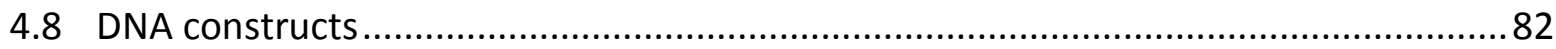

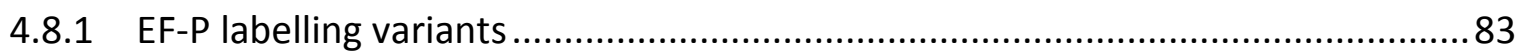

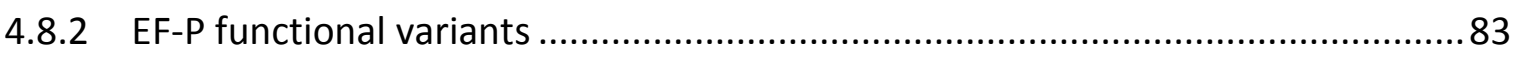

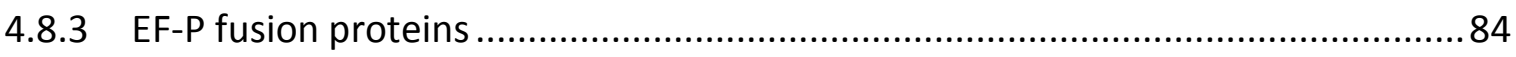

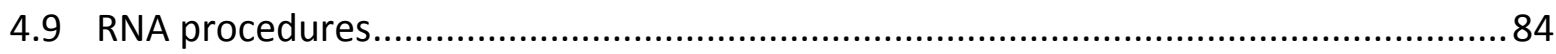

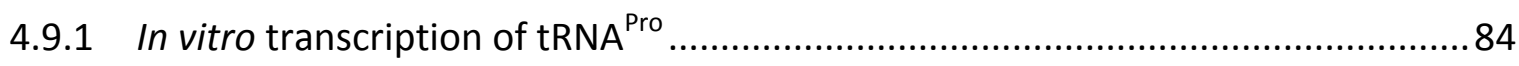

4.9.2 Denaturing polyacrylamide gel electrophoresis............................................. 85

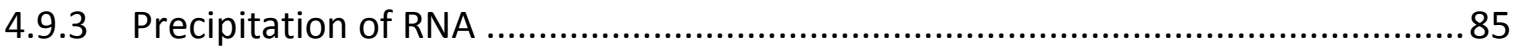

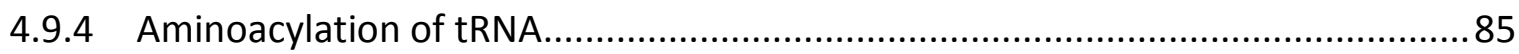

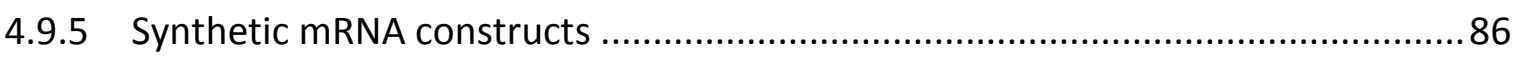

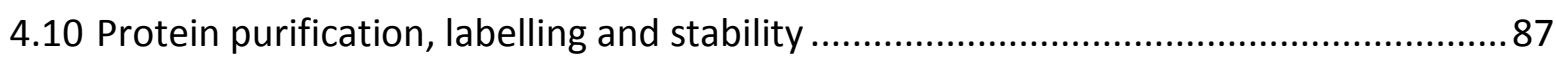

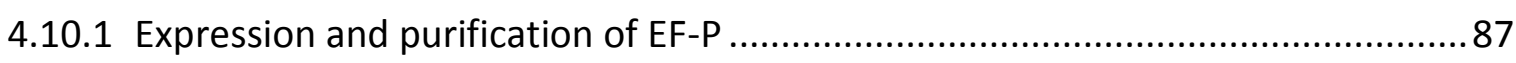

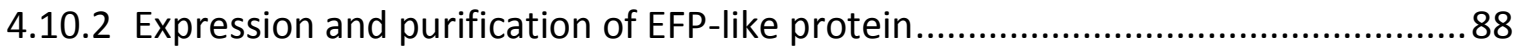

4.10.3 Sodium dodecyl sulfate polyacrylamide gel electrophorese (SDS-PAGE) .............88

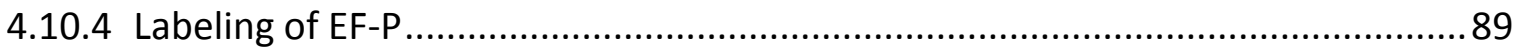

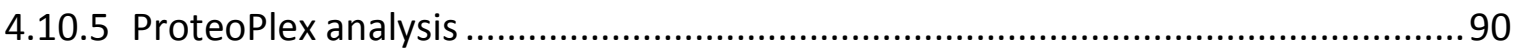

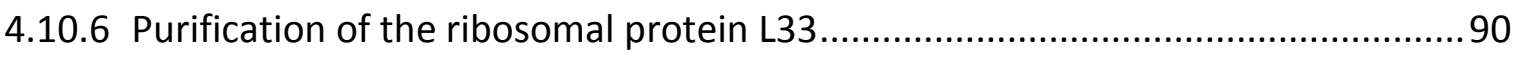

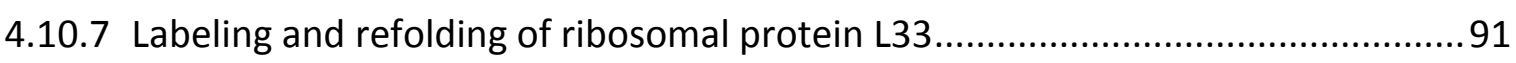

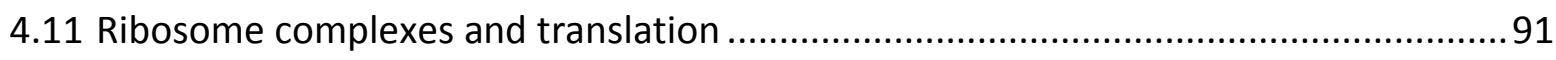

4.11.1 Reconstitution of 50S subunits with the ribosomal protein L33 ......................... 91

4.11.2 Assembly of 70S initiation and post-translocation complexes............................92

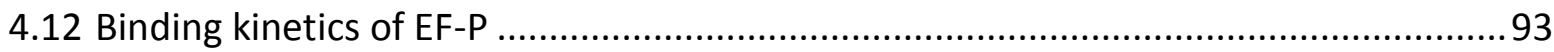

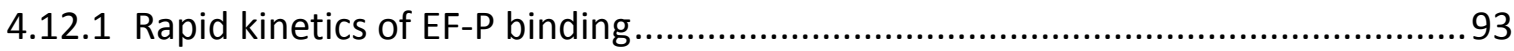

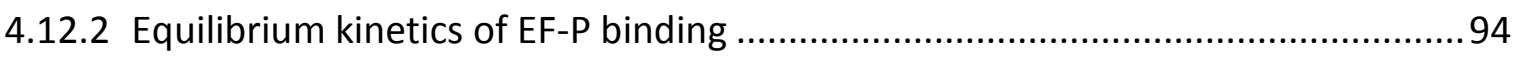

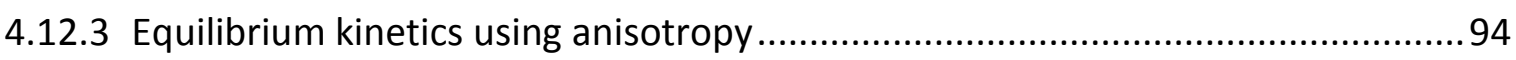

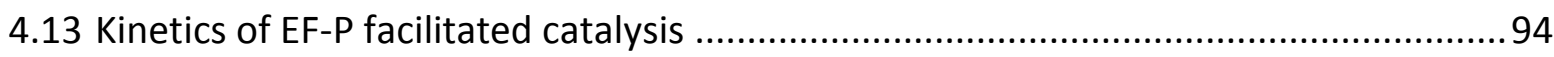




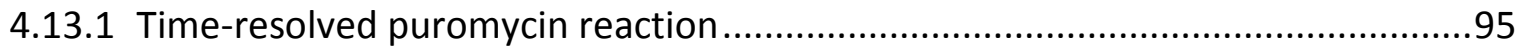

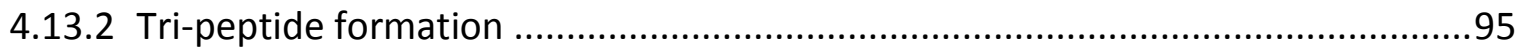

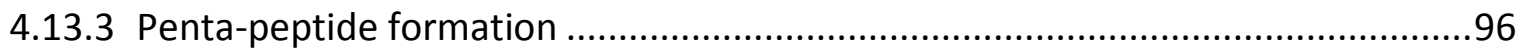

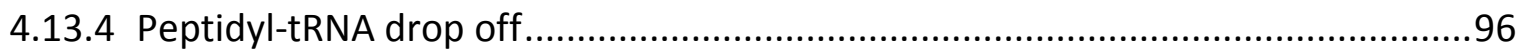

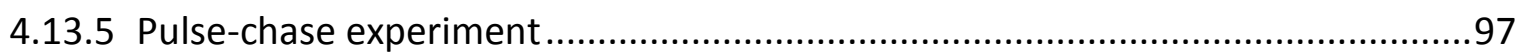

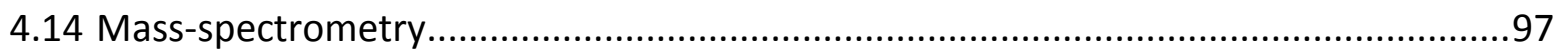

4.14.1 Sample preparation for mass spectrometry …..................................................97

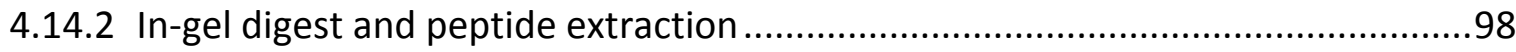

4.14.3 Quantification of intracellular EF-P by mass spectrometry ................................98

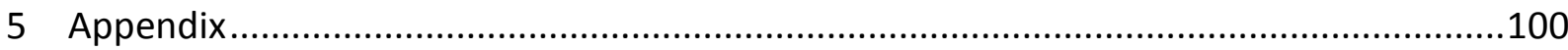

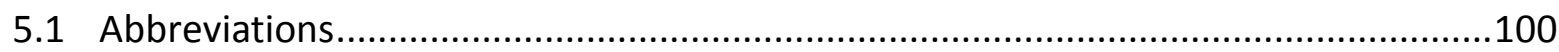

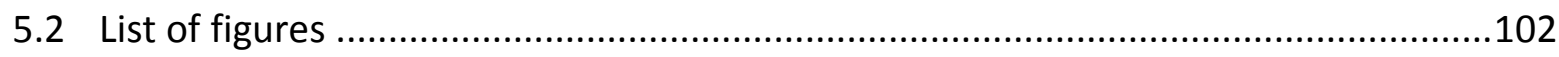

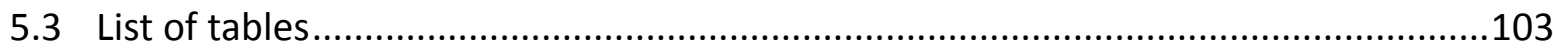

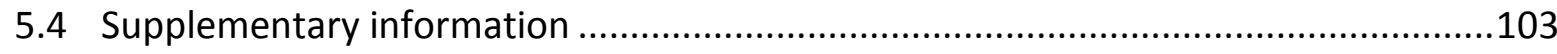

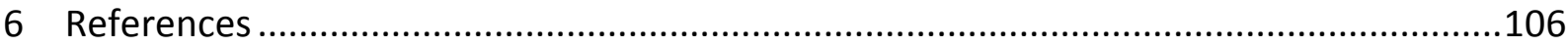

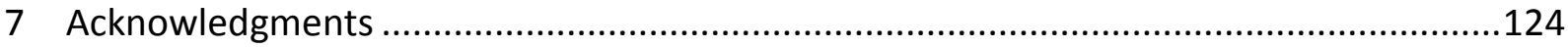

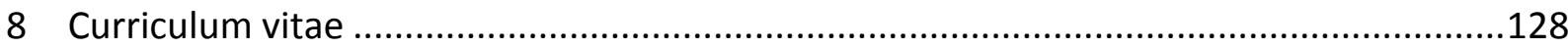





\section{Abstract}

Translation elongation is a multi-step process orchestrated by elongation factors. Elongation factors $\mathrm{G}$ and $\mathrm{Tu}$ are required for each round of translation elongation, whereas elongation factor $P$ is only required to assist the translation of poly(Pro) sequences. Upon incorporation of consecutive proline residues the ribosome is stalled; this stalling is alleviated by EF-P. While the catalytic mechanism of EF-P is well described, the determinants of EF-P binding remain unknown. Structural data and biochemical studies suggest the E-site codon, the peptidyl-tRNA, the ribosomal protein L1 and the post-translational modification of EF-P as key interaction partners during binding and EF-P assisted catalysis.

In this thesis we developed a FRET based EF-P binding assay using fluorescence-labeled ribosome complexes and a quencher-labeled EF-P. We combined the binding assay with different EF-P activity assays to determine the contribution of each of the proposed interactions to the binding and the catalytic activity of EF-P. We found that EF-P binds to different ribosome complexes with similar rates. EF-P has a short residence time on complexes without poly(Pro) stalling sequences, which is significantly increased on poly(Pro)-stalled complexes. This high affinity state depends on the presence of several recognition elements in poly(Pro)-stalled complexes, in particular tRNA ${ }^{\text {Pro }}$ in the $\mathrm{P}$ site and the polypeptide chain containing several sequential proline residues. The contextindependent association rates and the determined cellular concentration of EF-P suggest that the sampling of ribosome complexes by EF-P is kinetically controlled by the availability of a vacant $E$ site. However, only poly(Pro)-stalled ribosome complexes provide the interactions required for the high-affinity binding of EF-P. The dissociation rates from Pro-stalled and non-stalled complexes match the reported rates of EF-P-assisted peptide bond formation. This suggests a mechanism in which the prolonged residence time for stalled complexes allows EF-P to position the peptidyl-tRNA in a catalytically active conformation and thereby to alleviate the stalling. After peptide bond formation the complex returns to the low affinity state, inducing dissociation of EF-P. The proposed kinetic regime allows EF-P to efficiently sample ribosomes with empty E sites, to recognize Pro-stalled complexes with high turnover rates and to alleviate stalling in a single functional cycle. Thus, our work demonstrates that the recruitment of EF-P is kinetically controlled contributing to a harmonized rate of translation. 



\section{Introduction}

A central paradigm of life is to pass the genetic information to one's offspring. This process requires to store genetic information in a conserved way, while still being able to adjust quickly enough to a given stress. The cell achieves this by sustaining information in different forms using either deoxyribonucleic acids (DNA) or ribonucleic acid (RNA). DNA is used for long-term storage of information. DNA is transcribed into RNA. RNAs can be classified in non-coding or coding RNA. Non-coding RNAs either form the functional part of the ribosome (ribosomal RNA, rRNA), adapter molecules (transfer RNA, tRNA) or regulators (small or micro RNA). Coding RNAs, called messenger RNA (mRNA), provide the cell with a quickly accessible and easy to turn-over information source. mRNAs are translated into polypeptides by the ribosome, a macro-molecular machine. Proteins sustain metabolism, replication, motility, the cells infrastructure and architecture of the cell. The nexus of protein biosynthesis lies in the ribosome and its ability to perform translation.

\subsection{Translation - a general overview}

Translation is the process of protein biosynthesis carried out by the ribosome. In prokaryotes the ribosome is assembled by joining of the small with the large subunit, forming an approximately 2.5 MDa ribonucleoprotein complex. The small subunit, which has a sedimentation coefficient of 30 Svedberg units (S) in bacteria consists of 16S rRNA and 21 proteins (S1 to S21). The 30S subunit harbors the decoding center, which allows the decoding of the mRNA codons by tRNAs. The large subunit, with a sedimentation coefficient of $50 \mathrm{~S}$ in bacteria entails $23 \mathrm{~S}$ and $5 \mathrm{~S}$ rRNA, and 31 proteins (L1 to L31). It harbors the peptidyl transferase center (PTC) that facilitates the catalysis of peptide bond formation. Because the catalytic center of the ribosome is formed by the $23 \mathrm{~S}$ rRNA, the ribosome is a ribozyme. The ribosome is constituted of $60 \%$ rRNA and $40 \%$ protein (Ban et al., 2000; Nissen et al., 2000; Noller et al., 1992).

Most tRNAs are 73 to 90 nucleotide long and adopt an L-shape. The tertiary structure allows tRNAs to serve as a connector between the decoding center on the 30S subunit and the PTC on the 50S subunit. Each tRNA features a specific anticodon for the decoding 
of the mRNA codon and a single-stranded CCA-end which carries the corresponding amino acid (aa). The esterification of the aa with the $3^{\prime}$-hydroxyl group of the terminal ribose at the CCA-end of the tRNA is catalyzed by aminoacyl-tRNA synthetases (aa-RS). The high fidelity of this reaction is achieved by aa-RSs due to the recognition of tRNA identity elements in the tRNA sequence (Saks et al., 1994).

In bacteria protein biosynthesis is initiated by the assembly of the $30 \mathrm{~S}$ initiation complex by binding of the initiation factors (IFs) 1,2 \& 3, initiator tRNA (fMet-tRNA ${ }^{\text {fMet }}$ ) and mRNA to the 30 S subunit (Milon and Rodnina, 2012) (Figure 2). The recruitment of the mRNA is independent of the IFs (Milon et al., 2012), however it is modulated by the mRNA secondary structure and the interactions between the Shine-Dalgarno sequence of the mRNA and anti-Shine-Dalgarno sequence in the 16S rRNA (Shine and Dalgarno, 1974; Studer and Joseph, 2006). The formation of the 30 S initiation complex (IC) is completed when fMet-tRNA ${ }^{\text {fMet }}$ recognizes the AUG start codon displayed by the mRNA (Milon et al., 2008). Joining of the $50 S$ subunit triggers the GTPase activity of IF2, causing GTP hydrolysis and resulting in the dissociation of IF1 and IF2. The dissociation of IF3 marks the formation of the translation competent 70S IC (Goyal et al., 2015; Grigoriadou et al., 2007; Tomsic et al., 2000). The translation competent 70S IC provides three binding sites for tRNAs, the acceptor site ( $A$ site), the peptidyl-tRNA binding site ( $P$ site) and the exit site (E site) (Figure 1).

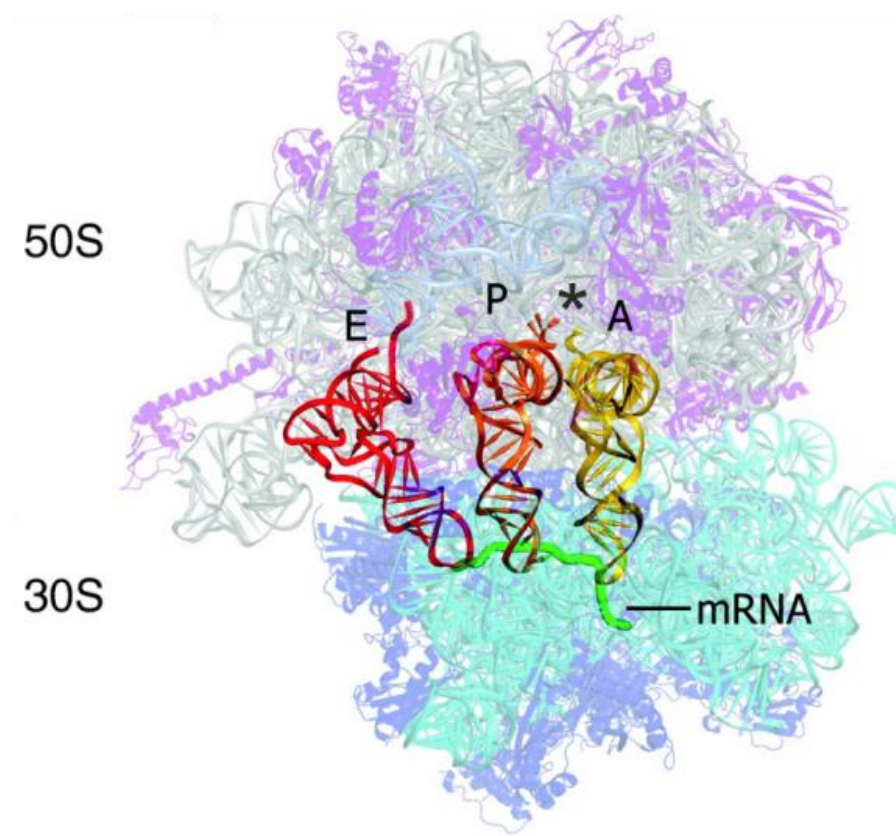


Figure 1: Structure of the 70S ribosome.

The $70 \mathrm{~S}$ ribosome consists of two subunits, the 50S (grey/purple) and $30 \mathrm{~S}$ (blue/turquoise) subunit. The ribosome has three binding sites for tRNAs, the acceptor site (A site, yellow), the peptidyl-tRNA binding site ( $P$ site, orange) and the exit site ( $E$ site, red). The mRNA (green) is bound to the $30 \mathrm{~S}$ subunit. The PTC is marked with a star. Figure adopted from [PDB 4V89] (Zhou et al., 2012).

Translation elongation entails three iterative steps: decoding, peptide bond formation and translocation. The overall rate of protein synthesis is 10 to 25 amino acids per second. This rate is primarily limited by the delivery of the cognate aa-tRNA (Bremer and Dennis, 2008), whereas the rates of peptide bond formation and translocation must be faster. Aminoacyl-tRNAs are delivered to the A site bound to EF-Tu*GTP as ternary complex, which is recruited by the L7/L12 stalk of the ribosome (Diaconu et al., 2005; Kothe et al., 2004). The correct base-pairing between the delivered aa-tRNA and the A site mRNA codon triggers a conformational change of the $30 \mathrm{~S}$ subunit resulting in GTP hydrolysis by EF-Tu and phosphate release (reviewed in: (Rodnina and Wintermeyer, 2016). The EF-Tu*GDP complex has a reduced affinity to aa-tRNA, allowing the accommodation of the aa-tRNA acceptor-end (CCA-end) in the PTC (Kothe and Rodnina, 2006). The accommodated aa-tRNA receives the polypeptide chain (nascent chain) of the P-site peptidyl-tRNA by forming a peptide bond. After peptide bond formation the ribosomebound tRNAs can move from the classic or the hybrid state. In the classic state the peptidyl-tRNA is bound to the $A$ site $(A / A)$ and the deacylated tRNA to the $P$ site $(P / P)$. In the hybrid state the acceptor arms of both tRNAs shift spontaneously to the adjacent tRNA binding sites, occupying the P/A sites and E/P sites (Adio et al., 2015; Fischer et al., 2010; Moazed and Noller, 1989). To continue translation, the ribosome moves along the mRNA by one codon and the tRNAs translocate synchronously to the E site and P site. Translocation is a highly dynamic step and is greatly facilitated by the binding of the elongation factor G (EF-G) and hydrolysis of GTP (Rodnina and Wintermeyer, 2011). EF-G is able to bind to the ribosome independent of the formed tRNA state (Cornish et al., 2008; Holtkamp et al., 2014; Sharma et al., 2016). The EF-G-catalyzed GTP hydrolysis results in a higher flexibility (unlocking) of the 30 subunit by inducing a conformational change. The higher flexibility leads to the relaxation of the ribosome interaction with the codon-anticodon complex allowing the ribosome to move along the mRNA (Belardinelli et al., 2016a; Rodnina et al., 1997; Savelsbergh et al., 2003). The ribosome is now occupied 
by a peptidyl-tRNA in the $P$ site, a deacylated tRNA in the $E$ site from which it spontaneously dissociates, and a vacant A site displaying the next codon.

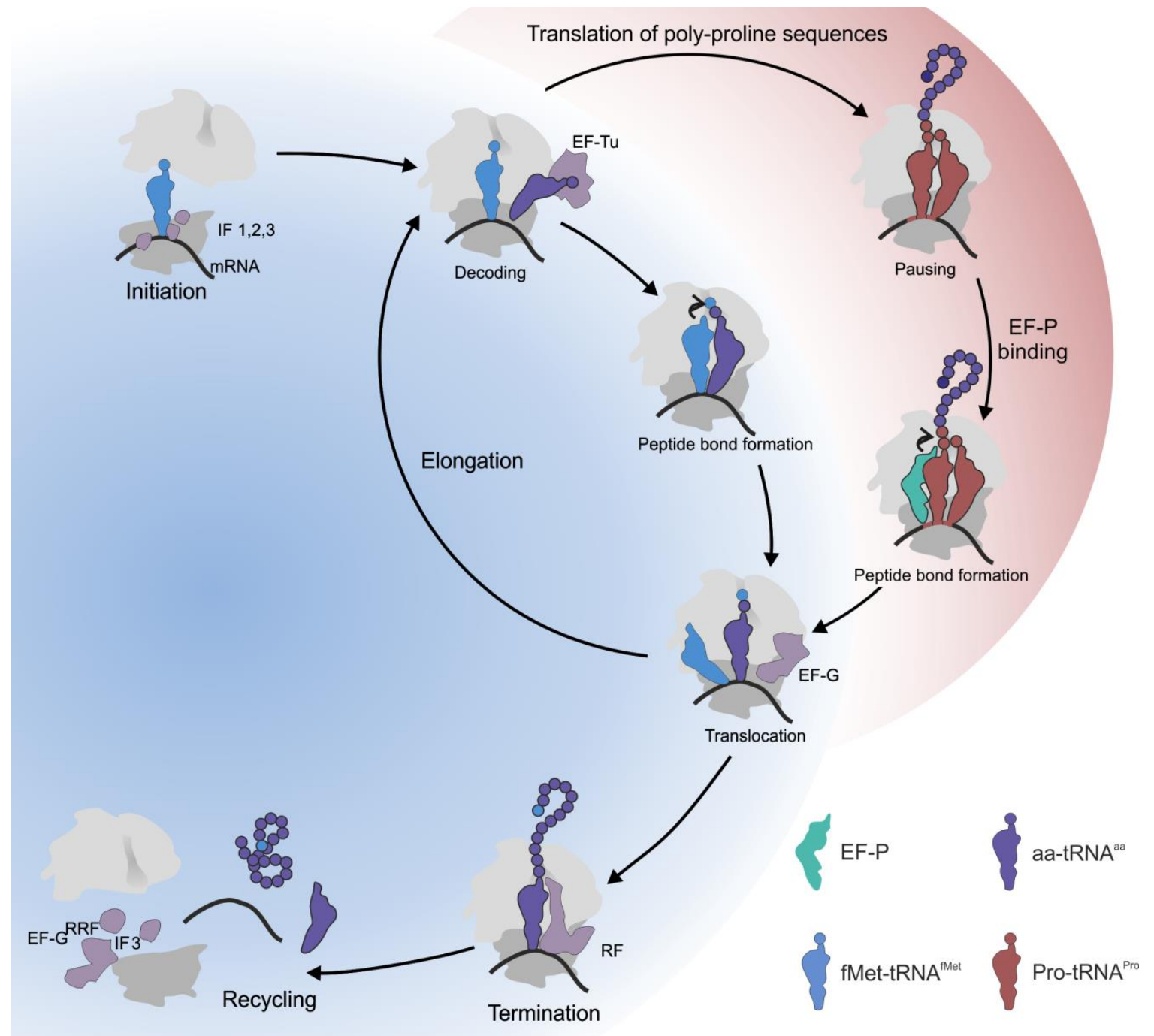

Figure 2: Overview of the translation elongation cycle.

The $30 \mathrm{~S} \mathrm{IC}$ is formed by binding of the initiator tRNA at the mRNA start codon in the P-site of the 305 subunit (dark grey), guided by the initiation factors $1,2 \& 3$. The 70 initiation complex is formed by joining of the 50S (light grey) subunit and the 30S IC (Milon and Rodnina, 2012). Elongation encompasses the repetitive steps of decoding, peptide bond formation and translocation. In the first step EF-Tu*GTP delivers an aa-tRNA to the A site. Upon delivery, a new peptide bond between the polypeptide chain attached to the P-site tRNA and the amino acid of the A-site tRNA is formed. Binding of EF-G promotes translocation causing the tRNA to dissociate from the $E$ site and creating a vacant $A$ site, hence priming the ribosome for the next round of elongation. Upon translation of a sequence encoding multiple consecutive prolines (red background) translation comes to a halt on the second proline codon. The stalling is alleviated by the action of EF-P which facilitates peptide bond formation between the poor substrate Pro-tRNA ${ }^{\text {Pro }}$ (dark red) and other less reactive aminoacyl-tRNAs, allowing translation to continue. Nascent peptide, is released by binding of RF1 or RF2 to the stop codon displayed in the A site inducing the hydrolysis of the ester bond between the nascent chain and the tRNA. After termination, the 70S complex becomes disassembled by an orchestrated action of the ribosome recycling factor IF3 and EF-G. 
Elongation is terminated by release factors that bind to the A site presenting a stop codon. Release factors distinguish their favored stop codons using a tripeptide motif in the respective factor, e.g., RF1 (PXT-tripeptide) reads the UAG and UAA stop codons, whereas RF2 (SPF-tripeptide) reads the UAG and UAA stop codons (Ito et al., 2000). Both RFs share a second functional tripeptide motif (GGQ), in which the Gln is in both cases post-translationally modified (Nakahigashi et al., 2002). The GGQ motif coordinates a water molecule in the PTC, resulting in the hydrolysis of the ester bond between the nascent chain and the tRNA (Dincbas-Renqvist et al., 2000; Shaw and Green, 2007; Weixlbaumer et al., 2008). The dissociation of RF1 and RF2 from the ribosome is facilitated by RF3 hydrolyzing GTP (Adio et al., 2018; Peske et al., 2014). The disassembly of resulting post-termination complex into free tRNA, mRNA and ribosomal subunits is orchestrated by the joined action of EF-G, the ribosome recycling factor (RRF) and IF3. EF-G together with RRF facilitate the dissociation of the ribosomal subunits, whilst IF3 hinders their re-association (Peske et al., 2005) and serves as a bridging factor towards the next round of initiation (Milon and Rodnina, 2012).

\subsection{Peptide bond formation}

Peptide bond formation results from the nucleophilic attack of the $\alpha$-amino moiety of the aa-tRNA on the carbonyl group of the esterified peptidyl-tRNA forming a tetrahedral adduct. The subsequent dissociation of the tetrahedral adduct results in the formation of the peptide bond. The new polypeptide-tRNA becomes longer by one amino acid and the P-site tRNA is deacylated. The ribosome-independent aminolysis reaction in solution is likely to proceed through two tetrahedral intermediates. The nucleophilic attack of the $\alpha$-amino moiety results in the formation of a zwitterionic intermediate $(T \pm)$, consisting of a secondary ammonium cation and an oxyanion. Subsequently, the secondary ammonium ion becomes deprotonated and forms the negatively charged second intermediate (T-) (Figure 3). The decomposition of the second intermediate results in the formation of the reaction products (Satterthwait and Jencks, 1974). Biochemical experiments (Dorner et al., 2003; Kuhlenkoetter et al., 2011; Weinger et al., 2004), structural studies (Schmeing et al., 2005) and molecular dynamic simulations (MDS) (Trobro and Aqvist, 2006; Wallin and Aqvist, 2010) suggested a proton-shuttle mechanism for the ribosome catalyzed reaction. 
The proton of the attacking nucleophile is abstracted by the 2'-hydroxyl group and is shuttled to the 3'-hydroxyl group of the corresponding P-site tRNA. The proton-shuttle relies either on one or two additional water molecules, resulting in an 8-membered or 10-member proton-shuttle, respectively (Kazemi et al., 2018; Rodnina, 2013). A structural study, suggested a second water molecule in close proximity to the ribosomal protein L27 (Polikanov et al., 2014). Interestingly computational simulations suggested that both shuttle models are in agreement with previous kinetic solvent isotope effect studies (KSIE) for peptide bond formation (Kazemi et al., 2018; Kuhlenkoetter et al., 2011). A heavy-atom kinetic isotope effects (KIE) analysis revealed that the breakdown of the tetrahedral intermediate is not part of the rate-limiting step, unlike its formation and the proton transfer from the attacking nitrogen (Hiller et al., 2011). The function of the ribosome is to lower the activation entropy and change the rate limiting transition state (reviewed in: (Rodnina, 2013).
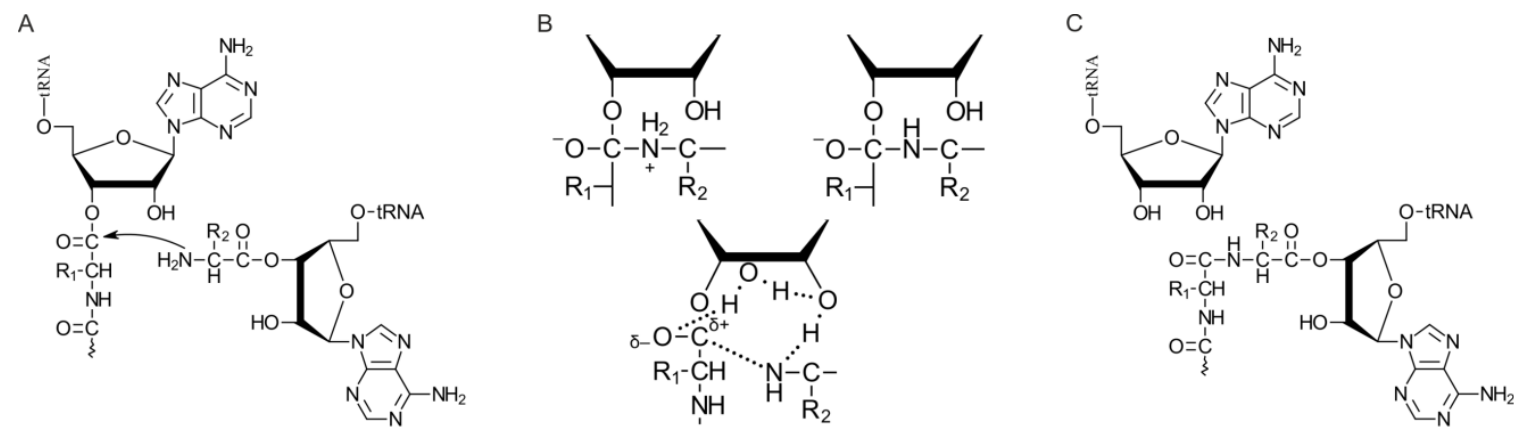

Figure 3: Comparison of peptide bond formation in solution and catalyzed by the ribosome.

A) P-site peptidyl-tRNA and A-site aa-tRNA depicted prior to peptidyl transfer in solution, with the imminent nucleophilic attack indicated by an arrow.

B) The top-raw depicts the proposed transition states ( $T \pm$ and $\mathrm{T}-$ ) of the in-solution reaction, whereas the bottom raw shows the ribosome catalyzed-reaction with a concerted 8-member proton shuttle.

C) P-site deacylated tRNA and the newly formed A-site peptidyl-tRNA after peptide bond formation.

The catalysis of peptide bond formation by the ribosome is not restricted to L-amino acids, but also allows some D-amino acids and a broad spectrum of unnatural amino acids as substrates (Fujino et al., 2013; Katoh and Suga, 2018; Kawakami and Murakami, 2012; Liu and Schultz, 2010). Considering the limited variety of potential functional moieties available in the peptidyl-transferase center, which is formed by the $23 \mathrm{~S}$ rRNA only, the spectrum of substrates is broad and versatile. The rate of peptide bond formation across the 20 canonical amino acids is rather uniform, with few exceptions such as proline. The 
substrates do show different reaction rates with puromycin, an aminonucleoside antibiotic that resembles the CCA-end of a tRNA and therefore serves as acceptor of the nascent chain. The uniformity of peptide bond formation is achieved by having a preceding rate limiting step, the accommodation of the A-site tRNA (Ledoux and Uhlenbeck, 2008; Wohlgemuth et al., 2008), with very few exceptions and proline being one of them (Pavlov et al., 2009).

In addition to catalysis of peptide bond formation, the ribosome can facilitate the formation of esters, thioesters, thioamides or phosphinoamides (Bieling et al., 2006; Fahnestock et al., 1970; Lieberman and Dahlberg, 1995; Victorova et al., 1976). Hence, the ribosome can be considered as a versatile catalyst.

\subsection{Elongation factor $P$}

\subsubsection{Amino acid proline}

Although the rates for peptide bond formation during translation are in most cases uniform, the rate of translation in the cell can vary substantially. The translation rate is influenced by the abundance of tRNAs, secondary structures of the mRNA or the nature of the amino acids to be incorporated into the growing nascent chain (Bullwinkle and Ibba, 2016; Moine et al., 1988; Pavlov et al., 2009; Yanofsky and Horn, 1994). The nature of the amino acids that react in the PTC to form a peptide bond can slow down and in special cases cause stalling of translation (Ito et al., 2011). Upon translation of consecutively encoded prolines, the ribosome is stalled, as proline is a poor donor and a poor acceptor in peptidyl transfer (Doerfel et al., 2013; Muto and Ito, 2008; Wohlgemuth et al., 2008). The pyrrolidine ring of proline that spans the $\alpha$-carbon and amino group restricts the $\mathrm{N}-\mathrm{C}_{\alpha}$ bond torsion angles to $\phi=-63$ and $\psi=-75$, thus limiting the number of accessible conformations (Hovmoller et al., 2002). Proline also restricts the backbone conformation of the neighboring residues (MacArthur and Thornton, 1991). The pyrrolidine ring can adopt two distinct conformations with $C_{\gamma}$ in an endo- (pointing towards $C_{\alpha}$ ) or exo-conformation (pointing away from $C_{\alpha}$ )(Ramachandran et al., 1970). As other proteinogenic amino acids, proline can adopt two distinct isoforms, cis or trans. While other amino acid sterically favor trans-conformations, proline has nearly no thermodynamic hindrance for a cis-trans-isomerization $(\Delta \mathrm{G} \sim 0.7 \mathrm{kcal} / \mathrm{mol}$ of difference) 
(Owens et al., 2007). The isomerization is, however, so slow and kinetically unfavorable (Fischer et al., 1994), that less than 10\% amino acid-proline bonds in proteins adopt a cis-conformation (reviewed in: (Yaron and Naider, 1993)).

A<smiles>[X]C(=O)[C@H]1[B][13CH][13CH]N1C([R])=O</smiles><smiles>[X]C(=O)C1CCCN1C([R])=O</smiles>

B

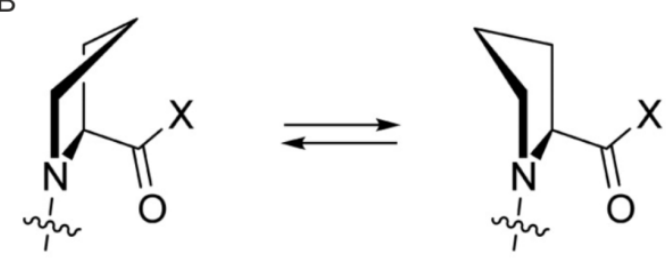

Figure 4: Steric properties of proline.

A) Trans-cis isomerization of proline, with $\mathrm{R}$ and $\mathrm{X}$ representing the preceding and the following amino acid residues.

B) Stereoisomers of proline in the endo- and exo-conformation.

Figure adopted from: (Doerfel et al., 2015).

These characteristics make proline a special amino acid that plays specific roles in proteins. Because proline is sterically restricted and has a limited ability to form hydrogen bonds, it is usually only found in the first turns of $\alpha$-helices (MacArthur and Thornton, 1991; Woolfson and Williams, 1990) and bulges or sheet edges of ß-sheets, where no hydrogen bond donor is required (Li et al., 1996). When located in an $\alpha$-helix, proline can affect the overall structure of the protein. Proline introduces a kink into the secondary structure, which, because of prolines rigidity can translate into the tertiary structure (Barlow and Thornton, 1988). Hence, prolines are frequently found in irregular structures such as $\alpha$-helical capping motifs, $\beta$-turns, poly(Pro)- and collagen-helices (Bhattacharyya and Chakrabarti, 2003; Chakrabarti and Pal, 2001; MacArthur and Thornton, 1991). The puckering of proline's pyrrolidine ring allows it to tune the stability of the secondary structure, as demonstrated e.g. for collagen-helices (Vitagliano et al., 2001). The cis-trans-isomerization of proline in proteins can confer to a hinge like function, allowing major conformational changes of folded proteins. This mechanism was reported for the opening of channels (Lummis et al., 2005), isomer-dependent dimerization (Jenko Kokalj et al., 2007; Solbak et al., 2010), membrane binding (Evans and Nelsestuen, 1996) and auto-inhibition control (Sarkar et al., 2007). Cis-trans-isomerization occurs slowly and was reported to be modulated by intra- and intermolecular interactions (reviewed in: (Andreotti, 2006)). 
During peptide bond formation proline is a particular poor substrate, as donor as well as acceptor. The resulting slow peptide bond formation becomes an obstacle for the translation of poly(Pro) sequences. The reaction between the peptidyl-Pro-Pro-tRNA in the $\mathrm{P}$ site and the Pro-tRNA in the A site is slowed down to an extend that translational stalling occurs. In bacteria the translational stalling caused by a strung of prolines is alleviated by elongation factor P (EF-P) (Doerfel et al., 2013; Ude et al., 2013). Because poly(Pro) sequences are ubiquitous, homologs of EF-P are found in eukaryotes (elF5a) and archaea (alF5a). One of these poly(Pro)-containing proteins is the valine tRNA synthetase (ValS), which was found in genomes of over 1200 bacterial, 205 archaeal and nearly 100 eukaryotes (Starosta et al., 2014b). The proline-triplet of ValS is located in the active center. It is essential for efficient Val-tRNA ${ }^{\mathrm{Val}}$ charging and prevents mischarging of tRNA $^{\text {Val }}$ with threonine (Fukai et al., 2000; Fukai et al., 2003). As the valS gene is essential for cell viability and the presence of EF-P is a prerequisite for efficient valS translation, it has been suggested that both proteins co-evolved (Baba et al., 2006; Starosta et al., 2014b).

\subsubsection{Function and biological relevance of EF-P}

EF-P was initially identified by its ability to increase the product yield of an $\mathrm{fM}$-puromycin reaction (Glick and Ganoza, 1975). Shortly after, EF-P was reported to enhance the peptidyl-transferase activity for glycine, stimulate poly(Phe)/(Lys) product formation and the translation of a naturally occurring mRNA (Aoki et al., 1997; Aoki et al., 2008; Ganoza et al., 1985; Glick et al., 1979; Glick and Ganoza, 1975; Green et al., 1985). However, these reported effects were small and did not exceed a factor of 2-fold. Based on a structural study and the described biochemical insights, it was proposed that EF-P either positions the tRNA ${ }^{\text {fMet }}$ in a productive conformation in the P-site (Aoki et al., 2008; Blaha et al., 2009) or facilitates the initial peptide bond formation (Aoki et al., 2008; Blaha et al., 2009). Two research groups simultaneously elucidated the functional context of EF-P (Doerfel et al., 2013; Ude et al., 2013). Doerfel, Wohlgemuth and colleagues used a fully reconstituted in vitro translation system to calculate the reaction rates between different amino acids in the P site of the PTC and puromycin as a mimic of aa-tRNA in the A site. 
A $\quad$ M1 M2 M7 $0 \quad 10 \quad 30 \quad 50100150200250$ - M1 M2 M7 $0 \quad 10 \quad 3050100150200250$

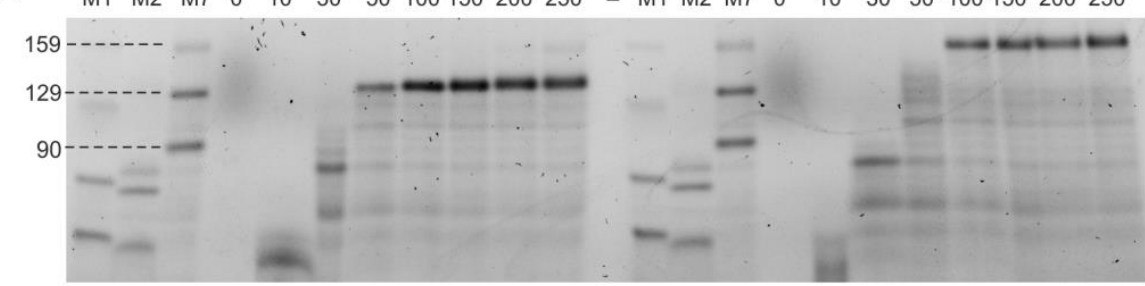

B

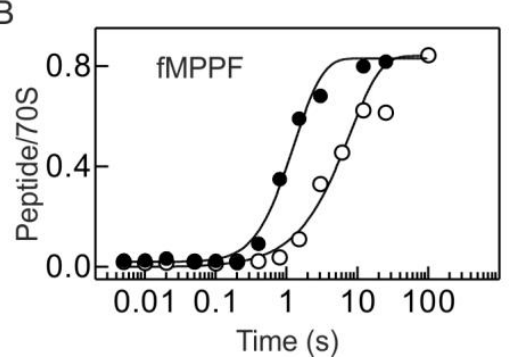

C

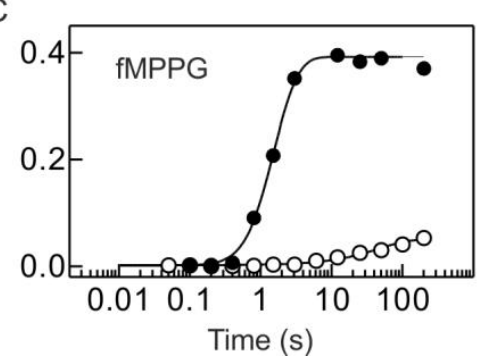

D

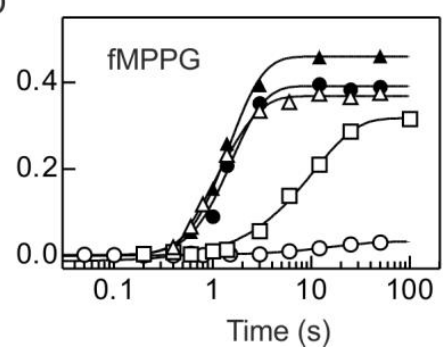

Figure 5: EF-P facilitates synthesis of poly-proline and PPG-containing peptides.

A) Translation of AmiB containing a poly(Pro) sequence in the absence (left side) or presence of EF-P (right side).

B \& C) Formation of a model peptide in a fully reconstituted in vitro translation system in the absence (white circles) or presence of EF-P (black circles).

D) Contribution of EF-P K34 modification to the formation of FMPPG peptide in a fully reconstituted in vitro translation system. Reaction facilitated by: unmodified EF-P (open squares), overexpressed lysinylated but unhydroxylated EF-P (solid triangles), and lysinylated/hydroxylated overexpressed EF-P (solid circles), native EF-P (open triangles) or no EF-P (open circles).

(Figures adopted from: (Doerfel et al., 2013))

They found that for most P-site amino acids EF-P stimulated the puromycin reactions by only about 5-fold, except with fMP with which the reaction was accelerate 90-fold. Similar effects were observed for the incorporation of different aa-tRNA A site substrates. The formation of di-peptides was, however, accelerated to a smaller extend. For most combinations, EF-P facilitates peptide bond formation 2-fold. However, for poorly reactive substrates like glycine or proline larger effects were observed, 8-fold and 16-fold, respectively. Longer poly(Pro) model peptides (e.g. fMPPG or fMPPPF) were not synthesized in the absence of EF-P. Interestingly, the amino acid after the PP sequence also has an impact on the translation efficiency (Figure 5: B vs C). Later experiments have confirmed this notion and have shown that also the downstream amino acid can affect translation efficiency. This allows to group amino acid sequences into strong stallers (PPP, DPP or PPN) or weak stallers (CPP, PPR or PPH) (Doerfel et al., 2013; Peil et al., 2013; Starosta et al., 2014a; Ude et al., 2013). Using natural, substantially longer mRNA sequences in the in vitro translation system led to similar results: translation of the AmiB 
fragment (159 aa), which contains eight consecutive prolines, was negligible in the absence of EF-P, but very efficient when EF-P was added (Figure 5A). Residue lysine 34 of EF-P is post-translational modified to (R)- $\beta$-lysylhydroxylysine (Navarre et al., 2010; Peil et al., 2012; Roy et al., 2011; Yanagisawa et al., 2010) and the modification is important for EF-P function (Figure 5D). Overexpressed lysinylated/hydroxylated, lysinylated, but not hydroxylated, and native EF-P have similar activity. Unmodified EF-P showed a significantly reduced activity, whereas without EF-P nearly no product formation was observed (Doerfel et al., 2013). In addition to unmodified EF-P, Ude and co-workers created the EF-P variant $\mathrm{K} 34 \mathrm{~A}$, which cannot be modified. In their in vitro translation system unmodified EF-P and EF-P K34A showed no activity, and yielded the same readout as a reaction without EF-P (Ude et al., 2013). Thus, the presence of EF-P and its post-translational modification is indispensable for synthesis of poly(Pro) containing proteins (Doerfel et al., 2013; Peil et al., 2013; Ude et al., 2013).

Apart from facilitating peptide bond formation, EF-P was also reported to stabilize the P-site peptidyl-tRNA (Doerfel et al., 2013). This was reflected in higher product yields resulting from a reduced drop-off of peptidyl-tRNA from the ribosome. The stabilizing effect of EF-P on the peptidyl-tRNA was further supported by a cryo-electron microscopic study, which reported a high abundance of vacant ribosomes in the absence of EF-P (Huter et al., 2017).

As described in chapter 1.2 the ribosome can incorporate some D-amino acid into a growing nascent chain, but incorporation of several consecutive D-amino acids was described to be almost impossible. Because EF-P can facilitated peptide bond formation and resolve stalling resulting from the consecutive incorporation of L-Pro, it was suggested that EF-P could facilitated peptide bond formation for D-amino acids. Indeed, EF-P was reported to increase the relative poly-D-Phe yield from 1 to $10 \%$ (Huang, 2017). However, the proposed mechanism of EF-P binding to the D-amino acid containing P-site peptidyl-tRNA and subsequent facilitation of peptide bond formation, is in disagreement with (Katoh et al., 2016). Katoh and co-workers could show that the presence of the tRNA ${ }^{\text {Pro }} \mathrm{D}$-arm architecture is a requisite for EF-Ps activity, whereas Huang and co-workers used a tRNA ${ }^{\text {Glu2 }}$. Furthermore, EF-P-facilitated poly-D-Phe synthesis failed to be reproduced in a later study (Katoh et al., 2017a), supporting the relevance of the tRNA D-arm. Optimization of the reaction conditions resulted in an increased incorporation of 
consecutive D-amino acids by as much as 5-fold in the absence of EF-P (Katoh et al., 2017b). To further improve the efficiency of D-amino acids incorporation, the D-arms of their respective tRNAs were engineered to be recognized by EF-P, which improved the yield by as much as 10-fold (Katoh et al., 2017a). The same approach was recently used to achieve the consecutive incorporation of $\beta$-amino acids for the first time (Katoh and Suga, 2018). These finding indicate a potential of EF-P for biotechnological and pharmaceutical applications, which would allow the more efficient synthesis of peptides containing non-proteinogenic amino acids.

The relevance of EF-P was also shown in in vivo studies. Despite EF-Ps crucial function in translation, addition functions have been reported. EF-P was proposed to ensure the coupling of the translating ribosome to the transcribing RNA polymerases by preventing ribosome stalling induced by poly(Pro) sequences (Elgamal et al., 2016). Hence, EF-P can not only regulate protein biosynthesis on the translational level, but also indirectly on a transcriptional level. However, the number of genes that require EF-P for maintaining the coupling of transcription and translation appears to be rather low (reviewed in: (Rajkovic and Ibba, 2017)). Furthermore, EF-P was reported to prevent frameshifting. In the absence of EF-P, spontaneous frameshifting was increased 3-fold in vivo (Gamper et al., 2015). However, it was neglected that $\Delta e f p$ variants in general showed a 2- to 3-fold reduced translation capacity (Hersch et al., 2013). In vitro experiments in the absence of EF-P suggested a 30-fold increase in frameshifting efficiency (Gamper et al., 2015). Such acceleration of a proline independent reaction by EF-P, is however, in conflict with previously reported rates (Doerfel et al., 2013).

Interestingly, EF-P does not seem to be essential for many bacteria. In E. coli it appears to be conditionally essential, since both cases have been reported (Aoki et al., 1997; Baba et al., 2006; Gerdes et al., 2003; Joyce et al., 2006; Peil et al., 2013; Peil et al., 2012; Yamamoto et al., 2009). For many other bacteria, e.g. Bacillus, Salmonella, Shigella, Pseudomas, Agrobacterium or Acinetobacter, EF-P is not essential for cell viability (Balibar et al., 2013; de Crecy et al., 2007; Langridge et al., 2009; Peng et al., 2001). However, upon deletion of the efp gene or genes encoding the post-translational modifying enzymes, severe phenotypes can be observed (Abratt et al., 1998; Van Dyk et al., 1987). In addition, many pathogenic bacteria become significantly less or avirulent. Variants (efp, epmA and epmB) of different Salmonella species showed a hyper-susceptibility towards 
low osmolality, various antibiotics, an attenuated virulence and reduced migration abilities, resulting from perturbations in the cell envelop (Bearson et al., 2011; Block et al., 2010; Choi et al., 2018; Hersch et al., 2013; Kaniga et al., 1998; Navarre et al., 2010; Zou et al., 2012). Shigella flexneri poxA and efp variants showed an impaired ability to form plagues or invade the host epithelial cells. Interestingly, not only virulence proteins were found to be reduced, but also the mRNA level of a master regulator of virulence were lower (Marman et al., 2014). Brucella aboruts variants lacking EF-P failed to invade their host cells (Iannino et al., 2012). Neisseria meningitidis appears to require rhamonslyated EF-P for its cell viability (Yanagisawa et al., 2016). Aside from human pathogens, also plant pathogens show reduced virulence and higher susceptibility upon deletion of EF-P and its modifying enzymes. Deleting the EF-P homolog encoded by $c h v H$ in the plant pathogen A. thumefaciens produced variants defective in plant virulence (Charles and Nester, 1993). In Erwinia amylovora the deletion of epmB is sufficient to cause a reduced virulence and an increased chemical sensibility (Klee et al., 2018). The reduction of pathogenicity and increased stress susceptibility, in bacteria with an impaired EF-P activity, makes EF-P and its modifying enzymes to a putative drug target. Because the analogous system in higher eukaryotes is highly specific, it is unlikely to be targeted by an inhibitor of the bacterial system. Taken together, these findings make EF-P and its modifying enzymes a promising target to develop novel antimicrobials.

\subsubsection{Structure of elongation factor $P$}

EF-P consists of three domains resembling the L-shape of tRNAs (Figure 6A \& B). It has an overall negative net charge, except for the tip of domain I (Hanawa-Suetsugu et al., 2004). The eukaryotic and archaeal initiation factor 5A (elF5A and alF5A) are homologs of EF-P. elF5A and alF5A adopted a two domain structure and lack the bacterial specific domain III. Overall, the eukaryotic and archaeal EF-P homologs show a significant structural similarity (Figure 6C and D). 
A

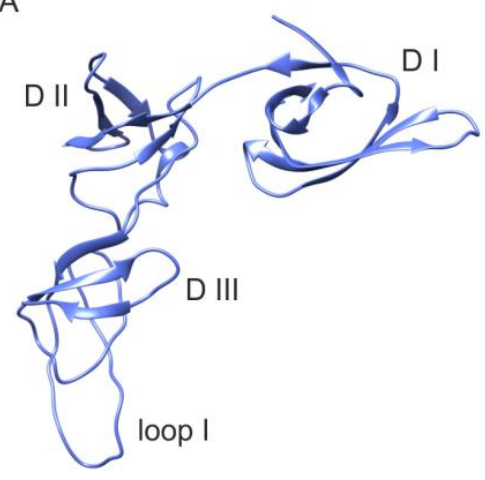

B

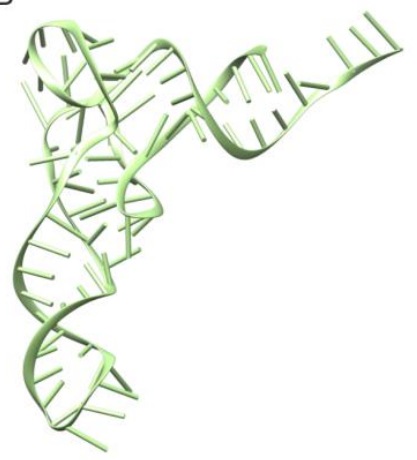

C

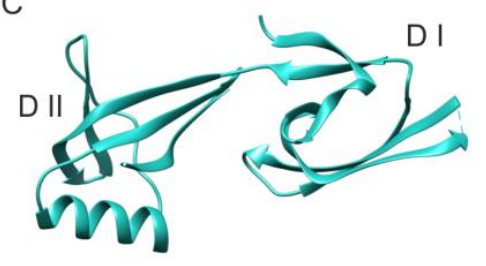

$\mathrm{D}$

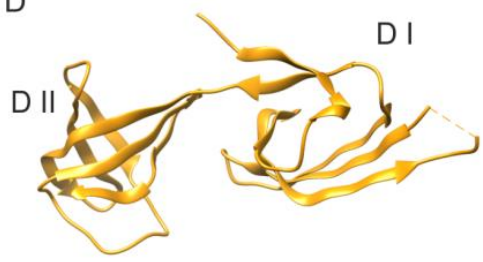

$\mathrm{E}$

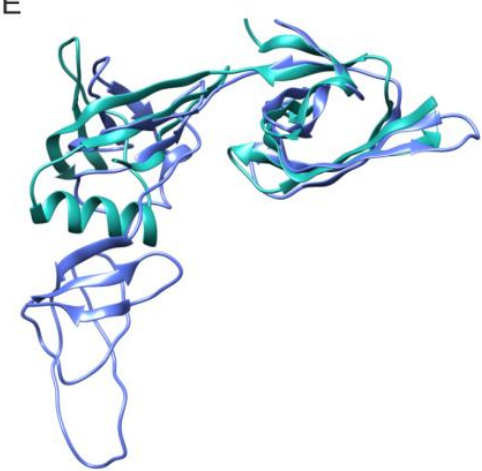

Figure 6: Structural comparison of EF-P, tRNA, elF5A and alF5A.

A) E. coli EF-P obtained by cryo-electron microscopy [6ENU] (Huter et al., 2017) with domains indicated as domain I (DI), domain II (DII) and domain III (DIII).

B) E. coli tRNA ${ }^{\text {Pro1 }}$ obtained by cryo-electron microscopy [6ENU] (Huter et al., 2017).

C) Human elF5A obtained by X-ray crystallographic [3CPF] (Tong et al., 2009b). Labeling as A.

D) Methanocaldococcus jannaschii alF5A obtained by X-ray crystallographic [1EIF] (Kim et al., 1998). Labeling as for A.

E) Superposition of domains I of E. coli EF-P and human elF5A.

The N-terminal domain I of EF-P comprises a ß-barrel fold comprising 6 ß-strands and a flexible loop (loop I) at the tip. In E. coli loop I of domain I is conserved and contains lysine residue 34, which is post-translationally modified. The modification of K34 by $R$-( $\beta)$-Iysinylation, which is crucial for the function of EF-P, is located at the tip of the loop I extending away from the domain body. Crystallographic studies indicate a great flexibility of the linkage between domain I and domain II. Domain II is formed by a ß-barrel with $7 \beta$-strands. It has an oligonucleotide-binding fold, as found in RNA-binding proteins (Blaha et al., 2009; Hanawa-Suetsugu et al., 2004). The link between domain II and domain III appears, compared to domain I - domain II linkage, relative rigid (Choi and Choe, 2011). In EF-P domain III is structurally similar to domain II. The overall domain arrangement mimics the L-shape of a tRNA. A potential key feature of domain III is the flexible loop I containing a conserved GDT motif (Huter et al., 2017). Domain III is specific 
for bacteria and hence lacking in elF5A and alF5A. The N-terminal domain of alF5A and elF5A topologically coincides with EF-P domain I (Figure 6E). Interestingly, the C-terminal domain of a/elF5A is similar to the N-terminal half of EF-P domain II and the C-terminal half of EF-P domain III (Blaha et al., 2009). EF-P domain III appears to originate from a duplication of EF-P domain II. However, the evolutionary origin of EF-P domain III and the C-terminal domain of alF5A/elF5A is yet not fully understood (Hanawa-Suetsugu et al., 2004).

\subsubsection{Post-translational modification of EF-P}

The post-translational modification of EF-P K34 was initially recognized by a mass shift of +144 Da (Aoki et al., 2008). Genetic studies suggested a post-translation modification of K34 by $(R)$ - $\beta$-lysine $(+128 \mathrm{Da})$ required two enzymes, EpmA and EpmB (Figure 7A) (Bailly and de Crecy-Lagard, 2010). First, the lysine 2,3-aminomutase (EpmB, also referred to as YjeK) transforms (S)- $\alpha$-lysine into ( $R$ )- $\beta$-lysine (Behshad et al., 2006; Roy et al., 2011). Second, the Elongation factor P-(R)- $\beta$-lysine ligase (EpmA, also referred to as GenX, PoxA and YjeA) ligates (R)- $\beta$-lysine to EF-P K34 in an ATP-dependent manner. This mechanism was confirmed by genetic (Ambrogelly et al., 2010; Navarre et al., 2010) and structural work (Sumida et al., 2010), and by biochemical in vitro assays (Yanagisawa et al., 2010). EpmA evolved from a class II amino acyl tRNA synthetase (aaRS) which lost its anticodon binding domain, gained an EF-P specificity and has a broad substrate spectrum (Bailly and de Crecy-Lagard, 2010; Katz et al., 2014; Navarre et al., 2010; Yanagisawa et al., 2010). In addition to the target substrate, $(R)$ - $\beta$-lysine, in vivo studies showed that EpmA can equally well utilize the enantiomer (S)- $\beta$-lysine, which in fact leads to an activated EF-P (Gilreath et al., 2011; Roy et al., 2011). In addition, in an in-vitro assay also the constitutional isomer ( $L$ )- $\alpha$-lysine was accepted and ligated to K34 of EF-P. However, the a-lysinylated EF-P is inactive (Gilreath et al., 2011; Roy et al., 2011). Notably, in a substrate competition assay $(R)-\beta$-lysine $\left(\mathrm{K}_{\mathrm{M}}=213 \mu \mathrm{M}\right)$ is highly favored over its $\alpha$-lysine constitutional isomer $\left(\mathrm{K}_{\mathrm{M}}=8600 \mu \mathrm{M}\right)$ and its enantiomer $\left(\mathrm{K}_{\mathrm{M}}=6950 \mu \mathrm{M}\right)$ (Roy et al., 2011). The discrepancy of $+16 \mathrm{Da}$ between the mass shift $+144 \mathrm{Da}$ and $+128 \mathrm{Da}$ by $(R)-\beta$-lysine corresponds to the addition of a single oxygen atom, likely, in the form of a hydroxyl moiety. Elogation factor $\mathrm{P}$ hydroxylase (EpmC, also referred to as $\mathrm{YfcM}$ ) was 
reported to hydroxylate K34 of EF-P. YfcM binds only lysinylated EF-P and modifies it at the $\delta$-C5 of K34 (Kobayashi et al., 2014a; Kobayashi et al., 2014b; Peil et al., 2012).

Because EF-P is universally conserved and its modification is of key functional importance (Doerfel et al., 2013; Navarre et al., 2010; Ude et al., 2013; Yanagisawa et al., 2010), it is surprising that only $22 \%$ of all sequenced bacterial genomes encode orthologs of EpmA or EpmB (Bailly and de Crecy-Lagard, 2010; Lassak et al., 2015). A phylogentic analysis revealed that in $9 \%$ of all sequenced bacterial genomes, a conserved arginine residue (R32) is found in a position equivalent to K34 (Choi and Choe, 2011; Lassak et al., 2015). In Shewanella oneidenis, an EF-P R32 modifying enzyme was identified. The EF-P R32 rhamnosyl-transferase (EarP) uses dTDP- $\beta$-(L)-rhamnose as a substrate; the modification of EF-P R32 with rhamnosylation was confirmed by mass-spectrometry (Figure 7B) (Lassak et al., 2015; Wang et al., 2017). Anti-R32-rhamanosyl antibodies and crystallographic studies confirmed the EF-P-EarP interactions in multiple bacterial strains (Krafczyk et al., 2017; Li et al., 2016; Sengoku et al., 2018). In contrast to the EF-P lysinylation mechanism, the usage of dTDP-(L)-rhamnose competes with many other intracellular biosynthetic pathways (Babaoglu et al., 2003; Lam et al., 2011; Lindhout et al., 2009; Rahim et al., 2001; Schirm et al., 2004). Therefore, the flow and the availability of substrate has to be under tight regulation to ensure the efficient modification of EF-P R32. 
A

(S)- $\alpha$-lysine<smiles>NCCCC[C@H](N)C(=O)O</smiles><smiles>[Y][13CH][10BH2]</smiles>

(R)- $\beta$-lysine<smiles>NCCC[C@H](N)CC(=O)O</smiles><smiles>CC1CCCCCC1[Mg]</smiles>

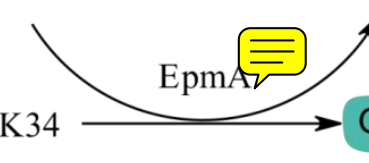<smiles>CC(C)(C)C(=O)C(CC=C[C@H](O)CNC(=O)C[C@@H](N)CCCN)NS(C)(=O)=O</smiles><smiles>CCC(C)C</smiles>

B

dTDP-(L)-rhamonosyl

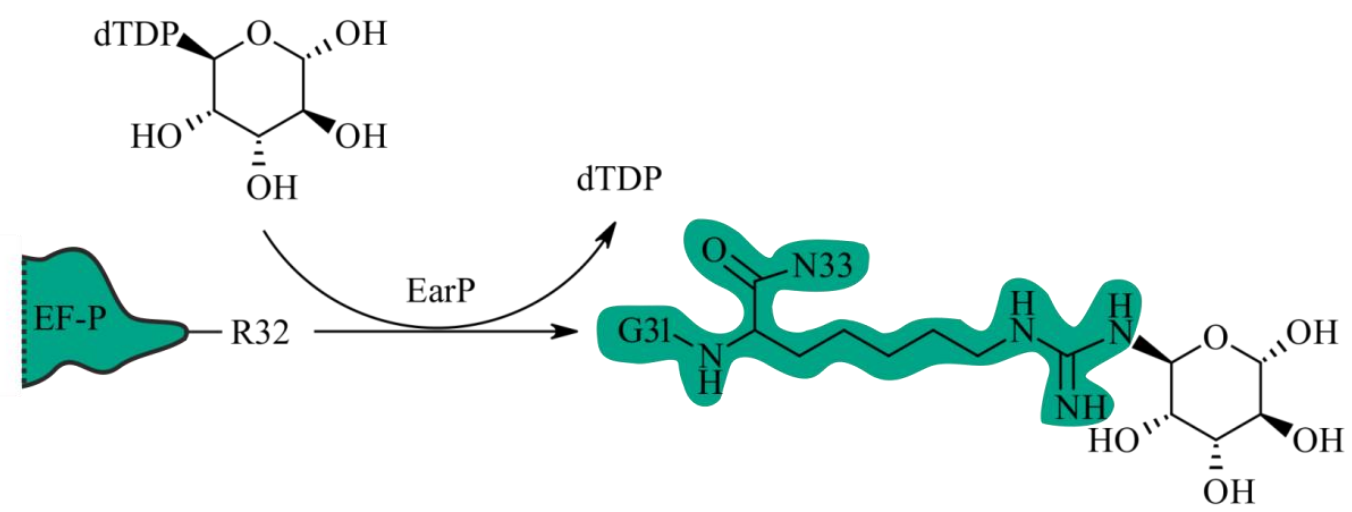

Figure 7: Post-translational modification of EF-P.

A) E. coli EF-P residue $\mathrm{K} 34$ modified to $\varepsilon(R)$ - $\beta$-lysylhydroxylysine by action of EpmA and C.

B) $S$. oneidenis EF-P rhamnosylated at residue R32 by EarP.

The described bacterial EF-P post-translational modification machineries can only be found in $31 \%$ of all sequenced bacterial genomes and mainly in genomes of Gram-negative bacteria. Recently the post-translational modification of EF-P in the Gram-positive model organism Bacillus subtilis was identified. The modification of K32 with 5-aminopentanol showed a positive impact on the translation of poly(Pro) containing proteins. In B. subtilis EF-P-dependent peptide sequences were mainly found in flagellar genes; deletion of EF-P caused a reduced swarming motility. Initially reported sporulation failures (Meeske et al., 2016; Ohashi et al., 2003), could not be restored by complementation studies. In contrast to the previously described modification, the genes 
encoding the modification enzymes were not found in the direct neighborhood to the EF-P gene (Rajkovic et al., 2016), which makes it much harder to identify putative modifying enzyme from a genetic screening. The $B$. subtilis EF-P modifying enzymes Ymfl was shown to catalyze the reduction of 5-aminopentanone to 5-aminopentanol. Interestingly, a 5-aminopenatnone modified EF-P appear to be biochemically inactive, whereas the unmodified EF-P remained active (Hummels et al., 2017). A genetic screen revealed two other genes ( $y n b B$ and $g s a B)$ involved in the modification, however, their direct contribution to a distinct modification step could not be assigned yet. In the corresponding deletion strains $\mathrm{K} 32$ was acetylated instead of modified to 5-aminopentanol. Furthermore, three genes ( $y a a O, y f k A$ and $y w / G$ ) were identified which influence the degree of EF-P K32 modification, however, their mode of action remains to be elucidated (Witzky et al., 2018). The following modification pathway was suggest: K32 becomes modified with hydroxypentanone, which in turns becomes dehydrate and forms pentanone, which is converted to 5 -aminopentanone by a hydroamination reaction, and reduced to 5-aminopentanol by Ymfl (Witzky et al., 2018). B. subtilis was reported to tune the activity of EF-P, although the mechanism is not yet fully understood. The deletion of EF-P in B. subtilis causes a reduced swarming motility, which is in line with the occurrence of poly(Pro) sequences in genes related to cell motility. The lack of a severe phenotype correlates with the general low abundance of poly(Pro) sequences in the B. subtilis genome. It was proposed that for bacteria with a high abundance of poly(Pro) sequences in their genome, EF-P is more important than in those with a low abundance of poly(Pro) sequences. Overall, the nature of many post-translational modifications of EF-P is yet not described. It still remains unknown whether other factors can perform similar task or compensate for the lack of EF-P. Organism with either no or two copies of EF-P have been reported, but the functional significance of these findings remains unclear (reviewed in: (Lassak et al., 2016)). For some gammaproteobacteria, such as E. coli or Vibrio cholera, an EFP-like protein (YeiP) was described as a paralog of EF-P (Richards et al., 2012). Whether it binds to the ribosome or can compensate for the lack of EF-P remains to be elucidated.

\subsubsection{EF-P interaction partners}

EF-P binds in an equimolar ratio to the ribosome. The binding site spans both subunits and is located between the P and E sites (Aoki et al., 2008; Blaha et al., 2009; Huter et al., 
2017). For most crystallographic studies, the ribosomes and EF-P used for crystallization were from different organisms and none of the complexes was stalled by a PPP sequence or contained a prolyl-nascent chain in the $\mathrm{P}$ site. However, a recent study combined cryo-electron microscopic structures with molecular dynamic simulations and biochemical insights on poly(Pro) stalled E. coli ribosomes containing post-translational modified E. coli EF-P (Huter et al., 2017).

In the structure, domain III of EF-P interacted with the $30 \mathrm{~S}$ subunit and mRNA, as well as with the P-site tRNA via the EF-P residues Y183 and R186 (Blaha et al., 2009). Replacing one of the residues with alanine (Y183A or R186A) caused the complete loss of EF-P function. Both residues are highly conserved across bacterial EF-P sequences (Huter et al., 2017). The conserved GDT motif in loop I (amino acids 144-146) in the C-terminal domain III is well resolved. EF-P was found to bind to the $\beta$-hairpin of S7 and cause a conformational change and shift of S7 by $7.4 \AA$ (Figure $8 \mathrm{~A}$ ). The shift positions the EF-P residues D145 and T146 in such a way that they could form hydrogen bonds with the mRNA E-site codon (Figure 8B). The potential significance of the mRNA E-site codon for EF-P binding was further strengthened by molecular dynamic simulations (MDS). A mechanism of mRNA E-site codon reading by EF-P was proposed, which is analogous to the stop codon reading by release factor 1 and 2 using SPF and PXT motifs (Huter et al., 2017; Zhou et al., 2012). Molecular dynamic simulations suggest that EF-P could sense the first two bases of the E-site codon and that the nature of the mRNA bases could influence the binding of EF-P. Upon accommodation of EF-P all purines bases would cause steric clashes (AAA and GGG codons) and hinder EF-P binding, whereas pyrimidine derivatives allow either less stable (UUU) or stable (CCX) accommodation of EF-P in the E site (Huter et al., 2017). This hypothesis is supported by the fact that proline codons contain pyrimidine bases in position 1 and 2: CCG (55\% codon-usage in E. coli), CCA (20\%), CCU (15\%) and CCC (10\%) (Maloy et al., 1996). The absence of domain III in the eukaryotic and archaeal EF-P homologue argues against the importance of the E-site interactions. On the other hand, elF5A was reported to be beneficial for translation independent of poly(Pro) stalling motifs (Pelechano and Alepuz, 2017; Schuller et al., 2017). The lack of domain III in elF5A may explain its broader substrate spectrum, while EF-P binding is dependent on the E-site codon, supporting the high context specificity of EF-P. This 
evidence suggests that the interactions of EF-P with the E site are important, but this has not been tested biochemically.

EF-P domain II interacts with the ribosomal protein L1 and is sandwiched between L1 domain I and II (Figure 8C) (Blaha et al., 2009; Huter et al., 2017). The L1 stalk is a highly dynamic structure which consists of 23S rRNA and the ribosomal protein L1 (Yusupov et al., 2001). Upon interacting with EF-P the L1 stalk adopts a closed conformation (Blaha et al., 2009). Such a closed conformation was previously reported for L1 interacting with a deacylated-tRNA in a P/E hybrid state, in which the exit of the E site is occupied by the L1 stalk (Chen et al., 2013; Cornish et al., 2009; Fu et al., 2011; Tourigny et al., 2013; Valle et al., 2003). The positively charged surface of L1 interacts with the negatively charged surface of EF-P, similarly to the L1-tRNA interaction. These interactions may help to release the deacylated tRNAs from the E site (Bock et al., 2013; Munro et al., 2010; Selmer et al., 2006; Yusupov et al., 2001). For elF5A a similar conformation was described, which is supported by the results of hydroxyl radical probing experiments (Gutierrez et al., 2013; Schmidt et al., 2016b). These findings suggest that L1 is important for EF-P binding. 

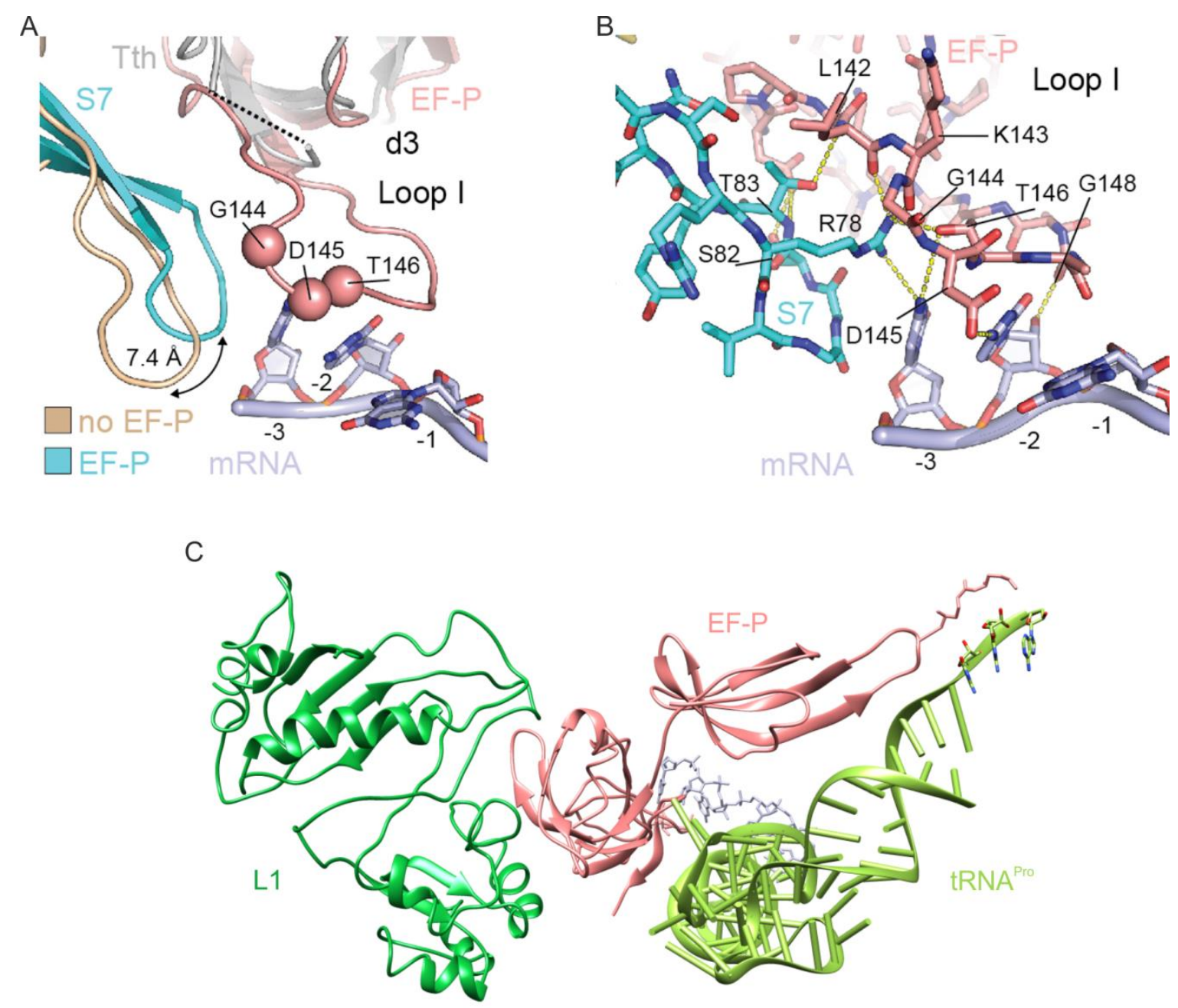

Figure 8: Interaction of EF-P with the mRNA, P-site tRNA and ribosomal protein L1.

A) EF-P-induced shift of the ribosomal protein $\mathrm{S7}$ (turquoise vs ochre) and the position of the EF-P domain III (d3) loop I. Residues G144, D145, and T146 of EF-P may interact with the E-site mRNA codon.

B) Potential hydrogen bonds between EF-P d3 loop I with S7 and with the mRNA. Hydrogen bonds are shown as yellow dashed lines.

C) Top view on the interaction of EF-P with the P-site tRNA and the ribosomal protein L1.

Figures $A$ and $B$ are modified from, and $C$ is based on [6ENU](Huter et al., 2017).

Domain II of EF-P interacts with the D-arm of the P-site tRNA. EF-P is sensitive to the geometry of the tRNA D-arm, determined by its stability and the lengths of the stem arm. Alternation of the D-arm while remaining the original tRNA ${ }^{\text {Pro }}$ body abolished the ability of EF-P to facilitate catalysis (Katoh et al., 2016). Interestingly, only tRNA ${ }^{\text {Pro }}$ isoacceptors and the initiator $\operatorname{tRNA}^{\mathrm{fMet}}$ share the same $\mathrm{D}$ arm geometry and are the only reported substrates of EF-P (Doerfel et al., 2013; Glick et al., 1979; Glick and Ganoza, 1975; Ude et al., 2013). In E. coli and T. thermophiles EF-P residue D69 interacts with U17a of the D-arm of the P-site tRNA (Blaha et al., 2009; Huter et al., 2017). In yeast no analogous interaction 
was reported (Melnikov et al., 2016; Schmidt et al., 2016b), which further supports the less defined substrate spectrum of elF5a.

Another important patch of EF-P is located within the positively charged region domain I, which provides an interaction hub with the 23S rRNA of the PTC, the acceptorstem and CCA-end of the P-site tRNA (Figure 8C) (Hanawa-Suetsugu et al., 2004; Huter et al., 2017). The domain I contains a conserved P-loop which is important for EF-Ps functionality. The key residue is K34 located in the P-loop of domain I. K34 is post-translationally modified by $R$-lysinylation and hydroxylation (Yanagisawa et al., 2010). Similar modifications of EF-P at homologous positons were reported for other bacterial EF-Ps, alF5A and elF5A (section: 1.3.4 and 1.3.7). Overall, the interactions of modified E. coli EF-P, unmodified T. thermophilus EF-P and hypusinylated elF5A from yeast with the CCA-end of the P-site peptidyl-tRNA are very similar (Figure 9).

A

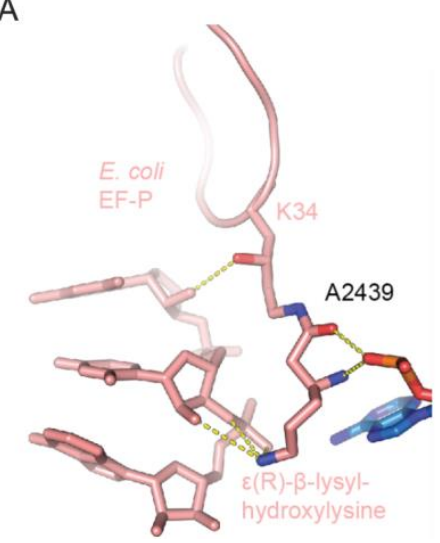

B

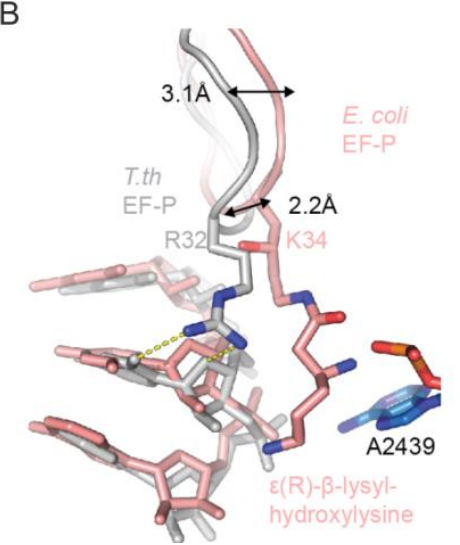

C

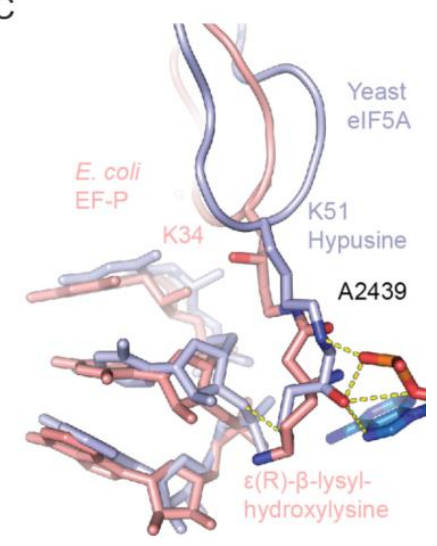

Figure 9: Comparison of E. coli EF-P and its homologues interacting with the P-site tRNA CCA-end.

A) E. coli EF-P bound to the ribosome. Potential hydrogen bonds between the posttranslationally added $\varepsilon(R)-\beta$-lysyl-hydroxylysine with the CCA-end of the peptidyl-tRNA are indicated as yellow dashed lines.

B) T. thermophiles EF-P bound to the ribosome superimposed on A. Different positioning of both EF-P molecules is indicated by the distance of the shift.

C) Yeast elF5A bound to the ribosome superimposed on $A$. The post-translational modifications of EF-P and elF5A extend into the PTC nearly to a similar degree. The conformation and resulting interaction are however distinctly different.

(Figure adapted from: (Huter et al., 2017))

The $\varepsilon(R)$ - $\beta$-lysylhydroxylysine 34 of $E$. coli extends deep into a crevice adjacent to the CCA-end. Its $\beta$-hydroxyl moiety is in hydrogen bonding distance with A76 and the $\varepsilon$-terminal $(R)$-lysyl amino moiety is in hydrogen bonding distance with the $2^{\prime} \mathrm{OH}$ of $\mathrm{C} 75 \mathrm{~s}$ ribose (Figure 9A). These backbone interactions stabilize the CCA-end and the P-site tRNA. In addition, an interaction between the post-translational modification of EF-P and the 
conserved nucleotide A2439 of the 235 rRNA was reported. For elF5A analogous interactions were proposed (Figure 9C). The unmodified T. thermophilus EF-P extends less deep into the PTC and only showed interactions with C75 (Figure 9B) (Huter et al., 2017).

The stabilization of the P-site peptidyl-tRNA by EF-P translates into the stabilization of the nascent chain. This conclusion was drawn from an increased density of the nascent chain during cryo-electron microscopy measurements. However, local resolution calculation on the nascent chain allowed only the modeling of four C-terminal residues, indicating that the rest of the nascent chain remains flexible. The two C-terminal prolines did not adopt an all-trans, nor an all-cis conformation. An all-trans conformation of Pro-Pro is found in di-prolyl-containing proteins, such as ribosomal protein S11 or L11, and in type II poly(Pro) helices (Fischer et al., 2015). Such conformation in a nascent-chain would cause steric clashes between the -2 residue of the nascent chain and nucleotide G2061 of the 23S rRNA. An all-cis di-prolyl conformation would drive the nascent-chain towards the A site and cause a steric clash with the ribosome. Instead, in the presence of modified EF-P Pro-Pro adopts an alternative trans-conformation. However, in order to obtain a detailed description of the diasteromer conformation, a better resolved structure is required. This unusual conformation allows the nascent chain to evade G2061 and to extend into the ribosomal exit tunnel. A similarly unusual Pro-Pro conformation was reported for the CMV-stalling peptidyl-tRNA (Matheisl et al., 2015). The overall position of the nascent chain corresponds to stalled peptidyl-tRNAs as found in SecM (Zhang et al., 2015), MifM (Sohmen et al., 2015) and VemP (Su et al., 2017). These insights suggest that the di-prolyl nascent chain cannot adopt its favored conformation, resulting in the induction of an energetically metastable conformation, which in turn causes the destabilization of the P-site peptidyl-tRNA and ultimately ribosomal stalling. Binding of EF-P, however, stabilizes the P-site peptidyl-tRNA and conducts the nascent chain into a conformation allowing peptide bond formation to occur and thereby restores translation.

\subsubsection{Eukaryotic homologue elF5A}

elF5A is a homolog of the bacterial EF-P. Similarly to EF-P, elF5A is post-translationally modified, but the modification is different. elF5A is uniquely post-translationally modified with the non-canonical amino acid hypusine (Cooper et al., 1983; Folk et al., 1980; Park et al., 1981, 1982). It's the post-translational modification is critical for the function of elF5A 
(Benne and Hershey, 1978; Gutierrez et al., 2013; Park et al., 2011; Park, 1989; Park et al., 1991; Saini et al., 2009; Schreier et al., 1977). In contrast to EF-P, unmodified elF5A stimulates the fM-Pmn reaction 10-fold, whereas modified elF5A enhanced the reaction by more than 100 -fold. Analogously to EF-P, elF5A alleviates poly(Pro) stalling. Interestingly, ribosome-profiling experiments revealed that in the absence of elF5A ribosomes were stalled not only at the proline runs, but also at non-poly(Pro) tri-peptide sequences. This indicates a broader functional context of elF5A in comparison to EF-P (Schuller et al., 2017). The ability to facilitate a variety of stalling-motifs might be explained by the lack of interactions between elF5A and the D-arm of the P-site peptidyl-tRNA or with the codon displayed in the E site. However, the role of elF5As is most likely to stabilize the CCA-end and mediate a peptide bond formation by inducing a favorable conformation of the nascent chain of the P-site peptidyl-tRNA (Gutierrez et al., 2013; Melnikov et al., 2016; Schmidt et al., 2016b). In contrast to EF-P, elF5a may have a more general role in elongation (Gregio et al., 2009; Henderson and Hershey, 2011; Saini et al., 2009; Schuller et al., 2017). Depleting elF5A in the cell caused an increased number of polysomes and increased ribosome transit times (Saini et al., 2009). Surprisingly, such an effect was not found in EF-P-depleted cells (Woolstenhulme et al., 2015). Recently, the impact of elF5A on termination was described. Termination by eRF1 was 16-fold enhances in the presence of elF5A, while such an effect was not described for EF-P (Schuller et al., 2017). The broad set of functions assigned to elF5A would require a high intra-cellular concentration and probably a high affinity for the ribosome. In fact, elF5A was reported to have roughly 273.000 copies per cell and hence, is equivalent abundant as the ribosomes. Therefore, elF5A is considered to be one of the most abundant proteins in yeast (Kulak et al., 2014; von der Haar, 2008). In E. coli 5,000 and 50,000 copies of the ribosome per cell, depending on the growth rate, were reported (reviewed in:(Bremer and Dennis, 2008)). For the elF5A analog EF-P 0.1 copies per ribosome were found (An et al., 1980), a later study suggests 20,000 copies per cell (Schmidt et al., 2016a). Hence, EF-P does not match the eiF5A-ribosome 1:1 ratio. For eIF5A an approximate dissociation constant of $9 \mathrm{nM}$ was determined (Rossi et al., 2016). For EF-P, however, binding determinants and the dissociation constant remain missing. 


\subsubsection{Post-translation modifications of e/alF5A}

elF5A is post-translationally modified by the non-proteinogenic amino acid hypsuine $\left(N^{\varepsilon}\right.$-(4-amino-2-hydroxybutyl)lysine) and is so far the only reported protein carrying this modification (Figure 10) (Cooper et al., 1983; Folk et al., 1980; Park et al., 1981, 1982). elF5A is modified immediately after emerging from the ribosome (Murphey and Gerner, 1987; Park, 1987). The modification is mediated by two specific enzymes, deoxyhypusine synthase (DHS) and deoxyhypusine hydroxylase (DOHH). DHS uses spermidine as substrate and transfers the 4-aminobutyl moiety onto the conserved residue K51 of yeast elF5A or K50 in human eIF5A (Cano et al., 2008; Park et al., 2010; Tong et al., 2009a; Wolff et al., 1990). The formation of deoxyhypusine via DHS relies on the coenzyme NAD ${ }^{+}$. The reaction proceeds through four steps, involving two imine intermediates as well as a hydride transition state (Wolff et al., 1997; Wolff et al., 1990; Wolff et al., 2000). DHS functions as a dimer, however, it tends to form a homotetramer to compensate for charges (Lee and Park, 2000; Wolff et al., 2000). DHS recognizes residues F30 to D80 of elF5A as recognition site for K51. Mutation of the target lysine or any amino acid exchange within the conserved P-loop of domain I abolishes the post-translational modification (Cano et al., 2008; Joe and Park, 1994; Park et al., 1998; Sasaki et al., 1996). The deoxyhypusine hydroxylase also uses the recognition site spanning residues $\mathrm{F} 30$ to D80, which is required for both, binding to and catalytic activation of the deoxyhypusine hydroxylase. As a substrate, DOHH preferentially recognizes the deoxyhypusinated lysine form, but also recognizes the unmodified lysine (Kang et al., 2007; Park et al., 2006; Thompson et al., 2003). In contrast to DHS, DOHH is only essential in higher eukaryotes (Patel et al., 2009; Sasaki et al., 1996; Sievert et al., 2014). The DOHH of the corresponding gene, LIA1, in fission and budding yeast caused only light growth defects (Sievert et al., 2014; Weir and Yaffe, 2004). In line with these findings, both recombinant deoxyhypusinated and hypusinated elF5As were able to facilitate the $\mathrm{fM}-\mathrm{Pmn}$ reaction to a similar extend (Park et al., 2011). In higher eukaryotes, the knock-out of DOHH caused not only the expected lack of the hypusine modification, but also abolished the preceding deoxyhypusination (Sievert et al., 2014). An in vitro elF5A modification assay revealed that the deoxyhypusine modification indeed was reversible (Park et al., 2003). It was proposed that the transformation of deoxyhypusine to hypusine masks the deoxyhypusine modification and removes it from the substrate-product pool of DHS and 
thereby prevents the backward reaction to an unmodified elF5A. elF5A can be additionally modified by acetylation and phosphorylation at different modification sites (Ishfaq et al., 2012; Kang et al., 1993; Klier et al., 1993). The acetylation and deacetylation of elF5A was linked to its intracellular location (Ishfaq et al., 2012). The role of the phosphorylation of elF5A S102 remains unknown (Kang et al., 1993; Klier et al., 1993).

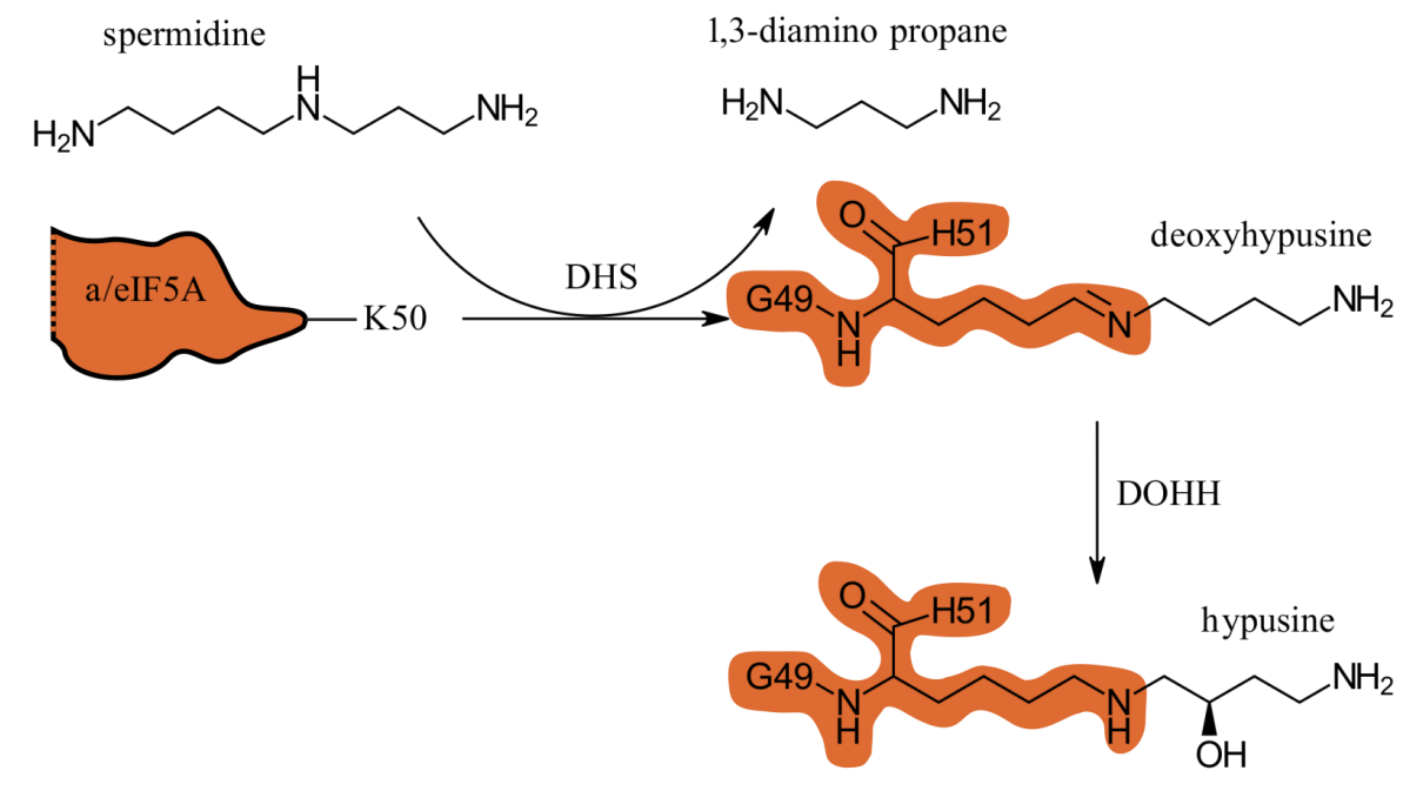

Figure 10: Post-translational modification of elF5A.

A) Archaeal and eukaryotic IF5A modified to hypusine at K50 by sequential action of DHS and DOHH.

The archaeal elF5A homolog, alF5A, can be either hypusinated, deoxyhypusinated or in very few cases both modification were found (Bartig et al., 1990). In archaea the mechanism of post-translation modification is not yet fully understood. For DHS a homolog could be identified, which appeared to mainly rely on spermidine as substrate and NAD ${ }^{+}$as co-factor (Bartig et al., 1992; Bassani et al., 2018). For DOHH no homolog has been identified yet (Park, 2006; Wolff et al., 2007). In some higher eukaryote, a DHS was described to be bifunctional, performing the classic DHS-reaction as well as the DOHH catalyzed dehydration reaction (Quintas-Granados et al., 2016). For the studied archaea, however, such bifunctionality of DHS was not observed (Bassani et al., 2018). However, archaeal DHS can have a broader substrate pool. For a desoxyhypusine modified alF5A, it was reported that the DHS used agmatine as substrate, which in a second reaction became transformed into deoxyhypusine (Prunetti et al., 2016). The usage of an alternative substrate is likely to result from the varying intracellular polyamine 
composition in archaeal sub-species, as out of 117 halophilic archaeal strains only 20 contained traces of spermidine (Hamana et al., 2009). Because agmatine serves as the precursor for an essential modification of tRNA ${ }^{\text {lle }}$ it is omnipresent in archaea (Blaby et al., 2010; Ikeuchi et al., 2010; Mandal et al., 2010).

\subsection{Scope of the thesis}

Elongation factor $\mathrm{P}$ is required to alleviate poly(Pro)-stalled ribosome complexes, ensuring the continuation of translation. Although the catalytic mechanism is well described, it is not known whether EF-P binding is limited to poly(Pro)-stalled complexes or occurs at every complex with a vacant E site. We developed a FRET based assay using fluorescent ribosome complexes and a quencher labeled EF-P, to study the binding of EF-P to different ribosome complexes. Biochemical and structural data suggested the D-loop of the tRNA bound in the $P$ site, the post-translational modification of EF-P and the ribosomal protein L1 as potential key interaction partners of EF-P. In order to dissect their potential contribution to either binding or catalysis, we constructed different functional EF-P variants and used ribosomes lacking L1 in the developed binding and activity assays. Overall, this study contributes to the understanding of EF-P binding to the ribosome and how EF-P ensures the smooth translation of poly(Pro) runs. 


\section{Results}

\subsection{EF-P binding assay}

In order to study the binding of EF-P to the ribosome, a Förster resonance energy transfer (FRET)-based assay was developed. FRET describes the energy transfer between two nearby chromophores, in which the initially excited "donor" chromophore non-radiatively transfers energy via dipole-dipole coupling to a second "acceptor" chromophore. Following energy transfer, the excited electrons of the acceptor dye relax and, depending on the nature of the acceptor, this relaxation can result in the emission of a photon at a longer wavelength than the light used to excite the donor, and also the quenching of the donor fluorescence. Due to the nature of the dipole coupling, the FRET efficiency depends on the distance between the two dyes $(r)$ according to $1 / r^{6}$ (described in: (Harris, 2010; Helms, 2008; Valeur, 2012)). This strong distance-dependence makes FRET highly suitable for the study of binding and conformational changes between two fluorescently-labeled reactants. The distance at which the FRET efficiency reaches $50 \%$ is called Förster distance $\left(R_{0}\right)$. Depending on the distance between the FRET partners, the assay will be either be more sensitive (for $\mathrm{R}$ close to $\mathrm{R}_{0}$ ) or less sensitive to small changes in the dye spacing. For the EF-P binding assay developed in this work, a FRET-efficiency of about $95 \%$ can be assumed for the bound state, and thus the assay will report robustly on binding while being insensitive to other events such as conformational rearrangement. The combination of FRET with a rapid kinetic method, such as stopped flow, then allows real time monitoring of binding.

To study EF-P binding to the ribosome, EF-P was labeled with a fluorescence acceptor and the ribosome with a fluorescence donor (Figure 12A). The mRNA, tRNAs, ribosomal protein L1 and L33 were considered as potential labeling sides for the fluorescent donor. Structural studies suggested that ribosomal protein L33 might be the most suitable labeling site to form a FRET pair with bound EF-P (Blaha et al., 2009). L33 is located between the $E$ and $P$ site on the 50S subunit, and in contrast to the other considered labeling sides, it does not change the position during translation. Upon binding of EF-P to the E site, EF-P and L33 come into close proximity, which is a prerequisite for FRET. Preparation of ribosome complexes containing an Alexa Fluor ${ }^{\mathrm{TM}} 488$-labeled L33 has been were already established, and their biochemical and photophysical properties validated 
by Belardinelli et al. (Belardinelli and Rodnina, 2017; Belardinelli et al., 2016a; Belardinelli et al., 2016b; Goyal et al., 2015; Sharma et al., 2016). As fluorescent acceptor attached to EF-P, the non-fluorescent quencher QSY35 was used. In contrast to a classic FRET pair, the increasing concentration of the fluorescent acceptor does not affect the fluorescence signal and hence requires no correction, e.g. during titrations.

\subsubsection{Labeling of EF-P}

In order to monitor EF-P binding, EF-P was labeled with a fluorescence acceptor. Sulfhydryl-reactive fluorescent dyes allow the site-specific labeling at cysteine residues. Wild type EF-P contains one cysteine, which, however, is not solvent accessible and did not react with dye. For the site-specific labeling of EF-P, single amino acid residues were exchanged to cysteine. Candidate amino acids were selected considering potential steric clashes with the ribosome, degree of conservation of the exchanged amino acid residue, distance to the FRET partner and potential solvent accessibility based on the X-ray structure of EF-P-bound to the ribosome [PDB 4V6A] (Blaha et al., 2009). Ten potential mutation sites were identified (Figure 12 and Table 1). Each EF-P variant was overexpressed together with the EF-P modifying enzymes in E. coli BL21 (DE3). EF-P was purified by metal affinity chromatography via an N-terminal 6xHis-tag followed by a gel-filtration chromatography. 


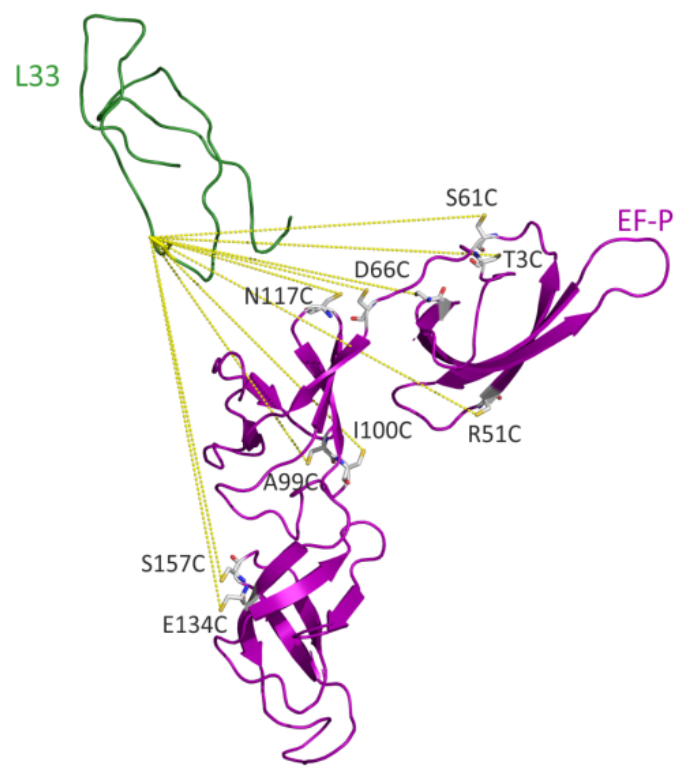

Table 1: EF-P labeling site and distance $(r)$ to L33 P31C

\begin{tabular}{ll}
\hline Labeling site & $r(\AA ̊ 丿)$ \\
\hline T3C & 45 \\
M16C & 36 \\
R51C & 46 \\
S61C & 41 \\
D66C & 27 \\
A99C & 37 \\
I100C & 40 \\
N119C & 27 \\
E134C & 46 \\
S157C & 44 \\
*Distances based on X-ray structure [PDB 4V6A].
\end{tabular}

Figure 11: Structure and labeling sites of L33 and EF-P.

Indicated are the introduced cysteine residues for site-specific labeling of EF-P (magenta) and the labeling position in L33 (green). Distances are shown as yellow dashed lines for each potential FRET pair. Based on X-ray structure [PDB4V6A (Blaha et al., 2009)].

To achieve a high labeling efficiency and prevent dimerization by disulfide-bridges, EF-P cysteine residues were reduced prior to labeling. The $\mathrm{pH}$ was adjusted such that the sulfhydryl groups remained deprotonated, while the thio-reactive linker of the dye remained active. Four different fluorescent acceptors (ATTO540Q, QSY-7, QSY-9 and QSY-35) with either iodacetamide or maleimide as reactive group were tested. With three of the tested dyes, independent of the linker, only low labeling efficiencies were achieved or the labeled protein precipitated. Maleimide would have been the linker of choice, because it is more stable and has a higher specificity. Added in large excess, iodoacetamide was reported to be able to alkylate amines (lysine), carboxylates (aspartate, glutamate), imidazols (histidine) and thioester (methionine) and therefore is less specific (Jullien and Garel, 1981; Michaelis, 1934; Yang and Attygalle, 2007). The only dye which yielded a high labeling efficiency of $80 \%$ and a soluble protein was QSY35, which was only available as iodoacetamide.

The QSY35-labeled EF-P variants were applied to a stopped-flow apparatus to test their ability to quench the fluorescent of ICs containing 50 S subunits which were reconstituted with L33 labeled with AlexaFluor ${ }^{\text {TM }} 488$ (L33Alx). For most tested variants only a small change in donor fluorescence (10 - 15\%) was observed, with the exception of 
EF-P D66C (QSY35) (EF-P(QSY)), which caused a 30\% fluorescence decrease upon binding to the ribosome. This EF-P derivative was used in the following study.

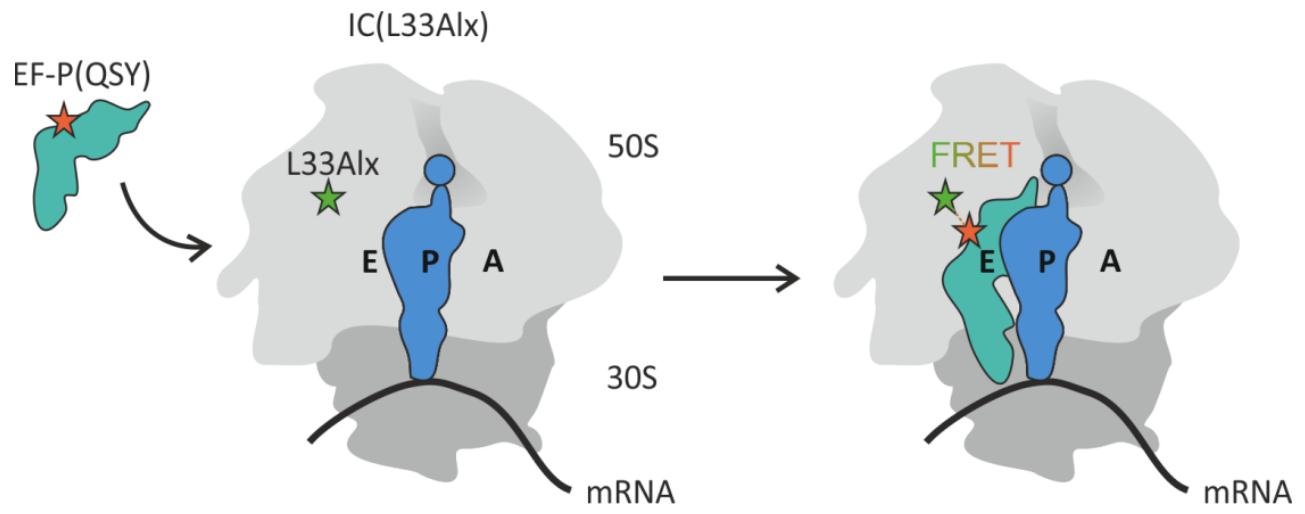

Figure 12: EF-P binding assay.

A) Schematic of the EF-P binding assay based on FRET between EF-P(QSY35) (turquoise, the position of fluorescence reporter is indicated orange and ribosomes (IC(L33Alx)) containing L33 labeled with AlexaFluor ${ }^{\text {TM }} 488$ (L33Alx) (green). The P-site tRNA is shown in blue.

\subsubsection{Validation of the EF-P binding assay}

We next validated that the observed fluoresc3nce donor change reflects reversible binding of EF-P to the ribosome. The amplitude of the fluorescence change of $70 \mathrm{SIC}(\mathrm{L33Alx}$ ) was roughly $30 \%$ in the presence of EF-P(QSY). In the absence of EF-P(QSY), the signal of the donor remained stable over time (Figure 13A). The eukaryotic EF-P homolog, eIF5A, was reported to interact with the 60S subunit (Rossi et al., 2016), which prompted us to test whether EF-P(QSY) binds to 50S(L33Alx) subunit. However, no signal change was observed, suggesting that EF-P does not bind to the $50 \mathrm{~S}$ subunits. A small (10\%) fluorescence change was observed with vacant 70S ribosomes containing L33Alx, suggesting that EF-P interacts weaker with vacant ribosomes than with ICs (10\% vs $30 \%$ change in amplitude).

To further validate the assay and to obtain insights into the binding kinetics, we recorded time courses of EF-P(QSY) binding to $70 \mathrm{IC}($ L33Alx) (Figure 13B). A concentration dependent fluorescence decrease was observed, supporting the notion that the observed event indeed depends on EF-P binding to the ribosome. The apparent rate constants of EF-P binding to ICs were determined by two-exponential fitting with an additional slope. The apparent rate constants (Figure 13C) and the respective amplitudes (Figure 13D) were used to calculate the association $\left(k_{\text {on }}\right)$ and dissociation $\left(k_{\text {off }}\right)$ rate constants and the 
equilibrium dissociation constant $\left(K_{d}\right)$. The $K_{d}$ is defined as the quotient of the dissociation and association rate constants. The apparent rate constant of the fast step $\left(k_{a p p 1}\right)$ depends linearly on the concentration, indicating that it reports on the binding step. The $k_{\text {on }}$ value can be derived from the slope of the linear fit of the concentration dependence. The intercept of the fit with the $y$-axis yields the $k_{\text {off. }}$ The association rate constant was $\mathrm{k}_{\mathrm{on}}=56 \mu \mathrm{M}^{-1} \mathrm{~s}^{-1}$ and dissociation rate $\mathrm{k}_{\text {off }}=58 \mathrm{~s}^{-1}$. The binding rate constant $\left(\mathrm{K}_{\mathrm{d}}\right)$ was about $1 \mu \mathrm{M}$ based on the $k_{\text {on }}$ and $k_{\text {off }}$ values. In addition, the $K_{d}$ value can be obtained from the concentration dependence of the amplitude of the signal change (reviewed in: (Bernasconi, 1976; Klostermeier, 2017)), which was about $1 \mu \mathrm{M}$ (Fig. 12D). The slower phase of the binding time course had a very small amplitude (Fig. 12D) and no concentration dependence (Fig. 12C) and was not considered further.

In order to investigate whether the labeling affected EF-P function, its ability to facilitate peptide bond formation was tested. Fluorescence-labeled fMP post-translocation complexes (Post(fMP)(L33Alx) were mixed with puromycin (Pmn) acting as A-site substrate analog in the presence or absence of EF-P and EF-P(QSY). The amino moiety of the aminonucleoside antibiotic puromycin reacts with peptidyl-tRNA, resulting in a peptide-puromycin product. The reaction of Post(fMP)s with Pmn is accelerated by EF-P up to 90-fold (Doerfel et al., 2013), providing a large dynamic window to detect changes. The reaction of Post(fMP)(L33Alx) with Pmn was performed in a quench-flow apparatus which is suitable to measure rapid kinetics in a millisecond to second range. The products were purified from unreacted substrates via reversed-phase chromatography and the yield plotted as function of the reaction time. The time courses were similar with EF-P and EF-P(QSY). The time courses were analyzed by one-exponential fitting (Figure 13E), allowing to derive an observed reaction rate. The reaction rate was $0.2 \pm 0.1 \mathrm{~s}^{-1}$ in the absence of EF-P, $4.2 \pm 0.8 \mathrm{~s}^{-1}$ in the presence of native EF-P, and $9.4 \pm 0.8 \mathrm{~s}^{-1}$ with $E F-P(Q S Y)$. Thus, fluorescence labeling did not impair the functional activity of the ribosome complex or EF-P. 
A

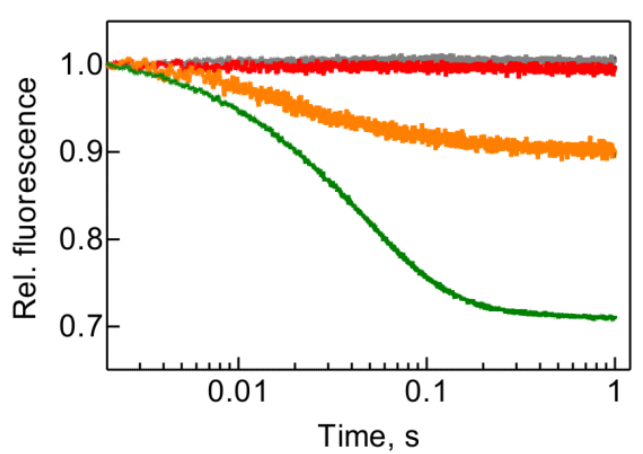

C

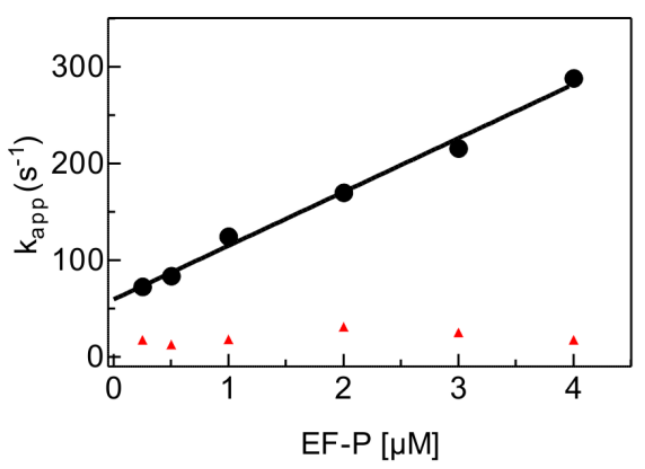

E

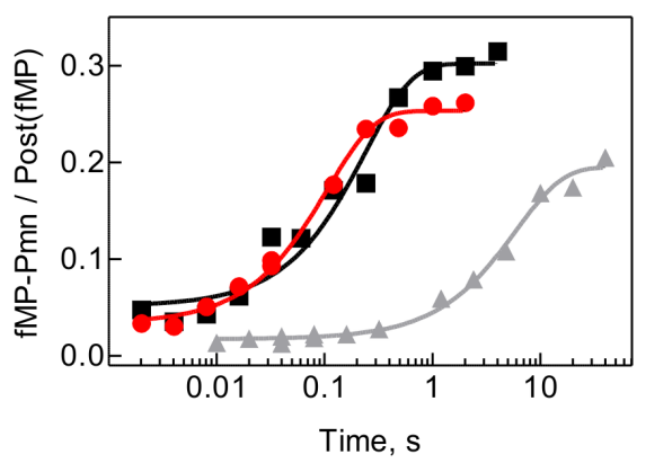

B

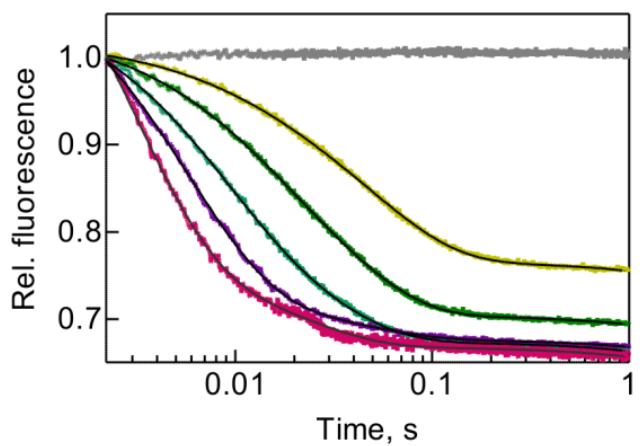

D

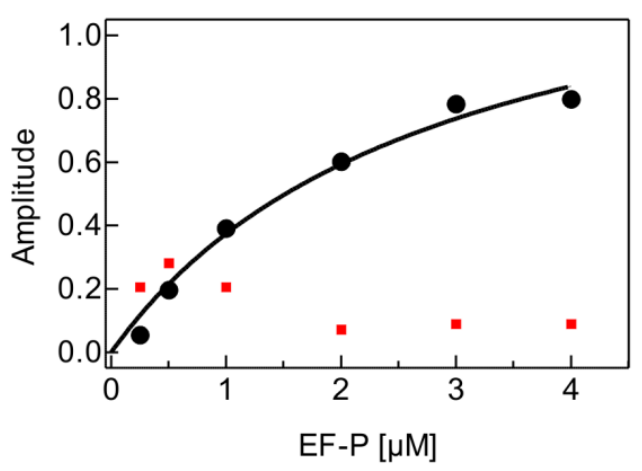

$\mathrm{F}$

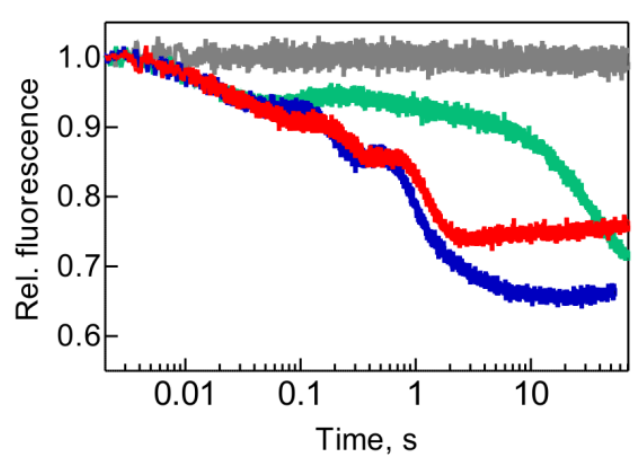

Figure 13: Validation of the EF-P binding assay.

A) Interaction of EF-P with potential binding partners, monitored by FRET using a stopped-flow apparatus. Fluorescent L33Alx-labeled 50S subunits (red), vacant 70S ribosomes (orange) and $70 S$ IC (green) were rapidly mixed with EF-P(QSY). Buffer was used in the donor-only trace (grey).

B) Binding of EF-P(QSY) to unpurified 70S IC(L33Alx). The binding was monitored in a stoppedflow apparatus and ICs were titrated with EF-P(QSY) at the following concentrations: $0.25 \mu \mathrm{M}$ (ochre), $0.5 \mu \mathrm{M}$ (green), $1 \mu \mathrm{M}$ (turquoise), $3 \mu \mathrm{M}$ (purple) and $4 \mu \mathrm{M}$ (magenta). The fits are shown as light color of corresponding trace. Buffer was used for the donor-only trace (grey).

C) Concentration dependence of the apparent rate constants ( $k_{\text {app1 }}$, black and $k_{a p p 2}$, red) derived from a two-exponential fit of the traces in $B$ and the linear fit of $k_{\text {app1 }}$.

D) Concentration dependence of amplitudes (black, $A_{\text {app1 }}$ and red, $A_{\text {app } 2}$ ) derived from a two-exponential fit of the traces in B. Black line shows a hyperbolic fit of $A_{1}$ value.

E) Activity of fluorescence-labeled components. Formation of fMP-Pmn was measured using Post(fMP)(L33Alx) complex $(0.2 \mu \mathrm{M})$ and Pmn $(10 \mathrm{mM})$ in the absence (grey) or presence of EF-P(QSY) $(3 \mu \mathrm{M}$; red) and unlabeled EF-P ( $3 \mu \mathrm{M}$; black). The experiments was carried out using a quench-flow apparatus, were reactants were rapidly mixed, incubated for the indicated times, then quenched and the products analysed by HPLC.

F) Time resolved in vitro translation of fMPPPF monitored by FRET. 70S IC(L33Alx) was rapidly 
mixed in a stopped-flow apparatus with EF-P(QSY) $(0.5 \mu \mathrm{M})$, EF-G $(1 \mu \mathrm{M})$, Pro-TC $(2 \mu \mathrm{M})$ and Phe-TC $(2 \mu \mathrm{M})$. Red: in the presence of all components, blue: lacking Phe-TC; and mint: lacking EF-G. Buffer was used for the donor-only trace (grey).

In order to study the binding of EF-P to the ribosome during translation, EF-P(QSY35) was added to a fully reconstituted translation assay. Here, IC(L33Alx) programmed with an mRNA encoding FMPPPF were used as a model complex, as translation of the PPP motif requires EF-P (Doerfel, 2016; Ude et al., 2013). However, the observed time courses were too complex to analyze by exponential fitting (Figure 13F). In the absence of EF-G, a small initial decrease in fluorescence was followed by a very slow, biphasic fluorescence decrease. The origin of these fluorescence changes is not clear. In the absence of EF-G, binding of the first TC-Pro should occur, resulting in the formation of the hybrid state of the ribosome, but the subsequent translocation cannot take place. EF-P seems not to bind to the hybrid-rotated ribosomes, as the initial amplitude change is small, but with time EF-P binding to the fraction of the non-rotated ribosomes may shift the equilibrium towards ribosome-EF-P complexes, resulting in a further fluorescence change; some extent of spontaneous translocation cannot be excluded as well. The time course obtained in the absence of Phe-TC, showed a small fluorescence decrease similar to the initial decrease recorded in the absence of EF-G, followed by another small decrease, a plateau and a final large decrease in fluorescence. In a simplest model, this patter would reflect the binding of the TC-Pro, translocation, and binding of the $2^{\text {nd }}$ TC-Pro, which reflect rounds of EF-P binding and dissociation from different transient translation steps. After the incorporation of the $2^{\text {nd }}$ Pro, stable binding of the $3^{\text {rd }}$ TC-Pro is impaired (Doerfel et al., 2013; Peil et al., 2013; Ude et al., 2013), and this complex is the true substrate for EF-P binding, which explains the largest fluorescence change. Addition of TC-Phe reduces the final fluorescence, as a fraction of EF-P dissociates from the ribosome upon TC-Phe incorporation and translocation. Despite the set of different conditions, the traces were too complex to assign specific translation events to each signal change. Nevertheless, overall a robust FRET-based EF-P binding assay was developed, while its components remained biochemically active. 


\subsection{Determinants for EF-P}

Previous reports suggested that the E-site mRNA codon, the P-site peptidyl-tRNA, post-translational modification of EF-P, and the ribosomal protein L1 may affect the binding of EF-P to the ribosome (Blaha et al., 2009; Doerfel et al., 2013; Huter et al., 2017; Katoh et al., 2016). So far, all conclusions regarding the binding of EF-P have either been indirectly derived from the ability of EF-P to catalysis peptide bond formation or concluded from structural insights. By applying the established EF-P binding assay, the impact of the described binding determinants was tested.

\subsubsection{E-site context}

A cryo-electron microscopic study suggested a potential interaction between EF-P and the E-site codon (Huter et al., 2017). It was proposed that in the ribosome-EF-P complex the bases of the E-site codon are hydrogen bonding distance with the conserved residues D145, T146 and G148 in loop I of EF-P domain III. This finding was further supported by molecular dynamics simulations which predicted that the nature of the bases of the E-site codon can influence binding. Biochemical experiments showed mutations in loop I of EF-P domain III reduced or abolished the ability of EF-P to rescue poly(Pro)-stalled ribosomes (Huter et al., 2017). In order to address a potential interaction the loop I with the mRNA E-site codon, the corresponding amino acids in EF-P were mutated in the EF-P D66C labeling variant. The conserved aspartic acid residue (D145) was exchanged to valine, and the conserved threonine (T146) to alanine and valine. In addition, EF-P truncation ( $\Delta$ L142-G144, D145G, T146G, $\Delta$ A147-T149) variant was constructed. Residue G148 was not changed, because it is supposed to interact with the mRNA via its amino acid backbone. The exchange of glycine with proline would have changed the putative backbone interaction, however, it would also have a major influence on the conformation of the loop. EF-P variants were overexpressed in E. coli BL21 (DE3), purified according to standard procedure and labeled with QSY35.

The labeled EF-P variants were used for the EF-P binding assay (Figure 14A). The fluorescence signal was small with any of the EF-P loop I mutants than with EF-P(QSY). Both T146 variants showed an amplitude of less than 5\% and EF-P D145V of 20\%, compared to $30 \%$ amplitude change upon binding of EF-P(QSY). The reduced fluorescence 
decrease could indicate a lower affinity of EF-P or correspond to a lower FRET efficiency, resulting from a different orientation of the bound EF-P.
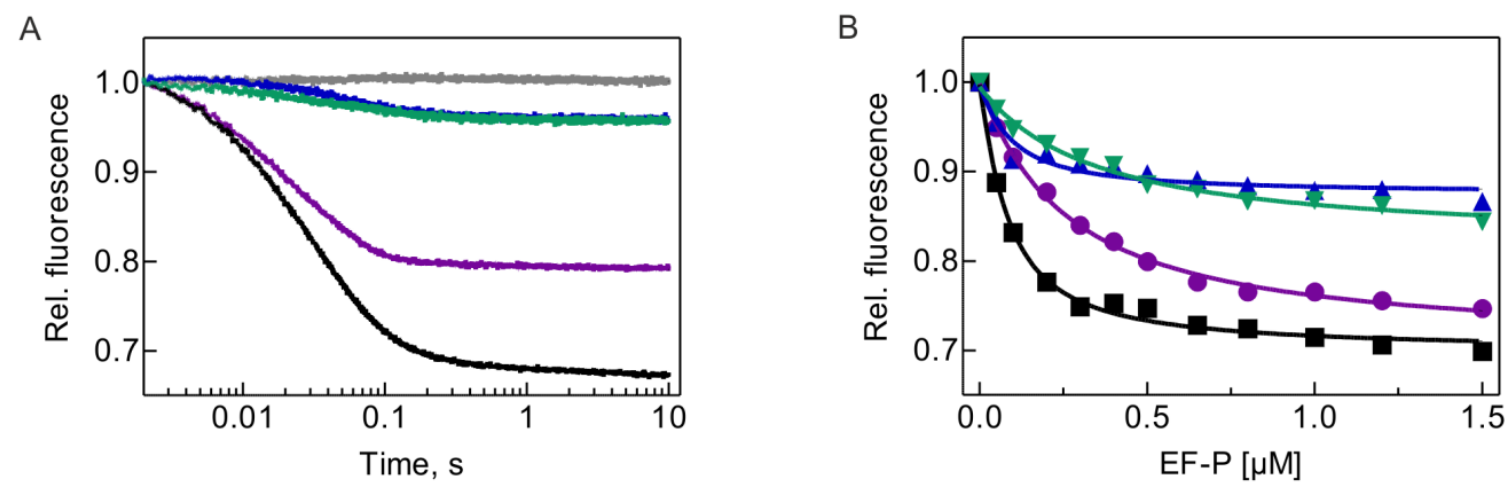

Figure 14: Binding of EF-P flexible-loop variants.

\begin{abstract}
A) Binding of EF-P(QSY) domain III variants to 70S IC(L33Alx). EF-P was rapidly mixed with 70S IC(L33AIX) in a stopped-flow apparatus und FRET was monitored. Traces in the presence of $\mathrm{EF}-\mathrm{P}(\mathrm{QSY})$ are shown in black, T146A(QSY) in blue, T146V(QSY) in green, and D145V(QSY) in purple. Buffer was used for the donor-only trace (grey).

B) Titration of EF-P(QSY) domain III variants to 70S IC(L33Alx) in a fluorimeter. The fluorescence change with increasing concentration of EF-P(QSY) variants in equilibrium was plotted. The color code is as in A. The dissociation constant $K_{d}$ for each EF-P variant was calculated using a quadratic equation.
\end{abstract}

In order to determine the $K_{d}$ value for the EF-P variants, an equilibrium titration was performed (Figure 14B) in a spectrofluorimeter monitoring the change of the fluorescence upon addition of increasing concentrations of EF-P. The observed amplitudes are very similar to the one observed in the corresponding stopped-flow experiment. To analyze the titration curve, we used a quadratic equation, which takes into account the difference between added and free concentration of the titrated component (EF-P in our case) depending on the concentration of the ligand present in constant concentration (the ribosome complex). The $K_{d}$ value was $0.2 \mu \mathrm{M}$ for EF-P(QSY) and $0.1-0.3 \mu \mathrm{M}$ for the EF-P domain III loop I variants (D145V: $0.1 \mu \mathrm{M}$, T146A: $0.1 \mu \mathrm{M}$ and T146V: $0.3 \mu \mathrm{M}$ ). Thus, against the expectation, the loop I mutants did not impair the binding affinity of EF-P. This finding indicates that the E-site codon does not affect EF-P binding and speaks against the sampling of the displayed codon by loop I of EF-P domain III. However, the applied method did not allow to determine the binding and dissociation rate. For that a rapid kinetic technique, such as stopped-flow, would be required.

Because the EF-P loop variants did not show an increased dissociation constant, we asked the question whether the native loop is able to distinguish different bases. 
Molecular dynamics simulations suggested that the nature of the -2 and -3 base can hinder, weaken or allow binding of EF-P (Huter et al., 2017). To study the interaction of EF-P (with its native loop I) with the E-site, complexes were needed which vary only in the codon displayed E site. To change the E-site codon, while keeping the same peptidyl-tRNA in the P site, we designed mRNA constructs that share the same sequence, except for the codon downstream of the AUG initiation codon (Figure 15). Initiation complexes were formed using these mRNAs, with either an Ile (IleIC) or Pro (ProlC) codon in the E site. Thus, the interaction of EF-P with the E-site codon could be study, while other determinants of EF-P binding, in particular the tRNA in the P site, remained the same.

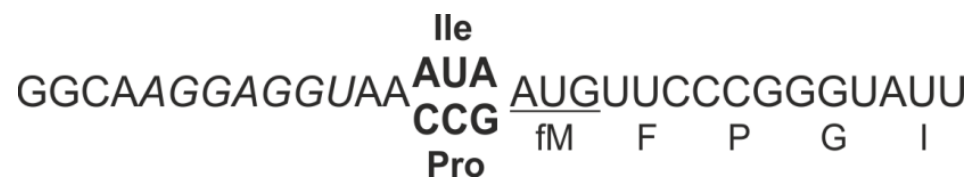

Figure 15: mRNA constructs used to study the EF-P E-site mRNA codon interaction.

mRNAs have the same sequence except for the codon down preceding the AUG. The isoleucine codon (AUA) was suggested to be unfavorable for EF-P binding, whereas, the proline codon (CCG) represents a suggested favorable binding interface (Huter et al., 2017). The ShineDalgarno sequence is indicated in italics.

Fluorescence-labeled initiation complexes with either of these mRNAs were formed and used in the EF-P binding assay. The change in fluorescence was dependent on EF-P concentration (Figure 16A and B). The apparent rate of EF-P binding was determined by two-exponential fitting with an additional slope. The binding rate $\mathrm{k}_{\text {on }}$ and the dissociation rate were determined from the linear fit of the $k_{a p p 1}$ concentration dependence (Figure 16C and D, Table 2). 
A

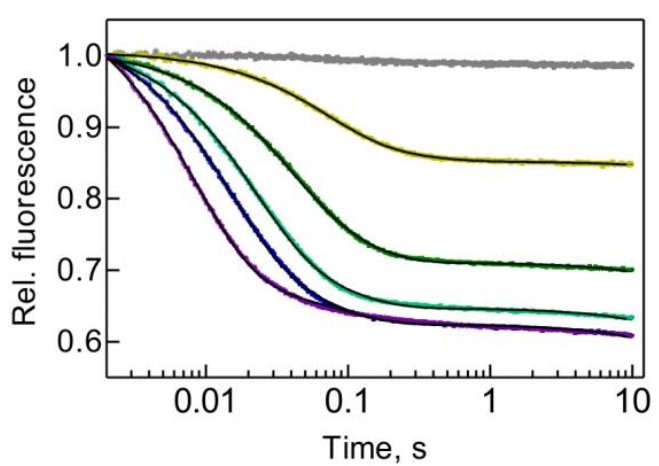

B

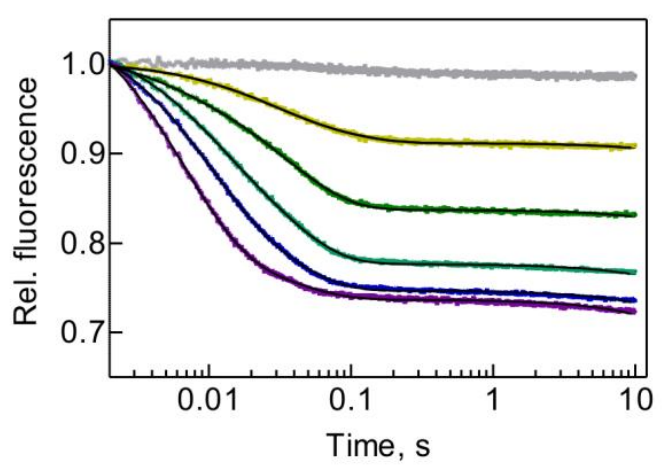

C

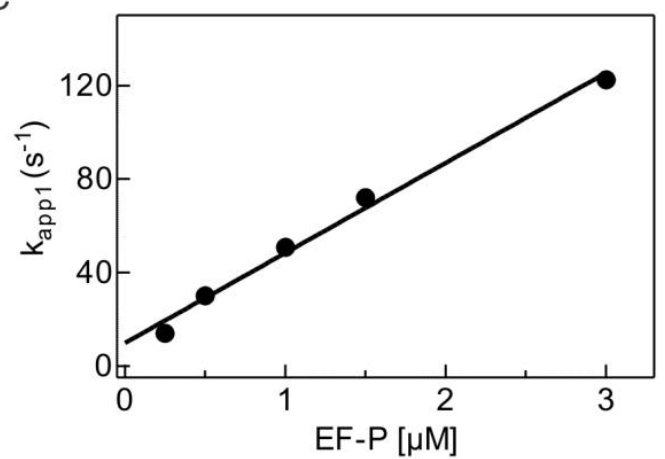

D

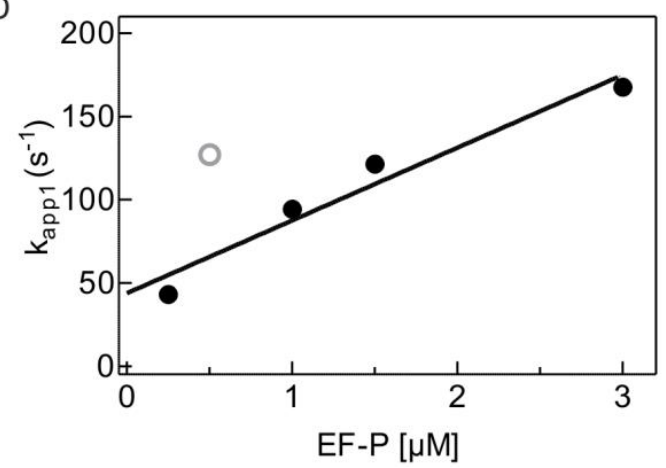

Figure 16: Influence of the E-site codon on EF-P binding.

A) Binding of EF-P(QSY) to initiation complexes displaying the lle codon AUA in the E site. The binding was monitored in a stopped-flow apparatus. Ile IC(L33AIX) mixed with increasing EF-P(QSY)concentrations: $0.25 \mu \mathrm{M}$ (ochre), $0.5 \mu \mathrm{M}$ (green), $1 \mu \mathrm{M}$ (turquoise), $1.5 \mu \mathrm{M}$ (blue) and $3 \mu \mathrm{M}$ (purple). The fits are shown as light color of corresponding trace. Buffer was used for the donor-only trace (grey).

B) Same as A, but with the Pro codon CCG in the E site.

C) Concentration dependence and linear fit of the apparent rate constant $\left(k_{a p p 1}\right)$ derived from a two-exponential fit of the traces in A.

D) Same as in C with an outlier in grey, for traces of B.

Table 2: Rates derived from EF-P interaction with ICs displaying different E-site codons.

\begin{tabular}{lllll}
\hline & $\mathrm{k}_{\text {on }}\left[\mu \mathrm{M}^{-1} * \mathrm{~s}^{-1}\right]$ & $\mathrm{k}_{\text {off }}\left[\mathrm{s}^{-1}\right]$ & $\mathrm{K}_{\mathrm{d}}[\mu \mathrm{M}]$ & $\mathrm{K}_{\mathrm{d}} *[\mu \mathrm{M}]$ \\
\hline IleIC(L33Alx) & $39 \pm 2$ & $10 \pm 2$ & $0.3 \pm 0.1$ & $0.25 \pm 0.02$ \\
ProlC(L33Alx) & $44 \pm 7$ & $44 \pm 12$ & $1 \pm 1$ & $0.14 \pm 0.02$
\end{tabular}

$\mathrm{K}_{\mathrm{d}}{ }^{*}$ is derived from titration in a fluorimeter. Whereas, $\mathrm{k}_{\text {on }}$ and $\mathrm{k}_{\text {off }}$ are derived from stopped-flow experiments. The $K_{d}$ is the quotient of $k_{\text {off }}$ and $k_{\text {on }}$.

Surprisingly, EF-P binding rate constants were similar on the two codons (Table 2). The dissociation rate for EF-P and complexes with a proline codon in the E site is 
erroneous due to an outlier. However, the in equilibrium titration showed the association rate constants to be independent of the displayed E-site codon.

To further validate the conclusion that the E-site codon has little effect on EF-P binding, we performed equilibrium titrations of initiation complexes displaying the CCG codon in the E-site (ProIC(L33Alx)) with the EF-P variants with mutations in domain III loop I. Binding of EF-P(QSY) and D145V(QSY) had a maximum amplitude of about $30 \%$ and the titrations were nearly identical. The amplitude for T146A(QSY) and T146V(QSY) is only 5\%, and these two titration curves also coincide. The observed amplitudes are similar to those of EF-P binding to IleIC(L33Alx) (Figure 16). For all tested variants including EF-P $(w t)$ a $\mathrm{K}_{d}$ of $0.15 \mu \mathrm{M}$ was calculated (Figure 17B). Thus, the affinity of EF-P to the initiation complex is independent of the E-site codon or the loop I in EF-P domain III. Overall, this work suggests that integrity of the flexible loop I is of relevance for the activity of EF-P, the nature of the E-site codon, however, is of little relevance for EF-P binding and activity.
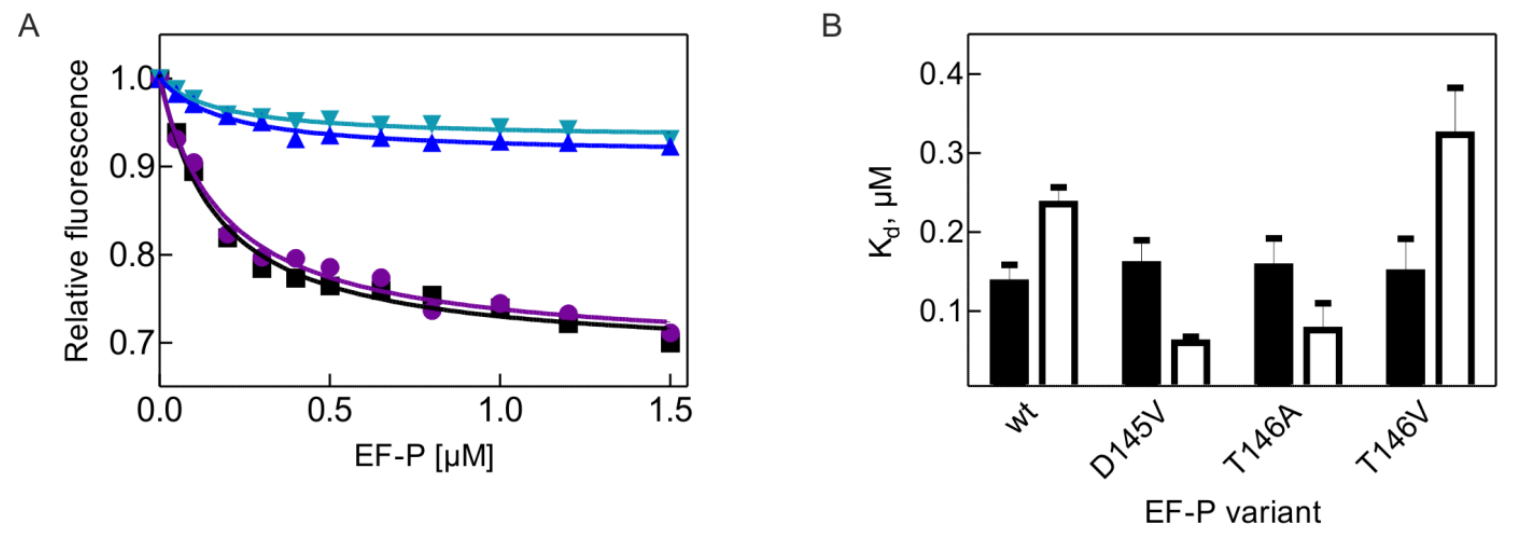

Figure 17: Binding and affinity of EF-P flexible loop variants to different E-site codons.

\begin{abstract}
A) Titration of EF-P(QSY) domain III variants to IC(L33Alx) in a fluorimeter. Data points corresponding to EF-P(QSY) are shown in black, T146A(QSY) in blue, T146V(QSY) in green and $\mathrm{D} 145 \mathrm{~V}(\mathrm{QSY}$ ) in purple. Buffer was used for the donor-only trace (grey). The dissociation constant $\mathrm{K}_{\mathrm{d}}$ for each EF-P variant was calculated using a quadratic equation.

B) Equlibrium dissociation constants $\left(K_{d}\right)$ of EF-P(QSY) and its domain III variants for IC(L33Alx) displaying either the Pro codon CCG (black) or the Ile codon AUA (white) in the E site. The $K_{d} s$ values are from $A$.
\end{abstract}

\title{
2.2.2 Peptidyl-tRNA context
}

Structural studies showed an interaction between EF-P and the D-arm of the P-site bound peptidyl-tRNA (Blaha et al., 2009; Huter et al., 2017). The relevance of the D-arm for EF-Ps function was demonstrated by biochemical studies (Katoh et al., 2016). The D-arm architecture of tRNA ${ }^{\text {Pro }}$, which is shared by tRNA ${ }^{\text {fMet }}$ but not other tRNAs, was shown to be 
a perquisite for the ability of EF-P to facilitate peptide bond formation. This effect was proposed to result from a higher affinity of EF-P for the D-arm of tRNA ${ }^{\text {Pro }}$ and tRNA ${ }^{\text {fMet }}$ compared to other tRNAs (Katoh et al., 2017a).

In chapter $2.1 \mathrm{I}$ have shown that EF-P binds to the initiation complexes displaying fMet-tRNA $^{\text {fMet }}$ as a P-site tRNA. In order to address the question whether and to which extend the P-site peptidyl-tRNA influences the binding of EF-P, different fluorescent post-translocation complexes were prepared and EF-P binding was measured (Figure 18A). The complexes Post(fMF)(L33Alx), Post(fMP)(L33Alx) and Post(fMPP)(L33Alx) were formed and titrated with EF-P(QSY). Fluorescence changes and amplitudes were similar for the three complexes (Figure 18A, B and C). The binding rate constant $k_{\text {on }}$ and the dissociation rate constant $k_{\text {off }}$ were determined from the linear fit of the $k_{a p p 1}$ concentration dependence (Figure 18D, E and F). The binding and dissociation constants were used to calculate the equilibrium dissociation constants $\left(K_{d}\right)$ for each complex (Table 3). 
A

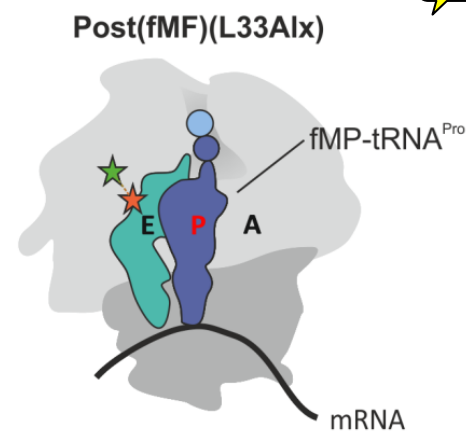

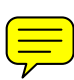

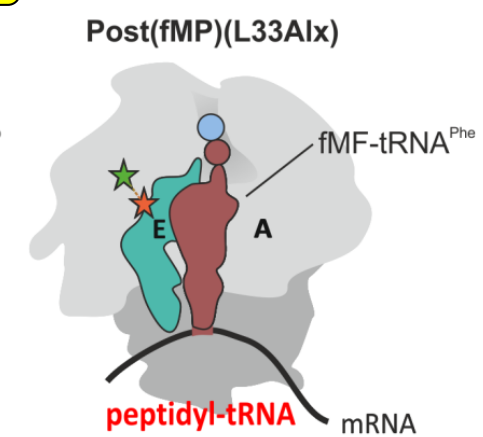

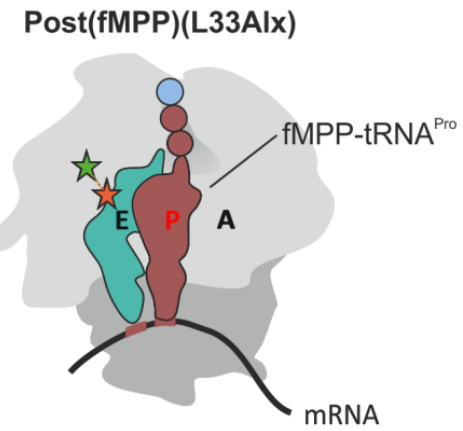

B

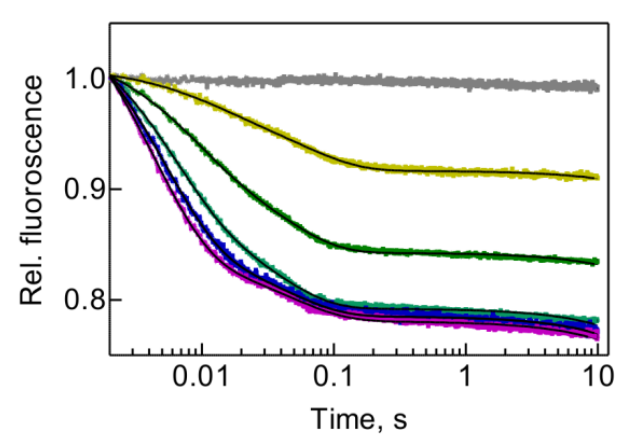

C

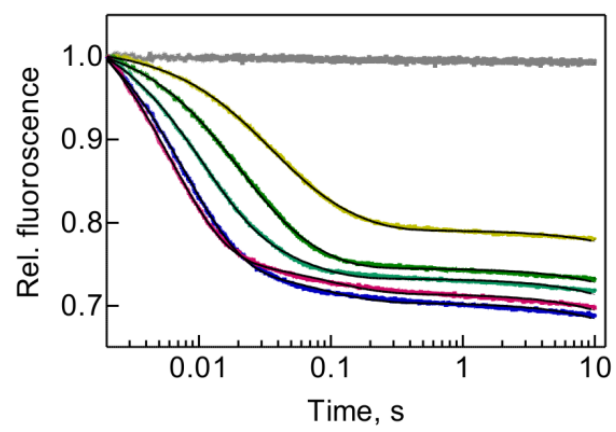

D

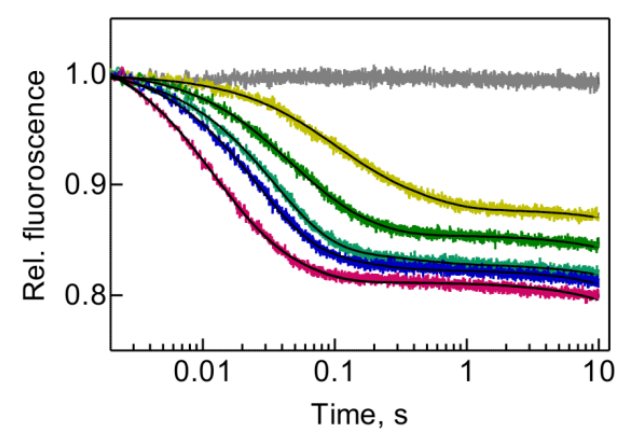

E
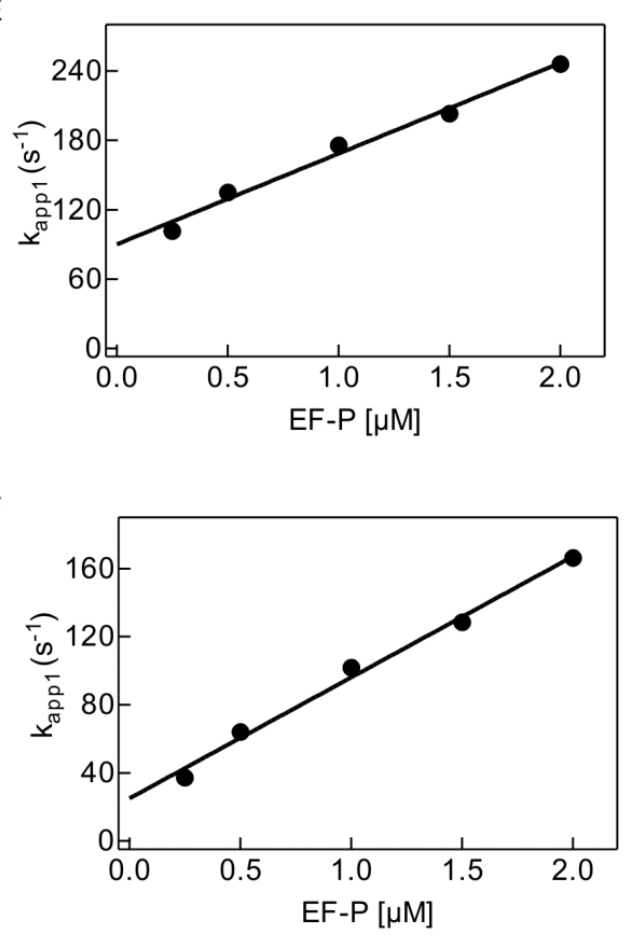

G

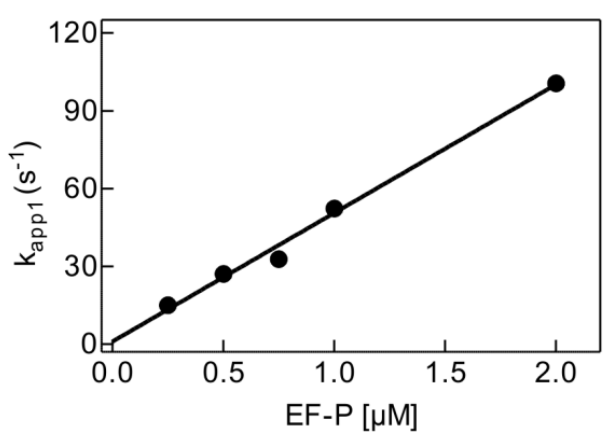

Figure 18: Influence of the P-site tRNA on EF-P binding.

A) Schematic of the EF-P binding assay to study the interaction of EF-P with peptidyl-tRNA.

B) Binding of EF-P(QSY) to fMF-post-translocations complexes (Post(fMF)(L33Alx)) monitored in a stopped-flow apparatus. Post(fMF (L33Alx) was mixed with different EF-P(QSY) concentrations: $0.25 \mu \mathrm{M}$ (ochre), $0.5 \mu \mathrm{M}$ (green), $1 \mu \mathrm{M}$ (turquoise), $1.5 \mu \mathrm{M}$ (blue) and $2 \mu \mathrm{M}$ (magenta). Buffer was used for the donor-only trace (grey).

C) As B, but with Post(fMP)(L33Alx) complexes which contain tRNA ${ }^{\text {Pro }}$.

D) As B, but with Post $(\mathrm{fMPP})(\mathrm{L} 33 \mathrm{Al} \mid \mathrm{x})$ complexes with tRNA ${ }^{\text {Pro }}$ in the $\mathrm{P}$ site and a Pro codon displayed in the $\mathrm{E}$ site.

E) Concentration dependence and linear fit of the apparent rate constant $\left(k_{\text {app } 1}\right)$ derived from a two-exponential fit of the traces in B. 
F) Same as in E, for traces of $C$.

G) Same as in E, for traces of D.

Table 3: Rates of the EF-P interaction with post-translocation complexes.

\begin{tabular}{llll}
\hline Post $(X)$ & $k_{\text {on }}\left[\mu \mathrm{M}^{-1} *^{-1}\right]$ & $\mathrm{k}_{\text {off }}\left[\mathrm{s}^{-1}\right]$ & $\mathrm{K}_{\mathrm{d}}[\mu \mathrm{M}]$ \\
\hline $\mathrm{fMF}$ & $79 \pm 5$ & $90 \pm 6$ & $1.1 \pm 0.1$ \\
$\mathrm{fMP}$ & $71 \pm 4$ & $25 \pm 4$ & $0.4 \pm 0.1$ \\
$\mathrm{fMPP}$ & $50 \pm 3$ & $1 \pm 3$ & $0.02 \pm 0.06$ \\
\hline
\end{tabular}

The rates of EF-P binding to Post(fMF)(L33Alx) and Post(fMP)(L33Alx) were found to be the same, whereas for Post(fMPP)(L33Alx) the binding rate was 1.5-fold slower. Comparing complexes with a functional context (proline containing) with non-proline containing complexes, EF-P dissociated from Post(fMF)(L33Alx) 3- to 90-fold faster, with an affinity reduced by 3 - to 50 -fold (Table 3 ). Comparing the two proline containing complexes, showed that the addition of one proline to the nascent chain, resulted in a slight reduction of the on rate and but in a 25 -fold reduction of the off rate. Hence, the diprolyl moiety itself or a resulting alternative positioning of the peptidyl-tRNA has to explain the differences in binding. Overall, these findings are in agreement with the biological relevance of EF-P. Complexes, potentially requiring EF-P assistance during translation were bound with a higher affinity. The lower $K_{d}$ results from a reduced $k_{\text {off, }}$ while $k_{\text {on }}$ remained roughly the same. This finding suggesting a sampling mechanism of EF-P based on a $k_{\text {off }}$ effect. Overall, the findings indicate that the P-site peptidyl-tRNA has an impact on the EF-P binding kinetic, however, the functional context of a poly(Pro)-stalled complex must provide additional interaction surfaces, resulting in a reduced dissociation and hence, higher affinity.

\subsubsection{EF-P modification}

Unmodified EF-P is impaired in its ability to facilitate peptide-bond formation between consecutive Pro residues and was proposed to have a lower affinity towards the ribosome (Doerfel et al., 2013; Ude et al., 2013). In order to investigate the impact of the modification on binding, the genes of the modifying enzymes EpmA, $\mathrm{B}$ and $\mathrm{C}$ were deleted 
from the overexpression plasmid encoding the EF-P labeling variant. Overexpression of EF-P leads to a dramatic increase of its cellular concentration. Previous studies have shown by mass-spectrometry that natively expressed EF-P modification enzymes are not sufficient to sufficiently modify the overexpressed EF-P and the majority of EF-P remained unmodified (Doerfel et al., 2013). The binding of unmodified EF-P(QSY) was tested in the EF-P binding assay using the following post-translocation complexes: Post(fMF)(L33Alx), Post(fMP)(L33Alx) and Post(fMPP)(L33Alx). For all three complexes a concentrationdependent decrease of the fluorescent signal was observed (Figure 19A, B and C). The $k_{\text {on }}$ and $k_{\text {off }}$ were determined from the linear fit of the apparent rate constant $k_{a p p 1}$ concentration dependence (Figure 19D, E and F) and used to calculate the equilibrium dissociation constants $\left(K_{d}\right)$ for each complex (Table 4). 
A

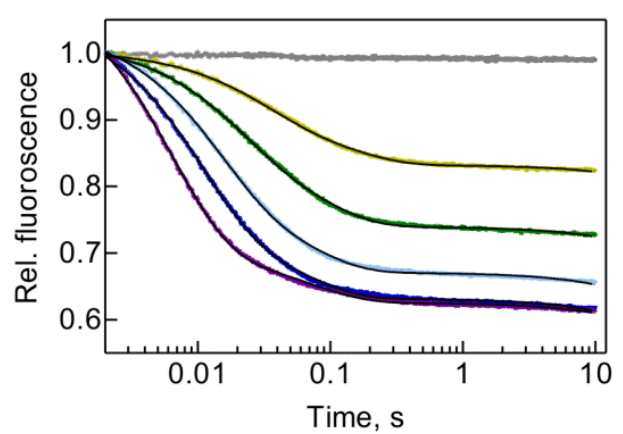

B

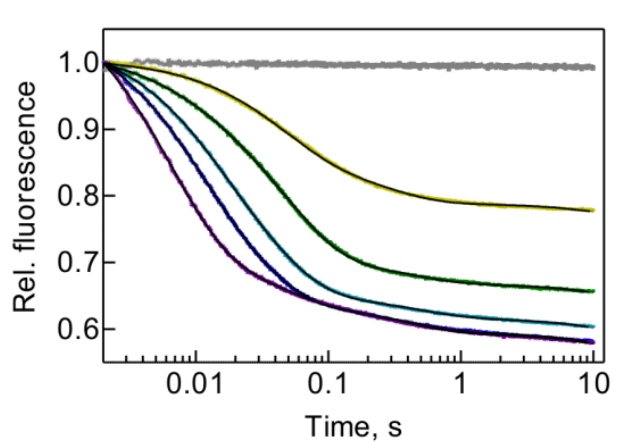

C

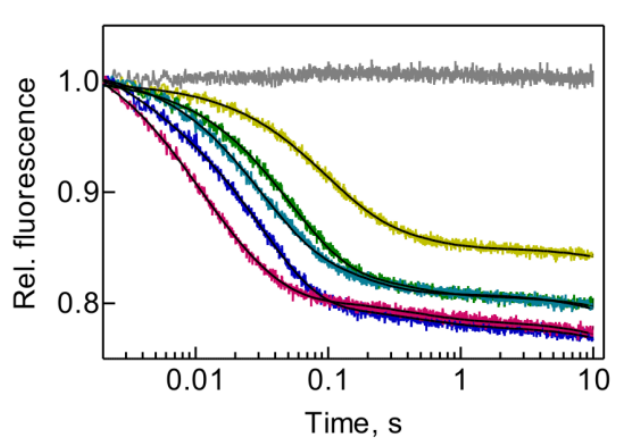

D

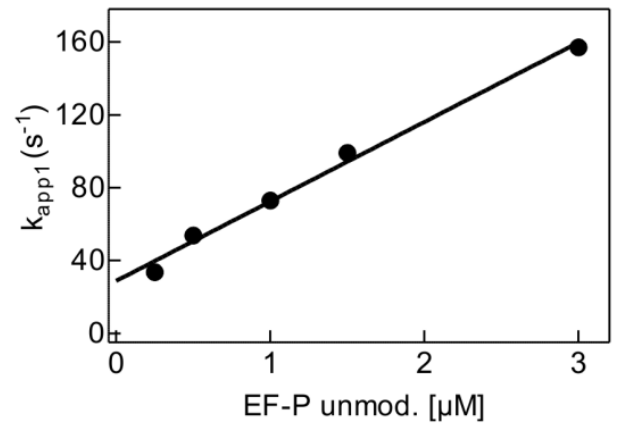

E

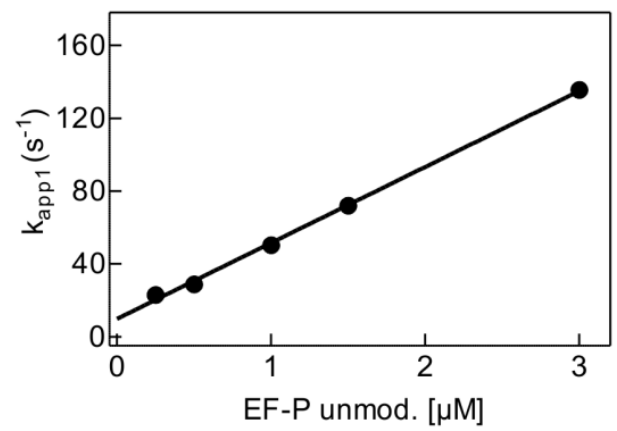

F

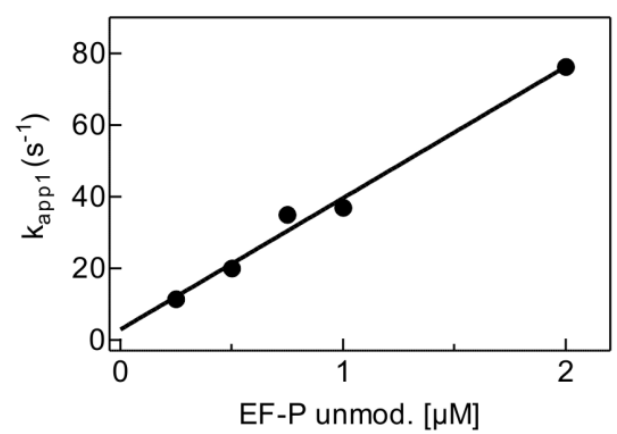

Figure 19: Effect of the ( $\beta$ )-hydroxy-lysinylation on EF-P binding.

A) Binding of unmodified EF-P(QSY) to fMF-post-translocations complexes (Post(fMF)(L33Alx)). The binding was monitored in a stopped-flow apparatus. Post(fMF (L33Alx) were titrated with EF-P(QSY): $0.25 \mu \mathrm{M}$ (ochre), $0.5 \mu \mathrm{M}$ (green), $1 \mu \mathrm{M}$ (turquoise), $1.5 \mu \mathrm{M}$ (blue) and $3 \mu \mathrm{M}$ (purple). Buffer was used for the donor-only trace (grey).

B) As A, but with Post(fMP)(L33Alx) complexes.

C) As A, but with Post(fMPP)(L33Alx) complexes and additionally $2 \mu \mathrm{M}$ EF-P(QSY) (magenta).

D) Concentration dependence and linear fit of the apparent rate constants $\left(k_{a p p 1}\right)$ derived from a two-exponential fit of the traces in A.

E) Same as in D, for traces of B.

F) Same as in D, for traces of C. 
Table 4: Rates of unmodified EF-P interacting with post-translocation complexes.

\begin{tabular}{llll}
\hline Post $(X)$ & $k_{\text {on }}\left[\mu M^{-1} * s^{-1}\right]$ & $k_{\text {off }}\left[s^{-1}\right]$ & $K_{D}[\mu M]$ \\
\hline fMF & $44 \pm 2$ & $29 \pm 3$ & $0.7 \pm 0.1$ \\
fMP & $42 \pm 1$ & $10 \pm 1$ & $0.2 \pm 0.1$ \\
fMPP & $37 \pm 2$ & $3 \pm 2$ & $0.08 \pm 0.07$ \\
\hline
\end{tabular}

The association rate constants of unmodified EF-P were similar for the tested post-translocation complexes, in-line with the results obtained with fully-modified EF-P. The dissociation rate constants of unmodified EF-P showed the same tendencies as with modified EF-P. The dissociation of unmodified EF-P from fMF-complexes was 3- to 10-fold faster than from proline-containing complexes. A comparison of the two proline containing complexes showed that the addition of one proline to the nascent chain results in a 3-fold reduction of the off rate. Interestingly, modified EF-P showed a 25-fold difference between POST(fMP) and POST(fMPP) complexes. Overall, unmodified EF-P binds all complexes with the same rate, whereas the dissociation varies depending on the complex. This suggests that despite its catalytic relevance the modification of EF-P also contributes to binding. However, the observed difference in binding between modified and non-modified EF-P are too little to account fully for the reduced activity of unmodified EF-P.

\subsubsection{Ribosomal protein L1}

EF-P bound to the ribosomes was reported to interact with the ribosomal protein L1 which was proposed to be involved in EF-P binding and dissociation (Blaha et al., 2009; Huter et al., 2017). The EF-P binding assay employing FRET between EF-P and the ribosomal protein L33 was not suitable to study the role of the ribosomal protein L1 for EF-P binding, because this would have required a $50 \mathrm{~S}$ subunit variant lacking both $\mathrm{L} 1$ and L33 (Cornish et al., 2009). To create such a double deletion variant is not feasible. To overcome this bottleneck, EF-P D66C was labeled with Alexa Fluor ${ }^{\mathrm{TM}} 488$ (EF-P(Alx)), which 
allowing to use a change in the anisotropy upon binding of EF-P to $\Delta \mathrm{L} 150 \mathrm{~S}$ subunits (Trabuco et al., 2010). Increasing concentrations of initiation complexes lacking L1 $(I C(\Delta L 1))$ added to EF-P(Alx) and the change in fluorescence anisotropy of the EF-P(Alx) was monitored (Figure 20A).

A

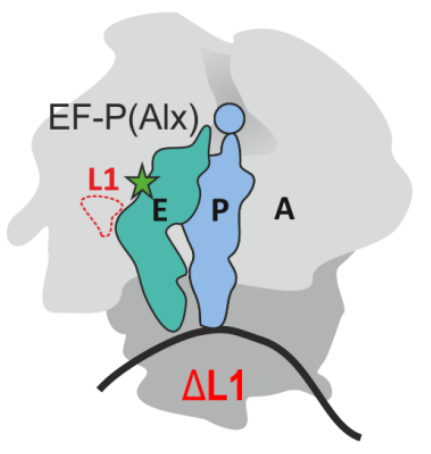

B

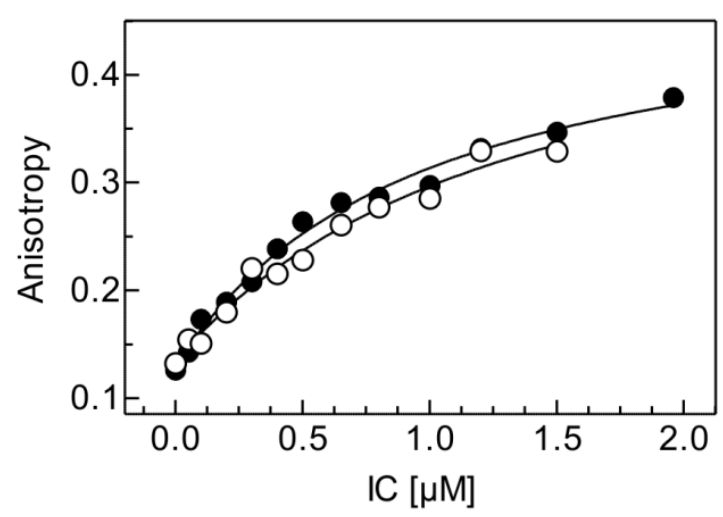

Figure 20: EF-P binding in the absence of L1.

A) Schematic of EF-P(Alx) binding to $I C(\Delta L 1)$. The binding was studied by monitoring the anisotropy change of EF-P(Alx).

B) Change of EF-P(Alx) anisotropy with increasing concentration of $70 \mathrm{~S} \mathrm{IC,} \mathrm{fitted} \mathrm{with} \mathrm{the}$ quadratic equation; closed symbols, $70 \mathrm{SIC}(w t)$; open symbols, IC( $\Delta \mathrm{L} 1)$.

The sample was exposed to linearly polarized light, in which the electric field vector oscillates in a single defined plane. Randomly polarized light can be converted into linearly polarized light using a polarizer. However, it was found that excited fluorophores emit partially polarized light and that the degree of polarization increased with the size of the dye and viscosity of the solvent, while an increase in the temperature decreased the degree of polarization. This means that at a defined temperature the fluorescence anisotropy correlates with the mobility of the dye. Thus, a less mobile emitting species will emit light which is more polarized. Hence, freely rotating fluorophores in solution have exhibit a low anisotropy, whereas fluorophores associated with large molecules have a higher anisotropy. The anisotropy values were plotted as a function of the IC concentration. For both complexes the same anisotropy change with increasing ribosome complexes was observed (Figure 20B). The $\mathrm{K}_{d}$ values derived by fitting to a quadratic equation were $1.0 \pm 0.2 \mu \mathrm{M}$ for native IC and $1.3 \pm 0.4 \mu \mathrm{M}$ for $\Delta \mathrm{L} 1 \mathrm{IC}$. Thus, $\mathrm{L} 1$ does not influence the affinity of EF-P to the ribosome. However, no information on the binding and dissociation rates were obtained, which would require further rapid kinetic 
techniques and chase experiments. Overall, we could show that the affinity of EF-P to the ribosome is independent of the ribosomal protein L1.

\subsection{Catalysis of peptide bond formation by EF-P}

So far we found that the nature of the P-site peptidyl-tRNA had a major effect on the binding kinetics of EF-P (section: 2.2.2), while the other suggested binding determinants had no significant contribution to the binding. The affinity of EF-P to initiation complexes was neither effect by the displayed codon in the E site, nor by the lack of ribosomal protein L1 (section: $2.2 .1 \&$ 2.2.4). The post-translational modification of EF-P showed a minor effect on the binding of EF-P and its lack resulted in slightly reduced association rate and increased dissociation rate (section: 2.2.3). The observed differences in binding appear to be too little to account for the reported reduced catalytic activity of unmodified EF-P (Doerfel et al., 2013; Ude et al., 2013). Because most of the suggested EF-P key interaction partners did not contribute to binding, different assays were used to study their influence on the ability of EF-P to facilitate peptide-bond formation.

\subsubsection{The E-site codon}

Structural data suggest that both sidechains and the backbone of the conserved GDT motif in loop I of domain III of EF-P come into hydrogen-bridging distance with the E-site codon (Huter et al., 2017). Hence, the nature of the E-site codon and the residues of the conserved loop could alter the interaction of EF-P with the ribosome.

To test this experimentally, EF-P activity was tested with different ribosome complexes in a quench-flow apparatus using the puromycin assay. The reaction between initiation complexes and the A-site substrate analog puromycin was shown to be facilitated by EF-P more than 5-fold (Doerfel et al., 2013), which makes the reaction suitable to test the reactivity of EF-P. Initiation complexes displaying either the isoleucine codon AUA or the proline codon CCG in the E-site were treated with the A site substrate analog puromycin in the presence of sub-saturating concentrations of EF-P (Figure 21A). The time courses of $\mathrm{fMet}-\mathrm{Pmn}$ formation were independent of the E-site codon (Figure 21B). The reaction rate was calculated by one-exponential fitting of the time courses. In both cases, the reaction rate was the same $\left(k_{o b s}=0.43 \pm 0.04 s^{-1}\right)$. This shows that the E-site codon does not affect the ability of EF-P to stimulate peptide bond formation. The relevance of loop I of 
domain III of EF-P was addressed in collaboration with the Wilson lab, which established different loop I variants. In order to address the suggested interaction of GDT sidechain with the E-site codon, the corresponding amino acids were substituted with alanine (EF-P $\left.{ }_{144} A A A_{146}\right)$. The contribution of the GDT backbone to a potential E-site interaction was investigated by deleting 1, 2 or 4 residues of loop I (EF-P (loopl $\Delta 1$ ), (loopl $\Delta 2$ ), and $($ loopl $\Delta 4))$. The eukaryotic EF-P homolog elF5A consists only of two domains. To mimic this protein, an elF5A analog EF-P variant missing the whole domain III was created (EF-P ( $\Delta$ domain III). These variants were tested for their ability to stimulate the synthesis of a fMPPPF peptide in a reconstituted in vitro translation system (Figure $21 \mathrm{C}$ ). In the translation reactions supplemented with EF-P (loopI $\Delta 1$ ), 70\% of all complexes produced the fMPPPF product. In the presence of EF-P (144AAA $\left.{ }_{146}\right)$ or EF-P (loopI $\left.\Delta 2\right), 50 \%$ and $40 \%$ of the complexes formed products. With EF-P (loopI $\Delta 4)$ and EF-P ( $\Delta$ domain III), almost no product formation was observed. Because the translation reaction competes with side reactions, such as peptidyl-tRNA drop-off, the product yield directly correlates with the activity of EF-P. Impaired EF-P catalyzes less product formation in a given time, while the drop-off reaction proceeds at constant rate, reducing the available substrates and hence limits the number of possible translation reactions. The maximum product yield was determined by supplementing the translation mix with EF-P (wt), in which $70 \%$ of the complexes formed the full-length product, which is in agreement with previously reported data (Doerfel et al., 2013). EF-P (wt) activity was only achieved by EF-P (loop I $\Delta 1)$. The deletion of the adjacent residue in loop I, EF-P (loopl $\Delta 2)$, resulted in a reduced activity. The deletion of the flexible loop, resulted the inactivation of EF-P (loopl $\Delta 4)$. Complete activity loss was also observed for EF-P lacking domain III. Interestingly, the replacement of the GDT motif that was proposed to interact with the E-site codon with AAA (EF-P ${ }_{144} \mathrm{AAA}_{146}$ ) caused only a slight reduction in activity. To further investigate the role of the D145 and T146, EF-P (D145L), (D145V), (T146A) and (T146V) variants were created and tested in the puromycin assay (Figure 21D and E). 
A

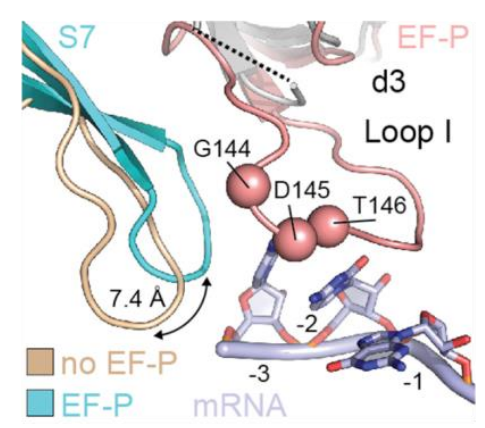

C

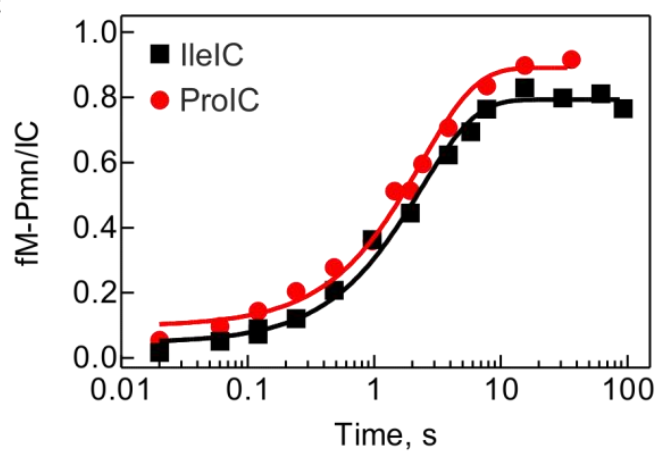

E

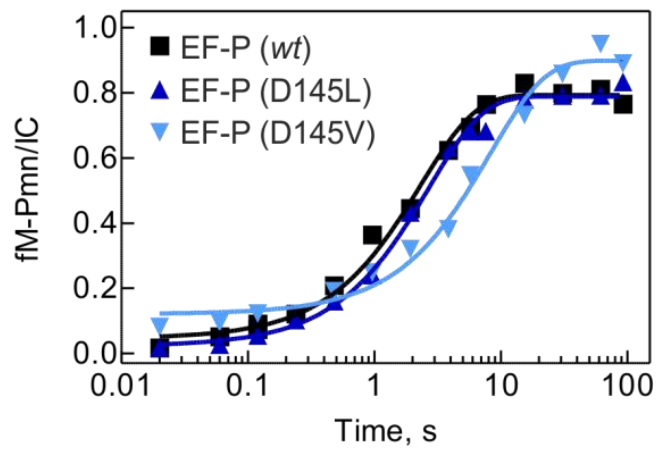

B

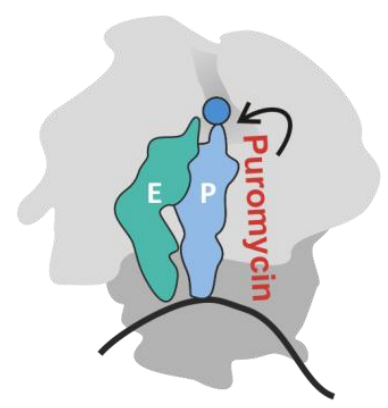

D

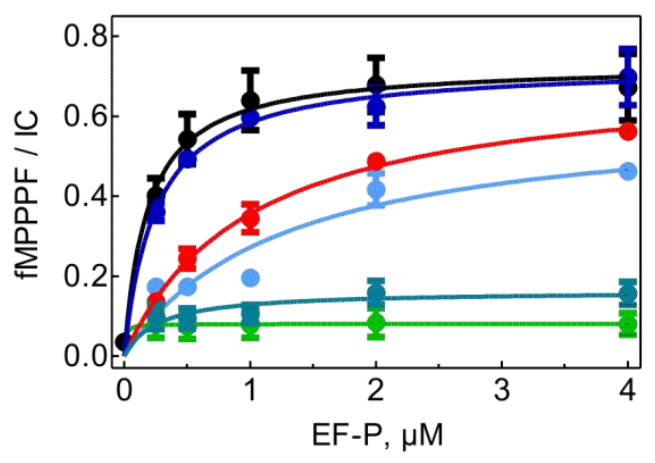

F

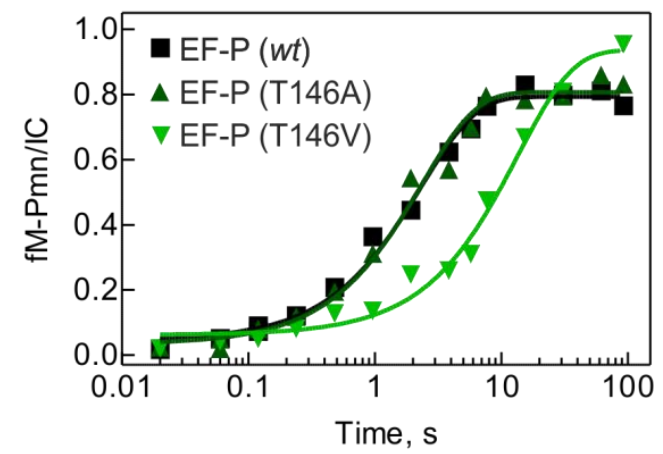

Figure 21: Influence of the E-site codon on EF-P facilitated catalysis.

A) Position of the EF-P domain III (d3) loop I, with residues G144, D145, and T146 of EF-P may interact with the E-site mRNA codon. Adopted from: Figure 8A.

B) Scheme of the puromycin assay using the aminonucleoside Pmn as A-site substrate analog.

C) Time courses of fMet-puromycin formation with ICs presenting an the lle codon AUA (IleIC, black circles)) or the Pro codon CCG (ProlC, red circles) in the E-site. The reaction was performed in the presence of sub-saturating concentrations of EF-P.

D) Activity of EF-P domain III loop I variants. Synthesis of the fMPPPF peptide in the presence of increasing concentrations of EF-P $w t$, black; loopI $\Delta 1$., blue; ${ }_{144} \mathrm{AAA}_{146}$, red; loopl $\Delta 2$, light blue; loopl $\Delta 4$, urquoise; and $\Delta$ domain III, green (adopted from (Huter et al., 2017)).

E) Time course of fMet-Pmn formation with EF-P (wt) (black squares) and EF-P mutants D145L (dark blue triangles) and D145V (light blue reverse triangles).

F) Time courses of fMet-Pmn formation with EF-P (wt) (black squares) and EF-P mutants T146A (dark green triangles) and T146V (light green reverse triangles).

With EF-P (wt), (D145L) and (T146A) similar reaction rates were obtained of about $0.43 \pm 0.04 \mathrm{~s}^{-1}$. The substitution of D145V and T146V showed a reduced $\mathrm{k}_{\mathrm{obs}}$ of about $0.12 \pm 0.02 \mathrm{~s}^{-1}$, which corresponds to the reported non-catalyzed reaction rate 
(Doerfel et al., 2013), suggesting that substitution of D145 or T146 with valine abolished EF-P activity, whereas EF-P (D145L) and (T146A) remained as active as EF-P (wt). The substitution of the charged aspartic acid with a hydrophobic residue did not reduce the activity of EF-P. Hence, it is unlikely that the side chains of $D$ and $T$ as such are important for the function. These conclusions are supported by the EF-P $\left({ }_{144} \mathrm{AAA}_{146}\right)$ variant which showed only a slight reduction in it activity. These findings underscore that the E-site codon does not affect EF-P activity, but rather the integrity or the conformation of loop I is important. This conclusion is in agreement with the findings concluded in section 2.2.1, in that the binding of EF-P is independent of an E-site codon interaction.

\subsubsection{The post-translational modification of EF-P}

The $(R)-\beta$-lysylhydroxylysine modification of EF-P lysine 34 is crucial for the functional activity of EF-P (Doerfel et al., 2013; Ude et al., 2013). While the hydroxylation of K34 showed only a minor effect on the ability of EF-P to facilitate peptide bond formation, the (R)- $\beta$-lysinylation had a major impact (1.3.2). Replacement of Lys34 by Ala abolishes the modification (chapter 1.3.7). To investigate whether the body of EF-P, independent of EF-P modification and lysine 34, can contribute catalysis, the biochemical activities of modified, unmodified EF-P and EF-P K34A were compared. In order to do so, the corresponding EF-P variants were used in a reconstituted-translation system using ICS programmed with an fMPPPF coding mRNA (Figure 22A).

A

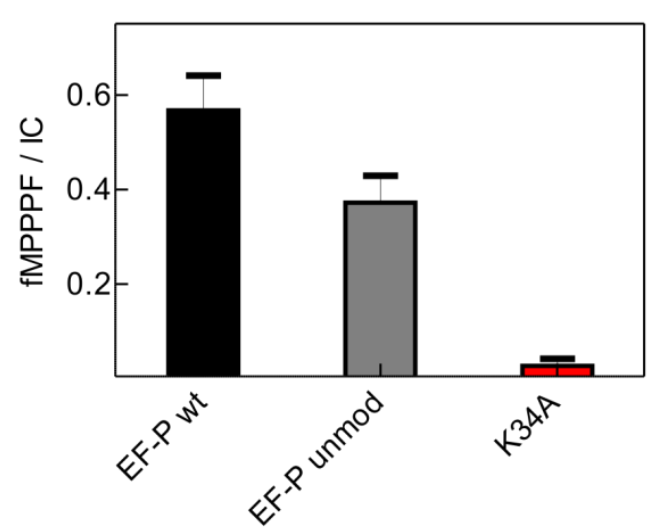

B

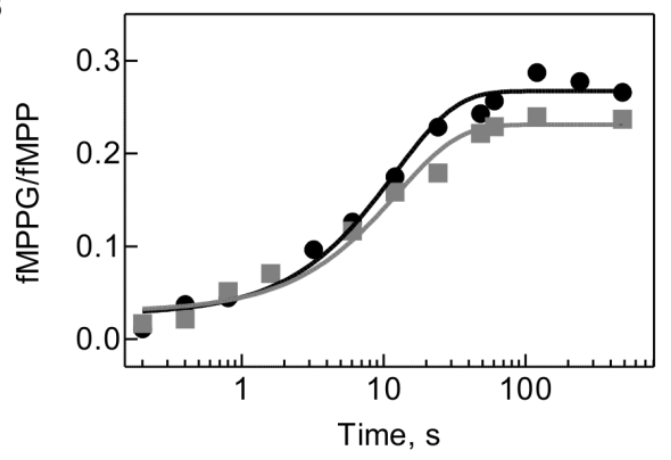

Figure 22: Effect of the modification of EF-P on peptide bond formation.

A) Activity of differently modified EF-P. Formation of FMPPPF in an in vitro translation system in the presence of saturating EF-P $(4 \mu \mathrm{M})$.

B) The time resolved reaction of POST(FMPP) with Gly-TC catalyzed by sub-saturating concentration of either fully modified EF-P (black) or unmodified EF-P (grey) was performed in a quench-flow apparatus. 
In the presence of fully modified EF-P $60 \%$ of the substrate reacted to the PMPPPF product Figure 22A). In agreement with previously reported data (Doerfel et al., 2013; Ude et al., 2013), the unmodified EF-P showed a reduced activity and yielded $40 \%$ of product. The EF-P variant $\mathrm{K} 34 \mathrm{~A}$ was initially reported to have a similar activity as unmodified EF-P (Ude et al., 2013). However, the translation assay showed no activity of EF-P K34A. Because the body of EF-P remained unchanged, the loss of activity results solely from the K34A exchange. However, the lack of modification did not change the rate of the puromycin reaction at sub-saturation EF-P concentrations (Figure 22B). Under sub-saturating conditions the observed reaction rate is sensitive to catalytic and binding contributions. The reaction rate was $0.08 \pm 0.01 \mathrm{~s}^{-1}$ in both cases. In the absence of EF-P a reaction rate of about $0.01 \mathrm{~s}^{-1}$ was reported, while under saturating concentrations of modified EF-P a reaction rate of about $1.3 \mathrm{~s}^{-1}$ was reported (Doerfel et al., 2013). Thus, the modification has only a minor contribution to binding, consistent with the conclusions presented in section 2.2.3.

We planned to use the catalytically inactive EF-P variant K34A in pulsed-chase experiments, to better understand the correlation between EF-P dissociation and assisted catalysis. This experiment addresses the question, whether the mechanism of EF-P facilitated peptide bond formation in one run- or requires several association events. However, the experiment failed. Because we showed that EF-P (K34A) is catalytic inactive, it is likely that the equilibrium dissociation constant of EF-P (K34A) was significantly underestimated. Notably, EF-P (K34A) was considered sofar as an equivalent of unmodified EF-P (Mohapatra et al., 2017; Ude et al., 2013). However, in this study and in collaboration with the Wilson group we could show that EF-P (K34A), in contrast to unmodified EF-P, is completely inactive (Huter et al., 2017). Hence, we investigated why EF-P (K34A) appears to be catalytic inactive and followed up on the potentially underestimated $\mathrm{K}_{\mathrm{d}}$.

In order to determine the binding affinity of EF-P K34A to the ribosome a EF-P(QSY) (K34A) labeling variant was established, and used in the EF-P binding assay (chapter 0). Surprisingly, with increasing concentration of labeled EF-P K34A no change in the fluorescent signal was monitored (Figure 23A). 
A

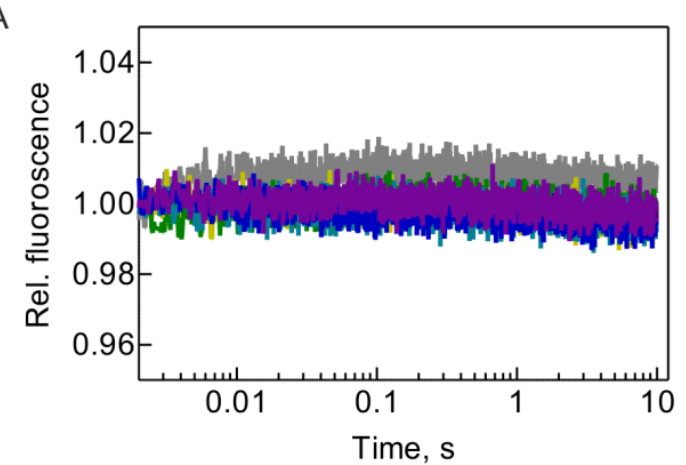

C

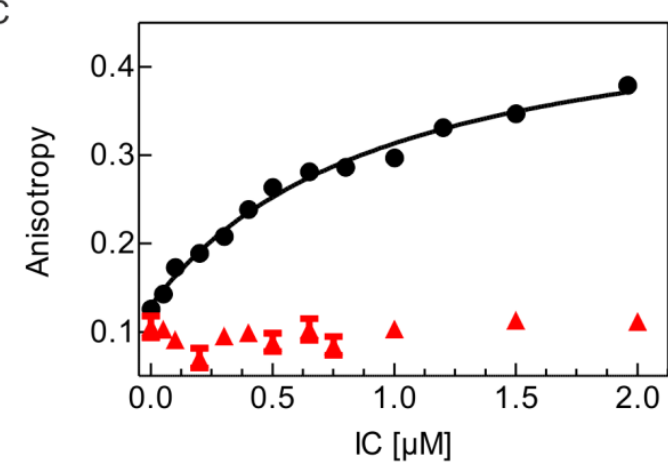

B

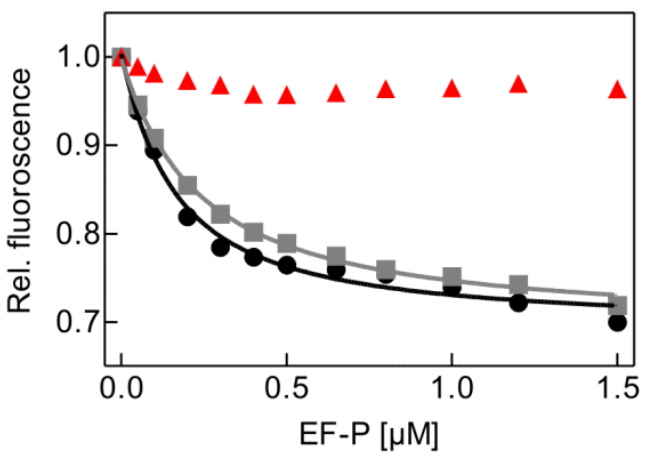

Figure 23: Characterisation of EF-P K34A.

A) EF-P binding assay using EF-P(QSY) (K34A). Experiments were carried out with IC(Alx) $(0.05 \mu \mathrm{M})$ and increasing concentrations of EF-P(QSY) (K34A): $0.25 \mu \mathrm{M}$ (ochre), $0.5 \mu \mathrm{M}$ (green), $1 \mu \mathrm{M}$ (turquoise), $1.5 \mu \mathrm{M}$ (blue) and $2 \mu \mathrm{M}$ (purple). Buffer was used for the donor-only trace (grey).

B) Titration of EF-P(QSY) (K34A) to IC(L33Alx) in a fluorimeter. The fluorescence change with increasing concentration of fully modified EF-P(QSY) (black), unmodified EF-P(QSY) (grey) and $E F-P(Q S Y)(K 34 A)$ (red) in equilibrium was plotted. The in equilibrium dissociation constant $K_{d}$ was calculated using a quadratic equation.

C) Equilibrium binding of EF-P(Alx) (K34A) to ICs monitored as a change of fluorescence anisotropy with increasing concentration of ICs, fitted with a quadratic equation. Black: EF-P(Alx) and red: EF-P(Alx)(K34A).

Because the obtained traces reflect the fluorescence donor trace, it is likely that EF-P K34A does not bind to the ribosome. In order to verify this finding, an equilibrium titration IC with EF-P(QSY) was performed. While modified and unmodified EF-P(QSY) showed a concentration-dependent reduction of the fluorescent signal, the addition of EF-P(QSY) (K34A) did not to change the fluorescent signal (Figure 23B). This supports the conclusion that EF-P(QSY) (K34A) might not bind to the ribosome. To exclude that the FRET-based binding assay fails to report on EF-P(QSY) (K34A) binding, an alternative assay was used to study binding. EF-P (K34A) was labeled with Alexa Fluor ${ }^{\text {TM }} 488$ and used for anisotropy measurements. Initiation complexes were titrated with EF-P(Alx) (K34A) and the anisotropy was monitored. While the anisotropy of EF-P(Alx) increased with an 
increasing concentration of IC, no change in anisotropy was observed using EF-P(Alx) (K34A) (Figure 23C). The measured anisotropy values for EF-P(Alx) (K34A) reflect the unbound state, with a high degree of rotational freedom of the dye. In conclusion, EF-P K34A does not facilitated peptide bond formation because it does not bind to the ribosome. In addition, the thermostability of EF-P (K34A) was compared with EF-P (wt) and EF-P (D66C) by ProteoPlex analysis. The preliminary analysis of EF-P variant K34A showed a reduced thermostability, whereas EF-P (wt) and the labeling variant D66C showed the same and higher thermostability (section: 5.4, Figure 30). These finding suggest, that EF-P (K34A) might be impaired in its fold. Taking these results together indicates that the body of EF-P is less important than expected and highlights the crucial role of Lys $\mathrm{K} 34$ for the activity of EF-P.

\subsubsection{The ribosomal protein $\mathrm{L} 1$}

Finally, we tested the role of L1 in the ability of EF-P to rescue translation on poly(Pro) sequences. ICs ( $w t$ and $\Delta \mathrm{L} 1$ ) programmed with an mRNA encoding fMPPPF were mixed with the components of the translation system and EF-P and the product yield was measured after $30 \mathrm{~s}$. With IC (wt) about $90 \%$ of the complexes produced the PMPPPF product, while with IC ( $\Delta \mathrm{L} 1) 60 \%$ of the complexes formed the product (Figure $24 \mathrm{~A}$ ). This result may indicate that ribosomes lacking L1 are somewhat less active in EF-P-dependent translation or the activity of EF-P is compromised. Next, the puromycin assay was used to determine the activity at sub-saturating conditions of EF-P allowing binding effects to contribute to the reaction rate. With IC (wt) $80 \%$ of the complexes formed the fM-Pmn product, while with IC ( $\Delta \mathrm{L} 1)$ only $20 \%$ of the complexes formed the product (Figure 24B). A smaller amplitude can result from side reactions, such as peptidyl-tRNA drop-off, or from a reduced activity of ribosomes lacking L1. The drop-off is unlikely to occur on ICs at the time scale of the experiment. The reaction rates were $0.43 \pm 0.04 \mathrm{~s}^{-1}$ for IC (wt) and $0.12 \pm 0.02 \mathrm{~s}^{-1}$ for IC $(\Delta \mathrm{L} 1)$, which is nearly 4 -fold slower in the absence of $L 1$. Because the deletion of L1 was reported to have no effect on the rate of in vitro translation(reviewed in: (Tobin, 2011)), hence these findings show that EF-P is less active in the absence of ribosomal protein L1. In order to verify these findings, the variants EF-P D145L and T146A, which in previous experiments showed the same activity as EF-P (wt), were tested in the puromycin assay using IC $(\Delta \mathrm{L} 1)$. Independent of the used variants very similar product 
amplitudes were obtained (Figure $24 C$ ), which match the product amplitude for IC $(\Delta \mathrm{L} 1)$ in Figure 24B. In line with that, also the reaction rates were the same $\left(k_{o b s}\right.$ of roughly $\left.0.01 \pm 0.02 \mathrm{~s}^{-1}\right)$.

To test the kinetic effect of L1 on EF-P activity upon synthesis of the natural EF-P-dependent motif fMPPG, we mixed Post(fMPP) (wt) or Post(fMPP) ( $\triangle \mathrm{L} 1)$ with sub-saturating concentrations EF-P and TC-Gly (Figure 24D). With Post(fMPP) (wt) the $\mathrm{k}_{\text {obs }}$ value of synthesis was $0.077 \pm 0.009 \mathrm{~s}^{-1}$ (Figure 23D). When L1 was lacking, the rate was reduced by 10 -fold to $0.008 \pm 0.001 \mathrm{~s}^{-1}$. The modification of EF-P did not have an effect at the given conditions. The reduction of the reaction rate in the absence of $L 1$ could potentially result from an altered binding of EF-P. This suggests that L1 is required for a preceding macroscopic positioning of EF-P, which in turn allows the modification to contribute to catalysis. However, we showed that the $K_{d}$ of EF-P binding to the ribosome was independent of L1. A more likely explanation is that L1 is involved in the correct positioning of EF-P, which in turn results in a productive conformation of the nascent-chain and the CCA-end of the tRNA. Overall, these findings indicate that $L 1$ is not important for EF-P binding, but rather affects the ability of EF-P to catalyze peptide bond formation. 
A

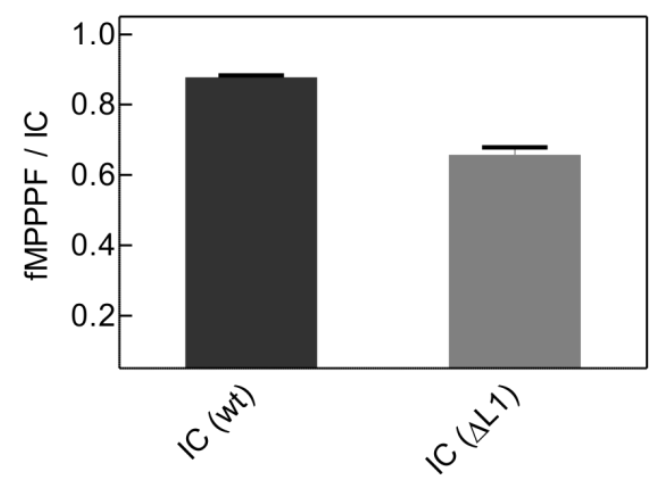

C

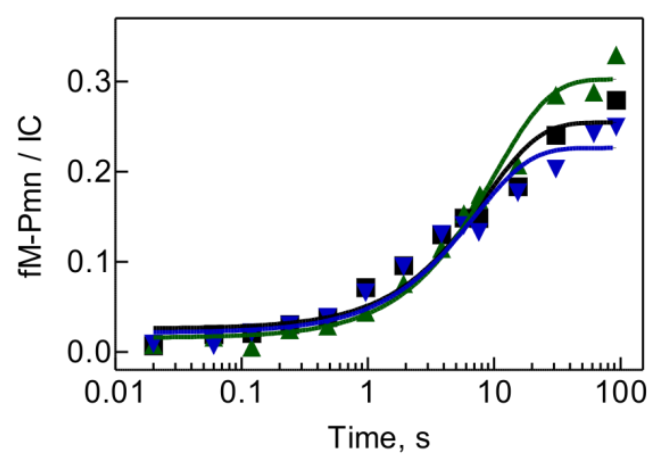

B

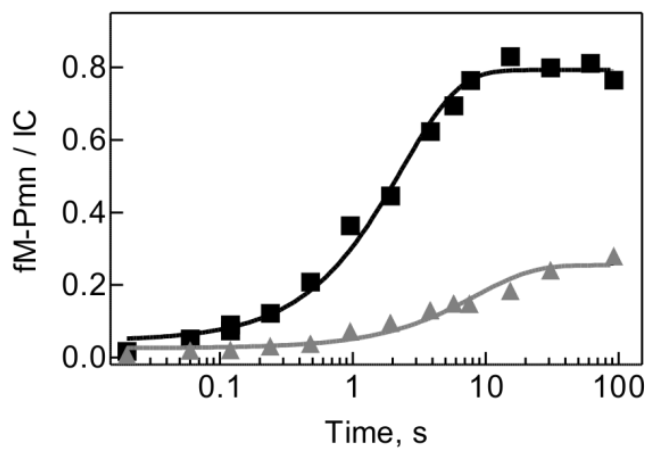

D

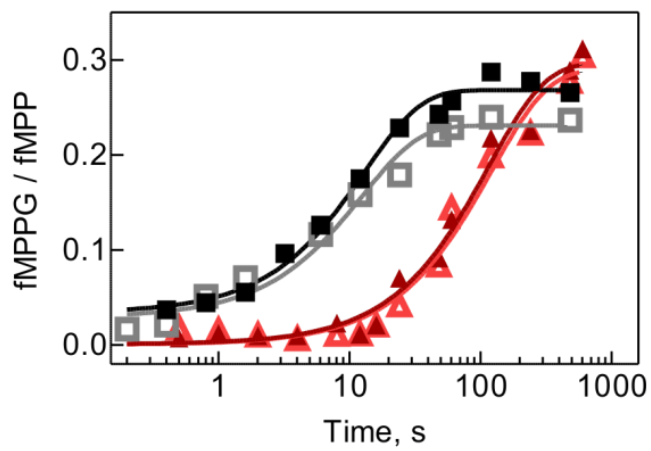

Figure 24: EF-P activity on ribosome lacking L1.

A) Translation efficiency. IC (wt or $\Delta \mathrm{L} 1$ ) programmed with mRNA encoding fMPPPF were mixed with the components of an in vitro translation system including EF-P $(2 \mu \mathrm{M})$.

B) Effect of L1 on EF-P-dependent peptide bond formation. Time courses of puromycin reaction was measured in the presence of sub-saturating concentrations of EF-P $(0.5 \mu \mathrm{M})$, supplemented with either IC ( $w t$, black) or IC ( $\Delta \mathrm{L} 1$, grey) using a quench-flow apparatus.

C) fMet-puromycin reaction on IC $(\Delta L 1)$ in the presence of EF-P domain III loop I variants. The experiments were performed as in B with EF-P (wt) black; EF-P (D145L), blue; and EF-P (T146A), green.

D) Catalysis of PPG synthesis with native and $\triangle \mathrm{L} 1$ ribosomes. The reaction of Post(fMPP) with Gly-TC, was performed in the presence of sub-saturating concentrations of EF-P $(0.5 \mu \mathrm{M})$ in a quench-flow apparatus. The following combination of post-complexes and EF-P were tested: Post(fMPP) ( $w t)$ in the presence of fully modified EF-P (black) and unmodified EF-P (grey), and Post(fMPP) ( $\triangle \mathrm{L} 1$ )in the presence of fully modified EF-P (dark red) and unmodified EF-P (light red).

\subsection{Biochemical characterization of the EFP-like protein}

The reported modifications of EF-P cover only $31 \%$ of all sequenced bacterial genomes. For the remaining 69\%, no homologs of known EF-P modifying genes were found. This leaves space for the existence of yet undescribed alternative modification and even EF-P paralog factors. In some cases bacteria have either an incomplete set of modifying enzymes, multiple isoforms of EF-P or no copy of the EF-P gene. This indicates that the necessity of the post-translational modification might be overcome by specialized EF-P orthologues (reviewed in: (Lassak et al., 2016; Rajkovic and Ibba, 2017)). One of these suggested EF-P paralogs is the E. coli EFP-like protein (YeiP) (Richards et al., 2012), which 
is also found in other gamma-proteobacteria. The EFP-like protein is 190 aa long, shares a large similarity with EF-P and together they form the "elongation factor P" family of proteins. Interestingly, no data on the structure or function of EFP-like protein is available at present. Literature only describes the turn-over of the EFP-like protein mRNA, which showed no feedback regulation by the protein level itself (Richards et al., 2012). For both, efp and yeiP, the same mRNA half-time of $1.6 \mathrm{~min}$ was reported. Despite the similar mRNA half-times they seem to be subject to different degradation mechanism, because only yeiP was shown to be targeted by the RNA pyrophosphohydrolase $\mathrm{RppH}$, whereas efp is not (Deana et al., 2008). It has been suggested that EF-P isoforms exists and the EFP-like protein might be one of them ((Richards et al., 2012) and reviewed in: (Lassak et al., 2016; Rajkovic and Ibba, 2017)). This is supported by the structural models which share a great similarity with EF-P (Figure 25A). This prompted us to test whether EFP-like protein can replace EF-P in protein synthesis. The key characteristics of EF-P are its ability to stabilize the P-site peptidyl-tRNA and to facilitate peptide bond formation between suboptimal substrates (Doerfel et al., 2013; Doerfel et al., 2015). For these studies, two tagged variants of the protein were prepared, the $\mathrm{N}$ - and $\mathrm{C}$ - terminally His-tagged EFP-like protein (EFP-like $\mathrm{N}$ and EFP-like $\mathrm{C}$ ).

The ability of the EFP-like protein to stabilize the P-site peptidyl tRNA was tested in an in vitro translation system, using ICs programmed with mRNA coding for FMPPG. The peptidy-tRNA drop-off was monitored by the amount of peptidyl-tRNA which remained bound to the ribosome in the presence or absence of EF-P and EFP-like protein (Figure 25B). In presence of EF-P, 90\% of peptidyl-tRNA was stably bound to the ribosome, whereas inthe absence of EF-P only about $40 \%$ of the peptidyl-tRNAs remained bound. The presence of N-terminally tagged EFP-like protein did not stabilize peptidyl-tRNA to a significant extent, while the C-terminally tagged EFP-like protein had a small stabilization effect. 
A

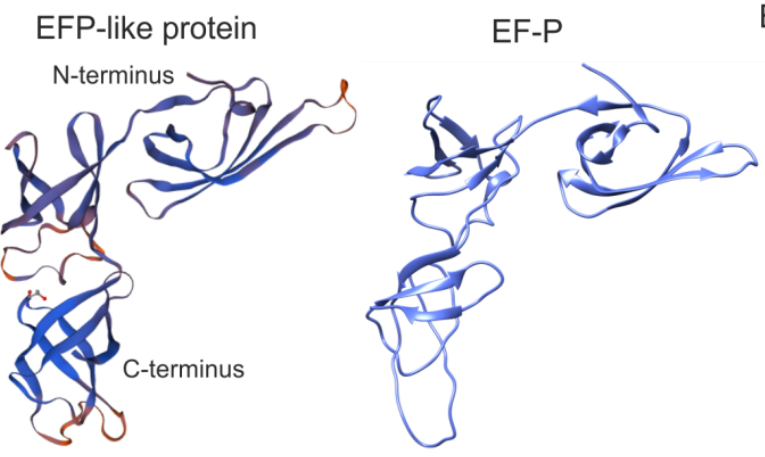

C

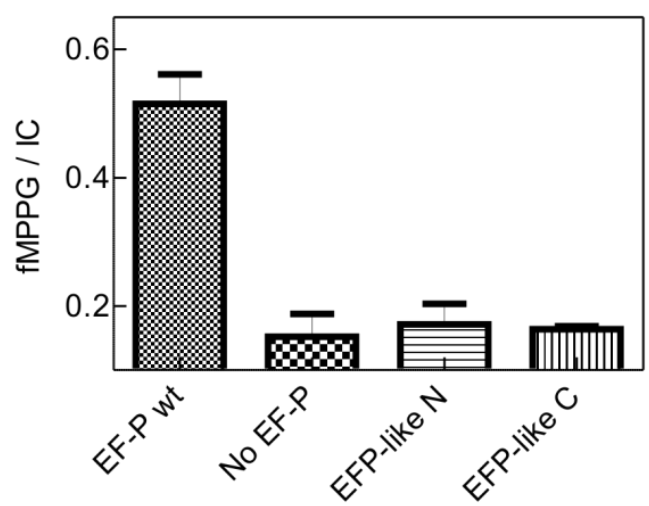

B

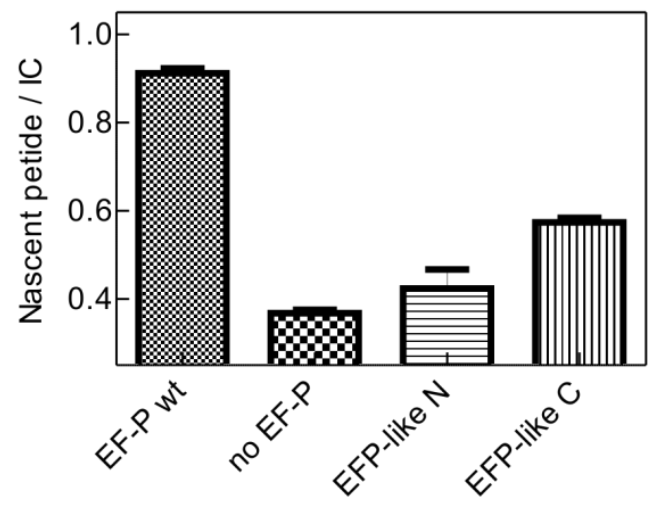

D

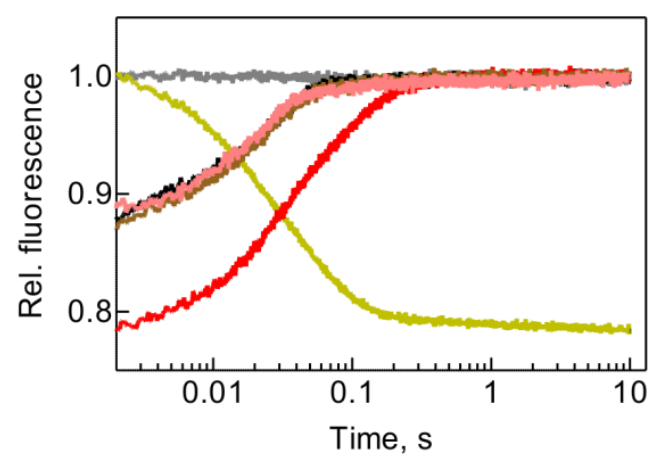

Figure 25: Binding and catalytic activity of the EFP-like protein in comparison to EF-P.

A) Structural comparison of EFP-like protein and EF-P. The structure of the E.coli EFP-like proteins was simulated by SwissModel, using the EF-P X-ray structure [PDB 1ueb.1A] as template. Cryo-EM structure of $E$. coli EF-P [PDB 6ENU].

B) Stabilization of the P-site peptidyl-tRNA by EF-P(wt) and EFP-like protein variants carrying either a N-or C-terminal His-tag. The ability to reduce peptidyl-tRNA drop-off was monitored by quantification of of ribosome-bound peptidyl-tRNAs by scintillation counting of $\left[{ }^{3} \mathrm{H}\right] \mathrm{fMet}$.

C) Comparison of the ability of EFP-like protein and EF-P to alleviate poly(Pro)-induced ribosome stalling. Initiation complexes programmed with an mRNA encoding fMPPG were used in an in vitro translation system lacking EF-P, or substituted with EF-P or either of the EFP-like protein variants. The product formation was monitored by $\left[{ }^{3} \mathrm{H}\right] \mathrm{Gly}$ incorporation.

D) Binding by the EFP-like variants and EF-P. The binding was monitored in chase experiments using EF-P and the EFP-like variants as chaser. EF-P(QSY) was pre-bound to IC(L33) and rapidly mixed with an excess of non-labeled EF-P (red), N-terminally (brown) and C-terminally tagged EFP-like protein (pink) or with buffer (black). The binding of EF-P(QSY) to IC(L33Alx) is shown in ochre and the donor only trace in grey.

To follow up on the potential ability to stabilize the peptidyl-tRNA, it was investigated whether the EFP-like protein is able to rescue translation of a poly(Pro)-stalled ribosomes, the key function of EF-P. For that, the product formation of $\mathrm{AMPPG}$ was monitored, using an in vitro translation system in the presence and absence of EF-P and EFP-like protein (Figure 25C). In the presence of EF-P 50\% of the complexes produced the fMPPG peptide. In the absence of EF-P no product ( $10 \%$ of the complexes formed the product). Supplementing the translation system with either of the EFP-like protein variants led, 
compared to the absence of EF-P, to no increase in product formation. The efficient formation of $\mathrm{FMPPG}$ is EF-P dependent (Doerfel et al., 2013) and the EFP-like protein did not compensate for the absence of EF-P it failed to rescue poly(Pro) induced stalling and hence, product formation.

Because, the EFP-like protein did not show any of the tested functions of EF-P, the question was raised, whether the EFP-like protein can bind to the ribosome. To address this question, both EFP-like protein variants were used in the EF-P binding assay as a chaser using a 20-fold excess (Figure 25D). The ability to hinder the re-association of EF-P(QSY) would indicate the ability of the EFP-like protein to bind to the ribosome. In the absence of any chaser an increase of the relative fluorescent from 0.9 to 1 was observed. Using unlabeled EF-P as chaser, resulted in an increase of the relative fluorescent from 0.8 to 1 . Using either of the EFP-like protein variants as chaser, resulted in an increase of the relative fluorescent from 0.9 to 1 . This resembles the trace without a chaser, suggesting that neither of the variants acted as a chaser. The regain in relative fluorescence is likely to result from EF-P(QSY) dissociation and reduced re-association, due to the dilution of the complexes in the experiment. These results indicates, that the EFP-like protein either has a very high $K_{D}$, hence the used excess was too little to act as a chaser or EFP-like protein does not bind to the ribosome. Our findings suggest that despite EFP-like proteins structural similarity to EF-P, it is no substitution for EF-P. Considering, that the model of EFP-like protein was created on the base of an EF-P X-ray structure, which is likely to bias the outcome of the simulation towards an "EF-P like" structure, questions the relation between EF-P and EFP-like protein even more. Nevertheless, none of the functional characteristics of EF-P were found for the EFP-like protein, neither the ability to binding to the ribosome, nor the stabilization of the P-site peptidyl-tRNA or the ability to alleviate poly(Pro) induced translational stalling. Hence, the role of the EFP-like protein remains still unknown.

\subsection{Mass-spectrometric quantification of EF-P in the cell}

To understand catalysis and binding of EF-P in the context of translation, it is important to know its cellular concentration. Different copy numbers were reported for EF-P. Early studies relying on 2D gel-electrophoresis and radioactive labeling suggested 5,000 copies 
of EF-P per E. coli cell (An et al., 1980). A more recent mass SF up to 40,000 copies of EF-P per cell depending on the growth conditions (Schmidt et al., 2016a). However, ribosomal proteins are challenging for such label-free quantifications, which have the assumption that after proteolysis the peptides of all proteins have a similar oberservability. Due to their specific environment within the ribosome they share little similarities with cytosolic proteins. In comparison to other proteins they are small and contain over-proportionally many lysine and arginine residues. The high abundance of these trypsin cleavage motifs results in a high abundance of very short tryptic peptides which are difficult to detect by mass spectrometry. As a result the reported concentrations for ribosomal proteins are not equimolar but scatter over orders of magnitude (Schmidt et al., 2016a). This prompted us to remeasure the concentration of EF-P relative to the ribosome. To account for such for such sequence specific differences we decided to establish correction factors and apply them to our label free mass spectrometric results. In two constructs EF-P was fused with an individual representative ribosomal proteins. Here the ribosomal proteins L4 and L17 were used (Figure 26A). After proteolysis, the fusion proteins guarantee an equimolar ratio of the peptides derived from EF-P and the used ribosomal protein in the mass-spectrometric analysis. Observable peptides for the correction factors were identified by untargeted data-dependent acquisition (DDA) and for more consistent quantifications in the lysate targeted by parallel reaction monitoring (PRM). The integrated areas of the peptide fragments were used to calculate individual correction factors between the observed ribosomal and EF-P peptides. These correction factors were applied to the corresponding peptide intensities determined in the E. coli cell-lysate (Figure 26B and C). 
A

\section{EF-P $\quad$ L17}

B

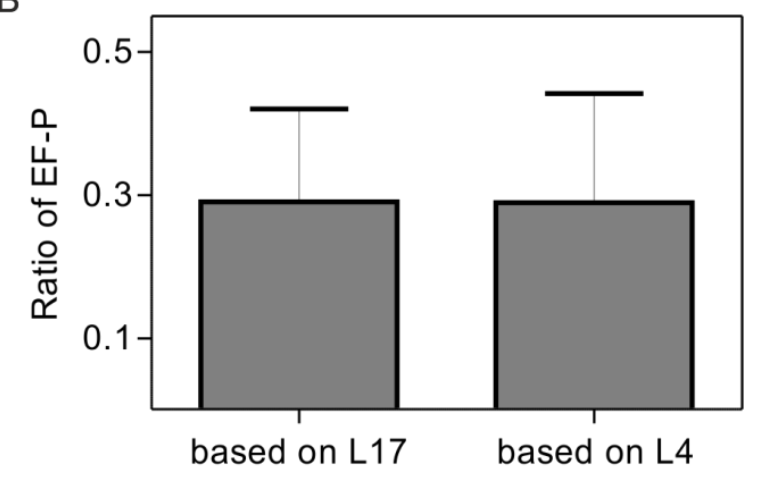

\section{$\begin{array}{ll}\text { EF-P } & \text { L4 }\end{array}$}

C

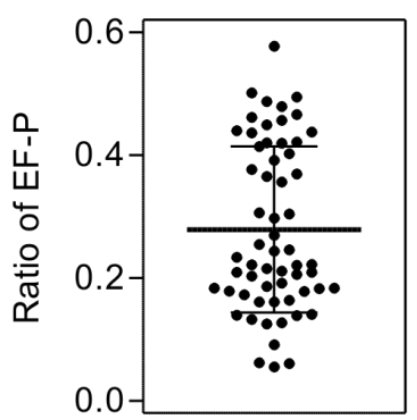

Figure 26: Mass-spectrometric quantification of EF-P.
A) Used fusion proteins.
B) Calculated ratio of EF-P to the ribosome based on ribosomal protein L17 and L4.
C) Individual stoichiometries between selected peptides EF-P and ribosomal peptides.

Average ratio of about 0.3 copies of EF-P per ribosome was determined. However, plotting the calculated ratio for the individual fragments revealed two sub-populations. One population shows a ratio of about 0.2 and the second population a ratio of about 0.4 . These differences are likely to arise from the fact that only two EF-P peptides could be reproducibly detected in the lysate which led to these slightly deviating quantification. Nevertheless, our finding of about of $0.3 \pm 0.1$ copies of EF-P per ribosome is consistent with previous reported EF-P copy number (Schmidt et al., 2016a). 


\section{Discussion}

The catalytic mechanism of EF-P is well understood (Doerfel et al., 2013; Doerfel et al., 2015; Glick et al., 1979; Glick and Ganoza, 1975; Huter et al., 2017; Katoh et al., 2016; Ude et al., 2013). When the ribosome encounters a run of Pro codons, it stalls after incorporation of the second proline and requires the help of EF-P to resume translation (Doerfel et al., 2013; Peil et al., 2013; Ude et al., 2013). These conclusions, which were initially obtained in vitro, are in the meantime supported by in vivo ribosome profiling data (Woolstenhulme et al., 2015). However, it remains unclear under which circumstances EF-P binds to the ribosome during translation, and whether EF-P is recruited to its target complexes. Here, we established an in vitro EF-P binding assay to study the dynamics of EF-P in its functional context, and to dissect which of its interactions are important for the recognition of substrate complexes.

The advantage of such an in vitro assay is the capability to break down the complexity of the reaction mechanism into individual kinetic steps, which can be studied separately. An example of such an approach is the dissection of translocation into multiple separately observed, loosely coupled events (Reviewed in: (Belardinelli et al., 2016b)), or of the mRNA decoding into a series of reversible and irreversible steps resulting in the incorporation of an amino acid into the nascent chain (Mittelstaet et al., 2011; Ranjan and Rodnina, 2017; Wohlgemuth et al., 2011; Wohlgemuth et al., 2010). Such work not only results in a conclusive overall mechanism, but also provides information about stable intermediate states which can be then isolated and their structure solved by e.g. cryo-electron microscopy or X-ray crystallography (Fischer et al., 2015; Ogle et al., 2001; Ogle et al., 2002; Schmeing et al., 2005; Schmeing et al., 2009; Voorhees et al., 2010; Weixlbaumer et al., 2008). The disadvantage of an in vitro system is the necessity to extrapolate the results to the in vivo context, where the observed step might undergo regulation or very different concentrations of the interaction partners are found ((Schavemaker et al., 2017) and reviewed in: (Rivas and Minton, 2016)). The proper extrapolation to the conditions in the cell requires that the in vitro translation system works at in vivo-relevant rates and with a fidelity similar to translation in vivo. Our translation system fulfills this requirement (Mercier and Rodnina, 2018; Rudorf et al., 2014). Furthermore, mathematical analysis of translation revealed that the rate constants derived at in vitro conditions can be extrapolated to in vivo conditions, and may be used 
to simulate complex experimental time courses of protein synthesis in vivo (Rudorf et al., 2014). Thus, it is likely that the elemental rates of individual EF-P interaction steps obtained from the in vitro system can be used to estimate the ribosome occupancy of different ribosome complexes during translation in cells. One of the main questions concerning EF-P binding is whether EF-P stochastically samples all ribosomes or whether certain structural recognition elements target EF-P to Pro-stalled ribosomes, and if so, how such a targeting could be regulated.

In contrast to other elongation factors such as EF-G and EF-Tu, EF-P is not required for each round of translation elongation. It has a highly specific functional context and assists the ribosome during translation of poly(Pro) motifs. Proline is a poor A- and P-site substrate (Doerfel et al., 2013; Muto and Ito, 2008; Pavlov et al., 2009; Wohlgemuth et al., 2008), causing the rate of peptide bond formation to be reduced to such an extent that the translation of consecutive proline codons results in the stalling of translation. By specifically stimulating the peptide bond formation between a di-prolyl-peptidyl-tRNA and a slow A-site substrate, EF-P alleviates the poly(Pro)-induced translational stalling and allows translation to proceed (Doerfel et al., 2013; Peil et al., 2013; Ude et al., 2013; Woolstenhulme et al., 2013). Because the function of EF-P is context dependent, it is likely that poly(Pro)-stalled ribosome complexes provide EF-P recognition motifs. These binding determinants would allow EF-P to discriminate between complexes requiring assistance and those which do not. X-ray crystallographic and recent cryo-electron microscopic studies have shown that EF-P binds the ribosome between the $E$ and $P$ site (Blaha et al., 2009; Huter et al., 2017), making a vacant $E$ site the prerequisite for EF-P binding that also kinetically controls the function of EF-P. In vivo studies showed that for other stalling events, EF-P had a negligible effect (Hersch et al., 2013; Woolstenhulme et al., 2013), either because no binding determinants were displayed or because EF-P does not alleviate the stalling at those sequences. To answer this question, a better understanding of EF-P binding is needed. This can be achieved by dissecting the contribution of each EF-P interaction element to binding and the activity of EF-P.

Structural studies showed that the conserved GDT-motif of the flexible loop I of domain III is within hydrogen bonding distance of the E-site codon (Huter et al., 2017). This indicates that the E-site codon may act as a recognition element providing a specific functional context during stalling on Pro-codon stretches. In fact, poly(Pro)-induced 
stalling occurs after incorporation of a second proline, hence poly(Pro)-stalled complexes always display a proline codon in the vacant $E$ site and a peptidyl-tRNA ${ }^{P r o}$ in the $P$ site. In contrast to EF-P, eIF5A lacks domain III and hence cannot interact with the E-site codon. Thus, the putative interactions between loop I of domain III of EF-P, even if they exist, are not evolutionary conserved. The recognition of specific codons has already been reported for release factors, which distinguish the stop codons using different peptide motifs (Ito et al., 2000). Proline is encoded by four codons of the CCN family and thus the flexible loop of domain III should recognize all four codons. We analyzed the affinity of EF-P variants, in which the residues contributing to the putative hydrogen bonds with the E-site codon were exchanged with hydrophobic residues. For the flexible loop EF-P variants which were tested, the affinity to the ribosome was not reduced (section 2.2.1). In a reverse experiment, the binding of EF-P $(w t)$ to different codons was evaluated (section 2.2.1). We found no indication that EF-P could distinguish between proline and non-proline codons or that altered E-site codon can hinder EF-P binding, as it was suggested by molecular dynamics simulations (Huter et al., 2017). Although the affinity of EF-P for initiation complexes was not affected, some EF-P variants show a highly reduced FRET efficiency in the binding assay (section 2.2.1). Because the FRET efficiency depends on the distance between the FRET donor and acceptor, as well as the relative orientation of their dipole moments (reviewed in: (Clegg, 2002)), this reduction indicates a different conformation or orientation of the bound EF-P or the dyes, respectively. Consistent with these observations, the interaction of EF-P and the $E$ site codon appears to that positions EF-P bound to the ribosome. For the activity of EF-P, however, the correct binding conformation might be essential, because EF-P stimulates peptide bond formation by positioning the peptidyl-tRNA in the peptidyl-transferase center in a catalytically active conformation (Doerfel et al., 2013; Doerfel et al., 2015; Huter et al., 2017).

This prompted us to test the reaction between initiation complexes and puromycin in the presence of the previously described EF-P variants (section 2.3.1). We found that variants D145V and T146V show a 4-fold reduced reaction rate $\left(0.12 \mathrm{~s}^{-1}\right)$ in comparison with EF-P $(w t)\left(0.43 \mathrm{~s}^{-1}\right)$. To extend on this effect, EF-P variants with alternative E-site codon interaction surfaces to alleviate poly(Pro)-induced stalling and found that they have reduced activities (Huter et al., 2017). With a stepwise removal of the interaction elements the product level is stepwise decreased. The deletion of G145 shows no 
reduction in its ability to alleviate poly(Pro)-induced stalling, likely because it has no side-chain which extends towards the mRNA and can contribute to a hydrogen bond. Notably, the disruption of all potential hydrogen bonds by exchanging all three conserved residues to alanine reduces the ability of EF-P to rescue translation by only $16 \%$, whereas the deletion of G145 and T146 causes a reduction by 30\%. The deletion of the entire GDT-motif and the upstream located amino acid resulted in the loss of activity, which was also observed for the deletion of the whole domain III. To analyze whether the E-site codon contributes to the activity of EF-P, we tested the reaction between initiation complexes displaying different E-site codons and puromycin (section 2.3.1). The absence of any rate differences $\left(0.43 \mathrm{~s}^{-1}\right.$ for lle and Pro E-site codon) indicates that neither the binding nor the function of EF-P were impaired. This data suggests that there is a robust and non-specific interaction of EF-P and the E-site codon, which does not strictly rely on the residues D145 and T146. Because the binding kinetics of EF-P are not affected by the interaction of EF-P with the E-site codon, the disturbance of the binding interface, however, resulted in a decreased activity of EF-P. Thus, the E-site codon might position EF-P and thereby indirectly steer the peptidyl-tRNA into a catalytically-active conformation.

The nature of the peptidyl-tRNA in the $\mathrm{P}$ site, or more specifically the D-arm of the tRNA, which is conserved in tRNA ${ }^{\text {Pro }}$ and $t R N A^{f M e t}$, is essential for the activity of EF-P and hence was suggested to be crucial for EF-P binding (Katoh et al., 2017a; Katoh et al., 2016). So far we have only studied the binding of EF-P to initiation complexes, containing $\mathrm{fMet}_{\text {-tRNA }}{ }^{\mathrm{fMet}}$, which provides a putative $\mathrm{D}$-arm interaction. This prompted us to compare the binding kinetics of EF-P to initiation, Post(fMF), Post(fMP) and Post(fMPP) complexes (section 2.2.1 and 2.2.2). We found that EF-P has similar rates of association of about 40 to $80 \mu \mathrm{M}^{-1} \mathrm{~s}^{-1}$ for the tested complexes. However, the dissociation rate is highly affected by the nature of the complex and varied between 90 and $1 \mathrm{~s}^{-1}$.

Interestingly, slower dissociation rates and therefore higher affinities of EF-P were found to correlate with its functional context. For Post(Phe) complexes we found an affinity of EF-P with a $K_{d}$ of about $1 \mu \mathrm{M}$, while EF-P was reported to have a negligible contribution to reaction between POST(Phe) and puromycin (Doerfel et al., 2013). However, for initiation and POST(Pro) complexes, to which reaction with puromycin EF-P had a significant contribution, the affinity of EF-P was slightly increased by four-fold, to a 
$\mathrm{K}_{\mathrm{d}}$ of about $0.3 \mu \mathrm{M}$. For the poly(Pro)-stalled ribosome POST(fMPP), whose reactivity was reported to be almost dependent on EF-P (Doerfel et al., 2013; Peil et al., 2013; Ude et al., 2013), the affinity was further increased 50 -fold to a $K_{d}$ of about $0.02 \mu \mathrm{M}$. These findings indicate that the binding of EF-P is, indeed, dependent on the nature of the peptidyl-tRNA. Despites its relevance for the activity of EF-P, the interaction of EF-P with the D-arm also appears to contribute to binding and helps to discriminate the functional context of EF-P. This would support a model in which EF-P samples all ribosome complexes with a vacant $E$ site. The presence of $t R N A^{P r o}$ or $t R N A^{f^{P e t}}$ in the P site allows for additional interactions, which are reflected in a 4-fold lowered $K_{d}$ value. Surprisingly, although the complexes POST(fMP) and POST(FMPP) share the same tRNA body and thus, the D-arm, a 10-fold higher affinity of EF-P for POST(fMPP) complexes was found. This indicates that neither the tRNA, nor its D-arm or the amino acid proline, are sufficient to explain the additional stabilization of EF-P. However, the presence of a second proline residue in the nascent-chain reduces its flexibility (reviewed in section 1.3.1). The increased rigidity reduces the conformational space sampled by the nascent-chain, therefore reducing the likeliness to react with an A-site substrate. In the case of a diprolyl-moiety, not only is the rigidity increased, but also the conformation of the nascent-chain is altered to avoid clashes of the -2 proline with the ribosome (Huter et al., 2017). In addition, molecular dynamics simulations predicted that the diprolyl-containing peptidyl-tRNA itself adopts an altered position and moves away from the A-site (Huter et al., 2017). Hence it appears likely that, while for the MMP-tRNA $^{\text {Pro a classic }}$ post-translocation state can be assumed, fMPP-tRNA ${ }^{\text {Pro }}$ adopts an atypical strained conformation. This alternative positioning of the peptidyl-tRNA seems to provide specific EF-P binding interactions, resulting in a lower dissociation rate. Overall, it seems likely that the increased affinity of EF-P for poly(Pro)-stalled ribosomes depends on multiple interactions and is not based on the recognition of individual binding determinants.

Interestingly, this mechanism seems not to be conserved for the yeast homolog of EF-P, elF5A. In comparison to elF5A, EF-P has a 20-fold lower affinity for initiation complexes (Table 5 \& (Rossi et al., 2016)). The concentration of elF5A in yeast is in a 1:1 ratio with the ribosome, as found for factors involved in each round of translation (Kulak et al., 2014; von der Haar, 2008). The cellular concentration of EF-P is still a matter of discussion, with a suggested ratio of 1:10 (An et al., 1980; Schmidt et al., 2016a), 
whereas our data indicates a 1:3 ratio. Both factors, elF5A and EF-P, were initially discovered due to their ability to facilitate peptide bond formation between fMet-tRNA $^{\text {fMet }}$ and puromycin (Benne and Hershey, 1978; Glick et al., 1979; Glick and Ganoza, 1975; Schreier et al., 1977), apart from their function to alleviate poly(Pro)-induced stalling (Doerfel et al., 2013; Gutierrez et al., 2013; Ude et al., 2013). In contrast to EF-P, elF5A was shown to have a more general role during translation (Gregio et al., 2009; Henderson and Hershey, 2011; Saini et al., 2009), due to its ability to alleviate non-poly(Pro)-stalled ribosome complexes and was also its involvement in peptide release (Pierson et al., 2016; Schuller et al., 2017). The broader substrate spectrum of elF5A might be explained by structural data. The body of EF-P was found to be in extensive contact with P-site tRNA (Blaha et al., 2009; Huter et al., 2017), allowing for the recognition of the D-arm of tRNA ${ }^{\text {Pro }}$ and tRNA ${ }^{\text {fMet }}$ by EF-P (Katoh et al., 2016). elF5A however, did not show such interaction with the peptidyl-tRNA (Melnikov et al., 2016; Schmidt et al., 2016b). These findings further support the idea that EF-P discriminates its functional context according to the D-arm of the peptidyl-tRNA.

The modification of EF-P plays an important functional role in vivo, as observed for the deletion strains of the modifying enzymes of EF-P (Abratt et al., 1998; Balibar et al., 2013; Bearson et al., 2011; Navarre et al., 2010; Peil et al., 2013; Zou et al., 2012). The modification was proposed to increase the affinity of EF-P (Doerfel et al., 2013) to the ribosome. In the yeast homolog, the modified elF5A was reported to have a 10 -fold higher affinity than unmodified elF5A (Rossi et al., 2016). Hence, we characterized the binding of unmodified EF-P to different post-translocation complexes (section 2.3.2). However, we found that the modification has no impact on the affinity of EF-P for different post-translocation complexes. The absence of the modification caused an approximately 2 -fold reduction in the binding and dissociation rate of EF-P. From a steric point of view, a lower rate of binding appears counterintuitive. Considering that the modification of EF-P extends an already protruded lysine residue and EF-P has to bind in a specific and confined space within the ribosome, the binding should instead be hindered by such a modification, whereas a more compact unmodified EF-P should be able to bind faster. On the other hand, the modification contributes a positive charge, which could facilitate binding to the ribosome. 
Aside from a putative contribution to binding, which we could not confirm, the modification of EF-P was identified as a main drive of EF-Ps entropic steering effect on peptide bond formation (Doerfel et al., 2013; Doerfel et al., 2015; Ude et al., 2013), suggesting that its main relevance lies in the catalytically active positioning of the peptidyl-tRNA. This is supported by molecular dynamics simulations which predicted that only in the presence of modified EF-P the carbonyl-carbon of the aminoacyl ester in the peptidyl-di-prolyl-tRNA ${ }^{\text {Pro }}$ and the $\alpha$-amino group of the A site get into close proximity, assembling a catalytically active pre-attack conformation (Huter et al., 2017). In addition, the stabilization of the peptidyl-tRNA was shown to be independent of the modification of EF-P, further supporting its particular function in positioning the tRNAs in a catalytically active orientation. However, EF-P is modified in two separate steps, first the $(R)-\beta$-lysinylation of $\mathrm{K} 34$ and second the hydroxylation of K34 by EpmC. While we could shed more light on the relevance of the lysinylation, the relevance of the hydroxylation remains still elusive.

Although the impact of EF-P hydroxylation on binding was not tested separately, our data suggest that none of the post-translational modifications has a significant impact on the binding kinetics of EF-P to the ribosome. Because the hydroxylation of EF-P neither contributes to the ability of EF-P to facilitate peptide bond formationy nor to binding, its relevance remains unknown. Its function might be deduced from its eukaryotic homolog, however. In yeast, the EF-P homolog eIF5A is first modified with deoxyhypusine, which in a proceeding step is transformed to hypusine (Park et al., 2010; Tong et al., 2009a; Wolff et al., 1990; Wolff et al., 2000). Interestingly, both modification levels showed the same ability to facilitate peptide bond formation, hence no further modification would be required (Park et al., 2011). However, because the first reaction is reversible, it was suggested that the second reaction removes the functional deoxyhypusine-modified elF5A from the substrate-product pool of DHS and thereby prevents the backward reaction to an unmodified elF5A (Park et al., 2003). A similar mechanism would be applicable for EF-P and its modifying enzymes, where the hydroxylation removes the $(R)$ - $\beta$-lysinylated EF-P from the substrate-product pool of EpmA, thus preventing the backward reaction to unmodified EF-P. However, this assumption still needs to be proven. Surprisingly, the EF-P variant K34A, which was initially described as a functional homolog of unmodified EF-P (Ude et al., 2013) shows no activity and no affinity to the ribosome 
(section 2.3.2). We obtained the same results for the EFP-like protein (section 2.4), which was suggested to be a functional paralog of EF-P ((Richards et al., 2012) and reviewed in: (Lassak et al., 2016; Rajkovic and Ibba, 2017)). Interestingly, the analogous mutation of $\mathrm{K} 34 \mathrm{~A}$ in yeast, $\mathrm{K} 56 \mathrm{~A}$, resulted in more than 20 -fold increase in the $\mathrm{K}_{\mathrm{d}}$, however, the authors did not comment on the stability of this elF5A variant (Rossi et al., 2016). For EF-P (K34A) the loss of its binding ability likely results from its thermodynamic instability and the resulting impaired folding (section: 2.2.3). The same assumption could be drawn for EF-P variants with mutations in residues Y183 and R186, which also showed a complete loss of function upon alanine exchange. The thermodynamic instability of EF-P is further supported by studies which showed that EF-P is the dominant aggregate in the cell under various stress conditions in the absence of relevant chaperones (Tomoyasu et al., 2001). These findings propose that EF-P had to evolve such a thermodynamically-unstable fold to be able to achieve such high specificity.

The question remains as to whether EF-P is actively recruited or whether its dissociation is guided by the ribosome. For deacalyted tRNAs the clearance from the E site is assisted by an interaction with the ribosomal protein L1 (Bock et al., 2013; Trabuco et al., 2010). The movement of L1 coincides with the rotation of the ribosome and with the different tRNA hybrid states (Cornish et al., 2009). Analogous to the L1-tRNA interaction, L1 was found to interact with the ribosome-associated EF-P (Blaha et al., 2009; Huter et al., 2017). Thus, L1 might be involved in the recruitment and/or dissociation of EF-P. Also for elF5A the interaction with L1 was proposed to stabilize its binding (Barbosa et al., 2016; Melnikov et al., 2016; Schmidt et al., 2016b). However, the binding affinity of EF-P to initiation complexes containing or lacking $L 1$ is similar (section 2.2.4). Either the observed interactions between EF-P and L1 influences the binding and dissociation rates to the same extent or the favorable energy of L1-EF-P binding is compensated by losses in e.g. conformational freedom or solvation energy. However, L1 is important for EF-P-dependent catalysis of peptide bond formation, because in the absence of L1, the observed reaction rate is reduced 4 -fold (section: 2.3 .3 ). Thus, L1 is likely to be involved in the positioning of EF-P or the peptidyl-tRNA on the ribosome, and important for a catalytically active conformation.

Compared to other translation factors, EF-P has neither a particularly high affinity for the ribosome, nor particularly fast or slow binding rates. For non-poly(Pro)-stalled 
ribosome complexes, the binding and dissociation rates of EF-P are more similar to that of EF-Tu and EF-G than to initiation or release factors (Table 5), which have 10- to 100-fold higher affinities than EF-P (Hetrick et al., 2009). EF-G and EF-Tu are recruited to the A site of the ribosome by the ribosomal protein L7/L12 (Bock et al., 2013; Diaconu et al., 2005; Kothe et al., 2004) to form an initial binding complex. Comparing EF-P with other elongation factors shows that the association rates and the affinity of EF-Tu and EF-G in the initial complexes are similar to that observed for EF-P binding to e.g. the initiation complex (Rodnina et al., 1997; Rudorf et al., 2014). The similar rates of initial complex formation by different elongation factors may have evolved to support a high rate of translation elongation, in particular allowing for rapid scanning of different ribosome complexes by elongation factors.

After initial binding, EF-Tu (as a part of the ternary complex with aa-tRNA and GTP) and EF-G are stabilized on the ribosome through specific recognition or engagement steps (reviewed in: (Belardinelli et al., 2016b; Wohlgemuth et al., 2011)). Such engagement steps proceed through the establishment of additional (or new) interactions. As a result, the apparent affinity of EF-Tu and EF-G is increased and the dissociation of EF-G and EF-Tu results from GTP hydrolysis and $\mathrm{P}_{\mathrm{i}}$ release. Analogously, EF-P bound to the ribosome forms additional interactions with tRNA ${ }^{\text {Pro }}$ in the P site (section: 2.2.2 and (Huter et al., 2017; Katoh et al., 2016)). In contrast to EF-G and EF-Tu, it has no regulating GTPase activity inducing dissociation. Notably, the spontaneous dissociation rate of EF-P from the functional complexes with fMPP-tRNA appears rather low, $1 \mathrm{~s}^{-1}$. One can envisage that during the ongoing translation, EF-P is actively removed from the $E$ site during translocation by the P-site tRNA which moves through the $E$ site on its way out of the ribosome. The release of EF-P could also be indirectly driven by the formed peptide bond, which relaxes the functional context resulting in a higher dissociation rate as found for the non-functional context. 
Table 5: Binding kinetics of ribosome binding factors

\begin{tabular}{|c|c|c|c|c|}
\hline & $\mathrm{k}_{\text {on }}\left[\mu \mathrm{M}^{-1} \mathrm{~s}^{-1}\right]$ & $\mathrm{k}_{\text {off }}\left[\mathrm{s}^{-1}\right]$ & $\mathrm{K}_{\mathrm{d}}[\mu \mathrm{M}]$ & Cellular conc. $[\mu \mathrm{M}]$ \\
\hline EF-P (modified) & 71 & 25 & 0.3 & $5-20^{i \& j}$ \\
\hline (unmodified) & 42 & 9 & 0.2 & ND \\
\hline EFP-like protein & ND & ND & ND & $1.3^{k}$ \\
\hline EF-G & $140^{\mathrm{a}}$ & 70 & 0.2 & 10 \\
\hline EF-Tu (initial binding) & $175^{b}$ & 700 & 4 & $90-100^{h}$ \\
\hline IF1 (30S IF3/IF2) & $12^{c}$ & 0.02 & 0.002 & $2.5^{\mathrm{g}}$ \\
\hline IF2 & \multirow[b]{2}{*}{$200-1000^{c}$} & \multirow[b]{2}{*}{30} & \multirow[b]{2}{*}{$0.03-0.2$} & $3^{g}$ \\
\hline IF3 & & & & $2^{g}$ \\
\hline RF1 (UAA binding) & $34^{d}$ & $<0.1$ & $<0.0035$ & NA \\
\hline RF2 & NA & NA & NA & NA \\
\hline RF3 & NA & NA & NA & NA \\
\hline elF5a (modified) & NA & NA & $0.01^{e}$ & $8-15^{f}$ \\
\hline (unmodified) & NA & NA & $0.2^{\mathrm{e}}$ & NA \\
\hline
\end{tabular}

The binding of EF-P, as for most other translation factors, appears to be kinetically controlled. The kinetic window for EF-P binding is defined by the occurrence of a vacant E site, which normally does not occur during translation with an average speed of 10-20 amino acids per second. At such speed of translation the E site will always be fully or partially occupied by a dissociating tRNA, which would hinder the binding of EF-P. Surprisingly, an in vivo study reported that $30 \%$ of EF-P co-localizes with the riposome, which exceeds the expected frequency of poly(Pro)-stalling motifs in the E. coli, indicating that EF-P binds the ribosome also in the absence of proline-stalling motifs (Mohapatra et al., 2017). However, in a recent study it was shown that the average speed of translation is a statistical value that does not necessarily reflect the individual translation rates at codon resolution (Haase et al., 2018). Thus, individual slow elongation steps might lead to the occasional occurrence of a vacant $E$ site. In addition to the potentially vacant $E$ sites, 
our data shows that the binding of EF-P is not restricted to its functional context. Hence, EF-P can also bind to other paused or stalled ribosomes without contributing to catalysis.

EF-P-dependent stalled complexes remain associated with EF-P longer than those with a non-functional context. The comparison of the rates of EF-P accelerated peptide bond formation with the binding kinetics of EF-P reveals that at physiologic EF-P concentration its association is fast, while EF-P's dissociation rates match the rate of peptidyl transfer. The reaction of POST(fMP) with TC-Gly, which is 10-fold accelerated by EF-P, proceeds in the presence of EF-P at $33 \mathrm{~s}^{-1}$ (Doerfel, 2016). EF-P binds to POST(fMP) at a rate of $71 \mu^{-1} \mathrm{~s}^{-1}$ and dissociates at $25 \mathrm{~s}^{-1}$ (section 2.2.2). The primary functional context of EF-P, POST(FMPP), reacts with TC-Gly at a rate of $1 \mathrm{~s}^{-1}$, whereas EF-P binding occurs at a rate of $50 \mu \mathrm{M}^{-1} \mathrm{~s}^{-1}$ and the dissociation occurs at $1 \mathrm{~s}^{-1}$ ((Doerfel, 2016) , section 2.2.2). The correlation between the dissociation rates of EF-P and rate of EF-P-assisted peptide bond formation indicates that the dissociation of EF-P might be accelerated after peptidyl transfer by loss of its binding determinants. This finding also excludes the model of EF-P assisted catalysis in which EF-P has to bind multiple times to the stalled-complexes before the stalling is alleviated. Our data suggests that a single binding event is sufficient to position the peptidyl-tRNA in a catalytic active conformation, and that EF-P stays bound until peptide bond formation occurs. However, this conclusion is derived from in vitro data using complexes with a very short nascent chain and we cannot exclude that they adopt a state which does not fully reflect the in vivo situation. In the cell, the binding of EF-P might be influenced by other factors and we would find longer nascent chains. It was shown that the length and amino acid sequence of the nascent chain can have a significant effect on the translation rate and positioning of the nascent-chain itself (Reviewed in : (Rodnina, 2016)), (Huter et al., 2017; Katunin et al., 2002; Rudorf et al., 2014)).

In summary, we found that EF-P binds to all ribosome complexes that provide an empty $E$ site, almost independently of the individual predicted interactions with RRNA $^{\text {Pro }}$ or the E site codon. In non-stalling cases EF-P will rapidly dissociate from the ribosome with a rate that is compatible with translation elongation. However, in a poly(Pro) context the binding of EF-P is further stabilized. The resulting slow dissociation of EF-P matches the rate peptide bond formation of poly(Pro)-stalled ribosomes with slow A-site substrates. As a result the residence time of EF-P in poly(Pro)-stalled complexes is 
increased and allows ribosome complexes to overcome stalling and proceed with elongation in a single run. After peptide bond formation, the strained complex might relax, thus lowering the affinity for EF-P. In this scenario EF-P would be recruited to stalled complexes and released after peptide bond formation, minimizing the impact of stalling and EF-P action on translation speed and cellular fitness. 


\section{Materials and Methods}

\subsection{Equipment and consumables}

Table 6: List of equipment

\begin{tabular}{|c|c|}
\hline Device & Supplier \\
\hline Acrodisc 25 mm Syringe Filter & Pall Life Science \\
\hline ÄKTA FPLC & GE Healthcare \\
\hline ÄKTA Explorer & GE Healthcare \\
\hline Allegra $X-22 R$ centrifuge & Beckmann Coulter \\
\hline Avanti J-26 XP centrifuge & Beckmann Coulter \\
\hline Avanti J-30l centrifuge & Beckmann Coulter \\
\hline Benchtop centrifuge $5415 \mathrm{R}$ and $5810 \mathrm{R}$ & Eppendorf \\
\hline Bio-vision imaging system & Peqlab Biotechnologie \\
\hline Cell densitity meter Ultrospec 10 & GE Healthcare \\
\hline Cellulose nitrate filter, $0.45 \mu \mathrm{m}, 25 \mathrm{~mm}$ & Sartorius \\
\hline Amicon $^{\circledR}$ Ultra 5 kDA cut-off & Merck Millipore \\
\hline Digital sonifier cell disruptor W-250D & Branson \\
\hline Dounce tissue grinder & Wheaton \\
\hline Easy nLCII chromatography system & Thermo Fisher Scientific \\
\hline Emulsiflex C-3 homogenizer & Avestin \\
\hline Micro Time 200 & PicoQuant \\
\hline FLA900 fluorescence imager & Fuji Film \\
\hline ProteoPlex & ProteoPlex GmbH \\
\hline HPLC Fluorescence detector 2475 & Waters \\
\hline HPLC unit 2695 & Waters \\
\hline HPLC UV/VIS detector 2489 & Waters \\
\hline Innova 44 incubator shaker & New Brunswick \\
\hline Intelli-mixer & ELMI Ltd. \\
\hline JA-30.1 rotor & Beckmann Coulter \\
\hline JLA-8.1000 rotor & Beckmann Coulter \\
\hline Lambda Bio+ UV/VIS spectrometer & Perkin Elmer \\
\hline Liquid scintillation counter TriCarb 3110 TR & PerkinElmer \\
\hline Low retention $0.5 \mathrm{ml}$ tubes & Eppendorf \\
\hline D-Tube ${ }^{\mathrm{TM}}$ Dializer Midi, MWCO 3.5 kDa & Merck Millipore \\
\hline
\end{tabular}


Milli-Q Advantage A10

MLA-130 rotor

Nanodrop 2000

Optima XPN centrifuge

PCR Thermocylcer

inoLAB Sentix81

inoLAB pH720

Plates incubator INE600

Q-Exactive hybrid mass spectrometer

Q-Exactive Plus hybrid mass spectrometer

Savant SPD111V SpeedVac

SDS-PAGE electrophoresis chamber

SpeedVac vacuum concentrator SPD121P

Stopped-flow cut-off filter KV500

Stopped-flow SX20D spectrometer

SX4250 rotor

Syringe filter: $0.2 \mu \mathrm{m} \& 0.45 \mu \mathrm{m}$

Thermomixer comfort

Ti-50.2 rotor

TLA-100 rotor

TLS-55 rotor

TSQ Quantiva triple quadrupole mass spectrometer

TSQ Vantage triple quadrupole mass spectrometer

Ultimate 3000RSLC

Ultracentrifuge Optima L-100 XP

Vivaspin ${ }^{\circledR} 205 \mathrm{kDa}$

Vortex Genie 2

Water bath RE104 and E100
Merck Millipore

Beckmann Coulter

Peqlab Biotechnologie

Beckmann Coulter

Peqlab Biotechnologie

WTW

WTW

Memmert

Thermo Fisher Scientific

Thermo Fisher Scientific

Thermo Fischer Scientific

Bio-Rad

Thermo Fischer Scientific

Schott AG

Applied Photophysics

Beckmann Coulter

Sartorius

Eppendorf

Beckmann Coulter

Beckmann Coulter

Beckmann Coulter

Thermo Fisher Scientific

Thermo Fisher Scientific

Thermo Fisher Scientific

Beckmann Coulter

Sartorius

Scientific Industries

Lauda

\subsection{Chemicals}

All chemicals were purchased from Merck, Merck Millipore or Sigma Aldrich, if not indicated elsewhere. HPLC grade components were used for chromatographic separation. The dyes, enzymes and consumables used for this study are listed in Table 7. 
Table 7: Dyes, enzymes and consumables

\begin{tabular}{|c|c|}
\hline Product & Supplier \\
\hline AlexaFluor 488-mal & Thermo Fisher Scientific \\
\hline AlexaFluor 655-mal & Thermo Fisher Scientific \\
\hline Atto540Q-mal & Atto-Tec \\
\hline BodipyFL- $C_{1}-I A$ & Life technologies \\
\hline BSA & Life technologies \\
\hline Chymotrypsin & Thermo Fisher Scientific \\
\hline CMP & Jena Bioscience \\
\hline cOmplete Protease Inhibitor EDTA free & Roche \\
\hline DNase & Jena Bioscience \\
\hline dNTPs & New England Biolabs \\
\hline Dpnl & New England Biolabs \\
\hline Endoprotease - GluC & Roche \\
\hline Endoprotease - LysC & Roche \\
\hline GTP & Jena Bioscience \\
\hline Irga-Safe & Perkin Elmer \\
\hline NucleoSpin PCR cleanup kit & Machery-Nagel \\
\hline NucleoSpin Plasmid kit & Machery-Nagel \\
\hline Phusion Polymerase & Thermo Fisher Scientific \\
\hline Proline & Sigma Aldrich \\
\hline$\left[{ }^{14} \mathrm{C}\right]$ Proline & Perkin Elmar \\
\hline Puromycin & Sigma Aldrich \\
\hline Pyrophosphatase (PPase) & Lucigen \\
\hline Pyruvate kinase & Roche \\
\hline QSY-35-iodacetamide & Life technologies \\
\hline QSY-7-maleimde & Life technologies \\
\hline QSY-9-malimde & Life technologies \\
\hline QuickZint 361 & Zinnser Analytic \\
\hline RNAsin & Promega \\
\hline T5 Exonuclease & New England Biolabs \\
\hline T7 RNA polymerase & New England Biolabs \\
\hline Taq DNA ligase & Biozymes \\
\hline
\end{tabular}




\subsection{Chromatographic columns}

The chromatographic columns used in this study are listed in Table 8.

Table 8: List of columns

\begin{tabular}{ll}
\hline Columns & Supplier \\
\hline Chromolith ${ }^{\circledR}$ Performance RP-8e & Merck \\
HiTRAP Q $5 \mathrm{~mL}$ & GE Healthcare \\
HiTRAP HP SP $1 \mathrm{~mL}$ & GE Healthcare \\
LiChroCart 250-10, LiChrospher WP300 RP-18 & Merck \\
Protino Ni-IDA Resin & Macherey-Nagel \\
Protino column $35 \mathrm{ml}$ & Macherey-Nagel \\
Reprosil-Pur $120 \mathrm{C} 183 \mu \mathrm{m}$ column & Dr Maisch \\
Resource S & GE Healthcare \\
Superdex 75 10/300 GL & GE Healthcare \\
Superdex 75 Hiload 26/60 & GE Healthcare \\
\hline
\end{tabular}

\subsection{Software and applications}

The software used for data acquisition, analysis and depiction are listed in Table 9.

Table 9: List of software

\begin{tabular}{ll}
\hline Software & Provider \\
\hline Adobe Illustrator CS5 & Adobe Systems \\
CorelDRAW X7 & Corel \\
DNA Star Lasergene & DNASTAR Inc. \\
GraphPad Prism 5.0 & GraphPad Software \\
KinTecExplorer Pro 6.2.1 & KinTek Corporation \\
MATLAB & The MathWorks \\
MultiGauge 2.0 & Fujifilm \\
MaxQuant software 1.5.2.8 & Max Planck Institute of Biochemistry \\
Pymol 1.5 & Schrödinger
\end{tabular}


Skyline 3.5

TableCurve 2D v5.01

UCSF Chimera 1.10.1

Xcalibur 2.2
MacCoss Lab Software

Systat Software Inc.

Schrödinger

Thermo Fischer Scientific

\subsection{Buffers}

All buffers used in this study are listed in Table 10.

Table 10: Buffers

\begin{tabular}{|c|c|}
\hline Buffer & Chemical composition \\
\hline Reaction buffer $\left(\right.$ TAKM $\left._{7}\right)$ & $\begin{array}{l}50 \mathrm{mM} \mathrm{Tris}^{-} \mathrm{HCl}, \mathrm{pH} 7.5 \text { at RT } \\
70 \mathrm{mM} \mathrm{NH}_{4} \mathrm{Cl} \\
30 \mathrm{mM} \mathrm{KCl} \\
7 \mathrm{mM} \mathrm{MgCl}_{2}\end{array}$ \\
\hline High-Fidelity buffer (HiFi) & 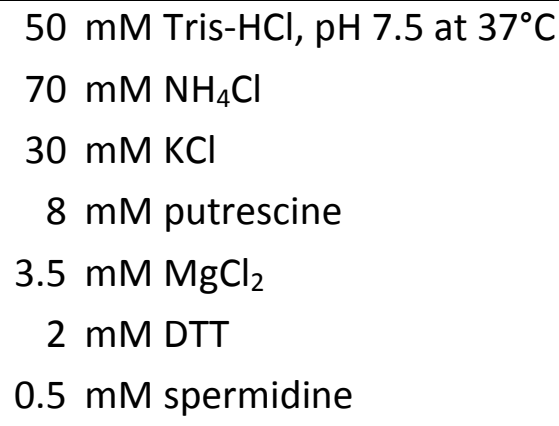 \\
\hline $\begin{array}{l}\text { HiFi compensation buffer (TAK } \\
\text { SPD) }\end{array}$ & $\begin{array}{l}50 \mathrm{mM} \mathrm{Tris}-\mathrm{HCl}, \mathrm{pH} 7.5 \text { at RT } \\
70 \mathrm{mM} \mathrm{NH}_{4} \mathrm{Cl} \\
30 \mathrm{mM} \mathrm{KCl} \\
16 \mathrm{mM} \text { putrescine } \\
4 \mathrm{mM} \mathrm{DTT} \\
1 \mathrm{mM} \text { spermidine }\end{array}$ \\
\hline In-vitro transcription buffer (TRAB) & $\begin{array}{l}40 \mathrm{mM} \mathrm{TRIS}, \mathrm{pH} 7.5 \text { at } 37^{\circ} \mathrm{C} \\
15 \mathrm{mM} \mathrm{MgCl}_{2} \\
10 \mathrm{mM} \mathrm{NaCl} \\
2 \mathrm{mM} \text { spermidine }\end{array}$ \\
\hline
\end{tabular}




\begin{tabular}{lc}
\hline Aminoacylation buffer & $50 \mathrm{mM} \mathrm{HEPES}, \mathrm{pH} 7.5$ at RT \\
& $75 \mathrm{mM} \mathrm{KCl}$ \\
& $35 \mathrm{mM} \mathrm{MgCl}$ \\
& \\
\hline TK-labeling buffer & $20 \mathrm{mM} \mathrm{TRIS}, \mathrm{pH} 8.3 / 7.3$ at RT \\
& $100 \mathrm{mM} \mathrm{KCl}$ \\
& $89 \mathrm{mM}$ TRIS base pH 8.0 at RT \\
& $89 \mathrm{mM}$ Boratic acid \\
& $2 \mathrm{mM}$ EDTA \\
& \\
\hline TBE & $40 \mathrm{mM}$ TRIS-acetate pH 8.0 at RT \\
& $1 \mathrm{mM}$ EDTA \\
& \\
\hline TAE & $25 \mathrm{mM}$ TRIS base \\
& $200 \mathrm{mM}$ glycine \\
& $0.1 \%$ SDS \\
\hline SDS-PAGE running buffer & $10 \%$ ethanol \\
& \\
& \\
&
\end{tabular}

\subsection{Bacterial and eukaryotic strains}

All strains used in this study are classified in biosafety level BSL-1 and were handled accordingly. For genetic subcloning and molecular biology work the $E$. coli strains $\mathrm{DH} 5 \alpha$ and NovaBlue were used. Protein overexpression was performed in E. coli BL21 (DE3) cells. Mass spectrometric proteome studies were based on E. coli MG1635, as well as $S$. cerevisiae S288C cells. Bacteria were cultivated in LB broth $(10 \mathrm{~g} / \mathrm{L} \mathrm{NaCl}, 10 \mathrm{~g} / \mathrm{L}$ tryptone and $5 \mathrm{~g} / \mathrm{L}$ yeast extract) or on LB agar (LB broth substituted with $15 \mathrm{~g} / \mathrm{L}$ agar). Yeast was cultivated in YPD medium (20 g/L glucose, $20 \mathrm{~g} / \mathrm{L}$ peptone and $10 \mathrm{~g} / \mathrm{L}$ yeast extract) or on YPD agar (YPD medium substituted with $18 \mathrm{~g} / \mathrm{L}$ agar). 
Table 11: Bacterial and eukaryotic strains

\begin{tabular}{|c|c|c|}
\hline Strain & Genotype & Company \\
\hline E. coli wt & MG1655 & DSMZ collection \\
\hline E. coli $\mathrm{DH} 5 \alpha$ & 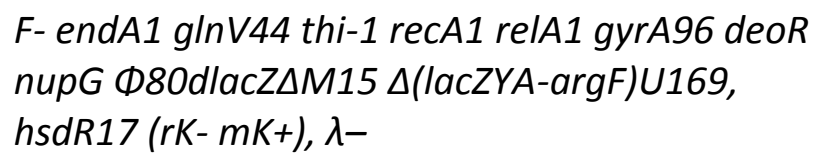 & $\begin{array}{l}\text { New England } \\
\text { Biolabs }\end{array}$ \\
\hline E. coli BL21 (DE3) & $\begin{array}{l}\text { F- ompT gal dcm lon hsdSB( } r B-m B-) \lambda(D E 3 \text { [lacl } \\
\text { lacUV5-T7 gene } 1 \text { ind1 sam7 nin5]) }\end{array}$ & $\begin{array}{l}\text { New England } \\
\text { Biolabs }\end{array}$ \\
\hline E. coli NovaBlue & $\begin{array}{l}\text { endA1 hsdR17 }\left(\mathrm{r}_{\mathrm{K} 12}{ }^{-} \mathrm{m}_{\mathrm{K} 12}^{+}\right) \text {supE44 thi-1 recA1 } \\
\text { gyrA96 relA1 lac } \mathrm{F}^{\prime}\left[\mathrm{proA}^{+} B^{+} \text {lacl }{ }^{q} Z \Delta M 15:: \operatorname{Tn} 10\right] \\
\text { (TetR) }\end{array}$ & Novagen, Merck \\
\hline S. cerevisiae & S288C & $\begin{array}{l}\text { ATCC yeast } \\
\text { collection }\end{array}$ \\
\hline
\end{tabular}

\subsection{Molecular biology procedures}

All primers were purchased from MWG or Eurofins. Kits for plasmid purification or PCR product purification were purchased from Machery Nagel.

\subsubsection{Polymerase chain reaction}

Phusion polymerase ( 2 units) in high-fidelity buffer and dNTPs (0.2 mM each) were used to amplify DNA from a template $\left(0.6 \mathrm{ng}^{\mathrm{l}^{-1}}\right.$ ) (Shampo and Kyle, 2002). The reaction mix was incubated in a thermocycler with an initial DNA denaturing step at $98^{\circ} \mathrm{C}$ for $3 \mathrm{~min}$, followed by 33 cycles of denaturing at $98^{\circ} \mathrm{C}$ for $30 \mathrm{~s}$, annealing at an appropriate temperature for $20 \mathrm{~s}$ and elongation at $72^{\circ} \mathrm{C}$ for $20 \mathrm{~s}$ per kb. For a colony PCR, half an E. coli colony was suspended in $20 \mu$ l water. For the PCR, $2 \mu$ l of the suspension were used as template.

The PCR products were analyzed by agarose-gel electrophoresis and subsequent staining with Serva DNA stain G (Serva). If required, the desired PCR product was purified using either NucleoSpin Gel or PCR Clean-up Machery Nagel kit.

\subsubsection{Site-directed mutagenesis}

The Agilent QuickChange II Site-directed mutagenesis protocol was used to perform site-directed mutagenesis. Primers are listed in supplementary Table 16. 


\subsubsection{Site-directed mutagenesis using isothermal assembly}

An alternative method to perform site-directed mutagenesis was isothermal assembly (Gibson assembly) (Gibson et al., 2009). Two separate PCR products were produced overlapping by $18 \mathrm{nt}$ at each end and together covering the whole plasmid. In both PCRs the desired nucleotide exchange was covered in either the forward primer of PCR 1 or in the reverse primer of PCR 2. The PCR products were purified with the NucleoSpin PCR cleanup kit and quantified photospectrometrically at a wavelength of $260 \mathrm{~nm}$. Isothermal assembly was performed by mixing equal amounts of purified PCR products with T5 exonulcease, Phusion polymerase, Taq ligase, dNTPs and dUTP diphosphatase followed by incubation for $25 \mathrm{~min}$ at $55^{\circ} \mathrm{C}$. In order to remove the original template, the resulting products were treated with Dpnl overnight at $37^{\circ} \mathrm{C}$ to digest methylated DNA. The primers used for Gibson assembly are listed in supplementary Table 16.

\subsubsection{Heat shock transformation}

Chemically competent cells were used for all transformations performed in this study. Cells $(50 \mu \mathrm{l})$ were thawed in a water-ice mixture, $5 \mu$ l DNA solution was added and incubated for $30 \mathrm{~min}$. The transformations were performed by applying a heat shock for $45 \mathrm{~s}$ at $42^{\circ} \mathrm{C}$. Subsequently, the cells were transferred back into the water-ice mixture and allowed to recover for $5 \mathrm{~min}$. Prior to incubation for $60 \mathrm{~min}$ at $190 \mathrm{rpm}$ and $37^{\circ} \mathrm{C}, 700 \mu \mathrm{l}$ LB medium were added. The cell culture was centrifuged for $5 \mathrm{~min}$ at $3000 \mathrm{rpm}$, the supernatant discarded and the cell pellet resuspended in $100 \mu \mathrm{l}$ LB medium. The resusupended cells were subsequently plated on LB agar supplemented with an appropriate selection antibiotic. Colonies were analyzed by colony PCR and Sanger sequencing (Seqlab). Plasmids carrying the desired base-exchange were extracted from the corresponding colony using the NucleoSpin plasmid purification kit (Macherey Nagel).

\subsection{DNA constructs}

The plasmid p00297 (pET28a +) for introducing mutations in EF-P was kindly provided by Christina Kothe (Max Planck Institute for Biophysical Chemistry, MPI BPC). The plasmid encodes EF-P carrying an N-terminal 6xHis tag and its modifying enzymes EpmA, EpmB and EpmC, as well as a kanamycin resistance cassette. The sequences of ribosomal proteins was amplified from genomic DNA of E. coli MG1635 or S. cerevisiae S288C. 


\subsubsection{EF-P labelling variants}

Using the protocol from section 4.7.3 the following EF-P labeling variants were produced (Table 12). Cloning was kindly assisted by Christina Kothe.

Table 12: EF-P labelling variants

\begin{tabular}{ll}
\hline Plasmid & EF-P variants \\
\hline pEF-P T3C & T3C \\
pEF-P M16C & M16C \\
pEF-P S61C & S61C \\
pEF-P D66C (pOF0) & D66C \\
pEF-P A99C & A99C \\
pEF-P I100C & I100C \\
pEF-P N119C & N119C \\
pEF-P E134C & E134C \\
pEF-P S157C & S157C \\
\hline
\end{tabular}

\subsubsection{EF-P functional variants}

To analyze the impact of modification at position K34 in EF-P, the corresponding genes epm $A / B / C$ were deleted from the plasmid pOFO. In addition, to study a potential E-site mRNA codon interaction of EF-P, the following variants were made.

Table 13: EF-P function variants

\begin{tabular}{|c|c|c|c|c|}
\hline Plasmid & Mutation 1 & Mutation 2 & Mutation 3 & Modifying enzymes \\
\hline pOF1 & D66C & D145L & & YjeA, YjeK \& YfcM \\
\hline pOF2 & D66C & D145V & & YjeA, YjeK \& YfcM \\
\hline pOF3 & D66C & T146A & & YjeA, YjeK \& YfcM \\
\hline pOF4 & D66C & T146V & & YjeA, YjeK \& YfcM \\
\hline pOF6 & D66C & Truncation* & & YjeA, YjeK \& YfcM \\
\hline pOF7 & D66C & Truncation* & & YjeA \& YjeK \\
\hline pOF11 & EF-P $w t$ & & & None \\
\hline pOF13 & D66C & & & None \\
\hline pOF14 & D66C & D145L & & None \\
\hline
\end{tabular}




\begin{tabular}{|c|c|c|c|c|}
\hline pOF15 & D66C & D145V & & None \\
\hline pOF16 & D66C & T146A & & None \\
\hline pOF17 & D66C & T146V & & None \\
\hline pOF18 & D66C & Truncation* & & None \\
\hline pOF19 & D66C & $\mathrm{K} 34 \mathrm{~A}$ & & None \\
\hline pOF22 & D66C & D145V & K34A & None \\
\hline pOF23 & D66C & Truncation* & K34A & YjeA, YjeK \& YfcM \\
\hline pOF24 & D66C & K34A & & YjeA \& YjeK \\
\hline pOF25 & D66C & $\mathrm{K} 34 \mathrm{~A}$ & & None \\
\hline pOF26 & D66C & Truncation* & K34A & None \\
\hline pOF27 & D66C & K34A & & None \\
\hline
\end{tabular}

$* \Delta \mathrm{L} 142, \Delta \mathrm{K} 143, \Delta \mathrm{G} 144, \mathrm{D} 145 \mathrm{G}, \mathrm{T} 146 \mathrm{G}, \Delta \mathrm{A} 147, \Delta \mathrm{G} 148, \Delta \mathrm{T} 149$

\subsubsection{EF-P fusion proteins}

For the mass spectrometric analysis of EF-P abundance in vivo (see chapter 2.5), reference proteins were made. The genes encoding the ribosomal proteins L4 and L17 were amplified from E. coli MG1635 genomic DNA and integrated into plasmid pOF13 yielding a L4/17-EF-P fusion protein. The same procedure was carried out for elF5A and the ribosomal protein L4 which were amplified from S. cerevisiae S288C genomic DNA. The following EF-P/elF5A fusion proteins were made (Table 4). The fusion proteins were purified as described in 4.10.1.

Table 14: EF-P fusion proteins

\begin{tabular}{ll}
\hline Plasmid & EF-P fusion protein \\
\hline pOF20 & EF-P-L4 \\
pOF21 & EF-P-L17 \\
pOF30 & elF5A(HYP2)-L4 \\
\hline
\end{tabular}

\subsection{RNA procedures}

\subsubsection{In vitro transcription of tRNA ${ }^{\text {Pro }}$}

The template for in vitro transcription was made by PCR using the plasmid ptRNAPro, which was kindly provided by Dr. Lili Doerfel (MPI BPC). In vitro transcription was 
performed in TRAB (see Table 10) with $10 \mu \mathrm{g} / \mu$ l template, $10 \mathrm{mM}$ DTT, $3 \mathrm{mM}$ NTPs, $5 \mathrm{mM}$ CMP, $0.005 \mathrm{U}^{*} \mu \mathrm{l}^{-1}$ PPase, $0.2 \mathrm{U}^{*} \mu \mathrm{l}^{-1}$ RNAs-inhibitor and $1.6 \mathrm{U}^{*} \mu \mathrm{l}^{-1} \mathrm{T7}$ polymerase (Sampson and Uhlenbeck, 1988) and incubated at $37^{\circ} \mathrm{C}$ for $3 \mathrm{~h}$. The product was purified on a HiTRAP Q HP $5 \mathrm{~mL}$ column in $50 \mathrm{mM} \mathrm{NaOAc} \mathrm{pH} 5,10 \mathrm{mM} \mathrm{MgCl} 2$ applying a gradient up to $1.1 \mathrm{M} \mathrm{NaCl}$. Fractions putatively containing tRNA were further analyzed by $12 \%$ UREA PAGE. RNA was visualized by methylene blue staining. The product containing fractions were pooled and tRNA precipitated. The resulting pellet was dried in a speed-vac pump, resuspended in water and stored at $-80^{\circ} \mathrm{C}$.

\subsubsection{Denaturing polyacrylamide gel electrophoresis}

The transcription products were analyzed under denaturing conditions on a gel containing 12\% acrylamide (acrylamide/bisacrylamide 19:1), 8 M UREA, $100 \mathrm{mM}$ Tris-borate, 2 mM EDTA pH 8.3 and run in TBE buffer (Table 10). Polymerization of acryl/bisacrylamide was catalyzed by the addition of $0.1 \%$ tetramethylenediamine (TEMED) and initiated with $0.005 \%$ ammonium persulfate (APS). Samples were incubated for $2 \mathrm{~min}$ at $95^{\circ} \mathrm{C}$ in loading buffer ( $0.1 \%$ bromphenol, $80 \%$ formamide and $0.1 \%$ xylencyanol in TBE). Prior to loading, the gel was pre-run for $15 \mathrm{~min}$ at $200 \mathrm{~V}$. Samples were loaded and run at $50^{\circ} \mathrm{C}$ at $100 \mathrm{~V}$ until the sample entered the gel, with a subsequent increase to $200 \mathrm{~V}$. The samples were fixed in the gel by incubation in $20 \%$ acetic acid until the bromphenol turns yellow. Staining was performed in a $0.04 \%$ methylene blue in $80 \mathrm{mM} \mathrm{NaOAc} \mathrm{pH} 5$ solution, followed by subsequent destaining in deionized water.

\subsubsection{Precipitation of RNA}

RNA was precipitated by the addition of $1 / 10$ of the volume of $20 \%(w / v)$ potassium acetate $\mathrm{pH} 5.0,2.5$ volumes of ethanol and incubation at $-20^{\circ} \mathrm{C}$ overnight. For the precipitation of low concentrated RNA, ethanol was replaced by one volume of propan-2-ol.

\subsubsection{Aminoacylation of tRNA}

Aminoacylation of tRNA was performed in aminoacylation buffer (Table 10) containing $3 \mathrm{mM}$ ATP and $1 \mathrm{mM}$ DTT. To start the reaction, $1 \mu \mathrm{M}$ prolyl-tRNA synthetase, $0.5 \%$ inorganic pyrophosphatase, $25 \mu \mathrm{M}$ tRNA ${ }^{\text {Pro }}$ and $60 \mu \mathrm{M}\left[{ }^{14} \mathrm{C}\right]$ Pro or $100 \mu \mathrm{M}$ Pro were added and incubated at $37^{\circ} \mathrm{C}$ for $30 \mathrm{~min}$. tRNA was purified by phenol extraction using 2.5 volumes of phenol saturated by addition of $0.3 \mathrm{M}$ potassium acetate (KOAC). After 
rigorous mixing for $30 \mathrm{~s}$, phase separation was facilitated by centrifugation at 4,500 rpm for $5 \mathrm{~min}$ at RT and the aquous phase collected. This procedure was repeated twice. In order to remove phenol traces from the aqueous phase, 2.5 volumes of diethyl ether were added, mixed on a vortex at maximum speed for $30 \mathrm{~s}$, centrifuged at 4,500 rpm for $5 \mathrm{~min}$ at RT and the organic phase removed. This procedure was repeated until no phenol traces at the inter-phase were visible. tRNA was precipitated as described in section 4.9.3 and dissolved in water. Aminoacyl-tRNA was purified on a LithoChrome WP-300 RP18

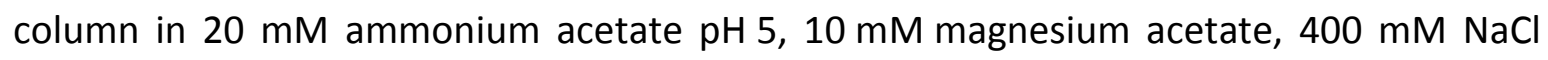
and $5 \%$ ethanol, applying a gradient $0-15 \%$ ethanol over $85 \mathrm{~min}$. Fractions containing isotopically labeled aa-tRNAs were identified by scintillation counting. Non-radioactively labeled aa-tRNAs eluted with the corresponding retention time. Fractions containing aa-tRNA were pooled and precipitated as described 0 , dried in a speed-vac and dissolved in water. Radio isotopic-labeled proline allowed to determine the efficiency of aminoacylation by tRNA precipitation, nitrocellulose filtration and subsequent

${ }^{14} \mathrm{C}$-scintillation counting taking into account the concentration of tRNA determined spectroscopically at $A_{260}$. For aminoacylation with non-radioactive proline the efficiency was determined by the formation of a radio isotopically-labeled tri-peptide. Tritium labeled initiation complexes were used to translate $\mathrm{fMPF}$, in which $\mathrm{f}\left[{ }^{3} \mathrm{H}\right]$ Met and $\left[{ }^{14} \mathrm{C}\right] \mathrm{Phe}$ were used as radioactive markers. The formed product correlates to the Pro-tRNA Pro concentration (as described in 4.13.2). Aminoacylation and concentration determination of other isotopically labeled and unlabeled tRNAs, as well as the determination of the specific activity of ${ }^{14} \mathrm{C}$-Pro was kindly performed by Olaf Geintzer (MPI BPC).

\subsubsection{Synthetic mRNA constructs}

mRNAs were purchased from IBA Life Science. The constructs contain a strong Shine-Dalgarno sequence (G GCA AGG AGG U (Calogero et al., 1988)), which is followed by a linker (AAAUA) and the coding sequence. The chosen codons in this study represent the most abundant tRNA isoacceptors. In order to study the influence of the E-site codon on EF-P binding, in one construct the linker sequence was varied to AACCG. All mRNA constructs are listed in Table 15. 
Table 15: mRNA constructs, with underlined start-codons

\begin{tabular}{|c|c|}
\hline Name & Sequence \\
\hline fMGF & G GCA AGG AGG UAA AUA $\underline{A \cup G}$ GGU UUC \\
\hline fMPFG & G GCA AGG AGG UAA AUA $\underline{A \cup G}$ CCG UUC GGU AUU \\
\hline fMFPG & G GCA AGG AGG UAA AUA $\underline{A \cup G}$ UUC CCG GGU AUU \\
\hline fMPGF & G GCA AGG AGG UAA AUA $\underline{A \cup G}$ CCG GGU UUC AUU \\
\hline fMPPGFV & G GCA AGG AGG UAA AUA $\underline{A \cup G}$ CCG CCG GGU UUC GUU \\
\hline fMPPPF & G GCA AGG AGG UAA AUA $\underline{A \cup G}$ CCG CCG CCG UUC AUU \\
\hline profMFPGI & G GCA AGG AGG UAA CCG $\underline{A \cup G}$ UUC CCG GGU AUU \\
\hline
\end{tabular}

\subsection{Protein purification, labelling and stability}

\subsubsection{Expression and purification of EF-P}

In order to purify EF-P, the corresponding plasmid (To analyze the impact of modification at position $\mathrm{K} 34$ in EF-P, the corresponding genes epmA/B/C were deleted from the plasmid pOFO. In addition, to study a potential E-site mRNA codon interaction of EF-P, the following variants were made.

Table 13) was transformed into E. coli BL21(DE3). A pre-culture was used to inoculate the main culture with a starting optical density $\left(\mathrm{OD}_{600}\right)$ of 0.05 . The cells were cultivated in LB medium supplemented with $0.5 \mu \mathrm{g} / \mathrm{mL}$ kanamycin at $37^{\circ} \mathrm{C}$ at $200 \mathrm{rpm}$ shaking. The overexpression was induced at an $\mathrm{OD}_{600}$ of 0.5 to 0.8 by the addition of $1 \mathrm{mM}$ isopropyl $\beta-(D)$-1-thiogalactopyranoside (IPTG), activating the T7 RNA-polymerase lac promotor. After $3 \mathrm{~h}$ the cells were harvested by centrifugation at 9,600 $\mathrm{xg}$ for $30 \mathrm{~min}$ in a JLA8.1 Beckmann rotor mounted to an Avanti J-26 XP centrifuge (Beckman Coulter). The gained cell pellet was resuspended in $20 \mathrm{mM}$ Tris- $\mathrm{HCl} \mathrm{pH} 8.5,300 \mathrm{mM} \mathrm{NaCl}$, $5 \mathrm{mM}$ 2-mercaptoethanol, and $10 \%$ glycerol (Protino Buffer), supplemented with complete protease inhibitors and DNase. The cells were opened by pressure using an Emulsiflex C-3 homogenizer (Avestin). The cell lysate was centrifuged for $30 \mathrm{~min}$ at $300,000 \times g$ in a JA25.30 Beckmann rotor mounted to an Avanti J-26 XP centrifuge (Beckman Coulter). The supernatant was cleared from insoluble particles by applying it to a $0.45 \mu \mathrm{m}$ glass-fibre filter (Pall Life Science). Because the overexpressed EF-P possesses an $\mathrm{N}$-terminal 6xHis-tag, immobilized metal ion affinity chromatography was used to 
separate EF-P from the cleared lysate. The lysate was applied to a Protino $\mathrm{Ni}$-IDA matrix in a gravity flow column and the manufacturer's protocol was followed. EF-P was eluted in protino buffer supplemented with $250 \mathrm{mM}$ imidazole. The eluate was concentrated using a Vivaspin ${ }^{\circledR} 205 \mathrm{kDa}$ cut-off ultrafiltration unit. The resulting concentrate was further purified and the buffer exchanged by applying the protein solution to a size exclusion chromatography column (Superdex 75 HiLoad 26/60 GE Healthcare) using $\mathrm{TAKM}_{7}$ (see Table 10). The fractions showing an absorbance at $280 \mathrm{~nm}$ were analyzed on a $15 \%$ SDS PAGE. The fractions containing EF-P were pooled and concentrated in a Vivaspin ${ }^{\circledR} 205 \mathrm{kDa}$ cut-off ultrafiltration unit. The concentration of EF-P was determined either by absorbance at $280 \mathrm{~nm}$, assuming a calculated extinction coefficient of $25,440 \mathrm{~cm}^{-1} \mathrm{M}^{-1}$, or by densitometric quantification from the SDS-PAGE using a reference protein of known concentration.

\subsubsection{Expression and purification of EFP-like protein}

In order to purify the EFP-like protein the protocol as described in 4.10 .1 was followed. Two EFP-like protein variants, being either $\mathrm{N}$ - or C-terminally 6xHis-tagged, were produced by Christina Kothe. The concentration of the EFP-like protein was determined by absorbance at $280 \mathrm{~nm}$, assuming a calculated extinction coefficient of $18,910 \mathrm{~cm}^{-1} \mathrm{M}^{-1}$.

\subsubsection{Sodium dodecyl sulfate polyacrylamide gel electrophorese (SDS-PAGE)}

Protein purity was analyzed by SDS PAGE (Laemmli, 1970; Weber et al., 1972). The standard resolving gel contained $15 \%$ acrylamide (29:1 acryamide/bisacrylamide), $40 \mathrm{mM}$ Tris- $\mathrm{HCl} \mathrm{pH} 8.8$ at RT and 0.1\% SDS, whereas the standard stacking gel consisted of $4 \%$ acrylamide (29:1 acryamide/bisacrylamide), $12,5 \mathrm{mM}$ Tris- $\mathrm{Hcl} \mathrm{pH} 6.8$ at RT and $0.1 \%$ SDS. Polymerization of the gel solution was induced by the addition of $0.05 \%$ APS and catalyzed by $0.1 \%$ TEMED. To analyze the ribosomal protein L33, the concentration of Tris- $\mathrm{HCl}$ was doubled and acrylamide (29:1 acryamide/bisacrylamide) was increased to $18 \%$. Proteins were visualized by incubating the gel for $10 \mathrm{~min}$ in Coomassie staining solution (Table 10) and subsequent destaining with destaining solution (Table 10).

For mass-spectrometric analysis, pre-casted Mini-PROTEAN ${ }^{\circledR}$ TGX $^{\mathrm{TM}}$ BioRad gels were used. All SDS poly-acrylamide gels were run vertically in a BioRad gel system. 


\subsubsection{Labeling of EF-P}

Prior to labeling, EF-P was reduced with a 10-fold molar excess of DTT or TCEP for $30 \mathrm{~min}$ at RT shaking with $20 \mathrm{rpm}$. To avoid that the thio-reactive dyes reacts with the excess of DTT, the buffer was changed to TK buffer (see Table 10) using a G25 midi column (GE Healthcare) following the manufacturer's spin protocol. TCEP is mostly unreactive and therefore is not required to be removed from the buffer. For dyes functionalized with a maleimide group, TK pH 8.3 and for iodacetamide functionalized dyes, TK pH 7.3 was used. The dyes were solved in dimethylformamide (DMF) to yield a final concentration of $10 \mathrm{mM}$. While EF-P $(100 \mu \mathrm{M})$ solution was mixed on a Vortex (Scientific Industries), the dye (final concentration $1 \mathrm{mM}$ ) was added dropwise and the reaction mix was incubated at room temperature protected from light for $2 \mathrm{~h}$. The reaction was quenched by the addition of $10 \mathrm{mM}$ 2-mercaptoethanol and centrifuged at 16,000 x g for $5 \mathrm{~min}$ in a bench top centrifuge (5415R Eppendorf). Free dye was removed by seize exclusion chromatography using either G25 midi columns following the gravity flow protocol or on a Superdex S75 10/300 GL (GE Healthcare) column. Fractions containing labeled proteins were analyzed spectrophotometrically at a wavelength of $280 \mathrm{~nm}$ and the corresponding maximum absorbance of the dye used. AleaxaFluor ${ }^{\mathrm{TM}} 488$ (AF488) has the maximum absorption $\left(\lambda_{\max }\right)$ at $495 \mathrm{~nm}$ and an extinction coefficient of $\varepsilon=73,000 \mathrm{~cm}^{-1} * \mathrm{M}^{-1}$. QSY-7 has the maximum absorption at $560 \mathrm{~nm}$ and an extinction coefficient of $\varepsilon=90,000 \mathrm{~cm}^{-1} * \mathrm{M}^{-1}$. QSY-9 has the maximum absorption at $562 \mathrm{~nm}$ and an extinction coefficient of $\varepsilon=88,000 \mathrm{~cm}^{-1} * \mathrm{M}^{-1}$. QSY-35 has its absorbance maximum at $475 \mathrm{~nm}$ and an extinction coefficient of $\varepsilon=23,000 \mathrm{~cm}^{-1} * \mathrm{M}^{-1}$. Atto540Q has its maximum absorption at $543 \mathrm{~nm}$ and an extinction coefficient of $\varepsilon=105,000 \mathrm{~cm}^{-1} * \mathrm{M}^{-1}$.

The degree of labeling (DOL) was calculated as described in Equation 1.

Equation 1: Calculation of the degree of labeling.

$D O L=\frac{A_{\max } / \varepsilon_{\max }}{A_{\text {Prot }} / \varepsilon_{\text {Prot }}}$

$A_{\max }$ is defined as the absorbance of the dye at $\lambda_{\max } . \varepsilon_{\max }$ is defined as the extinction coefficient of the dye at the absorbance maximum. $\varepsilon_{\text {Prot }}$ is defined as the extinction coefficient at the absorption maximum of the dye. Aprot is defined as described in Equation 2, and $\mathrm{CF}_{280}$ is defined as described in Equation 3. 
Equation 2: Calculation of the $A_{\text {prot }}$.

$A_{\text {Prot }}=A_{280}-\left(A_{\max } * C F_{280}\right)$

Equation 3: Calculation of the correction factor $\mathrm{CF}_{280}$.

$C F_{280}=\frac{\varepsilon_{280}}{\varepsilon_{\max }}$

The fractions with the highest DOL were pooled and used for this study.

\subsubsection{ProteoPlex analysis}

In order to investigate whether the introduced point mutations had an effect on the stability of EF-P, the commercially available ProteoPlex system was used (Chari et al., 2015). Protein solution $(18 \mu \mathrm{l})$ with a concentration of $5 \mathrm{mg} / \mathrm{ml}$ was mixed with $2 \mu \mathrm{l}$ of SYPRO $^{\circledR}$ Orange Protein Gel stain solution in a 96-well microtiter plate (Biozym) and applied to the ProteoPlex reader. As reference, four replicates of EF-P (wt) protein and two blanks of TAKM7 buffer and de-ionized water were used.

\subsubsection{Purification of the ribosomal protein L33}

The plasmid pET24A rpmG P31C encoding for the labeling variant of the ribosomal protein L33 (P31C) was kindly provided by Dr. Belardinelli (MPI BPC). The plasmid was transformed into E. coli BL21(DE3) and the protein expressed as described in chapter 4.10.1. The cell lysate was centrifuged for $30 \mathrm{~min}$ at 300,000 x g in a JA25.30 Beckmann rotor mounted to an Avanti J-26 XP centrifuge (Beckman Coulter). The pellet was washed, dissolved and flash frozen in opening buffer (5 mM HEPES pH7.5, $200 \mathrm{mM} \mathrm{KCl,5 \%} \mathrm{glycerol}$ and complete protease inhibitor). For cell lysis, the cells were thawed and applied to a Digital sonifier cell disruptor W-250D (Branson) using 8 pulses with an amplitude of $20 \%$ with a period of $20 \mathrm{~s}$. The resulting cell lysate was centrifuged at 30,000 x g for $30 \mathrm{~min}$, the supernatant was discarded since it mainly contains the undesired L33 wt protein. The desired L33 labeling variant was mainly found in the inclusion bodies. The pellet containing membrane and inclusion bodies was resuspended and stirred for $1 \mathrm{~h}$ at RT in $50 \mathrm{mM}$ HEPES pH 7.5, $50 \mathrm{mM} \mathrm{KCl}, 5 \%$ glycerol, $5 \mathrm{mM}$ 2-mercaptoethanol and $6 \mathrm{M}$ urea and further homogenized using a Dounce tissue grinder (Wheaton). The solution was centrifuged at $30,000 \times \mathrm{g}$ for $30 \mathrm{~min}$. The supernatant was diluted 10 -fold with $50 \mathrm{mM}$ HEPES pH 7.5, 5\% glycerol, $5 \mathrm{mM}$ 2-mercaptoethanol and $6 \mathrm{M}$ urea and applied to 
a $1 \mathrm{~mL}$ HiTrap SP HP cation exchange chromatography column (GE Lifescience). The separation of proteins was performed in $50 \mathrm{mM}$ HEPES $\mathrm{pH} 7.5,5 \%$ glycerol, $5 \mathrm{mM}$ 2-mercaptoethanol and $6 \mathrm{M}$ urea with a gradient of $5 \mathrm{mM}$ to $300 \mathrm{mM} \mathrm{KCl}$ over 30 column volumes (CV). The fractions were analyzed by SDS-PAGE as described in chapter 0 . Fractions containing L33 were pooled, diluted 10-fold and applied to a Resource S cation exchange column (GE Lifescience). The separation of proteins was performed in $50 \mathrm{mM}$ HEPES $\mathrm{pH}$ 7.5, 5\% glycerol, $5 \mathrm{mM}$ 2-mercaptoethanol and $6 \mathrm{M}$ urea with a gradient of $5 \mathrm{mM}$ to $300 \mathrm{mM} \mathrm{KCl}$ over 30 column volumes (CV). Fractions containing L33 were pooled, flash frozen and stored at $-80^{\circ} \mathrm{C}$.

\subsubsection{Labeling and refolding of ribosomal protein L33}

Labeling of L33 was performed under denaturing conditions in the presents of $6 \mathrm{M}$ urea. The concentration of L33 was adjusted to $100 \mu \mathrm{M}$ and the protein was incubated with $10 \mathrm{mM}$ AlexaFluor488 (AF488) dissolved in DMF at RT in the dark for 90 min while rotating at $28 \mathrm{rpm}$. The reaction was quenched by the addition of a 10-fold excess of L33 quenching buffer $(25 \mathrm{mM} \mathrm{HEPES} \mathrm{pH} \mathrm{7.3,30} \mathrm{mM} \mathrm{KCl,} 6 \mathrm{mM}$ 2-mercaptoethanol, $10 \%$ glycerol and $6 \mathrm{M}$ urea). In order to separate the labeled protein from free dye, the mixture was manually applied to a $1 \mathrm{ml}$ HiTrap HP SP column. The flow-through was reapplied to the column. The free dye was removed by washing the column with $10 \mathrm{CV}$ of quenching buffer. The L33-AF488 conjugate was eluted using L33 quenching buffer with $300 \mathrm{mM} \mathrm{KCl}$. In order to refold the L33-AF488 conjugate, the eluate was applied to multiple step dialysis. Using a $3.5 \mathrm{kDa}$ cut-off midi D-tube dialyzer (Merck Millipore), the buffer was exchanged to 133 storage buffer $(25 \mathrm{mMHEPES}, 300 \mathrm{mM} \mathrm{KCl}$ and $10 \%$ glycerol). In the first dialysis step the urea concentration was reduced to $4 \mathrm{M}$ urea ( $4 \mathrm{~h}$ of incubation at RT). In the second step, the urea concentration was reduced to $2 \mathrm{M}$ urea $\left(4 \mathrm{~h}\right.$ at $\left.4^{\circ} \mathrm{C}\right)$. In the third step, no urea was present in the buffer $\left(\mathrm{o} / \mathrm{n}\right.$ at $\left.4^{\circ} \mathrm{C}\right)$. The refolded L33-AF488 conjugate was flash frozen and stored at $-80^{\circ} \mathrm{C}$.

\subsection{Ribosome complexes and translation}

\subsubsection{Reconstitution of 50S subunits with the ribosomal protein L33}

Large ribosomal subunits lacking L33 were prepared according to (Peske et al., 2005; Rodnina and Wintermeyer, 1995) and kindly provided by Sandra Kappler (MPI BPC). Reconstitution using L33 labeled with AF488 was carried out using a 1.05 excess of L33 
over $50 \mathrm{~S}$ subunits in $50 \mathrm{mM}$ HEPES $\mathrm{pH} 7.5,400 \mathrm{mM} \mathrm{KCl}, 20 \mathrm{mM} \mathrm{MgCl}$, $6 \mathrm{mM} 2$-mercaptoethanol and $10 \%$ glycerol for $1 \mathrm{~h}$ at $37^{\circ} \mathrm{C}$ (Belardinelli et al., 2016a). The sample was cooled on ice and centrifuged for $5 \mathrm{~min}$ at $16,000 \times \mathrm{g}$ at $4^{\circ} \mathrm{C}$. To remove the excess of L33-AF488 the supernatant was transferred on a $30 \%$ sucrose cushion in $\mathrm{TAKM}_{7}$ buffer, and centrifuged in an MLA-130 rotor for $2 \mathrm{~h}$ at $4^{\circ} \mathrm{C}$ at 385,900 $\mathrm{xg}$. The supernatant was discarded and the centrifugation tube placed on ice. $\mathrm{TAKM}_{7}$ buffer was added onto the pellet and incubated for $10 \mathrm{~min}$ on ice. The pellet was dissolved by carefully tapping against the tube. The solution was centrifuged for $2 \mathrm{~min}$ with $16,000 \times \mathrm{g}$ at $4^{\circ} \mathrm{C}$. The reconstituted 50 S subunits were quantified photometrically at a wavelength of $260 \mathrm{~nm}$ $\left(1 \mathrm{OD}_{260}=37 \mathrm{pmol}\right)$ and $495 \mathrm{~nm}\left(\varepsilon=73,000 \mathrm{~cm}^{-1} * \mathrm{M}^{-1}\right)$; the ratio reflects the reconstitution efficiency.

\subsubsection{Assembly of 70S initiation and post-translocation complexes}

The assembly and purification of 705 initiation and post-translocation complexes was performed as described by Rodnina and co-workers (Belardinelli et al., 2016a; Rodnina et al., 1997). $30 \mathrm{~S}$ subunits were activated by incubation in $\mathrm{TAKM}_{20}$ buffer for $30 \mathrm{~min}$ at $37^{\circ} \mathrm{C}$. In order to assemble $1 \mu \mathrm{M}$ 70S initiation complex, $1.5 \mu \mathrm{M}$ 30S subunits were incubated with $3 \mu \mathrm{M}$ mRNA, $2 \mu \mathrm{M}$ IF1, IF2 and IF3, $2.5 \mu \mathrm{M}$ f $\left[{ }^{3} \mathrm{H}\right]$ Met-/fMet-tRNA ${ }^{\mathrm{fMet}}$ and $1 \mu \mathrm{M} 50 \mathrm{~S}$ subunits in either $\mathrm{TAKM}_{7}$ or HiFi buffer containing $1 \mathrm{mM} \mathrm{GTP}$ for $30 \mathrm{~min}$ at $37^{\circ} \mathrm{C}$. Subsequently, the reaction mix was cooled on ice for $5 \mathrm{~min}$ and centrifuged for $2 \mathrm{~min}$ at $16,000 \times \mathrm{g}$. The supernatant was applied to a $30 \%$ sucrose cushion in either $\mathrm{TAKM}_{20}$ or $\mathrm{HiFi}$ and centrifuged for $2 \mathrm{~h}$ at $4^{\circ} \mathrm{C}$ at $259,000 \times \mathrm{g}$ using a TLS-55 rotor. The pellet was resuspended in $\mathrm{TAKM}_{7}$ and the ribosome complexes quantified spectrophotometrically at a wavelengths of $260 \mathrm{~nm}\left(1 \mathrm{OD}_{260}=23 \mathrm{pmol}\right)$. If applicable, initiation was quantified by nitrocellulose filtration and subsequent scintillation counting. Complexes that contained non-radioactive $\mathrm{fMet} \mathrm{tRNA} \mathrm{fMet}^{\mathrm{fM}}$ were quantified by forming a radioactively labeled di-peptide.

Post-translocations complexes were formed by the addition of the corresponding ternary complex in a two-fold excess over the codon concentration and incubation for $2 \mathrm{~min}$ at $37^{\circ} \mathrm{C}$. Ternary complexes were prepared by incubating the corresponding aminoacyl-tRNA with a 2-fold (in the case of proline 3-fold) molar excess of EF-Tu, $1 \mathrm{mM}$ GTP, $3 \mathrm{mM} \mathrm{PEP}$, and $0.1 \mathrm{mg}^{*} \mathrm{ml}^{-1}$ pyruvate kinase for $15 \mathrm{~min}$ at $37^{\circ} \mathrm{C}$. The formed complexes were treated as described above. Initiation and post-translocation complexes 
were also formed using purified $70 \mathrm{~S}$ subunits kindly provided by Sandra Kappler. The formation of complexes was performed as described without prior ribosome activation step.

\subsection{Binding kinetics of EF-P}

All kinetic studies were performed at $37^{\circ} \mathrm{C}$ in HiFi buffer.

\subsubsection{Rapid kinetics of EF-P binding}

Rapid kinetic experiments of EF-P binding to the ribosome were performed in a SX-20MV stopped-flow apparatus (Applied Biophysics), which allows the rapid mixing of two solutions while monitoring fluorescence.

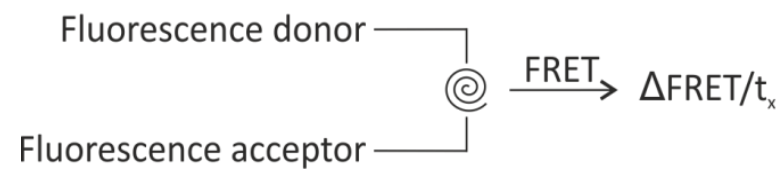

Figure 27: Schematic depiction of the stopped-flow setup used in this study A stopped-flow apparatus allows to rapidly mix two solutions while acquiring optical changes in real time. The device has an approximate dead time of $1.5 \mathrm{~ms}$ and can be operated under temperature controlled conditions. After loading the fluorescent solutions into the corresponding syringes equal volumes are mixed and pass through an optical chamber until the flow is stopped by the stop-syringe. Depending on the used detectors FRET, anisotropy or light scattering can be monitored. In this study fluorescence changes resulting from FRET were monitored.

To measure EF-P binding, ribosome complexes harboring L33-AF488 were excited at a wavelength of $470 \mathrm{~nm}$ and the emitted light was monitored after passing through a $500 \mathrm{~nm}$ cut-off filter (KV-500). EF-P QSY-35 quenches the fluorescence of L33-AF488 containing ribosome complexes upon coming in close proximity. Fluorescent ribosome complexes were used at a final concentration of $50 \mathrm{nM}$ and were mixed with 0.25 - $3 \mu \mathrm{M}$ EF-P(QSY). Samples were prepared in $600-800 \mu \mathrm{l}$ of HiFi buffer. Prior to loading, the samples were centrifuged for $2 \mathrm{~min}$ at $16,000 \mathrm{rcf}$ at $4^{\circ} \mathrm{C}$. One measurement required $60 \mu \mathrm{l}$ of each sample and 7 to 10 technical replicates were acquired. From each rapid mixing experiment 5,000 logarithmically distributed data points were acquired. Each time course experiment was plotted in GraphPad Prism. The data was analyzed by two-exponential (Equation 4) with an additional term for a linear slope if needed (Equation 5) using TableCurve 2D v5.01.01 (Systat Software Inc.). 
Equation 4: Two-exponential function

$$
f(X)=A+B * \operatorname{EXP}(-C * X)+D * \operatorname{EXP}(-E * X)
$$

Equation 5: Two-exponential function with the addition of a slope

$$
\mathrm{f}(\mathrm{X})=\mathrm{A}+\mathrm{B} * \operatorname{EXP}(-\mathrm{C} * \mathrm{X})+\mathrm{D} * \operatorname{EXP}(-\mathrm{E} * \mathrm{X})+\mathrm{F} * \mathrm{X}
$$

\subsubsection{Equilibrium kinetics of EF-P binding}

To analyze the binding affinity of EF-P for different substrates, an equilibrium EF-P titration was performed. Fluorescent ribosome complexes were titrated with EF-P QSY-35 in a spectrofluorimeter. EF-P was added to $10 \mathrm{nmol}$ ribosome complexes, mixed and incubated for $30 \mathrm{~s}$ at $37^{\circ} \mathrm{C}$ to reach equilibrium. Fluorescence was excited at $470 \mathrm{~nm}$ and the fluorescence emission was monitored at $530 \pm 5 \mathrm{~nm}$ using a monochromator. The data points were fitted using a quadratic function (Equation 6) in GraphPad Prism.

Equation 6: Quadratic function

$$
f(X)=A+B *[(I C+X+K d)-\operatorname{sqrt}(\operatorname{sqr}(I C+X+K d)-4 * I C * X)]
$$

\subsubsection{Equilibrium kinetics using anisotropy}

For non-fluorescent ribosome complexes, the anisotropy of AlexaFluor 488 conjugated to EF-P was used to study the dissociation constants. $10 \mathrm{nmol}$ of EF-P-AF488 were mixed with an increasing concentration of ribosome complexes and equilibrated for $30 \mathrm{~s}$ at $37^{\circ} \mathrm{C}$. The anisotropy values were corrected for light scattering caused by the ribosome. The following equation was used to calculate the anisotropy:

Equation 7: Calculation of anisotropy

$$
r=(\mathrm{vv}-(\mathrm{hv} / \mathrm{hh}) * \mathrm{vh}) /(\mathrm{vv}+2 *(\mathrm{hv} / \mathrm{hh}) * \mathrm{vh})
$$

Where (hv/hh) is an empirical correction factor accounting for a device- and wavelength-dependent polarization detection bias; vv and vh represents the measured intensities derived from the vertical excitation; hh and hv represent the measured intensities derived from the horizontal excitation.

\subsection{Kinetics of EF-P facilitated catalysis}

All experiments aimed at the role of EF-P in facilitating peptide bond formation were performed in HiFi buffer, if not stated elsewise. Time-resolved experiments were performed, as applicable, manually or using a quench-flow apparatus (Figure 28). 


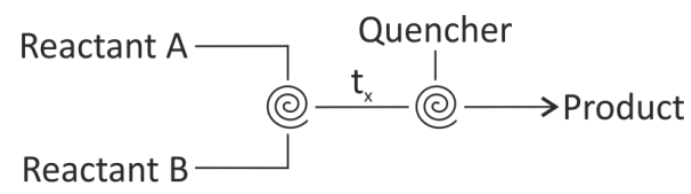

Figure 28: Schematic depiction of a quenched-flow apparatus.

A quenched-flow apparatus allows to rapidly mix two reactants and subsequently quench the reaction at desired times. First, equal volumes of the two reactants are mixed and allowed to react for a variable time $\left(t_{x}\right)$ at a given temperature. Then, a quencher is added, to stop the reaction. The dead time of the machine is approximately $2 \mathrm{~ms}$.

After quenching, the neutralized reaction mixes were loaded on a Chromolith ${ }^{\circledR}$ Performance RP8e 100-4.6 mm column using a Walters HPLC unit 2695. Separation of products and educts was achieved with a 0 to $65 \%$ acetonitrile gradient (depicted in Figure 29) in 0.1\% TFA. Product yields were assessed via double-scintillation counting, plotted and fitted using a two-phase association model.

\subsubsection{Time-resolved puromycin reaction}

When puromycin was used as analog of the A-site substrate, a time-resolved puromycin reaction (Holtkamp et al., 2004) was performed. Purified initiation complexes were mixed with Pmn and sub-saturating concentrations of EF-P in a quench-flow apparatus. The reaction was quenched with $50 \%$ formic acid and ribosome complexes dissociated by adding $500 \mu \mathrm{l} 1.5 \mathrm{M}$ sodium acetate saturated with $\mathrm{MgSO}_{4}$. The formed products were extracted using $750 \mu$ l ethyl acetate. The mixture was shaken with $250 \mathrm{rpm}$ for $30 \mathrm{~min}$. Phase separation was facilitated by centrifugation at $16,000 \times \mathrm{g}$ for $1 \mathrm{~min}$ at RT. The extracted products were applied to $3 \mathrm{H}$ scintillation counting.

\subsubsection{Tri-peptide formation}

In order to quantify non-radioactive Pro-tRNA ${ }^{\text {Pro }}$, initiation complexes $(0.15 \mu \mathrm{M})$ primed with fMPF mRNA in the presence of $2 \mu \mathrm{M}\left[{ }^{14} \mathrm{C}\right] \mathrm{Phe}-\mathrm{TC}, 1 \mu \mathrm{M}$ EF-G and $3 \mu \mathrm{M}$ EF-P were titrated with increasing concentrations of Pro-tRNA ${ }^{\text {Pro }}$. The reaction was allowed to proceed at $37^{\circ} \mathrm{C}$ for $1 \mathrm{~min}$ and quenched with $\mathrm{KOH}(0.5 \mathrm{mM})$. The peptidyl-tRNA was hydrolyzed by incubation at $37^{\circ} \mathrm{C}$ for 30 min. Prior to applying the samples to an HPLC system, the reaction mix was centrifuged at $16,000 \mathrm{rcf}$ for $15 \mathrm{~min}$ at $4^{\circ} \mathrm{C}$. The supernatant was diluted with HPLC buffer A (Table 10) to a final volume of $200 \mu$ and applied via a HPLC unit 2695 (Waters) to a Chromolith ${ }^{\circledR}$ Performance RP-8e reversed phase column. 
The eluate was fractionated in $0.5 \mathrm{ml}$ fractions, applied to double scintillation counting and the yield plotted. The incorporation of cold Pro is required to form the tri-peptide product. Hence, the ${ }^{14} \mathrm{C}$ counts reflect the proline concentration.

\subsubsection{Penta-peptide formation}

In vitro translation was performed by mixing initiation complex $(0.2 \mu \mathrm{M})$ primed with an mRNA coding for fMPPPF, Pro- and Phe-TC (2 $\mu \mathrm{M}$ each), EF-G $(1 \mu \mathrm{M})$ and EF-P (varying concentrations) in $\mathrm{HiFi}$ buffer at $37^{\circ} \mathrm{C}$. The reaction was allowed to proceed for $20 \mathrm{~s}$ and subsequently quenched with $\mathrm{KOH}(0.5 \mathrm{M})$. The product was hydrolyzed for $30 \mathrm{~min}$ at $37^{\circ} \mathrm{C}$ and neutralized with glacial acetic acid. The reaction mix was centrifuged at 16,000 rcf for $15 \mathrm{~min}$ at $4^{\circ} \mathrm{C}$. The product was separated from the substrates by reversed-phase HPLC using a Chromolith ${ }^{\circledR}$ Performance RP 8e reversed phase column. The eluate was fractionated in $0.5 \mathrm{ml}$ fractions and applied to double scintillation counting.

To monitor the formation of fMPPG from fMPP post-translocation complexes, fMPP complexes $(0.1 \mu \mathrm{M})$, saturating concentrations of Gly-TC $(10 \mu \mathrm{M})$ and sub-saturation concentrations of EF-P were rapidly mixed and incubated in $\mathrm{HiFi}$ for $37^{\circ} \mathrm{C}$. The reaction mix was centrifuged at $16,000 \mathrm{rcf}$ for $15 \mathrm{~min}$ at $4^{\circ} \mathrm{C}$. The product was separated from the substrates by reversed-phase HPLC using a Chromolith ${ }^{\circledR}$ Performance RP 8e column. The eluate was fractionated in $0.5 \mathrm{ml}$ fractions and applied to double scintillation counting.

\subsubsection{Peptidyl-tRNA drop off}

In order to study the peptidyl-tRNA drop off during translation, the amount of peptidyltRNA bound to the ribosome was quantified. For this purpose, initiation complexes $(0.3 \mu \mathrm{M})$ primed with an mRNA encoding fMPPG were incubated with Pro-TC $(4 \mu \mathrm{M})$, Gly-TC $(2 \mu \mathrm{M}), \mathrm{EF}-\mathrm{G}(3 \mu \mathrm{M})$ in the presence or absence of either EF-P $(3 \mu \mathrm{M})$ or EFP-like protein $(3 \mu \mathrm{M})$ in $\mathrm{HiFi}$ at $37^{\circ} \mathrm{C}$ for $120 \mathrm{~s}$. The reaction mix was applied on a $0.2 \mu \mathrm{m}$ nitrocellulose filter (Sartorius), allowing free tRNAs to path through, while tRNAs bound to the ribosome were retained. In order to reduce unspecific binding of free tRNAs the filter were washed with $5 \mathrm{ml}$ ice cold HiFi buffer. The retained tRNAs were quantified by scintillation counting. 


\subsubsection{Pulse-chase experiment}

In order to gain mechanistic insights into how EF-P facilitates peptide bond formation a pulse-chase experiment was performed. The amplitude of the reaction reflects the commitment of EF-P to perform catalysis over dissociation and will give insights whether binding leads immediately to catalysis or requires multiple rounds of binding. Post-translocation complexes (FMPP) primed with mRNA encoding FMPPG were used and the incorporation of glycine was monitored. The experiments were performed in a quench-flow apparatus using $\mathrm{HiFi}$ buffer at $37^{\circ} \mathrm{C}$. The pulse-chase was performed by rapidly mixing of post-translocation complexes $(0.4 \mu \mathrm{M})$ and EF-P $(2 \mu \mathrm{M})$ with Gly-TC $(6.6 \mu \mathrm{M})$ and EF-P (K34A) $(20 \mu \mathrm{M})$. To establish the potential amplitude of the pulse chase experiment the reaction was monitored in the absence of unreactive EF-P variants (maximum amplitude) and with unreactive EF-P in the start mix (minimum amplitude). The chase was measured in the presence of both the reactive and unreactive EF-P in the start mix. The maximum amplitude was determined by rapidly mixing of post-translocation complexes $(0.4 \mu \mathrm{M})$ and $\mathrm{EF}-\mathrm{P}(\mathrm{K} 34 \mathrm{~A})(10 \mu \mathrm{M})$ with Gly-TC $(6.6 \mu \mathrm{M})$ and EF-P K34A $(10 \mu \mathrm{M})$. The minimum amplitude was determined by rapidly mixing of post-translocation complexes $(0.4 \mu \mathrm{M})$ and EF-P $(1 \mu \mathrm{M})$ with Gly-TC $(6.6 \mu \mathrm{M})$ and EF-P $(1 \mu \mathrm{M})$. The chase was carried out by rapidly mixing of posttranslocation complexes $(0.4 \mu \mathrm{M})$, EF-P $(2 \mu \mathrm{M})$ and EF-P K34A $(20 \mu \mathrm{M})$ with Gly-TC $(6.6 \mu \mathrm{M})$. The reactions were quenched after 0.02, 2 and $20 \mathrm{~s}$ with $\mathrm{KOH}(0.5 \mathrm{M})$. The products were hydrolyzed for $30 \mathrm{~min}$ at $37^{\circ} \mathrm{C}$ and neutralized with glacial acetic acid. The reaction mix was centrifuged at $16,000 \mathrm{rcf}$ for $15 \mathrm{~min}$ at $4^{\circ} \mathrm{C}$. The product was separated from the substrates by reversed-phase HPLC using a Chromolith ${ }^{\circledR}$ Performance $\mathrm{RP} 8 \mathrm{e}$ column. The eluate was fractionated in $0.5 \mathrm{ml}$ fractions and applied to double scintillation counting.

\subsection{Mass-spectrometry}

\subsubsection{Sample preparation for mass spectrometry}

In order to quantify the cellular concentration of EF-P in E. coli cell samples were prepared. The cells were cultured in either LB broth at $37^{\circ} \mathrm{C}$ shaking with $200 \mathrm{rpm}$, in a 21 glass flask containing $500 \mathrm{ml}$ medium. One $\mathrm{OD}_{600}$ of cells was harvested during their exponential growth phase at an $\mathrm{OD}_{600}$ of 0.5 by centrifugation with $16,000 \mathrm{x}$ g for 5 min. 
The cell pellets were dissolved in SDS sample buffer and denatured at $95^{\circ} \mathrm{C}$ for $5 \mathrm{~min}$. The samples corresponding to a $0.1 \mathrm{OD}_{600}$ of cells were applied to an SDS PAGE and allowed to enter the separation gel for $1 \mathrm{~cm}$. In order to establish correct factors that account for differences in peptide ionizability, EF-P fusion proteins were created. The proteins were digested in gel as described in the following section (4.14.2).

\subsubsection{In-gel digest and peptide extraction}

Prior to the in-gel digestion, the gel strips containing the protein of interest were cut out of the gel, crushed, and washed with water for $5 \mathrm{~min}$ at RT. After removal of the liquids, the gel pieces were shrunk by applying $150 \mu \mathrm{l}$ acetonitrile and incubation for $15 \mathrm{~min}$ shaking with $150 \mathrm{rpm}$ at RT. The supernatant was removed, and the gel pieces dried in a speed-vac. The proteins inside the gel pieces were reduced with $10 \mathrm{mM}$ DTT (in $50 \mathrm{mM}$ ammonium hydrogen carbonate) at $56^{\circ} \mathrm{C}$ shaking with $150 \mathrm{rpm}$ for $60 \mathrm{~min}$. The gel pieces were again shrunken using acetonitrile. The reduced cysteine moieties were conjugated to iodoacetamide by using $55 \mathrm{mM}$ iodoacetamide in $50 \mathrm{mM}$ ammonium hydrogen carbonate. The gel pieces were washed with $50 \mathrm{mM}$ ammonium hydrogen carbonate and twice dehydrated with acetonitrile prior to in-gel digestion.

The gel pieces were rehydrated with digestion buffer on ice for $45 \mathrm{~min}$. The digestion buffer contained trpysin $(12.5 \mathrm{ng} / \mu \mathrm{l})$ solved in $50 \mathrm{mM}$ ammonium hydrogen carbonate and $0.5 \mathrm{mM}$ calcium chloride solution. The digestion was performed overnight at $37^{\circ} \mathrm{C}$. To avoid dehydration the gel pieces covered with digestion buffer lacking the corresponding protease.

The peptides were extracted by adding $5 \%$ formic acid and acetonitrile. The samples were dried in a speed-vac and the peptides were dissolved in $10 \%$ acetonitrile and $0.1 \%$ formic acid solution.

\subsubsection{Quantification of intracellular EF-P by mass spectrometry}

The proteolyzed peptides were analyzed by a UPLC-ESI-MS/MS system (Dionex Ultimate 3000 UPLC connected to a QExcative Plus mass spectrometer) and an untargeted data-dependent (DDA) acquisition was performed. RAW files were analyzed using the MaxQuant software (version1.5.2.8) and searched against the UniProt E. coli (K12) proteome as reference. Results were analyzed in the Skyline software. The highest populated charge states of most intense peptides with suitable elution profiles were 
selected for targeted acquisition. Those peptides were quantified in the proteolyzed fusionproteins and the E.coli lysate. Precusors were isolated in a $1 \mathrm{~m} / \mathrm{z}$ isolation window, in a maximum fill time of $100 \mathrm{~ms}$ and an automatic gain of $5 \times 10^{5}$. Precusor peptides were fragmented by HCD at a collsion energy of $30 \mathrm{eV}$ and detected at set resolution of 60000 in the orbitrap. Fragment elution profiles of the individual peptides were extracted and integrated inSkyline software 3.5. The integrated area of each fusion-protein derived EF-P or L4/L17 peptide was used to form their ratio which was corrected by the established correction factors yielding a corrected EF-P-ribosome ratio. 


\section{Appendix}

\subsection{Abbreviations}

\begin{tabular}{|c|c|}
\hline$\mu \mathrm{M}$ & Micromolar \\
\hline$A_{260}$ & Absorbance at $260 \mathrm{~nm}$ \\
\hline aа & Amino acid \\
\hline aa-tRNA & Aminoacyl-tRNA \\
\hline aa-RS & Aminoacyl-tRNA synthetases \\
\hline alF5A & Archaeal initiation factor $5 \mathrm{~A}$ \\
\hline A site & Aminoacyl site \\
\hline bp & Base pair \\
\hline DDA & Data dependent acquisition \\
\hline EF & Elongation factors (EFs), \\
\hline EF-G & Elongation factor $\mathrm{G}$ \\
\hline EF-Tu & Elongation factor Tu \\
\hline EF-P & Elongation factor $\mathrm{P}$ \\
\hline elF5A & Eukaryotic initiation factor $5 \mathrm{~A}$ \\
\hline E site & Exit site \\
\hline FRET & Fluorescence resonance energy transfer \\
\hline g & Relative centrifugal force \\
\hline gm & Gram \\
\hline GTP & Guanosine triphosphate \\
\hline IC & Initiation complex \\
\hline IF & Initiation factors \\
\hline $\mathrm{k}_{\mathrm{app}}$ & Apparent rate constant \\
\hline$K_{d}$ & Equilibrium dissociation constant \\
\hline $\mathrm{kDa}$ & Kilodalton \\
\hline$k_{\text {off }}$ & Dissociation rate constant \\
\hline $\mathrm{k}_{\text {on }}$ & Association rate constant \\
\hline $\mathrm{MDa}$ & Mega Dalton \\
\hline MDS & Molecular dynamics simulations \\
\hline $\min$ & Minutes \\
\hline
\end{tabular}




\begin{tabular}{|c|c|}
\hline $\mathrm{ml}$ & Milliliters \\
\hline $\mathrm{mM}$ & Millimolar \\
\hline mRNA & Mssenger RNA \\
\hline $\mathrm{nm}$ & Nanometer \\
\hline $\mathrm{OD}_{600}$ & Absorbance at $600 \mathrm{~nm}$ \\
\hline Pmn & Puromycin \\
\hline pmol & Picomol \\
\hline poly(Pro) & poly-proline \\
\hline PRM & Parallel reaction monitoring \\
\hline P site & Peptidyl site \\
\hline RF & Release factor \\
\hline RNA & Ribonucleic acid \\
\hline rpm & Rotation per minute \\
\hline RRF & Ribosome recycling factor (RRF) \\
\hline rRNA & Ribosomal RNA \\
\hline RT & Room temperature \\
\hline$S$ & Svedberg unit \\
\hline$s$ & Seconds \\
\hline $\mathrm{T}$ & Transition state \\
\hline tRNA & Transfer RNA \\
\hline Vals & valine tRNA synthetase \\
\hline$w t$ & Wild type \\
\hline
\end{tabular}




\subsection{List of figures}

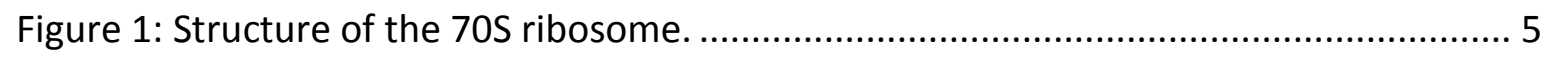

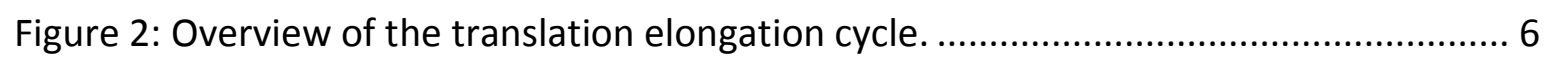

Figure 3: Comparison of peptide bond formation in solution and catalyzed by the

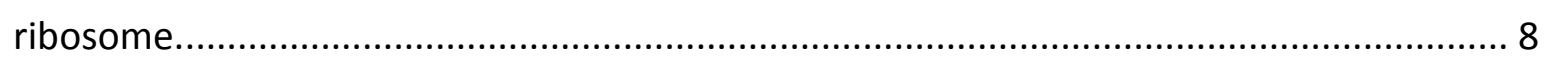

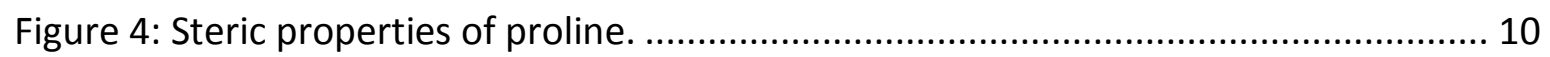

Figure 5: EF-P facilitates synthesis of poly-proline and PPG-containing peptides.............. 12

Figure 6: Structural comparison of EF-P, tRNA, elF5A and alF5A. ................................ 16

Figure 7: Post-translational modification of EF-P........................................................... 19

Figure 8: Interaction of EF-P with the mRNA, P-site tRNA and ribosomal protein L1....... 23

Figure 9: Comparison of E. coli EF-P and its homologues interacting with the P-site tRNA

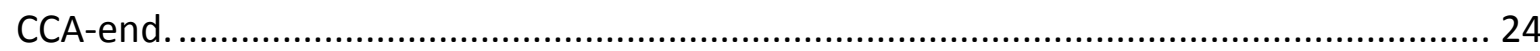

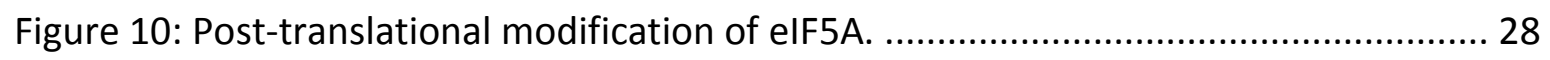

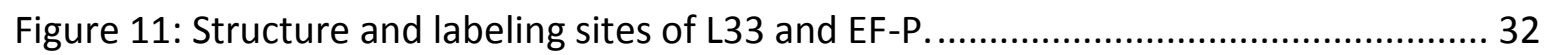

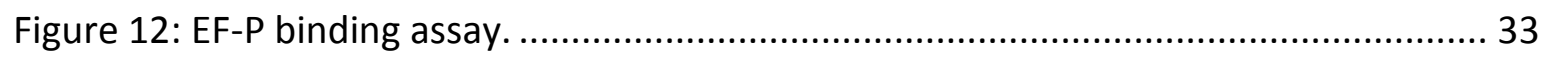

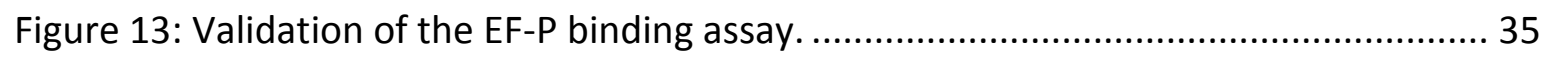

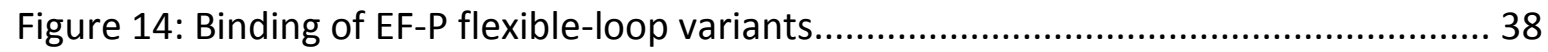

Figure 15: mRNA constructs used to study the EF-P E-site mRNA codon interaction........ 39

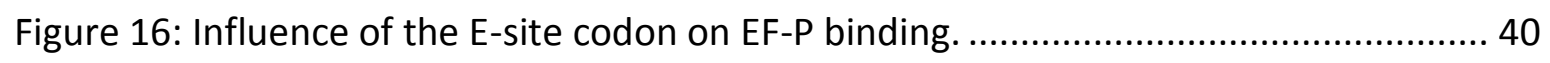

Figure 17: Binding and affinity of EF-P flexible loop variants to different E-site codons. .. 41

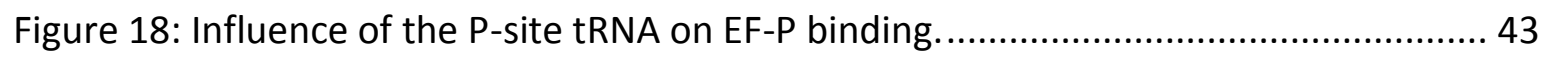

Figure 19: Effect of the $(\beta)$-hydroxy-lysinylation on EF-P binding. .................................. 46

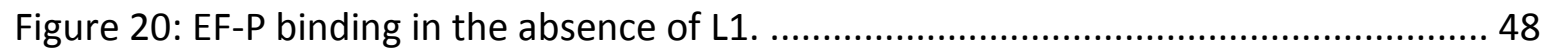

Figure 21: Influence of the E-site codon on EF-P facilitated catalysis.............................. 51

Figure 22: Effect of the modification of EF-P on peptide bond formation. ....................... 52

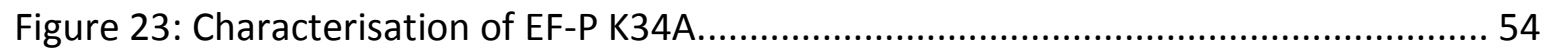

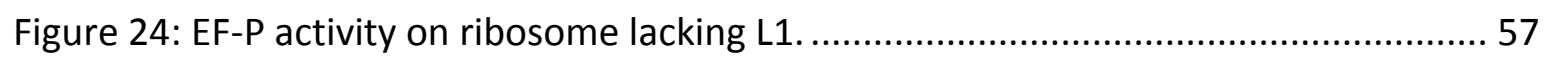

Figure 25: Binding and catalytic activity of the EFP-like protein in comparison to EF-P.... 59

Figure 26: Mass-spectrometric quantification of EF-P................................................. 62

Figure 27: Schematic depiction of the stopped-flow setup used in this study.................. 93

Figure 28: Schematic depiction of a quenched-flow apparatus. ..................................... 95

Figure 29: HPLC gradient profile of solvent B used for peptide seperation. .................... 105 
Figure 30: Thermostability of EF-P tested by ProteoPlex. 105

\subsection{List of tables}

Table 1: EF-P labeling site and distance ( $r$ ) to L33 P31C. 32

Table 2: Rates derived from EF-P interaction with ICs displaying different E-site codons. 40

Table 3: Rates of the EF-P interaction with post-translocation complexes. 44

Table 4: Rates of unmodified EF-P interacting with post-translocation complexes. 47

Table 5: Binding kinetics of ribosome binding factors 72

Table 6: List of equipment 75

Table 7: Dyes, enzymes and consumables 77

Table 8: List of columns .78

Table 9: List of software .78

Table 10: Buffers. 79

Table 11: Bacterial and eukaryotic strains 81

Table 12: EF-P labelling variants..... 83

Table 13: EF-P function variants..... 83

Table 14: EF-P fusion proteins 84

Table 15: mRNA constructs, with underlined start-codons .87

Table 16: Primers used in this study 103

\subsection{Supplementary information}

Table 16: Primers used in this study

\begin{tabular}{lll}
\hline Name & Sequence & Function \\
\hline OF3 & ggcggtGGTGGCAAACCGGCTACC & Truncation \\
OF4 & TTTGCCACCaccgccGCCCGGATCGGTATCAACG & \\
OF5 & GTTGAACTGGAAATCGTTGATgcgGATCCGGGCCTGAAAGGTG & EF-P T146A \\
OF6 & CACCTTTCAGGCCCGGATCcgcATCAACGATTTCCAGTTCAAC & \\
OF7 & GAACTGGAAATCGTTGATgtgGATCCGGGCCTGAAAGG & EF-P T146V \\
OF8 & CCTTTCAGGCCCGGATCcacATCAACGATTTCCAGTTC & \\
OF33 & Tagcaaggagatatacatctgcagccactgagatcc & EF-P YjeA YjeK
\end{tabular}


OF34.1 atgtatatctccttgctagcgTTAC

OF35 taagaaggagatatacatAcgctagcaaggagatatacatc

EF-P YjeA

OF36 atatctccttgctagcgTatgtatatctccttcttaaagttaaaca

OF37 taagaaggagatatacatggatcccctctagaaataatt EF-P

OF38.1 ATGTATATCTCCTTCTTAAAGTTAAAC

OF39* Ctgcagccactgagatcc EF-P YjeA YjeK

OF40 ggatctcagtggctgcagTTACTGCTGGCGTAGCTG

OF41 ggatctcagtggctgcagTTATGCCCGGTCAACG EF-P YjeA *

OF42 ggatctcagtggctgcagTTACTTCACGCGAGAGACG EF-P *

OF49 AAGGTatTACCGCAGGTACTGG EF-P D145L

OF50 TGCGGTAatACCTTTCAGGC

OF51 AAGGTGtgACCGCAGGTACTG EF-P D145V

OF52 TGCGGTCaCACCTTTCAGG

OF53 AAGGTGATgCgGCAGGTACTGG EF-P T146A

OF54 TACCTGCcGcATCACCTTTCAG

OF55 AAGGTGATgtgGCAGGTACTGGTG EF-P T146V

OF56 TACCTGCCacATCACCTTTCAGG

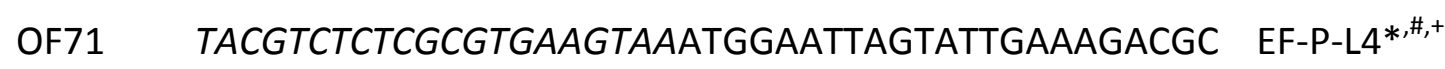

OF82 ccggatctcagtggctgcagTCATGCCAGCATCTCCTC

OF83 TACGTCTCTCGCGTGAAGTAAATGCGCCATCGTAAGAGTG EF-P-L16*,\#,+

OF74 ccggatctcagtggctgcagTTACTCTGCAGCAGCTTCTGC

OF75 TTACTTCACGCGAGAGACGTATTC

OF76 Atggctgccgcgc

OF77 tggtgccgcgcggcagccatATGTCTGACGAAGAACATACCTTTG elF5A

OF78 TTAATCGGTTCTAGCAGCTTCC

OF79 AAGCTGCTAGAACCGATTAAATGTCCCGTCCACAAGTTACTG elF5A-L4

OF84 ccggatctcagtggctgcagTTAATCGTGTTTCAAAGTTTCGG

*Using OF39, \# Using OF75,+ Using OF76 


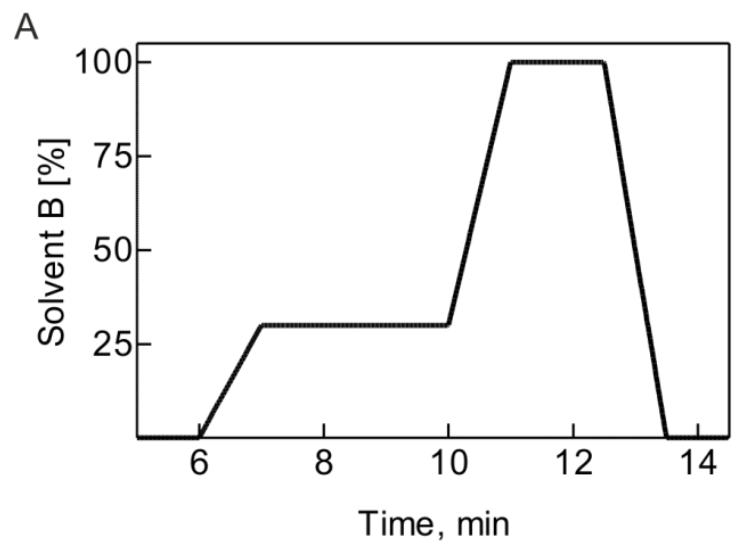

Figure 29: HPLC gradient profile of solvent B used for peptide seperation.

Apply gradient of solvent B over time during a HPLC run on a reverse-phase chromatography column to separate poly-proline containing peptides from substrates and intermediates. Using a Chromolith ${ }^{\circledR}$ Performance RP8e $100-4.6 \mathrm{~mm}$ column, solvent A $0 \%$ acetonitrile and solvent $B$ $65 \%$ acetonitrile gradient in $0.1 \%$ TFA.
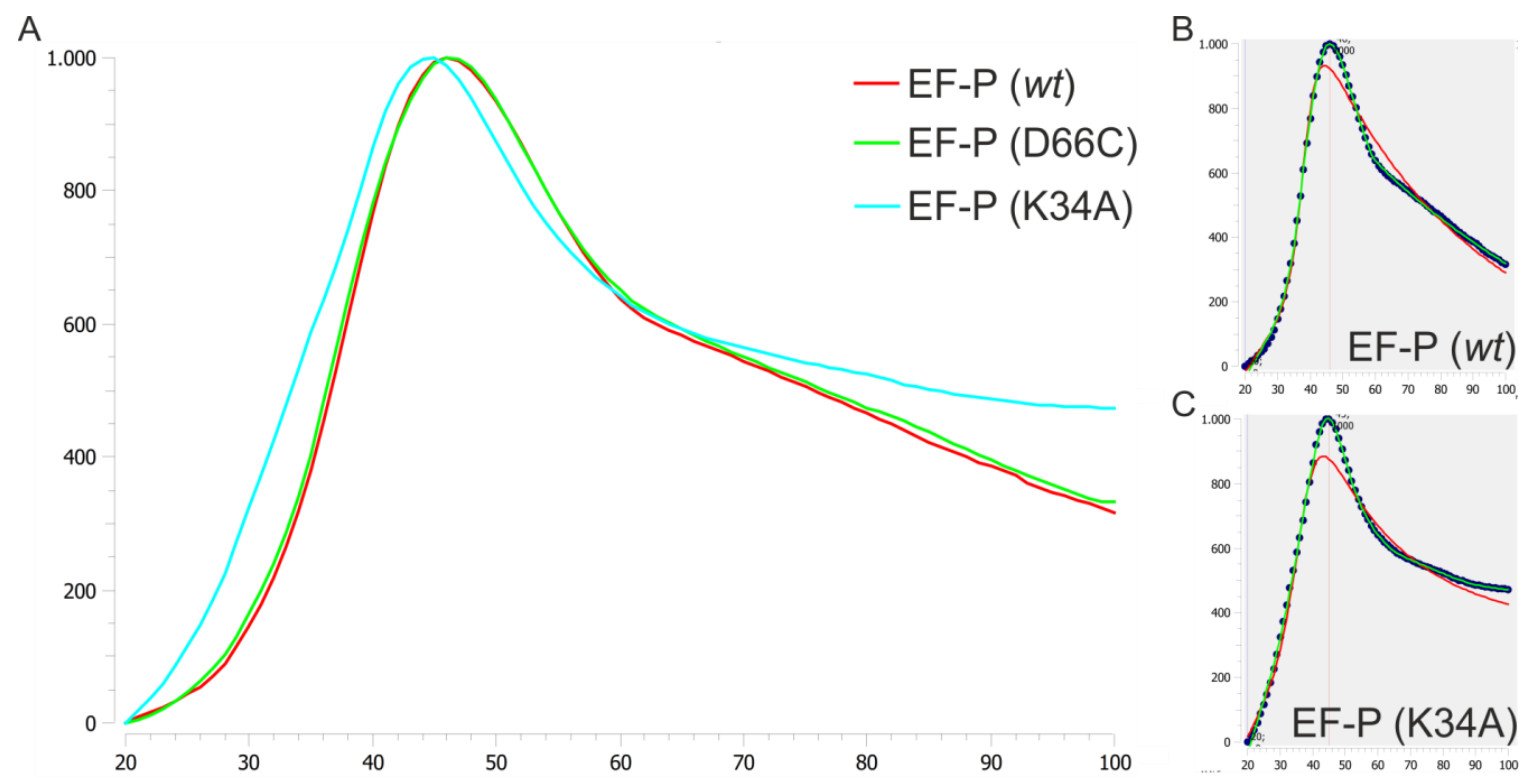

Figure 30: Thermostability of EF-P tested by ProteoPlex.

A) Comparison of the ProteoPlex analysis of EF-P (wt), EF-P (D66C) and EF-P (K34A). With increasing temperature the protein becomes unfolded, resulting in the exposure of hydrophobic side chains to the solvent. The fluorescent signal arises due to interactions of hydrophobic side chains and the dye in solution. For EF-P (wt) and the established labeling variant EF-P (D66C) the same thermostability was found, where as EF-P (K34A) appears to have a reduced thermostability. Showing the same unfolding behavior Showing the same unfolding behavior.

B) Thermostability of EF-P ( $w t)$.

C) As in B, for EF-P (K34A). 


\section{References}

Abratt, V.R., Mbewe, M., and Woods, D.R. (1998). Cloning of an EF-P homologue from Bacteroides fragilis that increases B. fragilis glutamine synthetase activity in Escherichia coli. Mol Gen Genet 258, 363-372.

Adio, S., Senyushkina, T., Peske, F., Fischer, N., Wintermeyer, W., and Rodnina, M.V. (2015). Fluctuations between multiple EF-G-induced chimeric tRNA states during translocation on the ribosome. Nat Commun 6, 7442.

Adio, S., Sharma, H., Senyushkina, T., Karki, P., Maracci, C., Wohlgemuth, I., Holtkamp, W., Peske, F., and Rodnina, M.V. (2018). Dynamics of ribosomes and release factors during translation termination in E. coli. Elife 7.

Ambrogelly, A., O'Donoghue, P., Soll, D., and Moses, S. (2010). A bacterial ortholog of class II lysyl-tRNA synthetase activates lysine. FEBS Lett 584, 3055-3060.

An, G., Glick, B.R., Friesen, J.D., and Ganoza, M.C. (1980). Identification and quantitation of elongation factor EF-P in Escherichia coli cell-free extracts. Can J Biochem 58, 13121314.

Andreotti, A.H. (2006). Opening the pore hinges on proline. Nat Chem Biol 2, 13-14.

Aoki, H., Dekany, K., Adams, S.L., and Ganoza, M.C. (1997). The gene encoding the elongation factor $\mathrm{P}$ protein is essential for viability and is required for protein synthesis. J Biol Chem 272, 32254-32259.

Aoki, H., Xu, J., Emili, A., Chosay, J.G., Golshani, A., and Ganoza, M.C. (2008). Interactions of elongation factor EF-P with the Escherichia coli ribosome. FEBS J 275, 671-681.

Baba, T., Ara, T., Hasegawa, M., Takai, Y., Okumura, Y., Baba, M., Datsenko, K.A., Tomita, M., Wanner, B.L., and Mori, H. (2006). Construction of Escherichia coli K-12 in-frame, single-gene knockout mutants: the Keio collection. Mol Syst Biol 2, 20060008.

Babaoglu, K., Page, M.A., Jones, V.C., McNeil, M.R., Dong, C., Naismith, J.H., and Lee, R.E. (2003). Novel inhibitors of an emerging target in Mycobacterium tuberculosis; substituted thiazolidinones as inhibitors of dTDP-rhamnose synthesis. Bioorg Med Chem Lett 13, 3227-3230.

Bailly, M., and de Crecy-Lagard, V. (2010). Predicting the pathway involved in posttranslational modification of elongation factor $P$ in a subset of bacterial species. Biol Direct 5, 3.

Balibar, C.J., Iwanowicz, D., and Dean, C.R. (2013). Elongation factor P is dispensable in Escherichia coli and Pseudomonas aeruginosa. Curr Microbiol 67, 293-299.

Ban, N., Nissen, P., Hansen, J., Moore, P.B., and Steitz, T.A. (2000). The complete atomic structure of the large ribosomal subunit at $2.4 \mathrm{~A}$ resolution. Science $289,905-920$.

Barbosa, N.M., Boldrin, P.E., Rossi, D., Yamamoto, P.A., Watanabe, T.F., Serrao, V.H., Hershey, J.W., Fraser, C.S., Valentini, S.R., and Zanelli, C.F. (2016). Mapping surface residues of elF5A that are important for binding to the ribosome using alanine scanning mutagenesis. Amino acids 48, 2363-2374.

Barlow, D.J., and Thornton, J.M. (1988). Helix geometry in proteins. J Mol Biol 201, 601619. 
Bartig, D., Lemkemeier, K., Frank, J., Lottspeich, F., and Klink, F. (1992). The archaebacterial hypusine-containing protein. Structural features suggest common ancestry with eukaryotic translation initiation factor 5A. Eur J Biochem 204, 751-758.

Bartig, D., Schumann, H., and Klink, F. (1990). The Unique Posttranslational Modification Leading to Deoxyhypusine or Hypusine is a General Feature of the Archaebacterial Kingdom. Systematic and Applied Microbiology 13, 112-116.

Bassani, F., Romagnoli, A., Cacciamani, T., Amici, A., Benelli, D., Londei, P., Martens, B., Blasi, U., and La Teana, A. (2018). Modification of translation factor alF5A from Sulfolobus solfataricus. Extremophiles.

Bearson, S.M., Bearson, B.L., Brunelle, B.W., Sharma, V.K., and Lee, I.S. (2011). A mutation in the poxA gene of Salmonella enterica serovar Typhimurium alters protein production, elevates susceptibility to environmental challenges, and decreases swine colonization. Foodborne Pathog Dis 8, 725-732.

Behshad, E., Ruzicka, F.J., Mansoorabadi, S.O., Chen, D., Reed, G.H., and Frey, P.A. (2006). Enantiomeric free radicals and enzymatic control of stereochemistry in a radical mechanism: the case of lysine 2,3-aminomutases. Biochemistry 45, 12639-12646.

Belardinelli, R., and Rodnina, M.V. (2017). Effect of Fusidic Acid on the Kinetics of Molecular Motions During EF-G-Induced Translocation on the Ribosome. Sci Rep 7, 10536.

Belardinelli, R., Sharma, H., Caliskan, N., Cunha, C.E., Peske, F., Wintermeyer, W., and Rodnina, M.V. (2016a). Choreography of molecular movements during ribosome progression along mRNA. Nat Struct Mol Biol 23, 342-348.

Belardinelli, R., Sharma, H., Peske, F., Wintermeyer, W., and Rodnina, M.V. (2016b). Translocation as continuous movement through the ribosome. RNA Biol 13, 1197-1203.

Benne, R., and Hershey, J.W. (1978). The mechanism of action of protein synthesis initiation factors from rabbit reticulocytes. J Biol Chem 253, 3078-3087.

Bernasconi, C.F. (1976). Relaxation kinetics, Vol 1 (Academic Press Inc.).

Bhattacharyya, R., and Chakrabarti, P. (2003). Stereospecific interactions of proline residues in protein structures and complexes. J Mol Biol 331, 925-940.

Bieling, P., Beringer, M., Adio, S., and Rodnina, M.V. (2006). Peptide bond formation does not involve acid-base catalysis by ribosomal residues. Nat Struct Mol Biol 13, 423-428.

Blaby, I.K., Phillips, G., Blaby-Haas, C.E., Gulig, K.S., El Yacoubi, B., and de Crecy-Lagard, V. (2010). Towards a systems approach in the genetic analysis of archaea: Accelerating mutant construction and phenotypic analysis in Haloferax volcanii. Archaea 2010, 426239.

Blaha, G., Stanley, R.E., and Steitz, T.A. (2009). Formation of the first peptide bond: the structure of EF-P bound to the 70S ribosome. Science 325, 966-970.

Block, K.F., Hammond, M.C., and Breaker, R.R. (2010). Evidence for widespread gene control function by the ydaO riboswitch candidate. J Bacteriol 192, 3983-3989.

Bock, L.V., Blau, C., Schroder, G.F., Davydov, II, Fischer, N., Stark, H., Rodnina, M.V., Vaiana, A.C., and Grubmuller, H. (2013). Energy barriers and driving forces in tRNA translocation through the ribosome. Nat Struct Mol Biol 20, 1390-1396. 
Bremer, H., and Dennis, P.P. (2008). Modulation of Chemical Composition and Other Parameters of the Cell at Different Exponential Growth Rates. EcoSal Plus 3.

Bullwinkle, T.J., and Ibba, M. (2016). Translation quality control is critical for bacterial responses to amino acid stress. Proc Natl Acad Sci U S A 113, 2252-2257.

Calogero, R.A., Pon, C.L., Canonaco, M.A., and Gualerzi, C.O. (1988). Selection of the mRNA translation initiation region by Escherichia coli ribosomes. Proc Natl Acad Sci U S A 85, 6427-6431.

Cano, V.S., Jeon, G.A., Johansson, H.E., Henderson, C.A., Park, J.H., Valentini, S.R., Hershey, J.W., and Park, M.H. (2008). Mutational analyses of human elF5A-1-identification of amino acid residues critical for elF5A activity and hypusine modification. FEBS J 275, 44-58.

Chakrabarti, P., and Pal, D. (2001). The interrelationships of side-chain and main-chain conformations in proteins. Prog Biophys Mol Biol 76, 1-102.

Chari, A., Haselbach, D., Kirves, J.M., Ohmer, J., Paknia, E., Fischer, N., Ganichkin, O., Moller, V., Frye, J.J., Petzold, G., et al. (2015). ProteoPlex: stability optimization of macromolecular complexes by sparse-matrix screening of chemical space. Nat Methods $12,859-865$.

Charles, T.C., and Nester, E.W. (1993). A chromosomally encoded two-component sensory transduction system is required for virulence of Agrobacterium tumefaciens. J Bacteriol $175,6614-6625$.

Chen, Y., Feng, S., Kumar, V., Ero, R., and Gao, Y.G. (2013). Structure of EF-G-ribosome complex in a pretranslocation state. Nat Struct Mol Biol 20, 1077-1084.

Choi, E., Nam, D., Choi, J., Park, S., Lee, J.S., and Lee, E.J. (2018). Elongation factor P controls translation of the mgtA gene encoding a $\mathrm{Mg}(2+)$ transporter during Salmonella infection. Microbiologyopen, e00680.

Choi, S., and Choe, J. (2011). Crystal structure of elongation factor P from Pseudomonas aeruginosa at $1.75 \mathrm{~A}$ resolution. Proteins 79, 1688-1693.

Clegg, R.M. (2002). FRET tells us about proximities, distances, orientations and dynamic properties. J Biotechnol 82, 177-179.

Cooper, H.L., Park, M.H., Folk, J.E., Safer, B., and Braverman, R. (1983). Identification of the hypusine-containing protein hy+ as translation initiation factor elF-4D. Proc Natl Acad Sci U S A 80, 1854-1857.

Cornish, P.V., Ermolenko, D.N., Noller, H.F., and Ha, T. (2008). Spontaneous intersubunit rotation in single ribosomes. Mol Cell 30, 578-588.

Cornish, P.V., Ermolenko, D.N., Staple, D.W., Hoang, L., Hickerson, R.P., Noller, H.F., and $\mathrm{Ha}, \mathrm{T}$. (2009). Following movement of the L1 stalk between three functional states in single ribosomes. Proc Natl Acad Sci U S A 106, 2571-2576.

de Crecy, E., Metzgar, D., Allen, C., Penicaud, M., Lyons, B., Hansen, C.J., and de CrecyLagard, V. (2007). Development of a novel continuous culture device for experimental evolution of bacterial populations. Appl Microbiol Biotechnol 77, 489-496.

Deana, A., Celesnik, H., and Belasco, J.G. (2008). The bacterial enzyme RppH triggers messenger RNA degradation by 5' pyrophosphate removal. Nature 451, 355-358. 
Diaconu, M., Kothe, U., Schlunzen, F., Fischer, N., Harms, J.M., Tonevitsky, A.G., Stark, H., Rodnina, M.V., and Wahl, M.C. (2005). Structural basis for the function of the ribosomal L7/12 stalk in factor binding and GTPase activation. Cell 121, 991-1004.

Dincbas-Renqvist, V., Engstrom, A., Mora, L., Heurgue-Hamard, V., Buckingham, R., and Ehrenberg, M. (2000). A post-translational modification in the GGQ motif of RF2 from Escherichia coli stimulates termination of translation. EMBO J 19, 6900-6907.

Doerfel, L.K. (2016). Function of Elongation Factor P in Translation. In Max Planck Institute for Biophysical Chemistry, Department of Physical Biochemistry (Göttingen: University of Göttingen), pp. 123.

Doerfel, L.K., Wohlgemuth, I., Kothe, C., Peske, F., Urlaub, H., and Rodnina, M.V. (2013). EF-P is essential for rapid synthesis of proteins containing consecutive proline residues. Science 339, 85-88.

Doerfel, L.K., Wohlgemuth, I., Kubyshkin, V., Starosta, A.L., Wilson, D.N., Budisa, N., and Rodnina, M.V. (2015). Entropic Contribution of Elongation Factor P to Proline Positioning at the Catalytic Center of the Ribosome. J Am Chem Soc 137, 12997-13006.

Dorner, S., Panuschka, C., Schmid, W., and Barta, A. (2003). Mononucleotide derivatives as ribosomal P-site substrates reveal an important contribution of the 2 '-OH to activity. Nucleic Acids Res 31, 6536-6542.

Elgamal, S., Artsimovitch, I., and Ibba, M. (2016). Maintenance of TranscriptionTranslation Coupling by Elongation Factor P. MBio 7.

Evans, T.C., Jr., and Nelsestuen, G.L. (1996). Importance of cis-proline 22 in the membrane-binding conformation of bovine prothrombin. Biochemistry 35, 8210-8215.

Fahnestock, S., Neumann, H., Shashoua, V., and Rich, A. (1970). Ribosome-catalyzed ester formation. Biochemistry 9, 2477-2483.

Fischer, N., Konevega, A.L., Wintermeyer, W., Rodnina, M.V., and Stark, H. (2010). Ribosome dynamics and tRNA movement by time-resolved electron cryomicroscopy. Nature 466, 329-333.

Fischer, N., Neumann, P., Konevega, A.L., Bock, L.V., Ficner, R., Rodnina, M.V., and Stark, $H$. (2015). Structure of the E. coli ribosome-EF-Tu complex at $<3$ A resolution by Cscorrected cryo-EM. Nature 520, 567-570.

Fischer, S., Dunbrack, R.L., and Karplus, M. (1994). Cis-Trans Imide Isomerization of the Proline Dipeptide. Journal of the American Chemical Society 116, 11931-11937.

Folk, J.E., Park, M.H., Chung, S.I., Schrode, J., Lester, E.P., and Cooper, H.L. (1980). Polyamines as physiological substrates for transglutaminases. J Biol Chem 255, 36953700.

Fu, J., Munro, J.B., Blanchard, S.C., and Frank, J. (2011). Cryoelectron microscopy structures of the ribosome complex in intermediate states during tRNA translocation. Proc Natl Acad Sci U S A 108, 4817-4821.

Fujino, T., Goto, Y., Suga, H., and Murakami, H. (2013). Reevaluation of the D-amino acid compatibility with the elongation event in translation. J Am Chem Soc 135, 1830-1837. 
Fukai, S., Nureki, O., Sekine, S., Shimada, A., Tao, J., Vassylyev, D.G., and Yokoyama, S. (2000). Structural basis for double-sieve discrimination of L-valine from L-isoleucine and Lthreonine by the complex of tRNA(Val) and valyl-tRNA synthetase. Cell 103, 793-803.

Fukai, S., Nureki, O., Sekine, S., Shimada, A., Vassylyev, D.G., and Yokoyama, S. (2003). Mechanism of molecular interactions for tRNA(Val) recognition by valyl-tRNA synthetase. RNA 9, 100-111.

Gamper, H.B., Masuda, I., Frenkel-Morgenstern, M., and Hou, Y.M. (2015). Maintenance of protein synthesis reading frame by EF-P and m(1)G37-tRNA. Nat Commun 6, 7226.

Ganoza, M.C., Zahid, N., and Baxter, R.M. (1985). Stimulation of peptidyltransferase reactions by a soluble protein. Eur J Biochem 146, 287-294.

Gerdes, S.Y., Scholle, M.D., Campbell, J.W., Balazsi, G., Ravasz, E., Daugherty, M.D., Somera, A.L., Kyrpides, N.C., Anderson, I., Gelfand, M.S., et al. (2003). Experimental determination and system level analysis of essential genes in Escherichia coli MG1655. J Bacteriol 185, 5673-5684.

Gibson, D.G., Young, L., Chuang, R.Y., Venter, J.C., Hutchison, C.A., 3rd, and Smith, H.O. (2009). Enzymatic assembly of DNA molecules up to several hundred kilobases. Nat Methods 6, 343-345.

Gilreath, M.S., Roy, H., Bullwinkle, T.J., Katz, A., Navarre, W.W., and Ibba, M. (2011). betaLysine discrimination by lysyl-tRNA synthetase. FEBS Lett 585, 3284-3288.

Glick, B.R., Chladek, S., and Ganoza, M.C. (1979). Peptide bond formation stimulated by protein synthesis factor EF-P depends on the aminoacyl moiety of the acceptor. Eur J Biochem 97, 23-28.

Glick, B.R., and Ganoza, M.C. (1975). Identification of a soluble protein that stimulates peptide bond synthesis. Proc Natl Acad Sci U S A 72, 4257-4260.

Goyal, A., Belardinelli, R., Maracci, C., Milon, P., and Rodnina, M.V. (2015). Directional transition from initiation to elongation in bacterial translation. Nucleic Acids Res 43, 10700-10712.

Green, R.H., Glick, B.R., and Ganoza, M.C. (1985). Requirements for in vitro reconstruction of protein synthesis. Biochem Biophys Res Commun 126, 792-798.

Gregio, A.P., Cano, V.P., Avaca, J.S., Valentini, S.R., and Zanelli, C.F. (2009). elF5A has a function in the elongation step of translation in yeast. Biochem Biophys Res Commun 380, 785-790.

Grigoriadou, C., Marzi, S., Kirillov, S., Gualerzi, C.O., and Cooperman, B.S. (2007). A quantitative kinetic scheme for $70 \mathrm{~S}$ translation initiation complex formation. J Mol Biol 373, 562-572.

Gutierrez, E., Shin, B.S., Woolstenhulme, C.J., Kim, J.R., Saini, P., Buskirk, A.R., and Dever, T.E. (2013). elF5A promotes translation of polyproline motifs. Mol Cell 51, 35-45.

Haase, N., Holtkamp, W., Lipowsky, R., Rodnina, M., and Rudorf, S. (2018). Decomposition of time-dependent fluorescence signals reveals codon-specific kinetics of protein synthesis. Nucleic Acids Res.

Hamana, K., Hayashi, H., Niitsu, M., and Itoh, T. (2009). Polyamine analysis of thermophilic, acidophilic, alkaliphilic and radio-tolerant bacteria belonging to the domain 
Bacteria and methanogens, thermophiles and extreme halophiles belonging to the domain Archaea. Journal of Japanese Society for Extremophiles 8, 59-68.

Hanawa-Suetsugu, K., Sekine, S., Sakai, H., Hori-Takemoto, C., Terada, T., Unzai, S., Tame, J.R., Kuramitsu, S., Shirouzu, M., and Yokoyama, S. (2004). Crystal structure of elongation factor P from Thermus thermophilus HB8. Proc Natl Acad Sci U S A 101, 9595-9600.

Harris, D.C. (2010). Applications of Spectrophotometry. In Quantitative Chemical Analysis (W. H. Freeman), pp. 419-444.

Helms, V. (2008). Fluorescence Resonance Energy Transfer. In Principles of Computational Cell Biology (einheim: Wiley-VCH.), p. 202.

Henderson, A., and Hershey, J.W. (2011). Eukaryotic translation initiation factor (elF) 5A stimulates protein synthesis in Saccharomyces cerevisiae. Proc Natl Acad Sci U S A 108, 6415-6419.

Hersch, S.J., Wang, M., Zou, S.B., Moon, K.M., Foster, L.J., Ibba, M., and Navarre, W.W. (2013). Divergent protein motifs direct elongation factor P-mediated translational regulation in Salmonella enterica and Escherichia coli. MBio 4, e00180-00113.

Hetrick, B., Lee, K., and Joseph, S. (2009). Kinetics of stop codon recognition by release factor 1 . Biochemistry 48, 11178-11184.

Hiller, D.A., Singh, V., Zhong, M., and Strobel, S.A. (2011). A two-step chemical mechanism for ribosome-catalysed peptide bond formation. Nature 476, 236-239.

Holtkamp, W., Wintermeyer, W., and Rodnina, M.V. (2014). Synchronous tRNA movements during translocation on the ribosome are orchestrated by elongation factor $G$ and GTP hydrolysis. Bioessays 36, 908-918.

Hovmoller, S., Zhou, T., and Ohlson, T. (2002). Conformations of amino acids in proteins. Acta Crystallogr D Biol Crystallogr 58, 768-776.

Huang, P.-Y.W., F.; Narasimhan, K.; Chatman, K.; Aach, J.; Trauger, S. A.; Spoering, R.; Church, G. M. (2017). Toward D-peptide biosynthesis: Elongation Factor P enables ribosomal incorporation of consecutive $\mathrm{D}$-amino acids bioRxiv.

Hummels, K.R., Witzky, A., Rajkovic, A., Tollerson, R., 2nd, Jones, L.A., Ibba, M., and Kearns, D.B. (2017). Carbonyl reduction by $\mathrm{Ymfl}$ in Bacillus subtilis prevents accumulation of an inhibitory EF-P modification state. Mol Microbiol 106, 236-251.

Huter, P., Arenz, S., Bock, L.V., Graf, M., Frister, J.O., Heuer, A., Peil, L., Starosta, A.L., Wohlgemuth, I., Peske, F., et al. (2017). Structural Basis for Polyproline-Mediated Ribosome Stalling and Rescue by the Translation Elongation Factor EF-P. Mol Cell 68, 515527 e516.

lannino, F., Ugalde, J.E., and Inon de lannino, N. (2012). Brucella abortus efp gene is required for an efficient internalization in HeLa cells. Microb Pathog 52, 31-40.

Ikeuchi, Y., Kimura, S., Numata, T., Nakamura, D., Yokogawa, T., Ogata, T., Wada, T., Suzuki, T., and Suzuki, T. (2010). Agmatine-conjugated cytidine in a tRNA anticodon is essential for AUA decoding in archaea. Nat Chem Biol 6, 277-282.

Ishfaq, M., Maeta, K., Maeda, S., Natsume, T., Ito, A., and Yoshida, M. (2012). Acetylation regulates subcellular localization of eukaryotic translation initiation factor $5 \mathrm{~A}$ (elF5A). FEBS Lett 586, 3236-3241. 
Ito, K., Chadani, Y., Nakamori, K., Chiba, S., Akiyama, Y., and Abo, T. (2011). Nascentome analysis uncovers futile protein synthesis in Escherichia coli. PLoS One 6, e28413.

Ito, K., Uno, M., and Nakamura, Y. (2000). A tripeptide 'anticodon' deciphers stop codons in messenger RNA. Nature 403, 680-684.

Jenko Kokalj, S., Guncar, G., Stern, I., Morgan, G., Rabzelj, S., Kenig, M., Staniforth, R.A., Waltho, J.P., Zerovnik, E., and Turk, D. (2007). Essential role of proline isomerization in stefin B tetramer formation. J Mol Biol 366, 1569-1579.

Joe, Y.A., and Park, M.H. (1994). Structural features of the elF-5A precursor required for posttranslational synthesis of deoxyhypusine. J Biol Chem 269, 25916-25921.

Joyce, A.R., Reed, J.L., White, A., Edwards, R., Osterman, A., Baba, T., Mori, H., Lesely, S.A., Palsson, B.O., and Agarwalla, S. (2006). Experimental and computational assessment of conditionally essential genes in Escherichia coli. J Bacteriol 188, 8259-8271.

Jullien, M., and Garel, J.R. (1981). Fluorescent probe of ribonuclease A conformation. Biochemistry 20, 7021-7026.

Kang, H.A., Schwelberger, H.G., and Hershey, J.W. (1993). Translation initiation factor elF$5 \mathrm{~A}$, the hypusine-containing protein, is phosphorylated on serine in Saccharomyces cerevisiae. J Biol Chem 268, 14750-14756.

Kang, K.R., Kim, Y.S., Wolff, E.C., and Park, M.H. (2007). Specificity of the deoxyhypusine hydroxylase-eukaryotic translation initiation factor (elF5A) interaction: identification of amino acid residues of the enzyme required for binding of its substrate, deoxyhypusinecontaining elF5A. J Biol Chem 282, 8300-8308.

Kaniga, K., Compton, M.S., Curtiss, R., 3rd, and Sundaram, P. (1998). Molecular and functional characterization of Salmonella enterica serovar typhimurium poxA gene: effect on attenuation of virulence and protection. Infect Immun 66, 5599-5606.

Katoh, T., Iwane, Y., and Suga, H. (2017a). Logical engineering of D-arm and T-stem of tRNA that enhances d-amino acid incorporation. Nucleic Acids Res 45, 12601-12610.

Katoh, T., and Suga, H. (2018). Ribosomal Incorporation of Consecutive beta-Amino Acids. J Am Chem Soc.

Katoh, T., Tajima, K., and Suga, H. (2017b). Consecutive Elongation of D-Amino Acids in Translation. Cell Chem Biol 24, 46-54.

Katoh, T., Wohlgemuth, I., Nagano, M., Rodnina, M.V., and Suga, H. (2016). Essential structural elements in tRNA(Pro) for EF-P-mediated alleviation of translation stalling. Nat Commun 7, 11657.

Katunin, V.I., Muth, G.W., Strobel, S.A., Wintermeyer, W., and Rodnina, M.V. (2002). Important contribution to catalysis of peptide bond formation by a single ionizing group within the ribosome. Mol Cell 10, 339-346.

Katz, A., Solden, L., Zou, S.B., Navarre, W.W., and Ibba, M. (2014). Molecular evolution of protein-RNA mimicry as a mechanism for translational control. Nucleic Acids Res 42, 3261-3271.

Kawakami, T., and Murakami, H. (2012). Genetically encoded libraries of nonstandard peptides. J Nucleic Acids 2012, 713510. 
Kazemi, M., Socan, J., Himo, F., and Aqvist, J. (2018). Mechanistic alternatives for peptide bond formation on the ribosome. Nucleic Acids Res 46, 5345-5354.

Kim, K.K., Hung, L.W., Yokota, H., Kim, R., and Kim, S.H. (1998). Crystal structures of eukaryotic translation initiation factor $5 \mathrm{~A}$ from Methanococcus jannaschii at $1.8 \mathrm{~A}$ resolution. Proc Natl Acad Sci U S A 95, 10419-10424.

Klee, S.M., Mostafa, I., Chen, S., Dufresne, C., Lehman, B.L., Sinn, J.P., Peter, K.A., and McNellis, T.W. (2018). An Erwinia amylovora yjeK mutant exhibits reduced virulence, increased chemical sensitivity and numerous environmentally dependent proteomic alterations. Mol Plant Pathol 19, 1667-1678.

Klier, H., Wohl, T., Eckerskorn, C., Magdolen, V., and Lottspeich, F. (1993). Determination and mutational analysis of the phosphorylation site in the hypusine-containing protein Hyp2p. FEBS Lett 334, 360-364.

Klostermeier, D.R., M.G. (2017). Biophysical Chemistry, Vol 1 (CRC Press ).

Kobayashi, K., Katz, A., Rajkovic, A., Ishii, R., Branson, O.E., Freitas, M.A., Ishitani, R., Ibba, M., and Nureki, O. (2014a). The non-canonical hydroxylase structure of YfcM reveals a metal ion-coordination motif required for EF-P hydroxylation. Nucleic Acids Res 42, 12295-12305.

Kobayashi, K., Suzuki, T., Dohmae, N., Ishitani, R., and Nureki, O. (2014b). Crystallization and preliminary X-ray crystallographic analysis of $\mathrm{YfCM}$ : an important factor for EF-P hydroxylation. Acta Crystallogr F Struct Biol Commun 70, 1236-1239.

Kothe, U., and Rodnina, M.V. (2006). Delayed release of inorganic phosphate from elongation factor Tu following GTP hydrolysis on the ribosome. Biochemistry 45, 1276712774.

Kothe, U., Wieden, H.J., Mohr, D., and Rodnina, M.V. (2004). Interaction of helix D of elongation factor Tu with helices 4 and 5 of protein L7/12 on the ribosome. J Mol Biol 336, 1011-1021.

Krafczyk, R., Macosek, J., Jagtap, P.K.A., Gast, D., Wunder, S., Mitra, P., Jha, A.K., Rohr, J., Hoffmann-Roder, A., Jung, K., et al. (2017). Structural Basis for EarP-Mediated Arginine Glycosylation of Translation Elongation Factor EF-P. MBio 8.

Kuhlenkoetter, S., Wintermeyer, W., and Rodnina, M.V. (2011). Different substratedependent transition states in the active site of the ribosome. Nature 476, 351-354.

Kulak, N.A., Pichler, G., Paron, I., Nagaraj, N., and Mann, M. (2014). Minimal, encapsulated proteomic-sample processing applied to copy-number estimation in eukaryotic cells. Nat Methods 11, 319-324.

Laemmli, U.K. (1970). Cleavage of structural proteins during the assembly of the head of bacteriophage T4. Nature 227, 680-685.

Lam, J.S., Taylor, V.L., Islam, S.T., Hao, Y., and Kocincova, D. (2011). Genetic and Functional Diversity of Pseudomonas aeruginosa Lipopolysaccharide. Front Microbiol 2, 118.

Langridge, G.C., Phan, M.D., Turner, D.J., Perkins, T.T., Parts, L., Haase, J., Charles, I., Maskell, D.J., Peters, S.E., Dougan, G., et al. (2009). Simultaneous assay of every Salmonella Typhi gene using one million transposon mutants. Genome Res 19, 2308-2316. 
Lassak, J., Keilhauer, E.C., Furst, M., Wuichet, K., Godeke, J., Starosta, A.L., Chen, J.M., Sogaard-Andersen, L., Rohr, J., Wilson, D.N., et al. (2015). Arginine-rhamnosylation as new strategy to activate translation elongation factor P. Nat Chem Biol 11, 266-270.

Lassak, J., Wilson, D.N., and Jung, K. (2016). Stall no more at polyproline stretches with the translation elongation factors EF-P and IF-5A. Mol Microbiol 99, 219-235.

Ledoux, S., and Uhlenbeck, O.C. (2008). Different aa-tRNAs are selected uniformly on the ribosome. Mol Cell 31, 114-123.

Lee, C.H., and Park, M.H. (2000). Human deoxyhypusine synthase: interrelationship between binding of NAD and substrates. Biochem J 352 Pt 3, 851-857.

Li, S.C., Goto, N.K., Williams, K.A., and Deber, C.M. (1996). Alpha-helical, but not betasheet, propensity of proline is determined by peptide environment. Proc Natl Acad Sci U S A 93, 6676-6681.

Li, X., Krafczyk, R., Macosek, J., Li, Y.L., Zou, Y., Simon, B., Pan, X., Wu, Q.Y., Yan, F., Li, S., et al. (2016). Resolving the alpha-glycosidic linkage of arginine-rhamnosylated translation elongation factor $P$ triggers generation of the first $\operatorname{Arg}($ Rha) specific antibody. Chem Sci 7 , 6995-7001.

Lieberman, K.R., and Dahlberg, A.E. (1995). Ribosome-catalyzed peptide-bond formation. Prog Nucleic Acid Res Mol Biol 50, 1-23.

Lindhout, T., Lau, P.C., Brewer, D., and Lam, J.S. (2009). Truncation in the core oligosaccharide of lipopolysaccharide affects flagella-mediated motility in Pseudomonas aeruginosa PAO1 via modulation of cell surface attachment. Microbiology 155, 34493460.

Liu, C.C., and Schultz, P.G. (2010). Adding new chemistries to the genetic code. Annu Rev Biochem 79, 413-444.

Lummis, S.C., Beene, D.L., Lee, L.W., Lester, H.A., Broadhurst, R.W., and Dougherty, D.A. (2005). Cis-trans isomerization at a proline opens the pore of a neurotransmitter-gated ion channel. Nature 438, 248-252.

Maass, S., Sievers, S., Zuhlke, D., Kuzinski, J., Sappa, P.K., Muntel, J., Hessling, B., Bernhardt, J., Sietmann, R., Volker, U., et al. (2011). Efficient, global-scale quantification of absolute protein amounts by integration of targeted mass spectrometry and twodimensional gel-based proteomics. Anal Chem 83, 2677-2684.

MacArthur, M.W., and Thornton, J.M. (1991). Influence of proline residues on protein conformation. J Mol Biol 218, 397-412.

Maloy, S.R., Stewart, V.J., and Taylor, R.K. (1996). Genetic analysis of pathogenic bacteria: a laboratory manual ( Cold Spring Harbor Laboratory Press).

Mandal, D., Kohrer, C., Su, D., Russell, S.P., Krivos, K., Castleberry, C.M., Blum, P., Limbach, P.A., Soll, D., and RajBhandary, U.L. (2010). Agmatidine, a modified cytidine in the anticodon of archaeal tRNA(Ile), base pairs with adenosine but not with guanosine. Proc Natl Acad Sci U S A 107, 2872-2877.

Marman, H.E., Mey, A.R., and Payne, S.M. (2014). Elongation factor P and modifying enzyme PoxA are necessary for virulence of Shigella flexneri. Infect Immun 82, 3612-3621. 
Matheisl, S., Berninghausen, O., Becker, T., and Beckmann, R. (2015). Structure of a human translation termination complex. Nucleic Acids Res 43, 8615-8626.

Meeske, A.J., Rodrigues, C.D., Brady, J., Lim, H.C., Bernhardt, T.G., and Rudner, D.Z. (2016). High-Throughput Genetic Screens Identify a Large and Diverse Collection of New Sporulation Genes in Bacillus subtilis. PLoS Biol 14, e1002341.

Melnikov, S., Mailliot, J., Shin, B.S., Rigger, L., Yusupova, G., Micura, R., Dever, T.E., and Yusupov, M. (2016). Crystal Structure of Hypusine-Containing Translation Factor elF5A Bound to a Rotated Eukaryotic Ribosome. J Mol Biol 428, 3570-3576.

Mercier, E., and Rodnina, M.V. (2018). Co-Translational Folding Trajectory of the HemK Helical Domain. Biochemistry 57, 3460-3464.

Michaelis, L.S., M. P. (1934). The reaction of iodoacetic acid on mercaptans and amines. J Biol Chem 106, 331-341.

Milon, P., Konevega, A.L., Gualerzi, C.O., and Rodnina, M.V. (2008). Kinetic checkpoint at a late step in translation initiation. Mol Cell 30, 712-720.

Milon, P., Maracci, C., Filonava, L., Gualerzi, C.O., and Rodnina, M.V. (2012). Real-time assembly landscape of bacterial $30 \mathrm{~S}$ translation initiation complex. Nat Struct Mol Biol 19, 609-615.

Milon, P., and Rodnina, M.V. (2012). Kinetic control of translation initiation in bacteria. Crit Rev Biochem Mol Biol 47, 334-348.

Mittelstaet, J., Konevega, A.L., and Rodnina, M.V. (2011). Distortion of tRNA upon nearcognate codon recognition on the ribosome. J Biol Chem 286, 8158-8164.

Moazed, D., and Noller, H.F. (1989). Intermediate states in the movement of transfer RNA in the ribosome. Nature 342, 142-148.

Mohapatra, S., Choi, H., Ge, X., Sanyal, S., and Weisshaar, J.C. (2017). Spatial Distribution and Ribosome-Binding Dynamics of EF-P in Live Escherichia coli. MBio 8.

Moine, H., Romby, P., Springer, M., Grunberg-Manago, M., Ebel, J.P., Ehresmann, C., and Ehresmann, B. (1988). Messenger RNA structure and gene regulation at the translational level in Escherichia coli: the case of threonine:tRNAThr ligase. Proc Natl Acad Sci U S A 85, 7892-7896.

Munro, J.B., Altman, R.B., Tung, C.S., Cate, J.H., Sanbonmatsu, K.Y., and Blanchard, S.C. (2010). Spontaneous formation of the unlocked state of the ribosome is a multistep process. Proc Natl Acad Sci U S A 107, 709-714.

Murphey, R.J., and Gerner, E.W. (1987). Hypusine formation in protein by a two-step process in cell lysates. J Biol Chem 262, 15033-15036.

Muto, H., and Ito, K. (2008). Peptidyl-prolyl-tRNA at the ribosomal P-site reacts poorly with puromycin. Biochem Biophys Res Commun 366, 1043-1047.

Nakahigashi, K., Kubo, N., Narita, S., Shimaoka, T., Goto, S., Oshima, T., Mori, H., Maeda, M., Wada, C., and Inokuchi, H. (2002). HemK, a class of protein methyl transferase with similarity to DNA methyl transferases, methylates polypeptide chain release factors, and hemK knockout induces defects in translational termination. Proc Natl Acad Sci U S A 99, 1473-1478. 
Navarre, W.W., Zou, S.B., Roy, H., Xie, J.L., Savchenko, A., Singer, A., Edvokimova, E., Prost, L.R., Kumar, R., Ibba, M., et al. (2010). PoxA, yjeK, and elongation factor $P$ coordinately modulate virulence and drug resistance in Salmonella enterica. Mol Cell 39, 209-221.

Nissen, P., Hansen, J., Ban, N., Moore, P.B., and Steitz, T.A. (2000). The structural basis of ribosome activity in peptide bond synthesis. Science 289, 920-930.

Noller, H.F., Hoffarth, V., and Zimniak, L. (1992). Unusual resistance of peptidyl transferase to protein extraction procedures. Science 256, 1416-1419.

Ogle, J.M., Brodersen, D.E., Clemons, W.M., Jr., Tarry, M.J., Carter, A.P., and Ramakrishnan, V. (2001). Recognition of cognate transfer RNA by the 30 s ribosomal subunit. Science 292, 897-902.

Ogle, J.M., Murphy, F.V., Tarry, M.J., and Ramakrishnan, V. (2002). Selection of tRNA by the ribosome requires a transition from an open to a closed form. Cell 111, 721-732.

Ohashi, Y., Inaoka, T., Kasai, K., Ito, Y., Okamoto, S., Satsu, H., Tozawa, Y., Kawamura, F., and Ochi, K. (2003). Expression profiling of translation-associated genes in sporulating Bacillus subtilis and consequence of sporulation by gene inactivation. Biosci Biotechnol Biochem 67, 2245-2253.

Owens, N.W., Braun, C., O'Neil, J.D., Marat, K., and Schweizer, F. (2007). Effects of glycosylation of $(2 \mathrm{~S}, 4 \mathrm{R})$-4-hydroxyproline on the conformation, kinetics, and thermodynamics of prolyl amide isomerization. J Am Chem Soc 129, 11670-11671.

Park, J.H., Aravind, L., Wolff, E.C., Kaevel, J., Kim, Y.S., and Park, M.H. (2006). Molecular cloning, expression, and structural prediction of deoxyhypusine hydroxylase: a HEATrepeat-containing metalloenzyme. Proc Natl Acad Sci U S A 103, 51-56.

Park, J.H., Dias, C.A., Lee, S.B., Valentini, S.R., Sokabe, M., Fraser, C.S., and Park, M.H. (2011). Production of active recombinant elF5A: reconstitution in E.coli of eukaryotic hypusine modification of elF5A by its coexpression with modifying enzymes. Protein Eng Des Sel 24, 301-309.

Park, J.H., Wolff, E.C., Folk, J.E., and Park, M.H. (2003). Reversal of the deoxyhypusine synthesis reaction. Generation of spermidine or homospermidine from deoxyhypusine by deoxyhypusine synthase. J Biol Chem 278, 32683-32691.

Park, M.H. (1987). Regulation of biosynthesis of hypusine in Chinese hamster ovary cells. Evidence for elF-4D precursor polypeptides. J Biol Chem 262, 12730-12734.

Park, M.H. (1989). The essential role of hypusine in eukaryotic translation initiation factor 4D (elF-4D). Purification of elF-4D and its precursors and comparison of their activities. J Biol Chem 264, 18531-18535.

Park, M.H. (2006). The post-translational synthesis of a polyamine-derived amino acid, hypusine, in the eukaryotic translation initiation factor 5A (elF5A). J Biochem 139, 161169.

Park, M.H., Cooper, H.L., and Folk, J.E. (1981). Identification of hypusine, an unusual amino acid, in a protein from human lymphocytes and of spermidine as its biosynthetic precursor. Proc Natl Acad Sci U S A 78, 2869-2873. 
Park, M.H., Cooper, H.L., and Folk, J.E. (1982). The biosynthesis of protein-bound hypusine ( $\mathrm{N}$ epsilon -(4-amino-2-hydroxybutyl)lysine). Lysine as the amino acid precursor and the intermediate role of deoxyhypusine (N epsilon -(4-aminobutyl)lysine). J Biol Chem 257, 7217-7222.

Park, M.H., Joe, Y.A., and Kang, K.R. (1998). Deoxyhypusine synthase activity is essential for cell viability in the yeast Saccharomyces cerevisiae. J Biol Chem 273, 1677-1683.

Park, M.H., Nishimura, K., Zanelli, C.F., and Valentini, S.R. (2010). Functional significance of elF5A and its hypusine modification in eukaryotes. Amino acids 38, 491-500.

Park, M.H., Wolff, E.C., Smit-McBride, Z., Hershey, J.W., and Folk, J.E. (1991). Comparison of the activities of variant forms of elF-4D. The requirement for hypusine or deoxyhypusine. J Biol Chem 266, 7988-7994.

Patel, P.H., Costa-Mattioli, M., Schulze, K.L., and Bellen, H.J. (2009). The Drosophila deoxyhypusine hydroxylase homologue nero and its target elF5A are required for cell growth and the regulation of autophagy. J Cell Biol 185, 1181-1194.

Pavlov, M.Y., Watts, R.E., Tan, Z., Cornish, V.W., Ehrenberg, M., and Forster, A.C. (2009). Slow peptide bond formation by proline and other $\mathrm{N}$-alkylamino acids in translation. Proc Natl Acad Sci U S A 106, 50-54.

Peil, L., Starosta, A.L., Lassak, J., Atkinson, G.C., Virumae, K., Spitzer, M., Tenson, T., Jung, K., Remme, J., and Wilson, D.N. (2013). Distinct XPPX sequence motifs induce ribosome stalling, which is rescued by the translation elongation factor EF-P. Proc Natl Acad Sci U S A 110, 15265-15270.

Peil, L., Starosta, A.L., Virumae, K., Atkinson, G.C., Tenson, T., Remme, J., and Wilson, D.N. (2012). Lys34 of translation elongation factor EF-P is hydroxylated by $\mathrm{YfcM}$. Nat Chem Biol 8, 695-697.

Pelechano, V., and Alepuz, P. (2017). elF5A facilitates translation termination globally and promotes the elongation of many non polyproline-specific tripeptide sequences. Nucleic Acids Res 45, 7326-7338.

Peng, W.T., Banta, L.M., Charles, T.C., and Nester, E.W. (2001). The chvH locus of Agrobacterium encodes a homologue of an elongation factor involved in protein synthesis. J Bacteriol 183, 36-45.

Peske, F., Kuhlenkoetter, S., Rodnina, M.V., and Wintermeyer, W. (2014). Timing of GTP binding and hydrolysis by translation termination factor RF3. Nucleic Acids Res 42, 18121820.

Peske, F., Rodnina, M.V., and Wintermeyer, W. (2005). Sequence of steps in ribosome recycling as defined by kinetic analysis. Mol Cell 18, 403-412.

Pierson, W.E., Hoffer, E.D., Keedy, H.E., Simms, C.L., Dunham, C.M., and Zaher, H.S. (2016). Uniformity of Peptide Release Is Maintained by Methylation of Release Factors. Cell Rep 17, 11-18.

Polikanov, Y.S., Steitz, T.A., and Innis, C.A. (2014). A proton wire to couple aminoacyltRNA accommodation and peptide-bond formation on the ribosome. Nat Struct Mol Biol 21, 787-793. 
Prunetti, L., Graf, M., Blaby, I.K., Peil, L., Makkay, A.M., Starosta, A.L., Papke, R.T., Oshima, T., Wilson, D.N., and de Crecy-Lagard, V. (2016). Deciphering the Translation Initiation Factor 5A Modification Pathway in Halophilic Archaea. Archaea 2016, 7316725.

Quintas-Granados, L.I., Carvajal Gamez, B.I., Villalpando, J.L., Ortega-Lopez, J., Arroyo, R., Azuara-Liceaga, E., and Alvarez-Sanchez, M.E. (2016). Bifunctional activity of deoxyhypusine synthase/hydroxylase from Trichomonas vaginalis. Biochimie 123, 37-51.

Rahim, R., Ochsner, U.A., Olvera, C., Graninger, M., Messner, P., Lam, J.S., and SoberonChavez, G. (2001). Cloning and functional characterization of the Pseudomonas aeruginosa rhlC gene that encodes rhamnosyltransferase 2 , an enzyme responsible for dirhamnolipid biosynthesis. Mol Microbiol 40, 708-718.

Rajkovic, A., Hummels, K.R., Witzky, A., Erickson, S., Gafken, P.R., Whitelegge, J.P., Faull, K.F., Kearns, D.B., and Ibba, M. (2016). Translation Control of Swarming Proficiency in Bacillus subtilis by 5-Amino-pentanolylated Elongation Factor P. J Biol Chem 291, 1097610985.

Rajkovic, A., and Ibba, M. (2017). Elongation Factor $P$ and the Control of Translation Elongation. Annu Rev Microbiol 71, 117-131.

Ramachandran, G.N., Lakshminarayanan, A.V., Balasubramanian, R., and Tegoni, G. (1970). Studies on the conformation of amino acids. XII. Energy calculations on prolyl residue. Biochim Biophys Acta 221, 165-181.

Ranjan, N., and Rodnina, M.V. (2017). Thio-Modification of tRNA at the Wobble Position as Regulator of the Kinetics of Decoding and Translocation on the Ribosome. J Am Chem Soc $139,5857-5864$.

Richards, J., Luciano, D.J., and Belasco, J.G. (2012). Influence of translation on RppHdependent mRNA degradation in Escherichia coli. Mol Microbiol 86, 1063-1072.

Rivas, G., and Minton, A.P. (2016). Macromolecular Crowding In Vitro, In Vivo, and In Between. Trends Biochem Sci 41, 970-981.

Rodnina, M.V. (2013). The ribosome as a versatile catalyst: reactions at the peptidyl transferase center. Curr Opin Struct Biol 23, 595-602.

Rodnina, M.V. (2016). The ribosome in action: Tuning of translational efficiency and protein folding. Protein Sci 25, 1390-1406.

Rodnina, M.V., Savelsbergh, A., Katunin, V.I., and Wintermeyer, W. (1997). Hydrolysis of GTP by elongation factor $G$ drives tRNA movement on the ribosome. Nature 385, 37-41.

Rodnina, M.V., and Wintermeyer, W. (1995). GTP consumption of elongation factor Tu during translation of heteropolymeric mRNAs. Proc Natl Acad Sci U S A 92, 1945-1949.

Rodnina, M.V., and Wintermeyer, W. (2011). The ribosome as a molecular machine: the mechanism of tRNA-mRNA movement in translocation. Biochem Soc Trans 39, 658-662.

Rodnina, M.V., and Wintermeyer, W. (2016). Protein Elongation, Co-translational Folding and Targeting. J Mol Biol 428, 2165-2185.

Rossi, D., Barbosa, N.M., Galvao, F.C., Boldrin, P.E., Hershey, J.W., Zanelli, C.F., Fraser, C.S., and Valentini, S.R. (2016). Evidence for a Negative Cooperativity between elF5A and eEF2 on Binding to the Ribosome. PLoS One 11, e0154205. 
Roy, H., Zou, S.B., Bullwinkle, T.J., Wolfe, B.S., Gilreath, M.S., Forsyth, C.J., Navarre, W.W., and Ibba, M. (2011). The tRNA synthetase paralog PoxA modifies elongation factor-P with (R)-beta-lysine. Nat Chem Biol 7, 667-669.

Rudorf, S., Thommen, M., Rodnina, M.V., and Lipowsky, R. (2014). Deducing the kinetics of protein synthesis in vivo from the transition rates measured in vitro. PLoS Comput Biol 10, e1003909.

Saini, P., Eyler, D.E., Green, R., and Dever, T.E. (2009). Hypusine-containing protein elF5A promotes translation elongation. Nature 459, 118-121.

Saks, M.E., Sampson, J.R., and Abelson, J.N. (1994). The transfer RNA identity problem: a search for rules. Science 263, 191-197.

Sampson, J.R., and Uhlenbeck, O.C. (1988). Biochemical and physical characterization of an unmodified yeast phenylalanine transfer RNA transcribed in vitro. Proc Natl Acad Sci U S A 85, 1033-1037.

Sarkar, P., Reichman, C., Saleh, T., Birge, R.B., and Kalodimos, C.G. (2007). Proline cis-trans isomerization controls autoinhibition of a signaling protein. Mol Cell 25, 413-426.

Sasaki, K., Abid, M.R., and Miyazaki, M. (1996). Deoxyhypusine synthase gene is essential for cell viability in the yeast Saccharomyces cerevisiae. FEBS Lett 384, 151-154.

Satterthwait, A.C., and Jencks, W.P. (1974). The mechanism of the aminolysis of acetate esters. J Am Chem Soc 96, 7018-7031.

Savelsbergh, A., Katunin, V.I., Mohr, D., Peske, F., Rodnina, M.V., and Wintermeyer, W. (2003). An elongation factor G-induced ribosome rearrangement precedes tRNA-mRNA translocation. Mol Cell 11, 1517-1523.

Schavemaker, P.E., Smigiel, W.M., and Poolman, B. (2017). Ribosome surface properties may impose limits on the nature of the cytoplasmic proteome. Elife 6.

Schirm, M., Arora, S.K., Verma, A., Vinogradov, E., Thibault, P., Ramphal, R., and Logan, S.M. (2004). Structural and genetic characterization of glycosylation of type a flagellin in Pseudomonas aeruginosa. J Bacteriol 186, 2523-2531.

Schmeing, T.M., Huang, K.S., Kitchen, D.E., Strobel, S.A., and Steitz, T.A. (2005). Structural insights into the roles of water and the 2 ' hydroxyl of the P site tRNA in the peptidyl transferase reaction. Mol Cell 20, 437-448.

Schmeing, T.M., Voorhees, R.M., Kelley, A.C., Gao, Y.G., Murphy, F.V.t., Weir, J.R., and Ramakrishnan, V. (2009). The crystal structure of the ribosome bound to EF-Tu and aminoacyl-tRNA. Science 326, 688-694.

Schmidt, A., Kochanowski, K., Vedelaar, S., Ahrne, E., Volkmer, B., Callipo, L., Knoops, K., Bauer, M., Aebersold, R., and Heinemann, M. (2016a). The quantitative and conditiondependent Escherichia coli proteome. Nat Biotechnol 34, 104-110.

Schmidt, C., Becker, T., Heuer, A., Braunger, K., Shanmuganathan, V., Pech, M., Berninghausen, O., Wilson, D.N., and Beckmann, R. (2016b). Structure of the hypusinylated eukaryotic translation factor elF-5A bound to the ribosome. Nucleic Acids Res 44, 1944-1951. 
Schreier, M.H., Erni, B., and Staehelin, T. (1977). Initiation of mammalian protein synthesis. I. Purification and characterization of seven initiation factors. J Mol Biol 116, 727-753.

Schuller, A.P., Wu, C.C., Dever, T.E., Buskirk, A.R., and Green, R. (2017). elF5A Functions Globally in Translation Elongation and Termination. Mol Cell 66, 194-205 e195.

Selmer, M., Dunham, C.M., Murphy, F.V.t., Weixlbaumer, A., Petry, S., Kelley, A.C., Weir, J.R., and Ramakrishnan, V. (2006). Structure of the 70S ribosome complexed with mRNA and tRNA. Science 313, 1935-1942.

Sengoku, T., Suzuki, T., Dohmae, N., Watanabe, C., Honma, T., Hikida, Y., Yamaguchi, Y., Takahashi, H., Yokoyama, S., and Yanagisawa, T. (2018). Structural basis of protein arginine rhamnosylation by glycosyltransferase EarP. Nat Chem Biol 14, 368-374.

Shampo, M.A., and Kyle, R.A. (2002). Kary B. Mullis--Nobel Laureate for procedure to replicate DNA. Mayo Clin Proc 77, 606.

Sharma, H., Adio, S., Senyushkina, T., Belardinelli, R., Peske, F., and Rodnina, M.V. (2016). Kinetics of Spontaneous and EF-G-Accelerated Rotation of Ribosomal Subunits. Cell Rep 16, 2187-2196.

Shaw, J.J., and Green, R. (2007). Two distinct components of release factor function uncovered by nucleophile partitioning analysis. Mol Cell 28, 458-467.

Shine, J., and Dalgarno, L. (1974). The 3'-terminal sequence of Escherichia coli $16 \mathrm{~S}$ ribosomal RNA: complementarity to nonsense triplets and ribosome binding sites. Proc Natl Acad Sci U S A 71, 1342-1346.

Sievert, H., Pallmann, N., Miller, K.K., Hermans-Borgmeyer, I., Venz, S., Sendoel, A., Preukschas, M., Schweizer, M., Boettcher, S., Janiesch, P.C., et al. (2014). A novel mouse model for inhibition of $\mathrm{DOHH}$-mediated hypusine modification reveals a crucial function in embryonic development, proliferation and oncogenic transformation. Dis Model Mech 7, 963-976.

Sohmen, D., Chiba, S., Shimokawa-Chiba, N., Innis, C.A., Berninghausen, O., Beckmann, R., Ito, K., and Wilson, D.N. (2015). Structure of the Bacillus subtilis $70 S$ ribosome reveals the basis for species-specific stalling. Nat Commun 6, 6941.

Solbak, S.M., Reksten, T.R., Wray, V., Bruns, K., Horvli, O., Raae, A.J., Henklein, P., Henklein, P., Roder, R., Mitzner, D., et al. (2010). The intriguing cyclophilin A-HIV-1 Vpr interaction: prolyl cis/trans isomerisation catalysis and specific binding. BMC Struct Biol $10,31$.

Starosta, A.L., Lassak, J., Peil, L., Atkinson, G.C., Virumae, K., Tenson, T., Remme, J., Jung, K., and Wilson, D.N. (2014a). Translational stalling at polyproline stretches is modulated by the sequence context upstream of the stall site. Nucleic Acids Res 42, 10711-10719.

Starosta, A.L., Lassak, J., Peil, L., Atkinson, G.C., Woolstenhulme, C.J., Virumae, K., Buskirk, A., Tenson, T., Remme, J., Jung, K., et al. (2014b). A conserved proline triplet in Val-tRNA synthetase and the origin of elongation factor P. Cell Rep 9, 476-483.

Studer, S.M., and Joseph, S. (2006). Unfolding of mRNA secondary structure by the bacterial translation initiation complex. Mol Cell 22, 105-115. 
Su, T., Cheng, J., Sohmen, D., Hedman, R., Berninghausen, O., von Heijne, G., Wilson, D.N., and Beckmann, R. (2017). The force-sensing peptide VemP employs extreme compaction and secondary structure formation to induce ribosomal stalling. Elife 6 .

Sumida, T., Yanagisawa, T., Ishii, R., and Yokoyama, S. (2010). Crystallization and preliminary X-ray crystallographic study of GenX, a lysyl-tRNA synthetase paralogue from Escherichia coli, in complex with translation elongation factor P. Acta Crystallogr Sect $F$ Struct Biol Cryst Commun 66, 1115-1118.

Thompson, M.W., Govindaswami, M., and Hersh, L.B. (2003). Mutation of active site residues of the puromycin-sensitive aminopeptidase: conversion of the enzyme into a catalytically inactive binding protein. Arch Biochem Biophys 413, 236-242.

Tobin, C. (2011). Removal and Replacement of Ribosomal Proteins: Effects on Bacterial Fitness and Ribosome Function (Uppsala: Uppsala Universitet), pp. 93.

Tomoyasu, T., Mogk, A., Langen, H., Goloubinoff, P., and Bukau, B. (2001). Genetic dissection of the roles of chaperones and proteases in protein folding and degradation in the Escherichia coli cytosol. Mol Microbiol 40, 397-413.

Tomsic, J., Vitali, L.A., Daviter, T., Savelsbergh, A., Spurio, R., Striebeck, P., Wintermeyer, W., Rodnina, M.V., and Gualerzi, C.O. (2000). Late events of translation initiation in bacteria: a kinetic analysis. EMBO J 19, 2127-2136.

Tong, Y., Park, I., Hong, B.S., Nedyalkova, L., Tempel, W., and Park, H.W. (2009a). Crystal structure of human elF5A1: insight into functional similarity of human elF5A1 and elF5A2. Proteins 75, 1040-1045.

Tong, Y., Tempel, W., Nedyalkova, L., Mackenzie, F., and Park, H.W. (2009b). Crystal structure of the N-acetylmannosamine kinase domain of GNE. PLoS One 4, e7165.

Tourigny, D.S., Fernandez, I.S., Kelley, A.C., and Ramakrishnan, V. (2013). Elongation factor $\mathrm{G}$ bound to the ribosome in an intermediate state of translocation. Science 340 , 1235490.

Trabuco, L.G., Schreiner, E., Eargle, J., Cornish, P., Ha, T., Luthey-Schulten, Z., and Schulten, K. (2010). The role of L1 stalk-tRNA interaction in the ribosome elongation cycle. J Mol Biol 402, 741-760.

Trobro, S., and Aqvist, J. (2006). Analysis of predictions for the catalytic mechanism of ribosomal peptidyl transfer. Biochemistry 45, 7049-7056.

Ude, S., Lassak, J., Starosta, A.L., Kraxenberger, T., Wilson, D.N., and Jung, K. (2013). Translation elongation factor EF-P alleviates ribosome stalling at polyproline stretches. Science 339, 82-85.

Valeur, B.B.S., M.N. (2012). Excitation Energy Transfer. In Molecular Fluorescence: Principles and Applications (Weinheim: Wiley-VCH), pp. 213-261.

Valle, M., Zavialov, A., Sengupta, J., Rawat, U., Ehrenberg, M., and Frank, J. (2003). Locking and unlocking of ribosomal motions. Cell 114, 123-134.

Van Dyk, T.K., Smulski, D.R., and Chang, Y.Y. (1987). Pleiotropic effects of poxA regulatory mutations of Escherichia coli and Salmonella typhimurium, mutations conferring sulfometuron methyl and alpha-ketobutyrate hypersensitivity. J Bacteriol 169, 4540-4546. 
Victorova, L.S., Kotusov, V.V., Azhaev, A.V., Krayevsky, A.A., Kukhanova, M.K., and Gottikh, B.P. (1976). Synthesis of thioamide bond catalyzed by E. coli ribosomes. FEBS Lett $68,215-$ 218.

Vitagliano, L., Berisio, R., Mastrangelo, A., Mazzarella, L., and Zagari, A. (2001). Preferred proline puckerings in cis and trans peptide groups: implications for collagen stability. Protein Sci 10, 2627-2632.

von der Haar, T. (2008). A quantitative estimation of the global translational activity in logarithmically growing yeast cells. BMC Syst Biol 2, 87.

Voorhees, R.M., Schmeing, T.M., Kelley, A.C., and Ramakrishnan, V. (2010). The mechanism for activation of GTP hydrolysis on the ribosome. Science 330, 835-838.

Wallin, G., and Aqvist, J. (2010). The transition state for peptide bond formation reveals the ribosome as a water trap. Proc Natl Acad Sci U S A 107, 1888-1893.

Wang, S., Corcilius, L., Sharp, P.P., Rajkovic, A., Ibba, M., Parker, B.L., and Payne, R.J. (2017). Synthesis of rhamnosylated arginine glycopeptides and determination of the glycosidic linkage in bacterial elongation factor P. Chem Sci 8, 2296-2302.

Weber, K., Pringle, J.R., and Osborn, M. (1972). Measurement of molecular weights by electrophoresis on SDS-acrylamide gel. Methods Enzymol 26, 3-27.

Weinger, J.S., Parnell, K.M., Dorner, S., Green, R., and Strobel, S.A. (2004). Substrateassisted catalysis of peptide bond formation by the ribosome. Nat Struct Mol Biol 11, 1101-1106.

Weir, B.A., and Yaffe, M.P. (2004). Mmd1p, a novel, conserved protein essential for normal mitochondrial morphology and distribution in the fission yeast Schizosaccharomyces pombe. Mol Biol Cell 15, 1656-1665.

Weixlbaumer, A., Jin, H., Neubauer, C., Voorhees, R.M., Petry, S., Kelley, A.C., and Ramakrishnan, V. (2008). Insights into translational termination from the structure of RF2 bound to the ribosome. Science 322, 953-956.

Witzky, A., Hummels, K.R., Tollerson, R., 2nd, Rajkovic, A., Jones, L.A., Kearns, D.B., and Ibba, M. (2018). EF-P Posttranslational Modification Has Variable Impact on Polyproline Translation in Bacillus subtilis. MBio 9.

Wohlgemuth, I., Brenner, S., Beringer, M., and Rodnina, M.V. (2008). Modulation of the rate of peptidyl transfer on the ribosome by the nature of substrates. J Biol Chem 283, 32229-32235.

Wohlgemuth, I., Pohl, C., Mittelstaet, J., Konevega, A.L., and Rodnina, M.V. (2011). Evolutionary optimization of speed and accuracy of decoding on the ribosome. Philos Trans R Soc Lond B Biol Sci 366, 2979-2986.

Wohlgemuth, I., Pohl, C., and Rodnina, M.V. (2010). Optimization of speed and accuracy of decoding in translation. EMBO J 29, 3701-3709.

Wolff, E.C., Folk, J.E., and Park, M.H. (1997). Enzyme-substrate intermediate formation at lysine 329 of human deoxyhypusine synthase. J Biol Chem 272, 15865-15871.

Wolff, E.C., Kang, K.R., Kim, Y.S., and Park, M.H. (2007). Posttranslational synthesis of hypusine: evolutionary progression and specificity of the hypusine modification. Amino acids 33, 341-350. 
Wolff, E.C., Park, M.H., and Folk, J.E. (1990). Cleavage of spermidine as the first step in deoxyhypusine synthesis. The role of NAD. J Biol Chem 265, 4793-4799.

Wolff, E.C., Wolff, J., and Park, M.H. (2000). Deoxyhypusine synthase generates and uses bound NADH in a transient hydride transfer mechanism. J Biol Chem 275, 9170-9177.

Woolfson, D.N., and Williams, D.H. (1990). The influence of proline residues on alphahelical structure. FEBS Lett 277, 185-188.

Woolstenhulme, C.J., Guydosh, N.R., Green, R., and Buskirk, A.R. (2015). High-precision analysis of translational pausing by ribosome profiling in bacteria lacking EFP. Cell Rep 11, 13-21.

Woolstenhulme, C.J., Parajuli, S., Healey, D.W., Valverde, D.P., Petersen, E.N., Starosta, A.L., Guydosh, N.R., Johnson, W.E., Wilson, D.N., and Buskirk, A.R. (2013). Nascent peptides that block protein synthesis in bacteria. Proc Natl Acad Sci U S A 110, E878-887.

Yamamoto, N., Nakahigashi, K., Nakamichi, T., Yoshino, M., Takai, Y., Touda, Y., Furubayashi, A., Kinjyo, S., Dose, H., Hasegawa, M., et al. (2009). Update on the Keio collection of Escherichia coli single-gene deletion mutants. Mol Syst Biol 5, 335.

Yanagisawa, T., Sumida, T., Ishii, R., Takemoto, C., and Yokoyama, S. (2010). A paralog of lysyl-tRNA synthetase aminoacylates a conserved lysine residue in translation elongation factor P. Nat Struct Mol Biol 17, 1136-1143.

Yanagisawa, T., Takahashi, H., Suzuki, T., Masuda, A., Dohmae, N., and Yokoyama, S. (2016). Neisseria meningitidis Translation Elongation Factor P and Its Active-Site Arginine Residue Are Essential for Cell Viability. PLoS One 11, e0147907.

Yang, Z., and Attygalle, A.B. (2007). LC/MS characterization of undesired products formed during iodoacetamide derivatization of sulfhydryl groups of peptides. J Mass Spectrom 42, 233-243.

Yanofsky, C., and Horn, V. (1994). Role of regulatory features of the trp operon of Escherichia coli in mediating a response to a nutritional shift. J Bacteriol 176, 6245-6254.

Yaron, A., and Naider, F. (1993). Proline-dependent structural and biological properties of peptides and proteins. Crit Rev Biochem Mol Biol 28, 31-81.

Yusupov, M.M., Yusupova, G.Z., Baucom, A., Lieberman, K., Earnest, T.N., Cate, J.H., and Noller, H.F. (2001). Crystal structure of the ribosome at 5.5 A resolution. Science 292, 883896.

Zhang, J., Pan, X., Yan, K., Sun, S., Gao, N., and Sui, S.F. (2015). Mechanisms of ribosome stalling by SecM at multiple elongation steps. Elife 4 .

Zhou, J., Korostelev, A., Lancaster, L., and Noller, H.F. (2012). Crystal structures of $70 \mathrm{~S}$ ribosomes bound to release factors RF1, RF2 and RF3. Curr Opin Struct Biol 22, 733-742.

Zou, S.B., Hersch, S.J., Roy, H., Wiggers, J.B., Leung, A.S., Buranyi, S., Xie, J.L., Dare, K., Ibba, M., and Navarre, W.W. (2012). Loss of elongation factor P disrupts bacterial outer membrane integrity. J Bacteriol 194, 413-425. 


\section{Acknowledgments}

I would like to thank Prof. Marina Rodnina, which gave me the opportunity to pursuit the adventure of a PhD in such an amazing scientific environment. Aside of the scientific competence, I would like to specially thank you, Marina, for giving me the freedom of being active as a student representative for three-quarter of my PhD without having to argue. Your interest in and support of the students matters within the GGNB is highly appreciated by us students and shaped the GGNB to the better.

Here, I would also like to thank Wolfgang not only for scientific advice, but also for advice on where to repair a lovely old Golf and for the conversations regarding, how long a car can run and when it should be replaced.

Coming back to science, I would like to thank my direct supervisors Ingo and Frank, which always gave me scientific advice when requested, while leaving me my desired scientific freedom.

I would also like to acknowledge the members of my my examination board Prof. Kai Tittmann, Prof. Holger Stark, Prof. Ralf Ficner, Prof. Henning Urlaub and Prof. Wolfgang Wintermeyer. Ralf I would like to thank, for his engagement in the GGNB and advice. Henning, thank you for your commitment to the Biomolecules retreat and non-scientific time we spend, cheers.

In addition, I would also like to thank the whole GGNB team for their great work. All GGNB students can consider themselves lucky, having such an amazing supporting team. Kirsten, Steffen and Katrin deserve a special thank here. I appreciate your commitment and also the way how you show your appreciation.

Now it is time to mix science and joy... A big big thanks goes to all the people of the Marina Rodnina Department. Without the help of our brilliant technicians this group would not work as it does. My dear colleagues made the time really enjoyable and I would like to seize the opportunity to thank a few of them, although, I probably will forget more than half of those who would deserve special thanks!

Let's start with my old lab. Riccardo, Anja, Irena, Raffa, Namit and Theo you welcomed me here with open arms and open hearts and I immediately felt home - thank you.

My new lab deserves a special thanks, for being a lot of fun, bearing and supporting me through the toughest phase of my PhD. Ingo, Cris, Raffa, Sandra, Olaf, Prajwal and former members Michi and Albena - thank you! Ingo is not only my supervisor, but also became 
a friend and I enjoyed the time we spend together. Especially the small moments which make people shake their head and musical highlights we shared are appreciated. I wish him and his family all the best for the future! Cris, thank you for answering all stupid questions I came up with and for bringing me the Italian culture closer. Simply having a chat and spending enjoyable non-scientific time with you meant a lot to me. Raffa you won't be singled-out here, but I will pick on you together with the other two R's later. Sandra and Olaf... thank you! Thank you, for everything. Not only scientific help, but also for making this lab to what it is. The casual time is much appreciated and especially in the last phase of my PhD it was much needed! Olaf, thanks for introducing me to some awesome music... you, and sorry for that Sandra, know what I am talking about! Prajwal you are the most inconspicuous member of this lab, but oh my... if you strike, you strike big times! Thank you for making my time here so enjoyable and filled with loud laughers! Special thanks goes to the crew from tower 2, which I got introduced to by a smart, beautiful and lovely incidents (yes, I am bad with names). Fran, associated Martin, Mark, and Felix you added a lot to my life the last $1 \frac{1}{2}$ years here in Göttingen. Highlights are the Stones with J, an oil change with J and of course the recent triathlon!

Thanks also goes to the DS crew - thank you Thomas and Laura for your support and caretaking, putting the PhD from time to time in respect and for having a good time at Willi.

A big big thanks goes to the office 113 of the Stark department! You guys are awesome and my peaceful oasis to overcome the PhD stress. Especially, Lukas as heart of the office and Fabian as comrade in arms and at the glass, deserve a special thank you! Kashish, Karl, Cole and Alex for baring my presence without complaining...

Here I would also like to thank Erik for ProteoPlex assistance and simply good conversations!

Namit, Masha and Sandra I hope our friendship will endure over the PhD and will last as long as we care for it. Thank you for your support and all the good time we spend! I won't spend many words here, you know what I mean. Thank you!

The triple-R team does not only deserve a big thanks for scientific advice, but also for all the time spend together, answering all my Italy related questions and endless discussions on food. Thank you for your support throughout my PhD, you guys mean a lot to me! 
I would also like to thank some old friends Patrick, thanks for the awesome time we shared and all the support you provided. Lars, we know each other for long enough, and as if I would need to explain what our friendship means to me... Even if it is not sufficient to climbing over two garden fences anymore, we stick together!

Speaking of Italy... Elisa, unspeakable thanks goes to you! Some people would say an Avocado number of thanks, or say a PhD is just another crack in the wall... No, but seriously you added a lot too my life. The love you bare for me, your endless support and enduring me through the toughest phase of my $\mathrm{PhD}$ gave me the strength to finish this piece of work! Your presences and even just your existence makes my day a better day. I am looking forward to accept new challenges with you as part of my life!

Lastly, I would like to thank my family which supported me throughout my whole life. The support and understand of my family gave me the strength to pursuit all challenges in my life so far. I would like to point out some of them, and apologise to everyone who went unmentioned: my grandparents, which gave me the undoubted and most natural feeling of security and love, Gudrun and Ralph for support and the right amount of motivation, Katja and the "Jungs" for the time we spend and I promise to be more available, Bärbel and Wolfgang for bring a lot of joy and good times to my life. It goes without saying, my parents. I am deeply grateful for all your advice, criticism, support, love and everything else you invested in Thore and me. Thore, you, Christina and Amelie should be the highlight of the acknowledgment. Throughout my whole life you were there for me, guided me in moments when I had no one to talk to, served as a role model and you set, what I expected of me. Without you, for sure, I would not have achieved what I have achieved so far. In moments of doubt, I could always rely on your factual drive. You and your family showed me what really matters in life and put many things into the right perspectives. I will be there, if wanted or not. That's the beauty of family - you can't choose them. Thank you for everything! 
Alas, four years is far too short a time to work among such excellent and admirable colleagues.

I don't know half of you half as well as I should like, and I like less than half of you half as well as you deserve.

I, uh, I h-have things to do - a lovely girlfriend and a new challenges at Bayer are waiting.

I've put this off for far too long -4 years were more than enough.

I regret to announce - this is The End. I am going now. I bid you all a very fond farewell.

Goodbye - I hope to see you all again and I will miss you! 


\section{Curriculum vitae}

Personal details:

Name

Jan Ole Frister

born 02.02.1989 in Kiel

Address

Immanuel-Kant-Str. 34, 37083 Göttingen

Contacts

Mobile: +49176 63707068

E-mail: ole.frister@gmail.com

jan-ole.frister@mpibpc.mpg.de

\section{Academic studies}

Graduation to Dr. rer. nat. in Physical Biochemistry at the Max Planck Institute for Biophysical Chemistry, Göttingen

$2011-2014$

Master in Microbiology and Biochemistry (M. Sc.) at the Georg-August University of Göttingen

- Thesis: "Involvement of NRPS-like synthases in development and secondary metabolism of Aspergillus nidulans." at the Institute of Microbiology and Genetics

2013

Visiting scientist for NMR-based Structural Biology at the Max Planck Institute for Biophysical Chemistry, Göttingen

- Structural dynamics of alpha-synuclein in the presents of the olgiomerisation inhibiting drug anle138b (US2011293502A1) using CPMG-RD-NMR

$2008-2011$

Bachelor in Biology (B.Sc.) at the Leibniz University of Hannover

- Thesis: "Analysis of the heterologous expression of the Ansamitocin-amidsynthase (Asm9) from Actinosynnema pretiosum and large scale mutabiosynthesis of $\mathrm{N}$-desmethylansamitocin." at the Institute of Organic Chemistry

$2005-2008 \quad$ Wilhelm-Busch-Gymnasium in Stadthagen, Abitur

\section{International experiences}

$2012-2013$

Research stay at the University of Cambridge, Department of Biochemistry, Cambridge, UK

- Heterologous production of the natural product nigericin

\section{Leadership and social activities}

$2014-2017$

Student representative of the "Göttingen graduate school of Neurosciences, Biophysics, and Molecular Biosciences" (GGNB)

- Negotiate and represent the students interest in front of the 
GGNB board, the president of the University and during the visit of the president Federal Republic of Germany

$2015-2016$

PhD student representative of the "Georg-August University School of Science" (GAUSS)

- Representation of the students interests at the GAUSS board

- Representative of more than $1.000 \mathrm{PhD}$ students in natural science

Honors

2014

Max Planck Doctoral stipend

2014

Cambridge European Scholarships

2014

Fellowship at Pembroke College at the University of Cambridge, UK

\section{Addition qualification}

- Participant at the $12^{\text {th }}$ Intercultural communication workshop:

"Working across borders, communicating across cultures" by Petersen \& Petersen

- Seminar on "Medical chemistry" by Abbott Laboratories

- Course on "Viral transfection systems" including S2 work at German Primate Center

- Seminar on "Project management for young scientists" by Golin Managment

- Course on "Drug Discovery and Project Management in the Pharmaceutical Industry" by Dr. Willmar Schwabe Arzneimittel GmbH \& Co. KGa

- Certificate on Good Manufacturing Practice by Sartorius, Juno Therapeutics and other companies

\section{Personal interests}

Road biking, martial arts, travelling and cooking 

For what is a man, what has he got If not himself, then he has naught

To say the things he truly feels And not the words of one who kneels

The record shows I took the blows And did it my way Yes, it was my way

Frank Sinatra, I did it my way 

